\title{
FROM
}

\section{PHOTON PATHS}

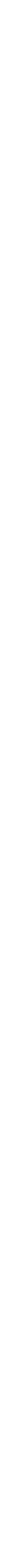




\section{From photon paths to pollution plumes: \\ better radiative transfer calculations to monitor $\mathrm{NO}_{x}$ emissions with OMI and TROPOMI}

Alba Lorente Delgado 


\section{Thesis committee}

\section{Promotor}

Prof. Dr M. C. Krol

Professor of Air Quality and Atmospheric Chemistry,

Meteorology and Air Quality Group, Wageningen University \& Research

\section{Co-promotor}

Dr K. F. Boersma

Research Scientist, Meteorology and Air Quality Group

Wageningen University \& Research

\section{Other members:}

Dr M. de Maziére, BIRA-IASB Royal Belgian Institute for Space Aeronomy, Brussels, Belgium

Dr O. P. Hasekamp, SRON Netherlands Institute for Space Research, Utrecht, the Netherlands Prof. Dr G. Velders, Utrecht University, the Netherlands

Prof. Dr M. Herold, Wageningen University \& Research

This research was conducted under the auspices of the Socio-Economicand Natural Sciences of the Environment (SENSE) Graduate School 


\title{
From photon paths to pollution plumes: better radiative transfer calculations to monitor $\mathrm{NO}_{x}$ emissions with OMI and TROPOMI
}

\author{
Alba Lorente Delgado
}

Thesis

submitted in fulfilment of the requirements for the degree of doctor at

Wageningen University

by the authority of the Rector Magnificus

Prof. Dr A.P.J. Mol,

in the presence of the

Thesis Committee appointed by the Academic Board

to be defended in public

on Thursday 23 May 2019

at 11 a.m. in the Aula. 
A. Lorente Delgado

From photon paths to pollution plumes: better radiative transfer calculations to monitor $\mathrm{NO}_{x}$ emissions with OMI and TROPOMI 208 pages.

PhD thesis, Wageningen University \& Research, Wageningen, The Netherlands (2019) With references, with summary in English

DOI: https://doi.org/10.18174/474563

ISBN: 978-94-6343-932-9 
"The big artist keeps a sharp eye on Nature [...] learns what she does with light (the biggest tool), then the colour and then the form, and appropriates the knowledge to his own use."

Thomas Eakins (1867), American realist painter 



\section{Contents}

Page

$\begin{array}{lll}\text { Contents } & \text { vi }\end{array}$

1 Introduction $\quad 1$

1.1 Trace gases in the atmosphere . . . . . . . . . . . . . . . 2

1.2 Satelite remote sensing of atmospheric composition in the UV-Vis . . . . . 11

1.3 Radiative transfer in the atmosphere . . . . . . . . . . . . . . 17

1.4 Thesis outline . . . . . . . . . . . . . . . . . 20

2 QA4ECV $\mathrm{NO}_{2}$ retrieval algorithm: on the way to TROPOMI 23

2.1 QA4ECV for $\mathrm{NO}_{2} \ldots \ldots \ldots \ldots \ldots . \ldots \ldots$

$2.2 \mathrm{NO}_{2}$ QA4ECV retrieval algorithm . . . . . . . . . . 26

2.2.1 Slant column density . . . . . . . . . . . . . . . . . . . . . . . . . . . . . . . . . . . 28

2.2.2 Stratospheric $\mathrm{NO}_{2}$ column estimation . . . . . . . . . . . . . . 31

2.2.3 Air mass factor calculation . . . . . . . . . . . . . . . 33

$2.3 \mathrm{NO}_{2}$ QA4ECV climate data record . . . . . . . . . . . . . . . 41

2.4 QA4ECV $\mathrm{NO}_{2}$ product validation . . . . . . . . . . . . . . . . . . . 43

2.4.1 Comparison to DOMINO . . . . . . . . . . . . . . 43

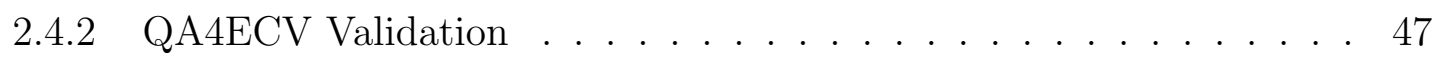

2.5 Conclusions . . . . . . . . . . . . . . . . . . 50

3 Structural uncertainty in air mass factor calculation for $\mathrm{NO}_{2}$ and $\mathrm{HCHO}$ satellite retrievals $\quad 53$

3.1 Introduction . . . . . . . . . . . . . . . . . . . . . 55

3.2 Methods . . . . . . . . . . . . . . . . . . 57

3.2.1 AMF calculation process . . . . . . . . . . . . . 57

3.2 .2 Participating models . . . . . . . . . . . . . . 61

3.3 Results . . . . . . . . . . . . . . . . . . . . . 64

3.3 .1 TOA reflectances . . . . . . . . . . . . . . . 64

3.3.2 $\quad \mathrm{NO}_{2}$ and $\mathrm{HCHO}$ altitude-dependent (box-) air mass factors . . . . . 67 
3.3.3 Tropospheric air mass factors . . . . . . . . . . . . . . . . . 70

Harmonized settings . . . . . . . . . . . . . . . . . . . . . . . . . . . . 70

Cloud correction: IPA vs. clear-sky AMF . . . . . . . . . . . 73

Round robin comparison . . . . . . . . . . . . . . . . . . . . . . . . . . . . . .

3.3.4 Stratospheric air mass factors . . . . . . . . . . . . . . . 84

3.4 Conclusions . . . . . . . . . . . . . . . . . . 86

4 The importance of surface reflectance anisotropy for cloud and $\mathrm{NO}_{2}$ retrievals from GOME-2 and OMI $\quad 89$

4.1 Introduction . . . . . . . . . . . . . . . . . . . . . . . 91

4.2 Evidence of the influence of surface reflectance anisotropy on LER climatologies and cloud retrievals . . . . . . . . . . . . . . . . 93

4.3 Reflectance simulations with surface BRDF in DAK . . . . . . . . . . 98

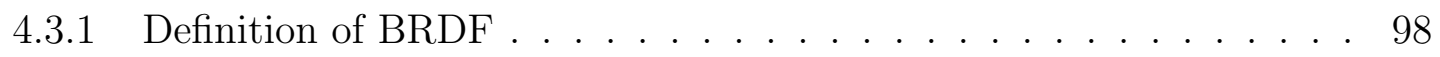

4.3.2 Surface BRDF implementation in DAK . . . . . . . . . . . . 100

4.3.3 Evaluation of surface BRDF effects in DAK TOA reflectances . . . 103

4.4 Role of surface BRDF in cloud retrievals . . . . . . . . . . . . . . . 106

4.4.1 Synthetic cloud fraction retrieval . . . . . . . . . . . . 106

4.4 GOME-2A cloud fraction simulations . . . . . . . . . . . . . 109

4.5 Role of surface BRDF in $\mathrm{NO}_{2}$ retrievals . . . . . . . . . . . . . . . . . 109

4.5.1 BRDF effects on tropospheric $\mathrm{NO}_{2}$ air mass factors . . . . . . . . 112

4.5.2 GOME-2A tropospheric $\mathrm{NO}_{2}$ air mass factors . . . . . . . . . . . 114

4.6 Discussion and conclusions . . . . . . . . . . . . . . . . 117

5 Quantification of nitrogen oxides emissions from build-up of pollution $\begin{array}{lr}\text { over Paris with TROPOMI } & 121\end{array}$

5.1 Introduction . . . . . . . . . . . . . . . . . . . . . . . 122

5.2 Results . . . . . . . . . . . . . . . . . . . . . . . 123

5.2.1 $\mathrm{NO}_{2}$ build-up in air advected over Paris . . . . . . . . . . . . 123

5.2 .2 Interpreting line densities . . . . . . . . . . . . . . . . 125

5.2.3 Estimating $\mathrm{NO}_{x}$ emissions from TROPOMI line densities . . . . . . 125

5.2.4 Day-to-day and spatial variability in $\mathrm{NO}_{x}$ emissions . . . . . . . . . 129

5.3 Discussion . . . . . . . . . . . . . . . . . . . . . . 130

5.4 Methods . . . . . . . . . . . . . . . . . . . . . 131

5.4 .1 TROPOMI sensor . . . . . . . . . . . . . . . . . . 131

$5.4 .2 \mathrm{NO}_{2}$ retrieval algorithm . . . . . . . . . . . . . . . 131

5.4 .3 TROPOMI $\mathrm{NO}_{2}$ data filtering . . . . . . . . . . . . . . . . 132

5.4 Line densities . . . . . . . . . . . . . . . . . . . . . . . 133

5.4.5 Boundary layer information on wind and chemistry . . . . . . . . 134

5.4.6 Simulating line densities with a superposition model . . . . . . . . . 134 
5.4.7 Daily $\mathrm{NO}_{x}$ emissions over Paris from the TNO-MACC-III inventory 135

6 General discussion and outlook

6.1 Radiative transfer and air mass factor calculation . . . . . . . . . . . . 137

6.2 Surface reflectance anisotropy . . . . . . . . . . . . . . . . . . . . . 140

6.3 TROPOMI $\mathrm{NO}_{2}$ measurements for daily $\mathrm{NO}_{x}$ emission estimates . . . . . . 142

Summary

Supplementary material

References

Acknowledgements

Graduate School Certificate 



\section{Introduction}

Nitrogen dioxide $\left(\mathrm{NO}_{2}\right)$ is a highly important air pollutant that can be monitored from space. Figure 1.1 is an example of global $\mathrm{NO}_{2}$ measurements made during 2005 by the Ozone Monitoring Instrument $(\mathrm{OMI})^{1} . \mathrm{NO}_{2}$ in the atmosphere has a clear signature in the light measured by the satellite, which makes it an ideal candidate to be monitored from space. However, $\mathrm{NO}_{2}$ measured from space is an indirect measurement with multiple assumptions and error sources, so one of the main goals of this thesis is to improve $\mathrm{NO}_{2}$ satellite measurements and the errors associated with them (Chapter 2, 3 and 4). $\mathrm{NO}_{2}$ has a relatively short lifetime (i.e. it does not stay in the atmosphere for more than 2-12 hours), so fresh emissions have a strong imprint in the atmosphere and appear clearly in the satellite measurements. This is key for using satellite measurements to estimate the underlying emissions, as we do in Chapter 5 to quantify the emissions from urban areas like Paris.

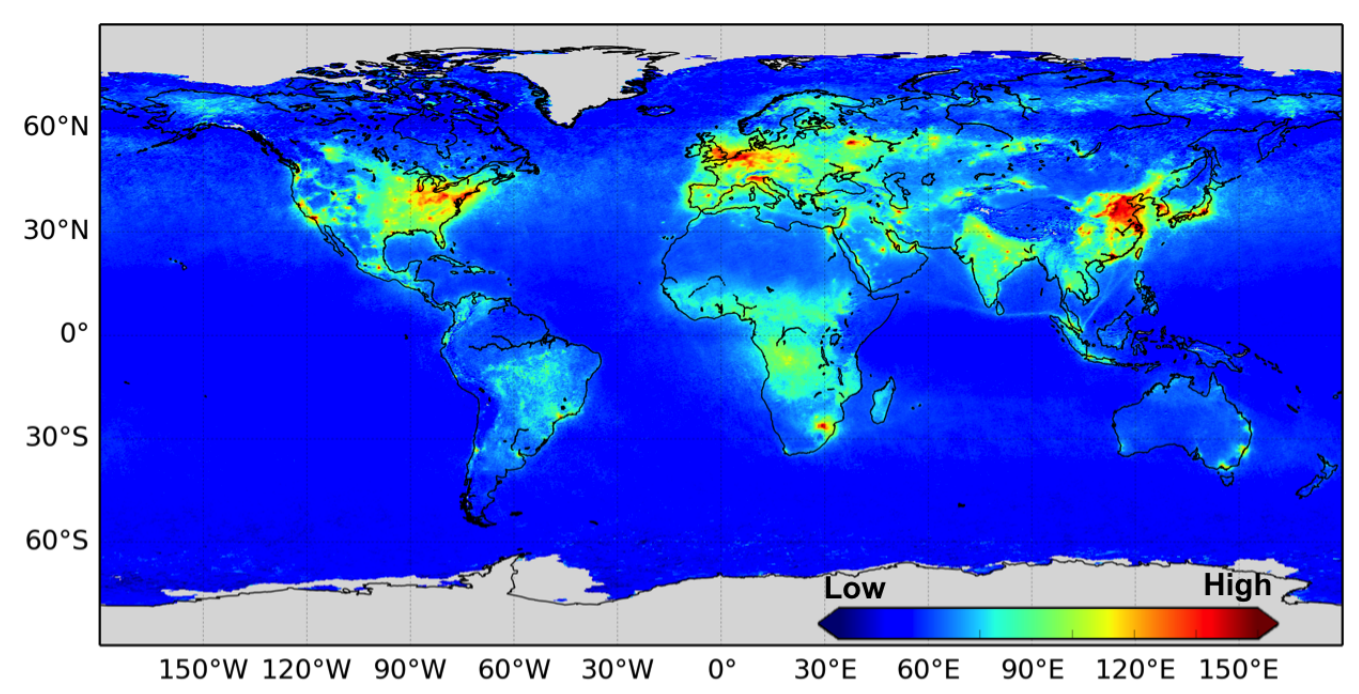

Figure 1.1: Mean $\mathrm{NO}_{2}$ amount in 2005 measured from OMI averaged in a $0.04^{\circ} \times 0.08^{\circ}$ latitude - longitude grid.

\footnotetext{
${ }^{1}$ Satellite instruments like OMI measure $\mathrm{NO}_{2}$ vertical column density (in molecules per $\mathrm{cm}^{2}$ ), which is the amount of $\mathrm{NO}_{2}$ integrated vertically along the atmosphere.
} 
In this first chapter we introduce the composition of the atmosphere and discuss the relevance of $\mathrm{NO}_{2}$ (and other atmospheric constituents) for Earth's climate system and for global and local air pollution and its harmful health effects. We also highlight the importance of monitoring atmospheric composition from space and its various applications. Finally, we give a brief introduction on the remote sensing measurement principles (Sect. 1.2) and radiative transfer in the atmosphere (Sect. 1.3).

\subsection{Trace gases in the atmosphere}

Trace gases are the gases that are found in very small concentrations in the atmosphere, which typically constitute less than $1 \%$ of the Earth's total atmospheric composition. Some of these gases are carbon dioxide $\left(\mathrm{CO}_{2}\right)$, methane $\left(\mathrm{CH}_{4}\right)$, ozone $\left(\mathrm{O}_{3}\right)$, nitrogen oxides $\left(\mathrm{NO}_{x}=\mathrm{NO}+\mathrm{NO}_{2}\right)$ and volatile organic compounds (VOCs). Despite the small concentration of these trace gases, they play an important role in the Earth's climate because among them the main agents of Earth's radiative forcing (i.e. the perturbation of the Earth's energy budget) are found. The different components of the radiative forcing based on emitted gases, aerosols and precursors are shown in Figure 1.2. Short-life species like $\mathrm{NO}_{x}$ and VOCs are essential in the atmospheric photochemical processes that affect the main forcing agents (Myhre et al., 2013). Ozone and aerosols are defined as Essential Climate Variables (ECV) by the World Meteorological Organization (WMO) Global Climate Observing System (GCOS), and in $2011 \mathrm{NO}_{2}$ and formaldehyde (HCHO, a main VOC oxidation product) were included as precursors of these ECVs (together with sulfur dioxide $\left(\mathrm{SO}_{2}\right)$ and carbon monoxide $(\mathrm{CO})$ ). They were included in the ECV framework because they contribute to the characterisation of Earth's past, present and future climate and they can be monitored from existing observation systems (Bojinski et al., 2014).

Short lived gases like $\mathrm{NO}_{x}$ and non-methane (NM) VOCs contribute to the radiative forcing because they affect the production and losses of the main forcing agents $\mathrm{CO}_{2}$, $\mathrm{CH}_{4}$ and $\mathrm{O}_{3}$ (Myhre et al., 2013). This results in NMVOCs having an overall positive (i.e. warming) effect in the radiative forcing. $\mathrm{NO}_{x}$ is key in $\mathrm{O}_{3}$ production, therefore $\mathrm{NO}_{x}$ emissions also result in positive radiative forcing. Apart from this, $\mathrm{NO}_{x}$ is key in the production of the hydroxyl radical $\mathrm{OH}$, which is the main oxidant in the troposphere and the main sink of $\mathrm{CH}_{4}$. Therefore, $\mathrm{NO}_{x}$ indirectly reduce the levels of $\mathrm{CH}_{4}$ and leads to a negative radiative forcing (i.e. cooling) (Shindell et al., 2009) (see Fig. 1.2). $\mathrm{NO}_{x}$ have also an indirect effect on radiative forcing because it influences the formation of secondary aerosols, which scatter part of the solar radiation back to space, thus contributing further to the negative forcing. Solomon et al. (1999) showed that $\mathrm{NO}_{2}$ can also affect local instantaneous radiative forcing by absorbing solar radiation under polluted conditions and 
when $\mathrm{NO}_{2}$ is produced by lightning in electrically active storms. All the non-linearities in the physical and chemical processes in which $\mathrm{NO}_{x}$ is involved make it very difficult to estimate the net $\mathrm{NO}_{x}$ radiative forcing. The latest IPCC report estimated that the net effect due to anthropogenic $\mathrm{NO}_{x}$ emissions is a negative radiative forcing of $-0.15 \mathrm{~W} / \mathrm{m}^{2}$ (Myhre et al., 2013).

$\mathrm{CO}_{2}$ and $\mathrm{CH}_{4}$ are the main contributors to climate radiative forcing, and their positive forcing of about $2.5 \mathrm{~W} / \mathrm{m}^{2}$ results in an enhanced greenhouse effect. $\mathrm{O}_{3}$ is photochemically produced in the atmosphere (i.e. not directly emitted) and it constitutes a relatively shortlived gas compared to $\mathrm{CO}_{2}$ or $\mathrm{CH}_{4}$. The radiative forcing for tropospheric $\mathrm{O}_{3}$ (about $\left.0.5 \mathrm{~W} / \mathrm{m}^{2}\right)$ is attributed to anthropogenic emissions of $\mathrm{CH}_{4}, \mathrm{NO}_{x}, \mathrm{CO}$ and NMVOCs. The radiative forcing of ozone in the stratosphere $\left(-0.15 \mathrm{~W} / \mathrm{m}^{2}\right)$ is attributed to ozone depletion by halocarbons (see Fig. 1.2). For $\mathrm{O}_{3}$, there is a strong regional variability on how its precursor emissions contribute to its radiative forcing. Naik et al. (2005) found that the $\mathrm{O}_{3}$ radiative forcing is more sensitive to changes in precursor emissions over East and Southeast Asia. This is because in these tropical regions, $\mathrm{O}_{3}$ and its precursors are transported to the upper troposphere, where $\mathrm{O}_{3}$ production efficiency is higher. Tropical regions are characterized by high photochemical activity, which together with high VOCs emissions from natural vegetation and biomass burning leads to a more efficient $\mathrm{O}_{3}$ production compared to mid-latitude regions (Naik et al., 2005).

Trace gases are not only relevant for radiative forcing and climate, they also affect the quality of the air that we breathe, which impacts human health and ecosystems. The European Union sets limits to concentrations of several pollutants that should not be exceeded to guarantee a good air quality. Among these pollutants are fine and coarse particles (i.e. particulate matter (PM) with diameters smaller than $2.5 \mu \mathrm{m}$ and $10 \mu \mathrm{m}$, respectively), $\mathrm{SO}_{2}, \mathrm{NO}_{2}, \mathrm{CO}, \mathrm{O}_{3}$, benzene and several toxic metals. For $\mathrm{PM}$ and $\mathrm{O}_{3}$ the EU limits are less strict than the World Health Organization (WHO) air quality guidelines (see Table 1.1), which are set to protect human health. The last "Air Quality in Europe" report by the EEA estimated that in 2016, $8 \%$ of the EU urban population was exposed to harmful levels of $\mathrm{NO}_{2}, 8 \%$ and $19 \%$ was exposed to harmful $\mathrm{PM}_{10}$ and $\mathrm{PM}_{2.5}$ levels and $30 \%$ to $\mathrm{O}_{3}$ levels above the limits. These percentages increase to $85 \%, 52 \%$ and $98 \%$ respectively if the WHO guidelines are considered (EEA, 2018) and these statistics applied globally result in $91 \%$ of the world's population living in places with poor air quality (WHO, 2016). These numbers show that severe measures are still required to clean up the air further and show the relevance of monitoring the pollutants at high temporal and spatial resolution to control where and when the highest concentrations occur. This allows to assess the effectiveness of the control strategies to improve air quality, and $\mathrm{NO}_{2}$ satellite measurements offer a unique perspective for this purpose due to the possibility of having daily global coverage at high spatial resolution, as we show in 


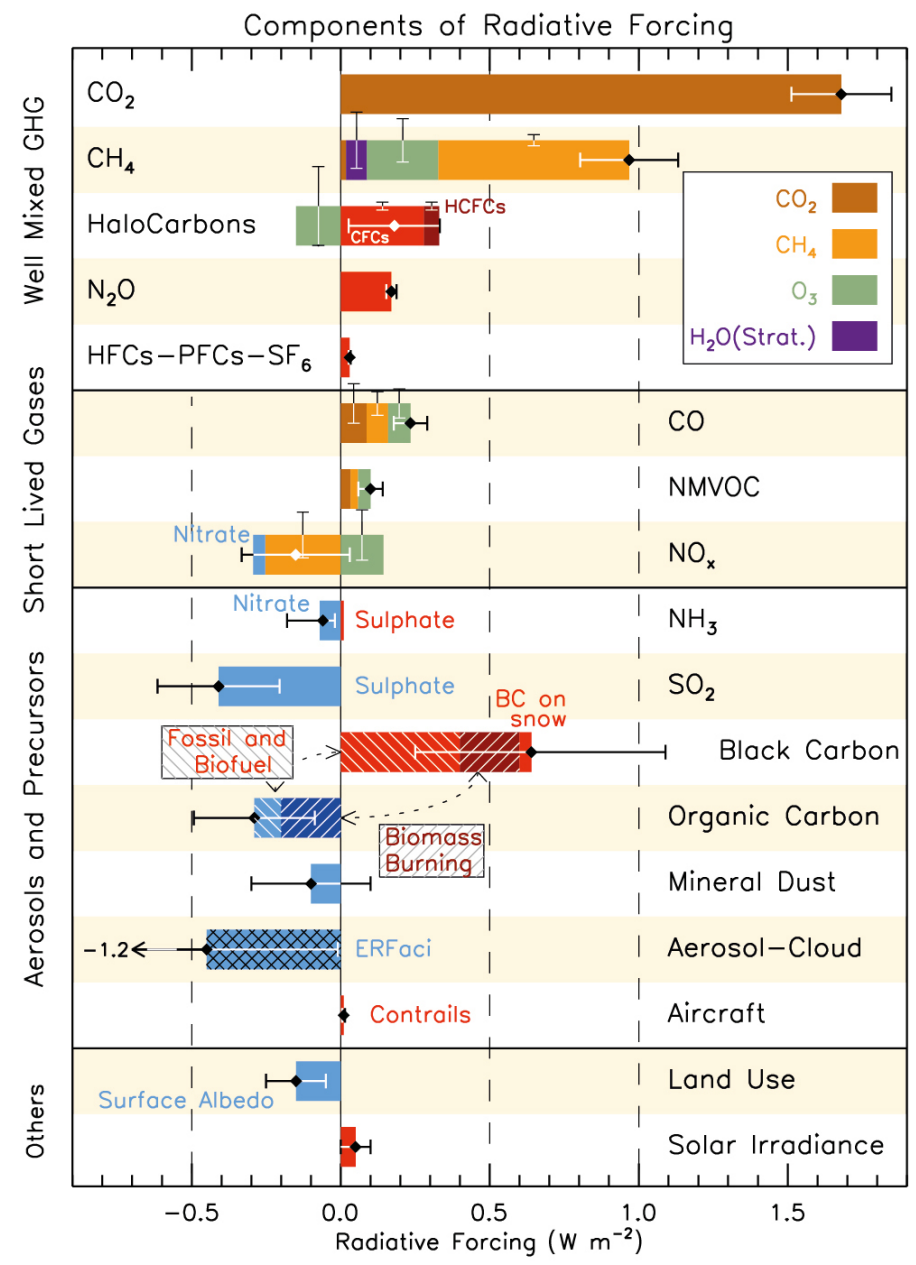

Figure 1.2: Radiative forcing for the period 1750-2011 based on emitted compounds. Different colors in the emitted components represent the different forcing agents they affect. The vertical bars indicate the relative uncertainty of the radiative forcing induced by each component (from Myhre et al. (2013)).

some examples later in this section.

Table 1.1: European Union air quality standards and WHO air quality guideline values for $\mathrm{PM}_{10}, \mathrm{PM}_{2.5}, \mathrm{NO}_{2}$ (annual mean) and $\mathrm{O}_{3}$ (8 hour mean) in $\mu \mathrm{g} / \mathrm{m}^{3}$.

\begin{tabular}{ccccc}
\hline & $\mathbf{P M}_{10}$ & $\mathbf{P M}_{10}$ & $\mathbf{N O}_{2}$ & $\mathbf{O}_{3}$ \\
\hline EU & 25 & 40 & 40 & 120 \\
\hline WHO & 10 & 20 & 40 & 100 \\
\hline
\end{tabular}

The most recent WHO air pollution report (WHO, 2016) estimated that the exposure to air pollution causes around 4 million premature deaths worldwide mainly due to cardiovascular diseases (heart diseases and strokes), respiratory infections and lung diseases including cancer. Ambient fine particles are consistently associated with mortality, hence many studies assess air pollution exposure from the concentrations of particulate 
matter (e.g. WHO (2016), Hales et al. (2012)). Because harmful levels of PM and $\mathrm{NO}_{2}$ are frequently correlated, the particular attribution of these pollutants is not straightforward and adjustments need to be made to quantify their health effects separately (WHO, 2013). The DUELS study (Fischer et al., 2015) analysed air pollution effects in a 7 million population from The Netherlands. They showed that long-term exposure to $\mathrm{PM}_{10}$ after adjustment for $\mathrm{NO}_{2}$ remained associated to non-accidental mortality, mortality from respiratory diseases, lung cancer mortality and cardiovascular mortality. For $\mathrm{NO}_{2}$, they found associations to all mortalities but not to cardiovascular mortality, and after adjustment for $\mathrm{PM}_{10}$ associations remained for non-accidental mortality and lung cancer mortality. This study shows that both $\mathrm{NO}_{2}$ and PM should be taken into account when analysing the effects of long-term exposure to air pollution. The differences before and after adjustments show that these two pollutants characterize air pollution and its health effects differently, but also that they are both causing health damage.

Epidemiological studies mostly use models and in-situ observations to estimate the population exposure to air pollution. In several studies satellite measurements of $\mathrm{NO}_{2}$ have been proposed as a reliable tool to measure the spatial variability and long term exposure of $\mathrm{NO}_{2}$ (e.g. Geddes et al., 2016) and they have been used to evaluate health impacts (e.g. Anenberg et al., 2018). The use of satellites improves exposure assessment (e.g. Hoek et al., 2015) and can complement the use of models in epidemiological studies. In order to bring forward the use of satellites for these type of applications, we need to have reliable satellite measurements and uncertainty estimates, and an active collaboration between satellite and 'Public Health' experts is indispensable.

\section{Tropospheric $\mathrm{NO}_{x}-\mathrm{VOC}-\mathrm{O}_{3}$ chemistry}

$\mathrm{O}_{3}$ is a secondary pollutant that is formed in the troposphere in the presence of light and other chemical components such as VOCs and $\mathrm{NO}_{x}$. Globally, the most abundant VOCs are isoprene and monoterpenes, mostly emitted by natural sources in the tropical regions (Sindelarova et al., 2014). Anthropogenic sources of VOCs account for $15 \%$ of the total VOCs emissions, and most of these $15 \%$ occur in agriculture, transport (mainly emission of hydrocarbons) and industrial processes and product use. McDonald et al. (2018) showed that chemical products constitute half of the VOC emissions in industrialized U.S. cities. This contribution has increased relative to the contribution from transportation, sector for which emissions have effectively been controlled in the last decades. Globally, anthropogenic emissions are the largest source of annual $\mathrm{NO}_{x}$ emissions, although natural emissions (mainly biomass burning, lightning and soils) can substantially contribute to the total $\mathrm{NO}_{x}$ emissions budget. In industrialized urban areas, anthropogenic $\mathrm{NO}_{x}$ emissions correspond to around $90 \%$ of the total $\mathrm{NO}_{x}$ emissions. They are mainly emitted in combustion processes, so most of the emissions are from the transport sector. Figure 1.3 
shows the fraction of anthropogenic $\mathrm{NO}_{x}$ and non-methane VOC emissions by different sectors in the European Union for the year 2016.

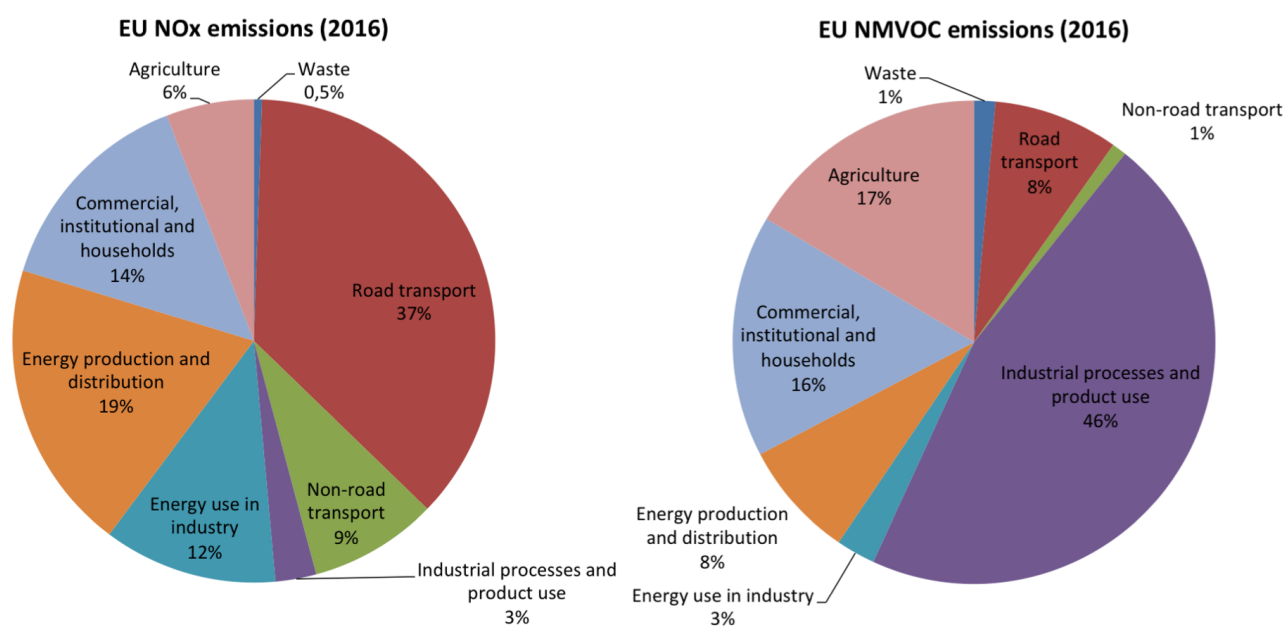

Figure 1.3: Fraction of anthropogenic nitrogen oxides $\left(\mathrm{NO}_{x}\right)$ and non-methane volatile organic compounds (NMVOC) emitted by different sectors in the European Union in 2016. Reported total emissions in 2016 were $8.5 \mathrm{Tg} \mathrm{NO}_{x}$ and $8 \mathrm{Tg}$ NMVOCs. Source: EU emission inventory report 1990-2016 under the UNECE Convention on Long-range Trans-boundary Air Pollution (LRTAP).

Most of the $\mathrm{NO}_{x}$ are emitted in the form of $\mathrm{NO}$, and then a rapid cycling occurs between $\mathrm{NO}$ and $\mathrm{NO}_{2}$, which is called the photostationary state:

$$
\begin{aligned}
& \mathrm{NO}+\mathrm{O}_{3} \rightarrow \mathrm{NO}_{2}+\mathrm{O}_{2} \\
& \mathrm{NO}_{2}+h v \stackrel{\mathrm{O}_{2}}{\longrightarrow} \mathrm{NO}+\mathrm{O}_{3}
\end{aligned}
$$

At night, most of the $\mathrm{NO}_{x}$ is present as $\mathrm{NO}_{2}$ due to the lack of light to photolyse $\mathrm{NO}_{2}$ via reaction $\mathrm{R} 2$. The principal sink of $\mathrm{NO}_{x}$ is the oxidation to $\mathrm{HNO}_{3}$ by $\mathrm{OH}$ during daytime. At night $\mathrm{NO}_{x}$ is oxidized by $\mathrm{O}_{3}$, and will react through $\mathrm{N}_{2} \mathrm{O}_{5}$ on aerosol surfaces. The photostationary state (R1-R2) is a null cycle in which $\mathrm{O}_{3}$ is continuously being produced and consumed. For $\mathrm{O}_{3}$ to accumulate in the troposphere, an additional pathway is required to convert $\mathrm{NO}$ to $\mathrm{NO}_{2}$ without destroying $\mathrm{O}_{3}$. This occurs in the photochemical oxidation of VOCs via reactions R4 and R6 (Jacob, 1999):

$$
\begin{gathered}
\mathrm{RH}+\mathrm{OH} \stackrel{\mathrm{O}_{2}}{\rightarrow} \mathrm{RO}_{2}+\mathrm{H}_{2} \mathrm{O} \\
\mathrm{RO}_{2}+\mathrm{NO} \rightarrow \mathrm{RO}+\mathrm{NO}_{2} \\
\mathrm{RO}+\mathrm{O}_{2} \rightarrow \mathrm{R}^{\prime} \mathrm{CHO}+\mathrm{HO}_{2}
\end{gathered}
$$




$$
\mathrm{HO}_{2}+\mathrm{NO} \rightarrow \mathrm{OH}+\mathrm{NO}_{2}
$$

The net reaction is then:

$$
\mathrm{RH}+4 \mathrm{O}_{2} \rightarrow \mathrm{R}^{\prime} \mathrm{CHO}+2 \mathrm{O}_{3}+\mathrm{H}_{2} \mathrm{O}
$$

In this chain, RH refers to any hydrocarbon. From reactions $\mathrm{R} 4$ and $\mathrm{R} 6, \mathrm{NO}_{2}$ photolyses and creates $\mathrm{O}_{3}$. This chain of reactions is broken by loss of $\mathrm{HO}_{x}$ radicals $\left(\mathrm{HO}_{x}=\mathrm{OH}\right.$ $\left.+\mathrm{HO}_{2}\right)$ in two different paths, depending on the relative concentrations of hydrocarbons and $\mathrm{NO}_{x}$. Thus the net production of ozone within this chain depends on the availability of hydrocarbons and $\mathrm{NO}_{x}$.

The atmosphere will be hydrocarbon limited if there are not enough hydrocarbons that oxidize to form $\mathrm{RO}_{2}$ via reaction $\mathrm{R} 3$. In this situation, the amount of $\mathrm{NO}_{2}$ present in the atmosphere will be high compared to the concentration of hydrocarbons, $\mathrm{so}^{\mathrm{NO}_{2}}$ will deplete $\mathrm{OH}$ by oxidation to $\mathrm{HNO}_{3}$. In contrast, the atmosphere will be $\mathrm{NO}_{x}$ limited if there are not enough $\mathrm{NO}$ to convert $\mathrm{HO}_{2}$ to $\mathrm{OH}$ via $\mathrm{R} 6$, so $\mathrm{HO}_{2}$ gets lost to $\mathrm{H}_{2} \mathrm{O}_{2}$ and the chain ends. Figure 1.4 shows simulated ozone concentrations as a function of $\mathrm{NO}_{x}$ and hydrocarbon emissions. In the hydrocarbon limited regime, $\mathrm{O}_{3}$ concentrations increase with hydrocarbon emissions and decrease slightly with $\mathrm{NO}_{x}$ emissions. In the $\mathrm{NO}_{x}$ limited regime, where hydrocarbon emissions are higher, there are enough hydrocarbons for $\mathrm{NO}_{x}$ to create $\mathrm{O}_{3}$, so an increase in $\mathrm{NO}_{x}$ result in an increase of $\mathrm{O}_{3}$ concentrations.

The production of ozone depends strongly and in a non-linear way on the relative concentrations of hydrocarbons and nitrogen oxides in the troposphere. A study in the city of Madrid, Saiz-Lopez et al. (2017) showed that despite the decrease in $\mathrm{NO}_{2}$ concentrations in the period 2007-2014, $\mathrm{O}_{3}$ concentrations increased, which might be a consequence of the city of Madrid being "hydrocarbon limited". Orographical and meteorological conditions also play an important role in $\mathrm{O}_{3}$ pollution episodes in megacities like Madrid (Querol et al., 2018), which makes the interpretation of urban boundary layer chemistry even more complex. The regime under which ozone is formed has a seasonal dependency, and when the transition from hydrocarbon to $\mathrm{NO}_{x}$ limited happens is crucial for the effectiveness of mitigation strategies (Jacob et al., 1995). Because it is highly relevant to have knowledge on the prevalent chemical regime, the monitoring of $\mathrm{NO}_{2}$ and VOCs not only from the ground but also from space is necessary to tackle tropospheric and surface ozone pollution problems efficiently.

The study by Jin et al. (2017) used 10 years of satellite measurements to study trends of the $\mathrm{O}_{3}$ formation regime: using the ratio of $\mathrm{OMI} \mathrm{NO}_{2}$ measurements (as an indicator for $\mathrm{NO}_{x}$ ) and formaldehyde (as an indicator for VOCs), they classified different regions around the world as $\mathrm{NO}_{x}$ or hydrocarbon limited. Figure 1.5 shows that European and U.S. cities 


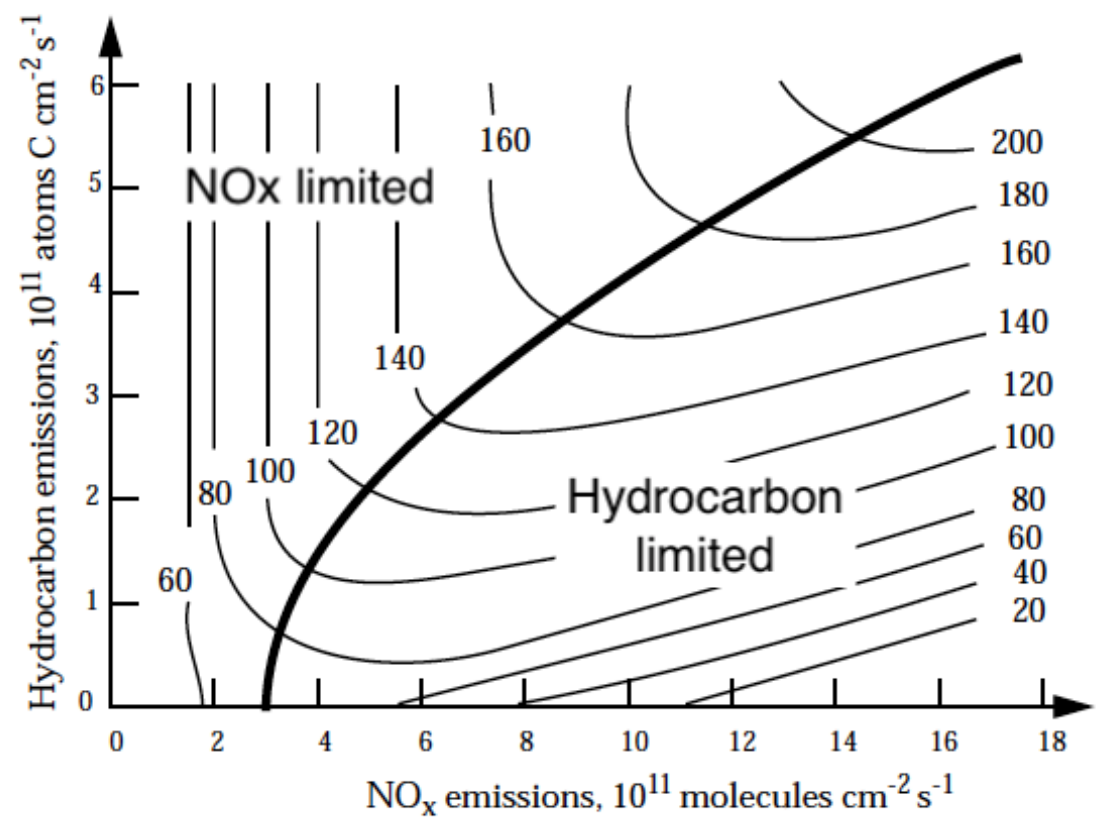

Figure 1.4: Contour lines of ozone mixing ratio (in ppb) as a function of $\mathrm{NO}_{x}$ and hydrocarbon emissions. Thick black line separates $\mathrm{NO}_{x}$ limited and hydrocarbon limited ozone production regimes (adapted from Jacob (1999)).

are becoming $\mathrm{NO}_{x}$ limited due to the decrease in $\mathrm{NO}_{x}$ emissions. Because of this change in the $\mathrm{O}_{3}$ production regime, in those cities $\mathrm{NO}_{x}$ emission controls might be now more effective than a decade ago. The use of satellite measurements results in a global overview of $\mathrm{O}_{3}$ formation regimes that is not feasible with other type of measurements. However, it is conditioned by the uncertainties linked to satellite retrievals, which highlights the need to characterize and reduce the uncertainties, one of the main motivations of this thesis.

\section{Atmospheric composition from space}

Satellite measurements have been widely used to monitor $\mathrm{O}_{3}$ (e.g. $\mathrm{Fu}$ et al. (2018)) and its main precursors $\mathrm{NO}_{x}$ and VOCs. Formaldehyde (HCHO) is a major product in the oxidation of most VOCs, therefore satellite measurements of $\mathrm{HCHO}$ can give unique information on the spatial and temporal patterns of the main VOC sources (e.g. Zhu et al. (2017), Bauwens et al. (2016)). $\mathrm{NO}_{2}$ satellite measurements can be used to estimate $\mathrm{NO}_{x}$ emissions from different sources, including cities (e.g. Beirle et al. (2016)), ships (e.g. Boersma et al. (2015)), lightning (e.g. Boersma et al. (2005)) and fires (e.g. Castellanos et al. (2014), Mebust et al. (2011)). Because satellites have been measuring atmospheric composition for more than two decades, the evolution in time of emissions can also be 


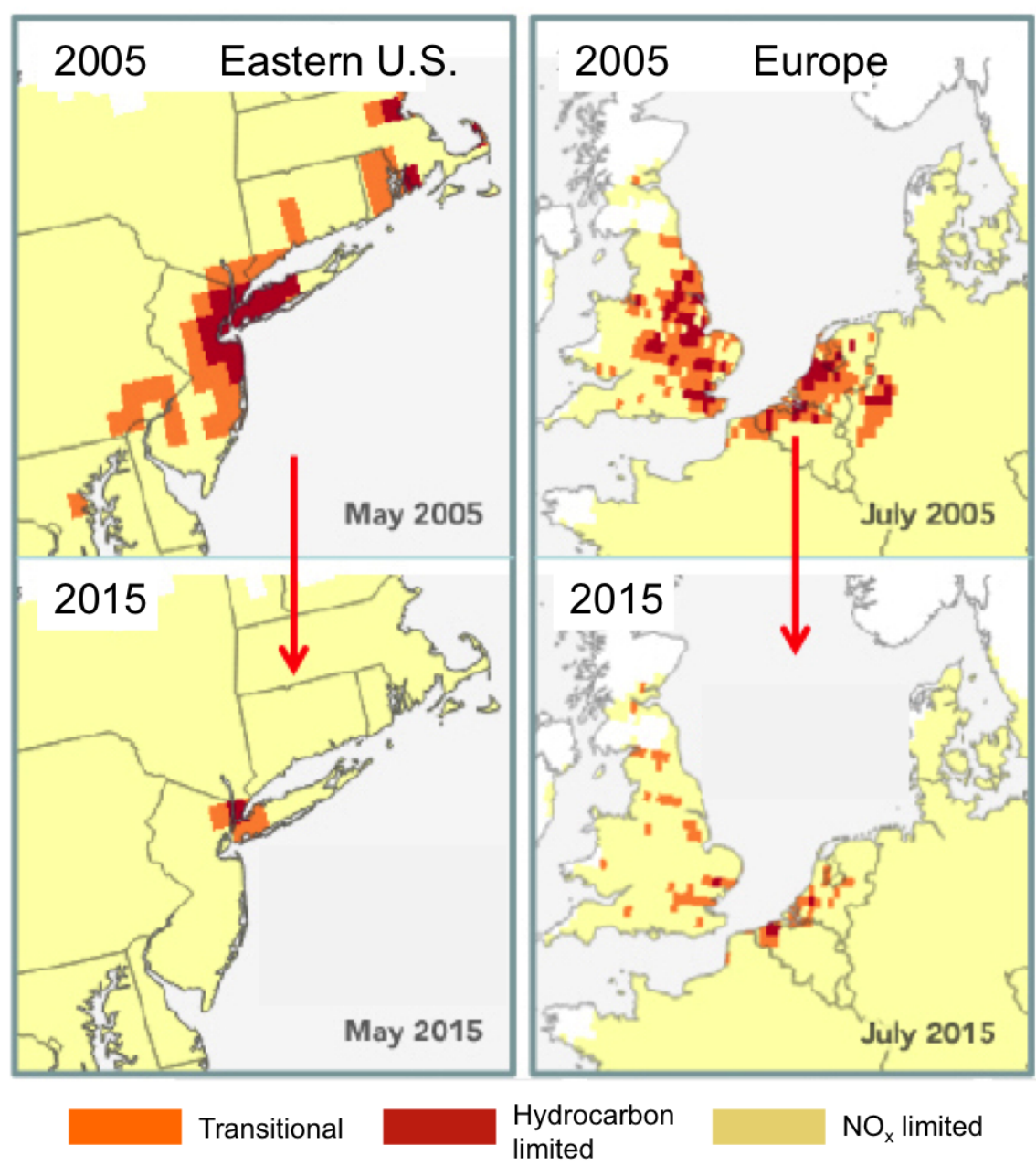

Figure 1.5: Regions of Europe and Eastern U.S classified as $\mathrm{NO}_{x}$ limited, hydrocarbon limited and transitional based on the $\mathrm{NO}_{2}$ and $\mathrm{HCHO}$ ratio measured from the OMI satellite for 2005 (upper row) and 2015 (lower row) (modified from AURA Science webpage https://aura.gsfc.nasa.gov/science/feature-20171228b.html).

monitored from space (e.g. Lamsal et al. (2011)).

Satellite measurements have been used to link changes in emissions to environmental regulations and economic activities. Castellanos and Boersma (2012) analysed daily tropospheric $\mathrm{NO}_{2}$ columns over Europe measured by the OMI satellite and linked the decrease in $\mathrm{NO}_{2}$ levels between 2004 and 2010 to environmental regulations, with several regions showing an increase due to economic development. They also showed that in 2009, the global recession accelerated the decrease in $\mathrm{NO}_{2}$ compared to other years. Duncan et al. (2016) used OMI measurements to analyse urban concentrations of $\mathrm{NO}_{2}$, and linked the decrease in $\mathrm{NO}_{2}$ levels to reasons varying from environmental regulations (e.g. Europe) to civil unrest (e.g. Syria). In contrast, they associated higher $\mathrm{NO}_{2}$ levels to locations of intensive energy activity and to the economic growth of several Asian 
and tropical cities. Jiang et al. (2018) used satellite measurements together with ground based observations to asses the changes in pollutant emissions. They found that in the 2011-2015 period the reduction in $\mathrm{NO}_{x}$ emissions was negligible compared to 2005-2009, completely opposite to the emission reduction reported by the EPA's emission inventory. This discrepancy could be attributed to an increase in the contribution of industrial and area sources, off-road mobile sources and a slower decrease in $\mathrm{NO}_{x}$ emissions from diesel vehicles. This shows the relevance of using satellite measurements to estimate emissions (i.e. a top-down approach) and to validate the emissions reported in the inventories (the bottom-up approach).

The use of data assimilation techniques together with satellite measurements allows to have a global estimate of the magnitude and distribution of emissions from daily to decadal time scales. Miyazaki et al. (2017) obtained top-down $\mathrm{NO}_{x}$ global emission estimates from 2005 to 2014 by assimilating satellite measurements of several species in a global chemistry transport model. The study showed a strong increase in $\mathrm{NO}_{x}$ emissions over India and Middle East, and a decrease over the U.S., southern Africa and western Europe. In China, a positive trend between 2005-2011 was followed by a decrease in $\mathrm{NO}_{x}$ emissions after 2011 . In this study, they assimilated not only satellite measurements of $\mathrm{NO}_{2}$ but also $\mathrm{O}_{3}, \mathrm{CO}$ and $\mathrm{HNO}_{3}$ from different satellites to better constraint tropospheric chemistry. This multiconstituent data assimilation approach shows a better agreement of simulated species with independent observations, as well as a reduction in simulation errors (Miyazaki et al., 2018).

Air pollution is not just a problem at local or regional scales. Global transport of pollution can impact air quality at local scales far away from the source. An example of this trans-boundary transport of air pollution problem is ozone. Verstraeten et al. (2015) showed that even though precursor emissions decreased over western U.S., free tropospheric ozone did not show the same tendency due to long-range transport of ozone and its precursors from China and the increase in the stratosphere-troposphere ozone transport. The use of satellite measurements of $\mathrm{NO}_{2}$ by OMI and $\mathrm{O}_{3}$ by TES (Tropospheric Emission Spectrometer) were fundamental in this study to understand a global scale and long term problem. OMI $\mathrm{NO}_{2}$ columns indicated an increase in $\mathrm{NO}_{x}$ emissions between 2005 and 2010 of about $20 \%$ over China and a similar decrease over western U.S. TES measurements revealed a significant $\mathrm{O}_{3}$ increase over China and a modest increase in western U.S., which pointed to long range $\mathrm{O}_{3}$ transport as an extra source that could explain this increase. The assimilation of satellite measurements of $\mathrm{OMI} \mathrm{NO}_{2}$ and TES $\mathrm{O}_{3}$ in models has also contributed to a better attribution of the sources that affect western U.S. due to transport from the Eastern Pacific (e.g. Huang et al. (2015)).

The paragraphs above give an overview of various successful applications of $\mathrm{NO}_{2}$ satellite measurements, mostly related to air quality and emission quantification. Many of 
these applications have had a great impact as they show how satellite measurements can serve society in many ways (e.g. as a basis for policymakers to make the right decisions for emission controls to be successful). Still, $\mathrm{NO}_{2}$ measured from space is an indirect measurement, with multiple assumptions and error sources, as the reader will learn throughout this thesis. So even though trace gas satellite retrievals have improved over the last decades, there is a constant need to improve the retrieval algorithms for a better characterization of the measurements and to reduce and better characterize the uncertainties associated with it. Satellite data users need to have confidence that the quality of the measurements is such that fits the fitness of purpose of their applications.

\subsection{Satelite remote sensing of atmospheric composi- tion in the UV-Vis}

The monitoring of atmospheric composition from space has developed vastly in the last two decades, together with the rapid increase of the need of having accurate information to understand and mitigate air pollution and climate change effects. Since satellite measurements have become a very useful tool for atmospheric composition monitoring, it has also become a priority for data producers to provide quality assured satellite data records, with a more complete and understandable information on the processing chain, uncertainties and the fitness-for-purpose of the satellite data products. Ultimately, satellite measurements have to be reliable and robust enough so they can be used by agencies, stakeholders and policymakers.

From the mid 60's to the 90's, the National Oceanic and Atmospheric Administration (NOAA) and the National Aeronautics and Space Administration (NASA) from the United States were leading the programs to monitor atmospheric composition with the Nimbus and NOAA 4th generation satellite programs. The Total Ozone Mapping Spectrometer (TOMS) sensor onboard Nimbus-7 satellite was the first to monitor stratospheric $\mathrm{O}_{3}$ in the early 80's. In the 90's, the first European program for monitoring Earth's atmospheric chemical composition started with the European Remote Sensing (ERS) missions. Among the sensors in the ERS-2 satellite launched in 1995 was the Global Ozone Monitoring Experiment (GOME), aimed at monitoring ozone, trace gases and aerosols. The Environmental Satellite (ENVISAT) was launched in 2002 with the Scanning Imaging Absorption Spectrometer for Atmospheric Cartography (SCIAMACHY) satellite on-board, a mission also lead by the European Space Agency (ESA). On board the European Organisation for the Exploitation of Meteorological Satellites (EUMETSAT) and ESA's Meteorological Operational Satellite (Metop) - A (2006), B (2012) and C (2018), the GOME-2 instrument is included as a follow-up of GOME. The Dutch-Finish Ozone Mon- 
itoring Instrument (OMI) on board NASA's Aura satellite was a major improvement in spatial resolution compared to its predecessors. The high spatial resolution $\left(13 \times 24 \mathrm{~km}^{2}\right.$ at nadir) and the wide swath $(2600 \mathrm{~km})$ allow daily full global coverage. Since its launch in 2004, it has provided high quality data of atmospheric trace gases (Levelt et al., 2018). Figure 1.6 gives an overview of the past, current and future satellite sensors that measure $\mathrm{NO}_{2}$ columns in the atmosphere since GOME was launched in 1995.

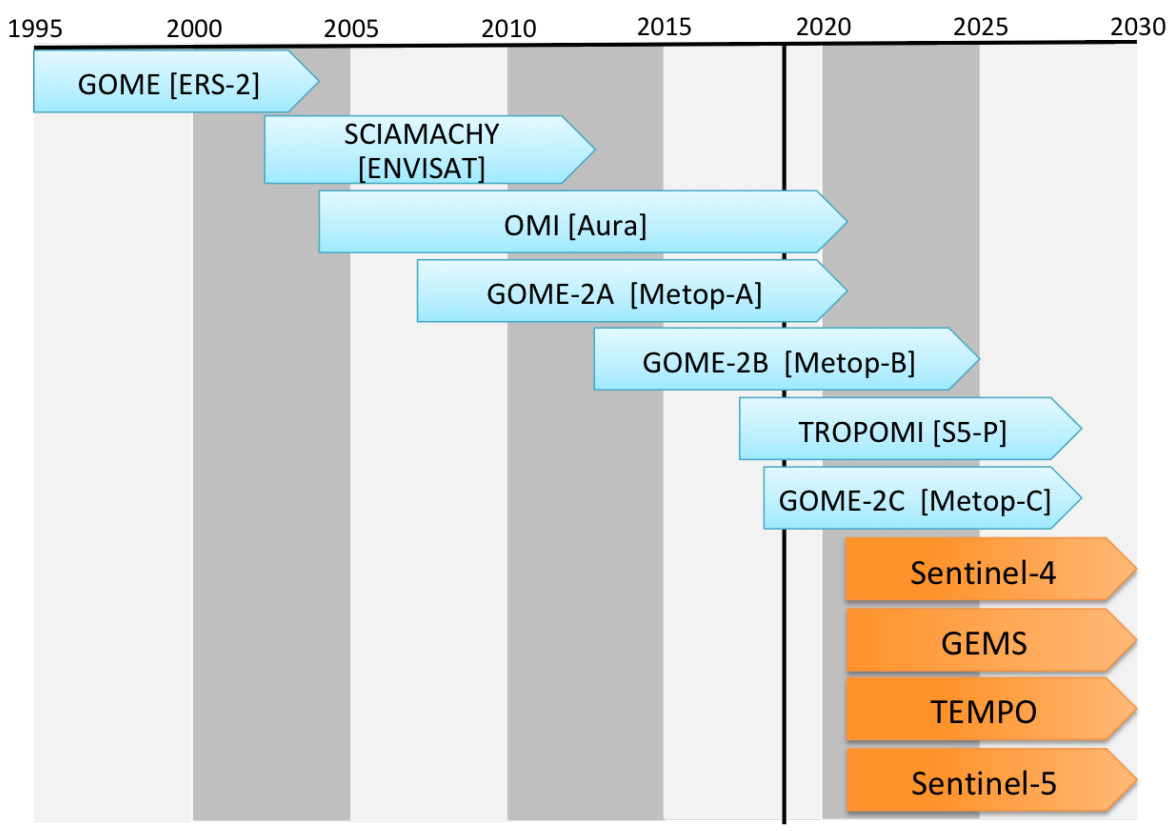

Figure 1.6: Timeline of UV/Vis polar orbiting and geostationary satellite instruments that measure $\mathrm{NO}_{2}$ columns since 1995. Launch date for future sensors (in orange) still needs to be determined.

A joint initiative between the European Commission and the ESA are the Sentinel missions as part of the Copernicus Earth Observation programme. Land, ocean and atmosphere will be monitored by this constellation of satellites. The Tropospheric Monitoring Instrument (TROPOMI) is the instrument on the payload of the Sentinel-5 Precursor (S5-P) satellite, aimed at monitoring atmospheric composition and chemistry. Since its launch in October 2017, it is providing unprecedented high-resolution (up to $7 \times 3.5 \mathrm{~km}^{2}$ ) measurements of $\mathrm{NO}_{2}, \mathrm{CO}, \mathrm{CH}_{4}, \mathrm{O}_{3}, \mathrm{SO}_{2}$ and $\mathrm{HCHO}$ among others. All the satellites mentioned above are "low Earth orbit" (LEO) satellites in Sun-synchronous orbits. These satellites make global measurements, and the overpass at a given latitude is always at the same local time. For example, OMI and TROPOMI cross the Equator around 13:30 LT, while GOME, SCIAMACHY and GOME-2 cross(-ed) it around 10:30 LT. Geostationary satellites have a fixed position relative to the Earth, and their field-of-view covers a limited area. The main advantage of geostationary satellite instruments is the high temporal resolution that allows the monitoring of diurnal pattern of pollutants, which is particularly interesting for short-lived species like $\mathrm{NO}_{2}$. There are three geostationary 
satellites that will be launched in the coming years that will host different sensors to measure atmospheric composition over Europe (Sentinel - 4), over North America (Tropospheric Emissions: Monitoring of Pollution TEMPO) and over Asia (Geostationary Environmental Monitoring Spectrometer GEMS). This constellation of satellites brings new challenges for improving satellite measurements, as important aspects like viewing geometry and overpass times will change and these need to properly be accounted for. These satellites will provide a new perspective in the understanding of atmospheric composition from remote sensing observations.

\section{Measurement principles}

Remote sensing instruments discussed above measure backscatter radiation in different spectral ranges, so they do not directly measure atmospheric composition. Incoming radiation from the Sun, when travelling through the atmosphere and back to the satellite, interacts with the different elements of the atmosphere (e.g. clouds, aerosols, trace gases) and the surface. The radiance spectra measured by the satellite contain information on the components that absorb and scatter light, and when we apply a retrieval algorithm to the measured reflectance spectrum we can obtain information (e.g. characteristics and abundance) about these different components. The measured reflectance spectrum is defined as the ratio of the Earth's backscatter radiance spectrum $I(\lambda)$ (times $\pi$ ) and a reference spectrum, typically the direct solar irradiance spectrum at the top of the atmosphere $E_{0}(\lambda)$ multiplied by the cosine of the solar zenith angle $\mu_{0}$ :

$$
R(\lambda)=\frac{\pi I(\lambda)}{\mu_{0} E_{0}(\lambda)}
$$

Figure 1.7 shows a solar irradiance and Earth radiance spectrum measured by the OMI instrument in the UV/Vis (280-500 nm), and the ratio of both spectra. The differences between solar irradiance and Earth radiance spectrum are due to absorption and scattering by the different atmospheric constituents. Strong spectral signatures due to ozone absorption for wavelengths below $340 \mathrm{~nm}$ are visible in Fig. 1.7a,b and at larger wavelengths Rayleigh scattering by molecules is the dominant extinction process. The inset in Fig. 1.7b shows the spectral absorption structures of $\mathrm{NO}_{2}$ enlarged around 440 $\mathrm{nm}$. These $\mathrm{NO}_{2}$ absorption features are much weaker than the ozone absorption features around $300 \mathrm{~nm}$. This is because the optical thickness of $\mathrm{NO}_{2}$ around $440 \mathrm{~nm}$ is on the order of 0.005 , more than 1000 times smaller than the ozone optical thickness around 300 $\mathrm{nm}$.

Atmospheric retrievals make use of spectral fitting techniques to derive the concentration of a specific trace gas integrated along the average atmospheric light path of the 

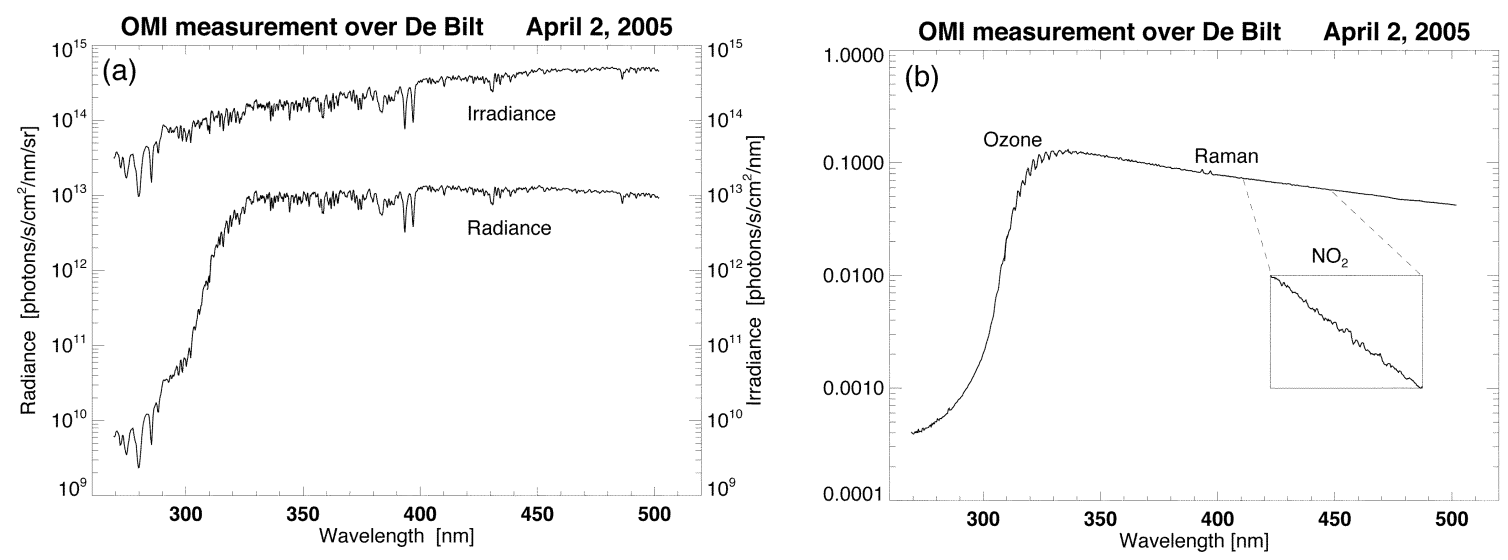

Figure 1.7: (a) Solar irradiance and Earth radiance spectrum measured by OMI on a clear sky day over The Netherlands and (b) the ratio of both spectra in (a) (from Levelt et al. (2006)).

backscatter radiation (the so-called slant column density (SCD)). Spectral absorption structures like the ones of $\mathrm{NO}_{2}$ in the UV/Vis makes it possible to apply Differential Optical Absorption Spectroscopy (DOAS) technique (Platt, 2017). The DOAS technique is based on the Beer-Lambert law that describes the attenuation of light in the atmosphere due to scattering and absorption:

$$
I(\lambda)=I_{0}(\lambda) \exp \left[\left(-\sum_{\mathrm{i}} \sigma_{\mathrm{i}}(\lambda) \cdot \mathrm{L} \cdot \mathrm{c}_{\mathrm{i}}\right)\right]
$$

Equation 1.2 states that the attenuation of the (initial) intensity $I_{0}$ at the top of the atmosphere is proportional to the absorption cross-section $\sigma_{i}$, the light path length $L$ and the concentration of the absorber $i$. In DOAS, least squares methods are used to match the observed spectral signatures to a modelled reflectance spectrum that takes into account molecular absorption spectral features and other extinction processes in a specific fitting window. The modelled reflectance separates spectral absorption features into low and high frequency contributions. Low frequency (i.e. spectrally smooth) features are included in a low order polynomial $P(\lambda)$, that accounts for the smooth absorption and scattering features such as Rayleigh and Mie scattering and surface reflection. The high frequency contribution is caused by absorption by atmospheric trace gases like $\mathrm{NO}_{2}$, and is used for the retrieval of the slant column density $N_{s}$. Equation 1.2 is then expressed as:

$$
I(\lambda)=I_{0}(\lambda) \exp \left[-\sum_{\mathrm{i}=1} \sigma_{\mathrm{i}}(\lambda) N_{\mathrm{s}, \mathrm{i}}-\sum_{\mathrm{P}} \mathrm{a}_{\mathrm{P}} \lambda^{\mathrm{P}}\right]
$$


Other effects (e.g. instrumental features) might be also accounted for in the modelled reflectance. The Ring effect, which is due to inelastic rotational Raman scattering by $\mathrm{N}_{2}$ and $\mathrm{O}_{2}$ molecules in the atmosphere, is taken into account by including a pseudo absorber with a synthetic Ring spectrum. Figure 1.8 shows an example of TROPOMI's measured reflectance for the retrieval of $\mathrm{NO}_{2}$ and the modelled reflectance obtained with the DOAS fit using the following expression:

$$
R_{\text {mod }}=P(\lambda) \cdot \exp \left[\sum_{\mathrm{i}=1} \sigma_{\mathrm{i}}(\lambda) N_{\mathrm{s}, \mathrm{i}}\right] \cdot\left(1+C_{\text {Ring }} \frac{I_{\text {ring }}(\lambda)}{\mathrm{I}_{0}(\lambda)}\right)
$$

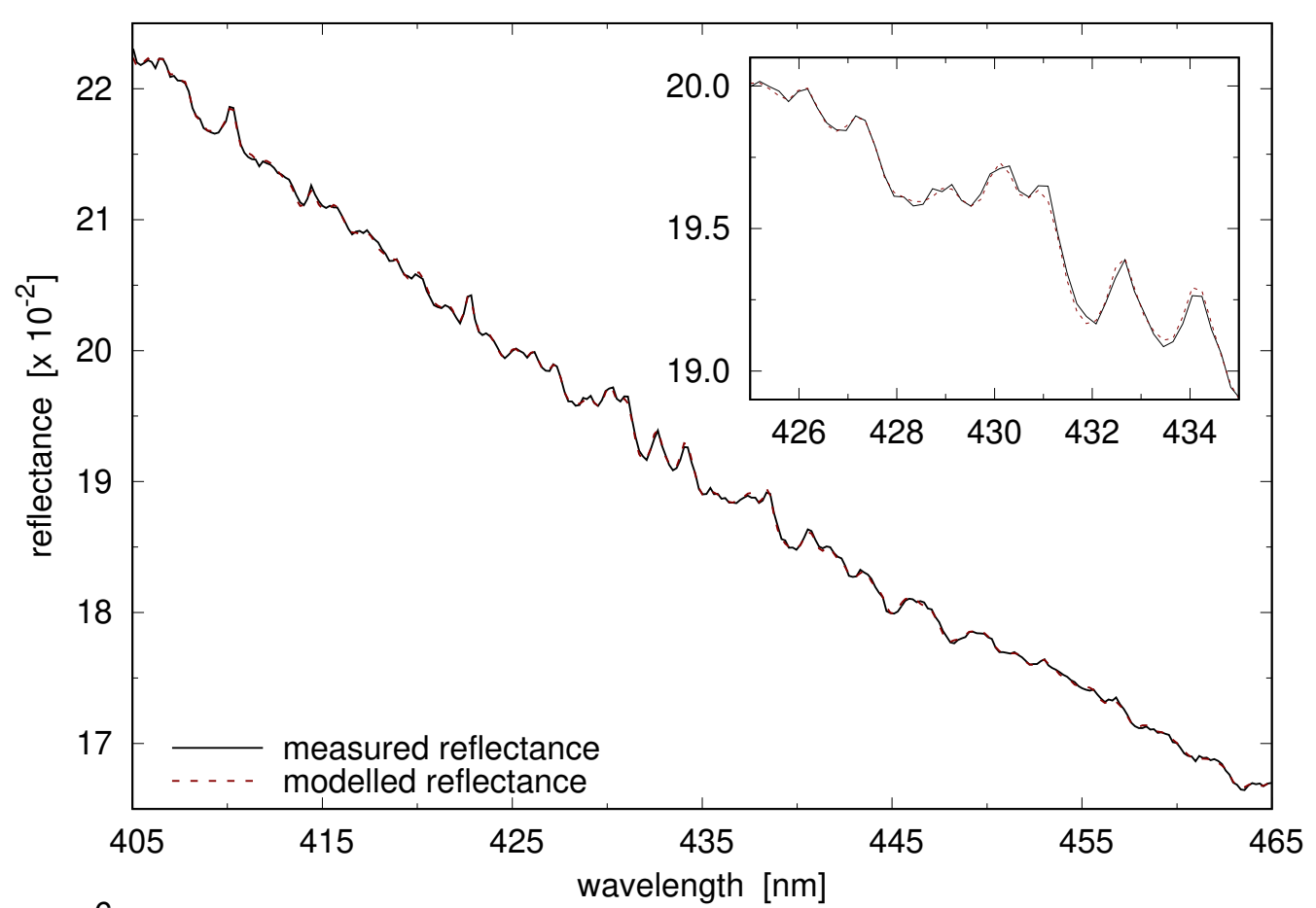

Figure 1.8: Reflectance spectrum measured by TROPOMI on 4 July 2018 and the modelled reflectance spectrum obtained with the DOAS fit (adapted from van Geffen et al. (2018)).

After the spectral fitting has been applied to obtain the slant column density, it is converted to a vertical column density (VCD) by taking into account the radiative transfer properties of the atmosphere. For this conversion, we use a radiative transfer model to calculate the air mass factor (AMF), which represents the length of the average light path through the atmosphere. The AMF calculation assumes knowledge on the measurement geometry, and a priori knowledge of the state of the atmosphere, so it requires external information on geophysical variables such as surface albedo, surface height, and vertical profile of the trace gas. As the interaction of radiation with the atmosphere is sensitive to the vertical distribution of the absorber in the atmosphere, the AMF depends on the vertical distribution of the trace gas. Palmer et al. (2001) proposed a formulation 
to decouple the dependency of the AMF on the vertical distribution of $\mathrm{NO}_{2}$ by using altitude-dependent (box-) air mass factors that account for the vertical sensitivity of the measurement. Then the total AMF can be expressed as sum of the box-AMF of each layer multiplied by the trace gas vertical profile (e.g. calculated with a chemistry transport model or from aircraft measurements) weighted by the vertical column. This formulation requires that the absorber is optically thin (optical thickness $\tau_{\text {gas }} \ll 1$ ) so the light path does not depend on the absorber concentration. For high pollution events, and when using a wider and red-shifted fitting window, the $\mathrm{NO}_{2}$ atmospheric column is no longer optically thin and AMFs (i.e. measurement sensitivity) decrease. In this case of optically thick absorber, the AMF depends on the amount of $\mathrm{NO}_{2}$ assumed in the radiative transfer modelling and a correction factor is applied based on the relation of the slant column and the AMF for different assumed $\mathrm{NO}_{2}$ columns (S5P Verification Team, 2015).

The calculation of the AMF, and thus the vertical column density, implicitly depends on the a priori trace gas profile shape and this dependency is a source of systematic error in the retrieval. The averaging kernels (AKs) represent how the retrieved VCD changes when the absorber concentration changes at a certain level. AKs are calculated as the ratio of the box-AMF of layer $l$ and the total AMF, and they provide the relation between the vertical column and the slant column density without the influence of the a priori profile (Eskes and Boersma, 2003). The application of the $\mathrm{AKs}$ to $\mathrm{NO}_{2}$ vertical profiles removes the AMF dependency on the a priori trace gas profile used in the retrieval, which is essential to properly interpret satellite retrievals and to compare satellite measurements to chemistry transport models and measured profiles in an independent way. Boersma et al. (2016b) showed that larger errors when doing model to satellite comparisons may occur when the comparison is done without using averaging kernels. Averaging kernels can be used to recalculate the air mass factor to use an a priori profile that is more suitable for a particular model to satellite comparison, e.g. from aircraft measurements (Bucsela et al. (2008), Boersma et al. (2008)). Averaging kernels are also used in data assimilation of satellite retrievals to remove the retrieval dependency on the a priori profile (e.g. Miyazaki et al. (2017)), and in this way the retrieval uncertainty due to the a priori profile can be neglected. Errors related to the use of other ancillary data (i.e. clouds, albedo, aerosols and surface reflectivity) remain even after using the AKs.

The presence of residual clouds and aerosols is a big challenge for retrievals in the $\mathrm{UV} / \mathrm{Vis}$, as they modify the photon path length. The net effect in the $\mathrm{NO}_{2}$ retrieval depends on the location of the $\mathrm{NO}_{2}$ relative to the cloud or aerosol layer (e.g. Leitao et al. (2010)). If the $\mathrm{NO}_{2}$ is located below clouds, there is a reduced sensitivity as less photons will travel through the air mass below the cloud. In contrast, the sensitivity to $\mathrm{NO}_{2}$ will be enhanced if the trace gas is located within or above the cloud or aerosol layer. 
The independent pixel approximation (IPA) implicitly accounts for these physical effects, and calculates the AMF as a linear combination of cloudy and clear components of the AMF, weighted by the fraction of satellite-measured radiance that originates from the cloudy part of the pixel (Martin et al., 2002). The cloud information (cloud pressure and cloud fraction) to calculate the cloudy AMF is taken from a cloud retrieval applied (if possible) to measurements from the same satellite instrument, using as much as possible the same assumptions on surface reflectance. Another approach is to consider clear-sky AMFs for scenes with a sufficiently small cloud fraction, without applying any correction. The differences between the IPA AMF and clear-sky AMF for scenes with small cloud fraction will be discussed in detail in Chapter 3.

For aerosols, typically an implicit or explicit aerosol correction is applied in the UV/Vis trace gas retrievals. The implicit correction is based on the fact that the effective cloud parameters used in the calculation of the AMF are sensitive to the presence of aerosols (e.g. Boersma et al. (2011)), and therefore the AMF implicitly contains the effects of aerosols. There is also the possibility to explicitly correct for aerosols by introducing aerosol optical properties in the radiative transfer simulations (Lin et al., 2014), but for this accurate information of the type, vertical distribution and amount of aerosols is required. The implicit aerosol correction via the effective cloud parameters works well for relatively small scattering aerosols, and for scenes with moderate aerosol optical thickness (AOT below 0.6) (e.g. Boersma et al. (2011), Castellanos et al. (2015)). These are the cases when the cloud algorithm is most sensitive to aerosols, as the increased TOA reflectance due to aerosol scattering is interpreted as a cloud and accounted for in the retrieved effective cloud parameters. For high optical thickness and absorbing aerosols, there are compensating mechanisms between the effect of smaller effective cloud pressures and smaller effective cloud fractions occurring in the implicit aerosol correction (e.g. Castellanos et al. (2015)). In those cases, the explicit aerosol correction might be the most suitable treatment of aerosols in the $\mathrm{NO}_{2}$ retrieval, as is the case for high pollution events in China (e.g. Lin et al. (2015), Liu et al. (2019)). The errors due to the treatment of aerosols are rather complex and very dependent on the optical and physical properties of the aerosols and the reflecting surface (e.g. Chimot et al. (2018)). Actually, the choice of the aerosol correction is one of the biggest source of uncertainty in the AMF calculation, as shown in Chapter 3 .

\subsection{Radiative transfer in the atmosphere}

Radiative transfer modelling in the atmosphere is key in satellite trace gas measurements. Because radiation is modified by the different extinction processes when passing through the atmosphere and by reflection at the surface, it is essential to model these processes 
properly to have accurate satellite measurements. In the UV/Vis spectral range, extinction processes are of central importance. Beer-Lambert's law describes extinction due to scattering and absorption. In the Beer-Lambert law, the extinction rate is determined by the cross sections of each of the absorption and scattering processes and the viewing geometry. The absorption cross sections of the different molecules strongly depend on the wavelength and they are normally obtained from laboratory measurements. The scattering phase function describes the scattering processes, and it represents the angular distribution of the scattered radiation intensity, which depends on the direction of the incident light and the scattering angle. The scattering processes depend on the wavelength and on the size of the particles that interact with the radiation. In Fig. 1.9 the blue sky is an example of scattering by air molecules (i.e. by molecules that have a size in the order of a nm like nitrogen and oxygen) which is described by Rayleigh scattering. The Rayleigh scattering cross section is proportional to $\lambda^{-4}$, which means that shorter wavelengths (i.e. blue light) are scattered more efficiently, making the sky appear blue. Rayleigh scattering is also stronger in the lower part of the troposphere where the density of air molecules is higher, and that is why satellite measurements are less sensitive to trace gases close to the surface. Clouds in Fig. 1.9 are an example of scattering by larger particles like cloud droplets (around $10 \mu \mathrm{m}$ radius), described by Mie scattering. Mie scattering has a slight dependency on wavelength (as relative to Rayleigh scattering), and that is why we see clouds in white color. In atmospheric applications typically a size distribution is needed to describe the phase function for Mie scattering.

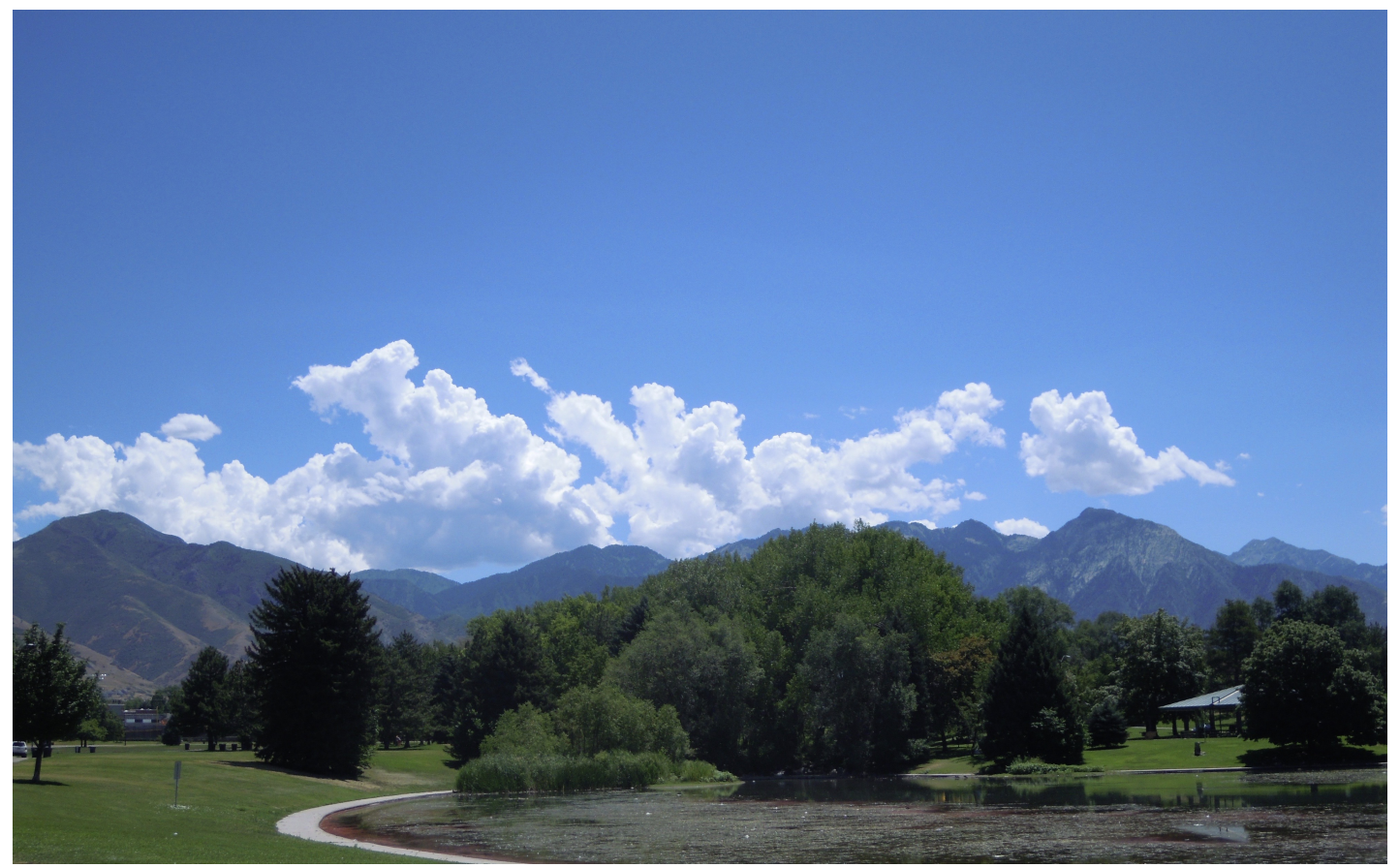

Figure 1.9: Example of Rayleigh scattering (blue sky) and Mie scattering (white clouds). Photo taken by Alba Lorente in Salt Lake City (Utah), in August 2017. 
The radiative transfer equation (RTE) is the mathematical formulation that describes the loss and gain of radiation due to all the processes described above. The RTE is usually discretized assuming the atmosphere as a multi-layer stratified medium, where each layer is optically uniform. The RTE results in a complex ${ }^{2}$ equation, and to solve it complex mathematical methods are needed. A radiative transfer model (also known as forward model) calculates the radiation field for a given atmospheric state. This is what is typically done in $\mathrm{UV} / \mathrm{V}$ is retrievals to compute the air mass factor given the atmospheric state and boundary conditions. In Chapter 3, we compare four radiative transfer models to establish the error of forward models for UV/Vis retrievals, as each of these RTMs use different methods to solve the radiative transfer equation that could lead to different estimation of atmospheric radiation fields. SCIATRAN and VLIDORT (Vector-LInearized Discrete Ordinate Radiative Transfer) use mathematical methods to solve the system of differential equations to which the RTE is reduced after applying a quadrature method to solve the integrals. DAK (Doubling-Adding KNMI) uses the doubling-adding method, which calculates the transmission and reflection of individual homogeneous atmospheric layers and by adding these layers on top of each other, it yields to the reflection and transmission of the combined layers. McArtim (Monte Carlo Atmospheric Radiative Transfer Inversion Model) uses statistical approaches to calculate probability distributions for the different radiative transfer processes. These four models also describe differently the sphericity of Earth's atmosphere, a characteristic that is relevant in the treatment of the scattering processes in the atmosphere.

One of the boundary values necessary to solve the radiative transfer equation is the upward radiation field at the surface, i.e. the surface reflection. The reflecting properties of the surface can be considered isotropic (i.e. equal in all directions) or anisotropic (i.e. angular dependent). In the isotropic case the surface is considered to be a Lambertian reflector, so the intensity of the reflected light does not depend on the incident nor the reflected direction. However, surfaces reflect light differently in each direction, so anisotropy is a fundamental physical property of the surface reflectance. The angular distribution of the reflected light by a surface is represented mathematically by the bidirectional reflectance distribution function (BRDF) (Nicodemus et al., 1992). The assumption of Lambertian reflector is widely used in the UV/Vis satellite retrieval community, justified by the fact that surface BRDF effects are likely to smooth out over the large pixels. However, for smaller pixel size (like in TROPOMI) this assumption is no longer valid. In order to fully represent the geometry-dependent surface scattering properties in cloud and trace gas retrievals, the surface BRDF has to replace the isotropic Lambertian albedo. We show in Chapter 4 that surface BRDF effects are stronger for retrievals in the near-infrared, as Rayleigh scattering in the visible partly smooths out these effects.

\footnotetext{
${ }^{2}$ Integro-differential equation, i.e. equation that contains integrals and derivatives.
} 


\subsection{Thesis outline}

This thesis builds up from the motivation to improve $\mathrm{NO}_{2}$ retrievals and to quantify the uncertainties associated with it. We focus here on improving the air mass factor calculation and establishing the error of the radiative transfer in the retrieval, and we properly characterize the surface reflectance for cloud and $\mathrm{NO}_{2}$ retrievals. The thesis culminates with the application of the improved TROPOMI $\mathrm{NO}_{2}$ retrievals to infer daily $\mathrm{NO}_{x}$ emissions at urban scales.

In the Quality Assurance for Essential Variables (QA4ECV) project, we developed a community $\mathrm{NO}_{2}$ and $\mathrm{HCHO}$ retrieval algorithm based on best practices of different state of the art retrieval algorithms, applicable to four different satellite sensors (GOME, SCIAMACHY, GOME-2 and OMI, see Fig. 1.6) to obtain more than 20 years of harmonized $\mathrm{NO}_{2}$ measurements. In Chapter 2 we explain the build up of each step of the QA4ECV $\mathrm{NO}_{2}$ retrieval algorithm. In order to select the best approach for the AMF calculation step, we will identify in Chapter 3 characteristics and assumptions of the radiative transfer modelling part that should be improved. We will establish the error of forward models for UV/Vis retrievals and improve the calculation of the altitude-dependent AMF for $\mathrm{HCHO}$ and $\mathrm{NO}_{2}$ retrievals, and implement those in the QA4ECV algorithm. The research questions answered in Chapter 3 can be summarized as follows:

- How can we improve the air mass factor calculation process?

- What are the main sources of the AMF structural uncertainty, and can we quantify these uncertainties for every step of the calculation?

The representation of the surface as a Lambertian reflector is still an issue in $\mathrm{NO}_{2}$ and cloud retrievals. In Chapter 4 we will asses the surface reflectance anisotropy effects on surface Lambertian Equivalent Reflector (LER) climatologies, on cloud and trace gas retrievals, covering all the steps from TOA reflectance simulation to the calculation of tropospheric AMFs. We demonstrate that surface BRDF effects need to be coherently considered to accurately retrieve clouds and $\mathrm{NO}_{2}$ from satellites. The research questions that will be addressed in Chapter 4 can be summarized as follows:

- How accurate is the use of Lambertian surface albedo for backward and forward scatter viewing geometries from different satellite instruments?

- How can we account for surface reflectance anisotropy in the radiative transfer model DAK?

- How does surface reflectance anisotropy affect cloud retrievals and $\mathrm{NO}_{2}$ retrievals via clear sky and cloudy sky contributions to the tropospheric AMFs? 
The recently launched TROPOMI satellite instrument inherited most of the improvements of the QA4ECV retrieval algorithm. In Chapter 5 we explore the capabilities of TROPOMI's unprecedented high resolution $\mathrm{NO}_{2}$ measurements to infer the magnitude and spatial distribution of daily $\mathrm{NO}_{x}$ emissions from megacities. The research questions addressed in Chapter 5 can be summarized as follows:

- How is the quality of the TROPOMI $\mathrm{NO}_{2}$ retrieval algorithm and of the high resolution $\mathrm{NO}_{2}$ measurements?

- Can we estimate daily $\mathrm{NO}_{x}$ emissions from TROPOMI $\mathrm{NO}_{2}$ measurements focusing on the build up of pollution within the city?

- How robust is the method applied to a megacity like Paris? 



\section{QA4ECV $\mathrm{NO}_{2}$ retrieval algorithm: on the way to TROPOMI}

This chapter includes material from:

Boersma et al. (2018): Improving algorithms and uncertainty estimates for satellite $\mathrm{NO}_{2}$ retrievals: Results from the Quality Assurance for Essential Climate Variables (QA4ECV) project.

Lorente et al. (2016): Air Mass Factor Calculation Best Practices and Recommendations, Sect. 2.5 of QA4ECV Deliverable 4.2 version 1.0.

Müller et al. (2016): Recommendations (scientific) on best practices for retrievals for Land and Atmosphere ECVs. Deliverable 4.2 version 1.0. 


\subsection{QA4ECV for $\mathrm{NO}_{2}$}

Nitrogen dioxide $\left(\mathrm{NO}_{2}\right)$, together with formaldehyde (HCHO), sulfur dioxide $\left(\mathrm{SO}_{2}\right)$, and carbon monoxide (CO), was included by the Global Climate Observing System (GCOS) in the Essential Climate Variables (ECV) framework as a precursor of ozone and aerosols. ECVs are variables that contribute to the characterization of Earth's climate system and its changes; ozone and aerosol precursors were considered due to their importance to understand past, present and future climate, and because long term record and global monitoring of these precursors (i.e. $\mathrm{NO}_{2}$ ) is achievable with existing and future observing systems (Bojinski et al., 2014). In order to serve this purpose, a consistent and reliable multi-sensor data record of $\mathrm{NO}_{2}$ is needed. Furthermore, long, consistent time series of $\mathrm{NO}_{2}$ are necessary for trend analysis of the $\mathrm{NO}_{2}$ tropospheric columns (e.g. van der A et al. (2008)) and also of the $\mathrm{NO}_{x}$ emissions that can be inferred from inverse modelling analysis and data assimilation systems that use $\mathrm{NO}_{2}$ from multiple satellites (e.g. Miyazaki et al. (2017)). These studies, besides the consistent $\mathrm{NO}_{2}$ data record, also require a complete understanding and characterization of the uncertainties to apply a more realistic constraint to the modelled fields.

The aim of the 2014-2018 European Union (EU) Seventh Framework (FP7) project Quality Assurance for Essential Climate Variables (QA4ECV, www.qa4ecv.eu) was to create a harmonized multi decadal record of $\mathrm{NO}_{2}$ (and also $\mathrm{HCHO}$ and $\mathrm{CO}$ ) satellite measurements. In QA4ECV we have developed a community best practice retrieval algorithm, and by applying it to the GOME (1995-2003), SCIAMACHY (2002-2012), OMI (2004-2017) and GOME-2A (2007-2016) sensors, we have retrieved more than 20 years of quality-assured data in a consistent way, both at algorithm level and across timescales. Apart from developing an improved retrieval algorithm, delivering a detailed uncertainty evaluation and assessing the quality of the data product were also central objectives of QA4ECV. Some of the developments made to create the $\mathrm{NO}_{2}$ QA4ECV retrieval algorithm have been adopted in the $\mathrm{NO}_{2}$ retrieval of the Sentinel 5 Precursor (S5P) TROPOMI instrument (van Geffen et al., 2018) and have served as a benchmark for the preparation of its successors Sentinel 4 and Sentinel 5.

Global $\mathrm{NO}_{2}$ measurements started with the Global Ozone Monitoring Experiment (GOME) instrument (Burrows et al., 1999), launched in 1995. SCIAMACHY (Scanning Imaging Absorption spectroMeter for Atmospheric CHartographY) instrument (Bovensmann et al., 1999) was launched in 2002. GOME-2 on board Metop-A was launched in 2006 as a continuation of the long-term monitoring started by GOME and SCIAMACHY and a second identical instrument was launched in 2012 on board Metop-B (GOME-2B) (Munro et al., 2016). Metop-C is the last of the Metop satellites, and it has joined the tandem in November 2018, also with a GOME-2 instrument on its payload. OMI (Ozone 
Monitoring Instrument) was launched in 2004 and it is still operational (Levelt et al., 2018). TROPOMI on board Sentinel-5P was launched in October 2017 (Veefkind et al., 2012). Table 2.1 provides an overview of the characteristics from TROPOMI and the sensors on which QA4ECV retrieval algorithm has been applied to produce the $\mathrm{NO}_{2}$ data record.

Table 2.1: Overview of the main characteristics relevant for $\mathrm{NO}_{2}$ retrieval of the four sensors used in QA4ECV and TROPOMI.

\begin{tabular}{|c|c|c|c|c|}
\hline & $\begin{array}{l}\text { Local Equator } \\
\text { crossing time }\end{array}$ & $\begin{array}{l}\left.\text { Resolution* }{ }^{*} \mathrm{~km}^{2}\right] \\
\text { Swath }[\mathrm{km}]\end{array}$ & $\begin{array}{l}\text { Global } \\
\text { coverage }\end{array}$ & $\begin{array}{l}\text { Spectral } \\
\text { range }[\mathrm{nm}]\end{array}$ \\
\hline $\begin{array}{l}\text { GOME } \\
(1995-2003)\end{array}$ & 10:30 hrs & $\begin{array}{l}320 \times 40 \\
960\end{array}$ & 1-3 days & $240-790$ \\
\hline $\begin{array}{l}\text { SCIAMACHY } \\
(2002-2012)\end{array}$ & 10:30 hrs & $\begin{array}{l}30 \times 60 \\
960\end{array}$ & 3-6 days & $220-2380$ \\
\hline $\begin{array}{l}\text { GOME-2A } \\
(2006-2016)\end{array}$ & 10:30 hrs & $\begin{array}{l}80 \times 40,1920 \\
40 \times 40,960\end{array}$ & 1-3 days & $240-790$ \\
\hline $\begin{array}{l}\text { OMI } \\
(2004-)\end{array}$ & 13:30 hrs & $\begin{array}{l}13 \times 24 \\
2600\end{array}$ & 1 day & $270-500$ \\
\hline $\begin{array}{l}\text { TROPOMI } \\
(2017-)\end{array}$ & 13:30 hrs & $\begin{array}{l}7 \times 3.5 \\
2600\end{array}$ & 1 day & $\begin{array}{l}270-500 \\
675-775 \\
2305-2385\end{array}$ \\
\hline
\end{tabular}

${ }^{*}$ At nadir

All these satellites measure backscattered solar radiation in the ultraviolet and visible (UV-Vis) spectral range relevant for $\mathrm{NO}_{2}$. GOME, SCIAMACHY, GOME-2 and TROPOMI also measure in the near-infrared (NIR) around $760 \mathrm{~nm}$, which allows also to obtain cloud information from the oxygen $\left(\mathrm{O}_{2}\right)$ A-band. For OMI, the $\mathrm{O}_{2}-\mathrm{O}_{2}$ absorption band is used to retrieve clouds. SCIAMACHY and TROPOMI also measure in the shortwave infrared spectral band (SWIR, 2305 - $2385 \mathrm{~nm}$ ), from which key atmospheric species to monitor air quality and climate like methane and carbon monoxide can be retrieved. OMI and TROPOMI are afternoon sensors (i.e. local Equator crossing time is around 13:30 local time (LT))(see Table 2.1), and the others are morning sensors (local Equator crossing time around $10 \mathrm{LT}$ ). The combination of $\mathrm{NO}_{2}$ measurements from morning and afternoon sensors has made it possible to study the diurnal cycle of $\mathrm{NO}_{2}$ (e.g. using SCIAMACHY and OMI in Boersma et al. (2008)). One of the main differences between all the sensors is the horizontal resolution and the width of the swath, which mostly determines the spatial coverage. For GOME and GOME-2, daily coverage is achieved at low and mid latitudes for measurements done at coarser resolution. Because OMI and TROPOMI have a wider swath, they provide daily global coverage. TROPOMI has set new standards for remote sensing of atmospheric composition by providing measurements at an unprecedented spatial resolution of $7 \times 3.5 \mathrm{~km}^{2}$. 
The state-of-the-art retrievals that are currently applied to these four sensors do not produce consistent $\mathrm{NO}_{2}$ data due to intrinsic differences in the retrieval algorithms. When applying different retrievals to the same satellite observations, even though the retrievals are based on the same general approach, the outcome can be substantially different (Van Noije et al., 2006). The different algorithms developed by different retrieval groups have all their strengths and weaknesses, so the challenge in QA4ECV was to bring the strengths together to create an improved retrieval algorithm. In this chapter we give an overview of the main achievements within the QA4ECV project in the process of producing a coherent and improved retrieval algorithm that has been applied to the four sensors discussed above. First we describe the three retrieval steps and the improvements that we have implemented for each step. Then we give an overview of the QA4ECV $\mathrm{NO}_{2}$ 1995-2017 data record and finally we present some preliminary validation results that show the better agreement of the QA4ECV NO 2 product with independent ground-based measurements.

\section{2 $\mathrm{NO}_{2} \mathrm{QA4ECV}$ retrieval algorithm}

The retrieval of the tropospheric $\mathrm{NO}_{2}$ vertical column density from the measured radiance spectra by the satellite instrument consists of three steps:

1. A spectral fitting of a modelled reflectance spectrum to the measured radiance spectrum to obtain the amount of $\mathrm{NO}_{2}$ along the average photon path between the Sun and the satellite, i.e. the total slant column density $\left(N_{\mathrm{s}}\right.$, SCD) (Fig. 2.1 (a)).

2. The estimation of the stratospheric $\mathrm{NO}_{2}$ contribution to the total slant column density $\left(N_{\mathrm{s}, \text { strat }}\right)$ to obtain the tropospheric slant column density (Fig. 2.1 (b)).

3. The calculation of the air mass factor $(M, \mathrm{AMF})$ with a radiative transfer model (RTM) to convert the slant column density to a vertical column density (Fig. 2.1 (c)).

The retrieval equation is then written as:

$$
\hat{x}_{\mathrm{tr}}=\frac{N_{\mathrm{s}}(\vec{y})-N_{\mathrm{s}, \mathrm{strat}}}{M_{\mathrm{tr}}\left(x_{\mathrm{a}, \mathrm{tr}}, \overrightarrow{\mathbf{b}}\right)}
$$

Where $\vec{y}$ is the measured radiance spectrum from which the slant column density $N_{\mathrm{s}}$ is obtained, $M_{\mathrm{tr}}$ is the tropospheric air mass factor that depends on the a priori profile in the troposphere $x_{\mathrm{a}, \mathrm{tr}}$ and $\vec{b}$ the forward model (i.e. radiative transfer model) parameters describing the state of the atmosphere (i.e. surface albedo, surface altitude and clouds). 

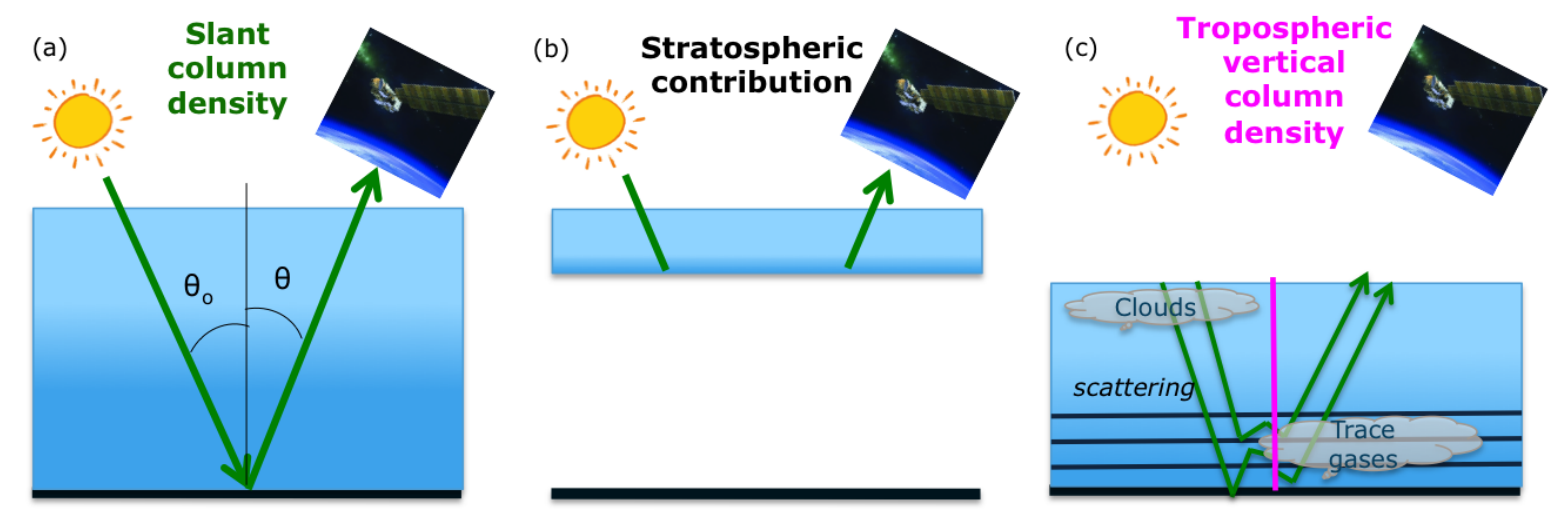

Figure 2.1: Sketch of the three steps in the $\mathrm{NO}_{2}$ retrieval algorithm explained in Section 2.2. Green line in (a) and (b) represent the geometrical path of the direct light from the sun to the surface and back to the satellite, without interacting with the atmosphere. Green line in (c) sketches the modification of the light path due to different processes in the atmosphere. Pink line in (c) is the vertical column for which the tropospheric vertical column density is retrieved from the satellite.

To develop the QA4ECV algorithm, a systematic analysis of the three retrieval steps was performed, comparing different approaches by the different retrieval groups within the project consortium, formed by retrieval experts from BIRA-IASB, IUP Bremen, KNMI, Max Planck Institute for Chemistry, and Wageningen University. All the approaches analysed in QA4ECV are part of well-established retrieval algorithms, so in order to decide which approach to adopt we had to perform dedicated inter-comparison experiments and discuss the scientific relevance in multiple meetings throughout the algorithm development phase. All these efforts pointed to the procedures that could be improved and lead us to create a "community best practice" retrieval algorithm that can be applied consistently to four different sensors using the same methodology and assumptions.

In the subsections below we describe each of the retrieval steps and the improvements made in QA4ECV with respect to previous state-of-the-art retrieval algorithms (e.g. DOMINO v2 (Boersma et al., 2011)). Following the QA4ECV philosophy, Zara et al. (2018) compared the slant column densities from the retrieval step 1 computed by different retrieval groups including QA4ECV, with special emphasis on the characterization of the uncertainties of the SCDs. Within QA4ECV, different stratospheric correction approaches were investigated in detail (Beirle, 2016) and are summarized in Sect. 2.2.2. Finally, because the air mass factor is a significant source of uncertainty in the retrieval process, it is of central importance in this chapter (Sect. 2.2.3) and in Chapter 3. 


\subsubsection{Slant column density}

The first step of the retrieval uses the Differential Optical Absorption Spectroscopy (DOAS) method (Platt, 2017) to obtain the slant column density, which is the number of $\mathrm{NO}_{2}$ molecules along the average light path. The DOAS method is based on the Beer-Lambert Law that describes the attenuation of light passing through a medium (here the atmosphere). A modelled reflectance spectrum is fitted to the observed reflectance by the satellite using a least squares technique that minimizes the differences. The modelled spectrum describes the effects of absorption by molecules, the effects of inelastic rotational Raman scattering (i.e. Ring effect) and includes a polynomial that accounts for spectrally smooth signals (e.g. from Rayleigh and Mie scattering, spectral surface reflection and possible instrumental effects):

$$
I(\lambda)=I_{0}(\lambda) \exp \left[\left(-\sum_{\mathrm{i}=1}^{\mathrm{N}} \sigma_{\mathrm{i}}(\lambda) \mathrm{N}_{\mathrm{s}, \mathrm{i}}-\sum_{\mathrm{P}} \mathrm{a}_{\mathrm{P}} \lambda^{\mathrm{P}}\right)\right]
$$

This equation can also be written as a function of the optical depth:

$$
\ln \left[\frac{I(\lambda)}{I_{0}(\lambda)}\right]=\sum_{i=1}^{\mathrm{N}} \sigma_{i}(\lambda) N_{s, i}+\sum_{\mathrm{P}} \mathrm{a}_{\mathrm{P}} \lambda^{\mathrm{P}}
$$

In both Eqs. 2.2 and 2.3, $I(\lambda)$ is the radiance, $I_{0}(\lambda)$ the solar irradiance or reference spectrum, $\sigma_{i}(\lambda)$ the absorption cross section of the absorber i, $N_{s, i}$ the slant column density and $a_{\mathrm{P}}$ the coefficients of the polynomial. Any of the equations above can be used in the fit; using Eq. 2.2 is the so-called "intensity fit" and using Eq. 2.3 is the "optical density fit". The advantage of the optical density fit is that it is a linear fit with respect to the quantity of interest, any slant column density.

In order to establish the optimal settings for the spectral fitting within QA4ECV, an extensive comparison of fitting approaches and their uncertainties from different retrievals codes was performed for OMI and GOME-2A (Müller et al., 2016). For GOME-2 (and also SCIAMACHY and GOME), the standard wavelength interval that is used in state-of-theart retrievals to perform the $\mathrm{NO}_{2}$ spectral fitting is $425-450 \mathrm{~nm}$ (e.g. Valks et al. (2011), Boersma et al. (2004)). For OMI, in order to improve the signal-to-noise ratio, the fitting window was extended to 405-465 $\mathrm{nm}$ in the DOMINO retrieval (Boersma et al., 2002), and this is the window that is currently used for TROPOMI (van Geffen et al., 2018). Table 2.2 gives an overview of the main settings for the $\mathrm{NO}_{2}$ spectral fitting from different retrieval algorithms for OMI (QA4ECV and OMNO2A v2) and GOME-2A (QA4ECV and TM4NO2A v2.3). In QA4ECV, the fitting window for GOME, SCIAMACHY and GOME2 was extended from $425-450 \mathrm{~nm}$ to $425-465 \mathrm{~nm}$ to improve the consistency with the OMI 
405-465 nm window. Because of a better agreement between the different retrievals, it was decided to use an optical density fit. The choice of the intensity fit or the optical density fit leads to systematic bias up to $1 \cdot 10^{15} \mathrm{molec} \cdot \mathrm{cm}^{-2}$, therefore the same type of fit was applied to all four sensors (Boersma et al., 2018).

In the QA4ECV slant column inter-comparison, whether to include an intensity offset correction was discussed and dedicated experiments were performed to investigate its relevance and to understand its meaning. This intensity offset might account for instrumental effects, e.g. straylight inside the spectrometer and dark current (Peters et al., 2014). The intensity offset can also compensate for spectral features caused by inelastic vibrational Raman scattering (VRS) on liquid water molecules, which is most significant for measurements over cloud-free ocean scenes (e.g. Peters et al. (2016), Richter et al. (2011)). If the intensity offset is not included, the Ring effect correction might partly account for these VRS effects. Figure 2.2 shows that the fitted intensity offset coefficients are positive and correlate well with clear-sky chlorophyll-free ocean scenes, as well as with the liquid water fit coefficients (Fig. 2.3) strongly suggesting that this term indeed represents vibrational Raman scattering in open water. Including this term results in better fit results, with a $10 \%$ reduction in the RMS of the fit over cloud-free ocean scenes and $9 \%$ smaller SCD uncertainties (Oldeman, 2018). Over cloud-free ocean scenes, a positive intensity offset results in differences in $\mathrm{NO}_{2}$ SCDs up to $15 \%$, with mean differences of $4 \%$ (Oldeman, 2018). Although most of the pollution sources are over land where the intensity offset is negligible, the SCDs over ocean scenes are relevant for the stratospheric estimation in the second step of the retrieval, so the intensity offset should be included. In the QA4ECV retrieval, a correction to account for an intensity offset is included in the fitting model with a polynomial $\left(P_{\text {off }}(\lambda)\right.$ in Eq. 2.4).

The modelled reflectance used in the QA4ECV optical density fit is based on Eq. 2.3, with an extra term $P_{\text {off }}(\lambda)$ to account for the intensity offset:

$$
R_{\mathrm{mod}}=\ln \left[\frac{I(\lambda)-P_{\mathrm{off}}(\lambda)}{I_{0}(\lambda)}\right]=P(\lambda)-\sum_{i=1}^{\mathrm{N}} \sigma_{i}(\lambda) N_{s, i}-\sigma_{\text {Ring }}(\lambda) C_{\text {Ring }}+r(\lambda)
$$

where $P(\lambda)$ is the DOAS polynomial, and $\sigma_{\text {Ring }}$ is the cross-section of the Ring effect, which is accounted for by introducing a pseudo-absorber. In the fitting procedure, a $\chi^{2}$-minimisation of the residual $r(\lambda)$ is performed (Zara et al., 2018) using the QDOAS software for OMI and GOME-2A (2012-2017) (Danckaert et al., 2017) and NLIN (Richter, 1997) for SCIAMACHY, GOME and GOME-2A (2007-2011).

The comparison of approaches led to improvements in the spectral algorithm, mainly through including liquid water absorption and the intensity offset correction. Overall, 
Table 2.2: Main settings of $\mathrm{NO}_{2}$ spectral fitting for TROPOMI, OMI (QA4ECV and OMNO2A v2) and GOME-2A (QA4ECV and TM4NO2A v2.3).

\begin{tabular}{|c|c|c|c|}
\hline & $\begin{array}{l}\text { Fitting } \\
\text { window (nm) }\end{array}$ & $\begin{array}{l}\text { Fitting } \\
\text { method }\end{array}$ & $\begin{array}{l}\text { Fitted } \\
\text { parameters }\end{array}$ \\
\hline TROPOMI $^{1}$ & $405-465$ & Intensity fit & $\begin{array}{l}\mathrm{O}_{3}, \mathrm{H}_{2} \mathrm{O}_{\text {vap }} \\
\mathrm{O}_{2}-\mathrm{O}_{2}, \mathrm{H}_{2} \mathrm{O}_{\text {liq }}\end{array}$ \\
\hline QA4ECV OMI ${ }^{2}$ & $405-465$ & $\begin{array}{l}\text { Optical } \\
\text { density fit }\end{array}$ & $\begin{array}{l}\mathrm{O}_{3}, \mathrm{H}_{2} \mathrm{O}_{\text {vap }}, \\
\mathrm{O}_{2}-\mathrm{O}_{2}, \mathrm{H}_{2} \mathrm{O}_{\text {liq }} \\
P_{\text {off }}\end{array}$ \\
\hline OMNO2A v2 $2^{3}$ & $405-465$ & Intensity fit & $\begin{array}{l}\mathrm{O}_{3}, \mathrm{H}_{2} \mathrm{O}_{\text {vap }} \\
\mathrm{O}_{2}-\mathrm{O}_{2}, \mathrm{H}_{2} \mathrm{O}_{\text {liq }}\end{array}$ \\
\hline QA4ECV GOME-2A ${ }^{2}$ & $405-465$ & $\begin{array}{l}\text { Optical } \\
\text { density fit }\end{array}$ & $\begin{array}{l}\mathrm{O}_{3}, \mathrm{H}_{2} \mathrm{O}_{\text {vap }}, \\
\mathrm{O}_{2}-\mathrm{O}_{2}, \mathrm{H}_{2} \mathrm{O}_{\text {liq }} \\
P_{\text {off }}\end{array}$ \\
\hline TM4NO2A v2.3 & $425-450$ & $\begin{array}{l}\text { Optical } \\
\text { density fit }\end{array}$ & $\begin{array}{l}\mathrm{O}_{3}, \mathrm{H}_{2} \mathrm{O}_{\text {vap }} \\
\mathrm{O}_{2}-\mathrm{O}_{2}, P_{\text {off }}\end{array}$ \\
\hline
\end{tabular}

(1) van Geffen et al. (2018), (2) Boersma et al. (2018), (3) van Geffen et al. (2015)

QA4ECV applies a more physically accurate fitting model to estimate the SCDs. The SCD uncertainties from QA4ECV are lower compared to state-of-the-art retrieval and these uncertainties are free of viewing or solar angle dependencies (Zara et al., 2018). For the OMI sensor the mission-average SCDs uncertainties for QA4ECV are $0.84 \cdot 10^{15}$ molec $\cdot \mathrm{cm}^{-2}$ and $0.99 \cdot 10^{15}$ molec $\cdot \mathrm{cm}^{-2}$ for OMNO2A v2 (DOMINO v2), which is a $16 \%$ improvement. For GOME-2A, the main difference between QA4ECV and the current operational fit in the TM4NO2A (v2.3) retrieval is that QA4ECV uses a larger fitting window and includes liquid water absorption. The SCD uncertainties for GOME-2A are also lower in the QA4ECV retrieval by $11 \%\left(0.80 \cdot 10^{15}\right.$ molec $\cdot \mathrm{cm}^{-2}$ vs. $0.89 \cdot 10^{15}$ molec $\cdot \mathrm{cm}^{-2}, 2005-2015$ average).

The spectral fitting in the TROPOMI $\mathrm{NO}_{2}$ retrieval algorithm incorporates most of the developments from QA4ECV for the DOAS fit (van Geffen et al., 2018). However, two important lessons have not been adopted yet. The fitting method is the intensity fit (Eq. 2.2), and it was chosen because it allows to explicitly account for the Ring effect (van Geffen et al., 2015). In contrast to what was decided in QA4ECV, the intensity offset was not included in the first version of the retrieval algorithm, and the importance of this correction on real data is currently being investigated.

The optimization of spectral fitting methods that has been ongoing in the last years for $\mathrm{NO}_{2}$ satellite measurements (e.g. inclusion of liquid water, vibrational raman scattering in water)(van Geffen et al. (2015), Boersma et al. (2018)) together with the much improved signal-to-noise ratio and precision of TROPOMI (van Geffen et al., 2018), has led to substantial improvement in the $\mathrm{NO}_{2}$ slant column densities (i.e. SCD uncertainties are 

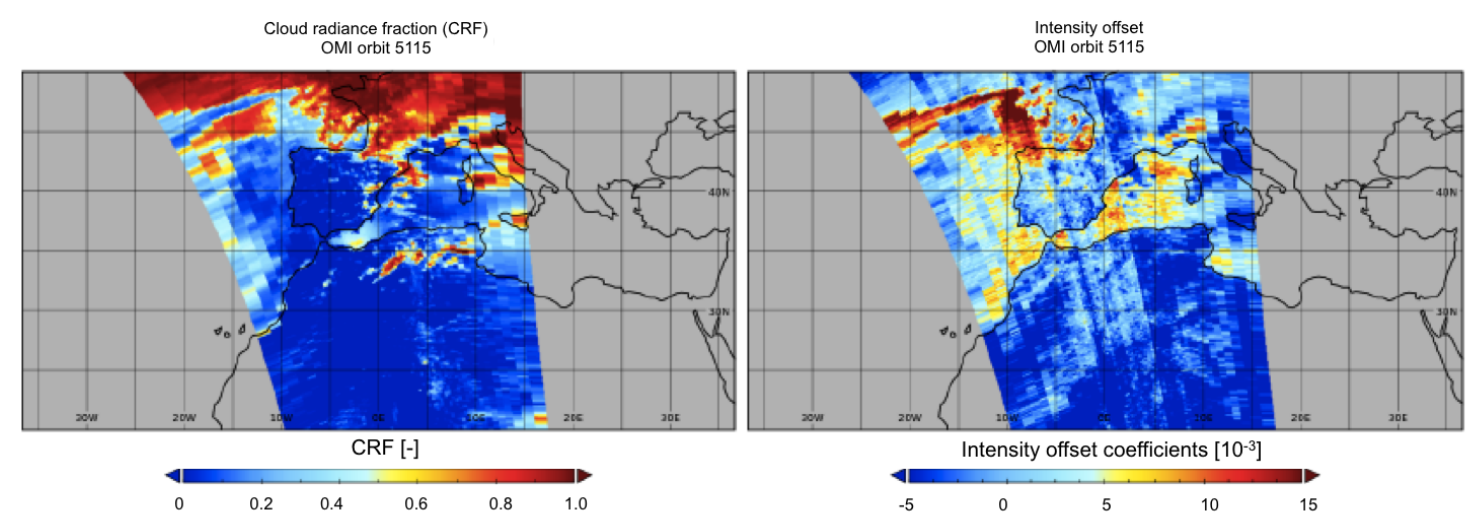

Figure 2.2: (a) OMI cloud radiance fraction and (b) intensity offset for part of the orbit 5115 over the Mediterranean Sea and Sahara (adapted from Oldeman (2018)).

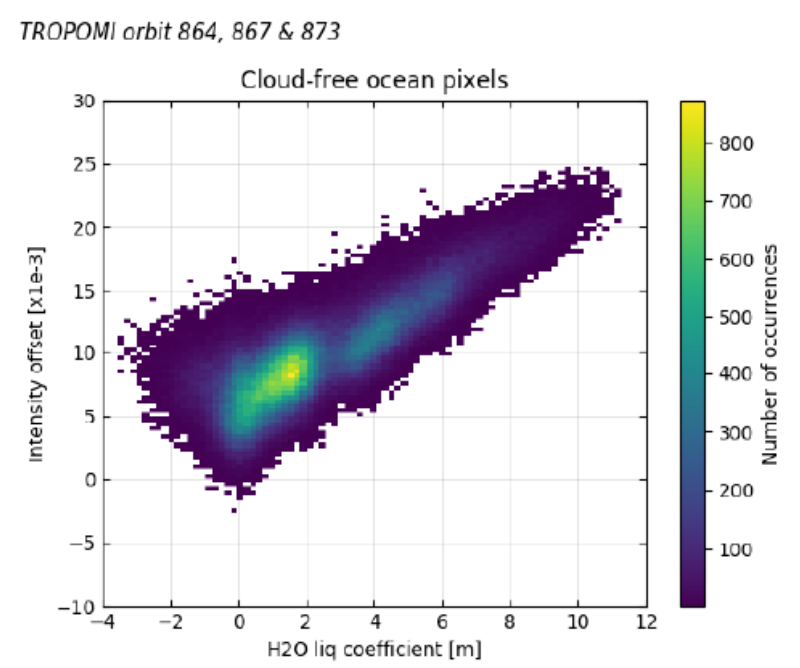

Figure 2.3: Intensity offset as a function of the liquid water coefficients for cloud-free ocean pixels for three TROPOMI orbits (0864, 0867 and 0873) (adapted from Oldeman (2018)).

lower than for OMI by a factor of $\left.2\left(0.5-0.610^{15} \mathrm{molec} \cdot \mathrm{cm}^{-2}\right)\right)$. As a consequence, we may anticipate to the possibility to detect weaker sources which were missed by the coarser OMI and GOME-2 pixels. This is also relevant to use TROPOMI $\mathrm{NO}_{2}$ measurements to zoom in at urban scales and see enhancements of pollution over cities, as we will see in chapter 5 .

\subsubsection{Stratospheric $\mathrm{NO}_{2}$ column estimation}

$\mathrm{NO}_{2}$ satellite measurements do not provide information on the vertical distribution of $\mathrm{NO}_{2}$, and the retrieved slant column density also contains contributions from stratospheric 
$\mathrm{NO}_{2} . \mathrm{NO}_{2}$ concentrations in the stratosphere are not negligible, and its source is mainly from the oxidation of $\mathrm{N}_{2} \mathrm{O}$ to form $\mathrm{NO}_{x}$ (Cohen and Murphy, 2003). In order to determine the tropospheric $\mathrm{NO}_{2}$ column from the satellite measurements, it is necessary to accurately estimate the stratospheric contribution $\left(N_{s, \text { strat }}\right)$ to the total slant column density (see retrieval Eq. 2.1).

Within QA4ECV, three different stratospheric correction approaches were compared to establish the best method and assess the uncertainties for this retrieval step. The three methods are based on assumptions on the spatial distribution of stratospheric $\mathrm{NO}_{2}$. They use SCDs retrieved in clean remote regions where the contribution of tropospheric $\mathrm{NO}_{2}$ is small because of its short lifetime. Two of the methods use a chemistry transport model to simulate stratospheric $\mathrm{NO}_{2}$. IUP-Bremen scales the simulated VCDs with the B3dCTM model to match satellite observations over the remote Pacific (Hilboll et al., 2013). KNMI assimilates measured $\mathrm{NO}_{2} \mathrm{SCDs}$ in the TM4 model so the simulated stratospheric $\mathrm{NO}_{2}$ fields are in close agreement with satellite observations over remote regions (Dirksen et al., 2011). MPI-C applies the STREAM algorithm to satellite observations to estimate stratospheric VCDs over remote regions and also over clouded scenes where tropospheric $\mathrm{NO}_{2}$ is shielded (Beirle et al., 2016). The contribution of the stratosphere to the total $\mathrm{NO}_{2}$ from individual measurements is estimated using weighting factors that account for possible pollution and cloudy observations. Global daily maps of stratospheric $\mathrm{NO}_{2}$ are provided using weighted convolution without the need of using a chemistry transport model.

The three different approaches showed very good agreement for the stratospheric columns and the tropospheric residue $\left(N_{s, \text { trop }}=N_{s}-N_{s, \text { strat }}\right)$. Differences for two individual days and months were smaller than $0.510^{15} \mathrm{molec} \cdot \mathrm{cm}^{-2}$ (around $15 \%$ ) (Müller et al., 2016). For the QA4ECV algorithm, it was decided to use the data assimilation approach by KNMI, with several developments with respect to the current version used in DOMINO v2. The use of this approach makes QA4ECV retrieval algorithm more consistent, as $\mathrm{NO}_{2}$ a priori profiles used for the air mass factor calculation are estimated using the same CTM (TM5-MP) as in the data assimilation scheme. A major improvement of the data assimilation approach used in QA4ECV is the use of the TM5-MP instead of the TM4 version. This implies a better spatial resolution $\left(1^{\circ} \times 1^{\circ}\right.$ instead of $\left.3^{\circ} \times 2^{\circ}\right)$, updated reaction rates for the relevant chemical processes, an improved parametrization of photolysis rate constants and year specific emission inventories until 2010 (Williams et al., 2017). All these developments in the data assimilation resulted in about $15 \%$ less negative tropospheric values at high latitudes and over oceans, a problem that was present in the DOMINO retrieval (Maasakkers, 2013). Maasakkers (2013) also reported that negative columns often occurred for large solar zenith angles (SZA), suggesting a systematic error in the AMFs. From the detailed radiative transfer model inter-comparison done in 
QA4ECV, we could attribute this to the lack of a full sphericity treatment in the radiative transfer (Lorente et al., 2017). Therefore we included in the data assimilation a correction for sphericity using the McArtim radiative transfer model. This sphericity correction is further discussed in the next section and is of central importance in chapter 3.

The results for the stratospheric $\mathrm{NO}_{2}$ column by the STREAM algorithm are also included in the QA4ECV $\mathrm{NO}_{2}$ product, to give users the possibility to switch between approaches. Data assimilation (DA) resolves better the patterns caused by stratospheric dynamics and strong stratospheric $\mathrm{NO}_{2}$ gradients, while STREAM is more suitable when estimating $\mathrm{NO}_{x}$ emissions from weaker sources (e.g. ships). Figure 2.4 shows $\mathrm{NO}_{2}$ stratospheric fields averaged in the $39^{\circ}-41^{\circ}$ latitude range as a function of longitude computed by the DA system and by STREAM for one day of OMI measurements. The DA approach shows more variability within a zonal band, while STREAM shows more smooth stratospheric fields. This is because in STREAM algorithm the $\mathrm{NO}_{2}$ stratospheric fields are assumed to vary smoothly with longitude (Beirle et al., 2016). Between $75^{\circ}-125^{\circ}$ $\mathrm{W}$ (United States) and $0-40^{\circ} \mathrm{E}$ (Europe), DA stratospheric columns values are 0.2-0.5 $\cdot 10^{15}$ molec $\cdot \mathrm{cm}^{-2}$ lower than STREAM values. Over eastern Asia $\left(100^{\circ}-140^{\circ} \mathrm{E}\right), \mathrm{DA}$ and STREAM agree to within $0.3 \cdot 10^{15}$ molec $\cdot \mathrm{cm}^{-2}$. These differences between the two methods can be considered as the structural uncertainty in the stratospheric $\mathrm{NO}_{2}$ estimation.

The data assimilation for the stratospheric estimation with the two main improvements (the higher resolution CTM and the sphericity correction in the radiative transfer model) gives the QA4ECV algorithm consistency with respect to steps 2 and 3 in the retrieval. The new implementation results in fewer unphysical negative $\mathrm{NO}_{2}$ columns. Although this points in the right direction, further testing of the stratospheric fields with independent ground-based observations is necessary, both for the new data assimilation scheme and STREAM.

\subsubsection{Air mass factor calculation}

In the last step of the retrieval, an air mass factor (AMF, $M$ ) is calculated to convert the tropospheric slant column density into a vertical column density $\left(M=N_{\mathrm{s}} / N_{\mathrm{v}}\right)$. To calculate the AMF, a radiative transfer model is used together with prior knowledge on the atmospheric state. At each atmospheric layer $l$ the value of the AMF $m_{l}$ is computed (i.e. altitude-dependent AMF or box-AMF) and these are typically stored in the form of a look-up table (LUT). It is also possible to perform pixel-by-pixel online radiative transfer simulations to calculate the box-AMFs (e.g. Lin et al. (2014)) but this approach is computationally expensive for global retrievals. Furthermore, the LUT interpolation errors are negligible compared to other errors in the AMF calculation (Lorente et al., 


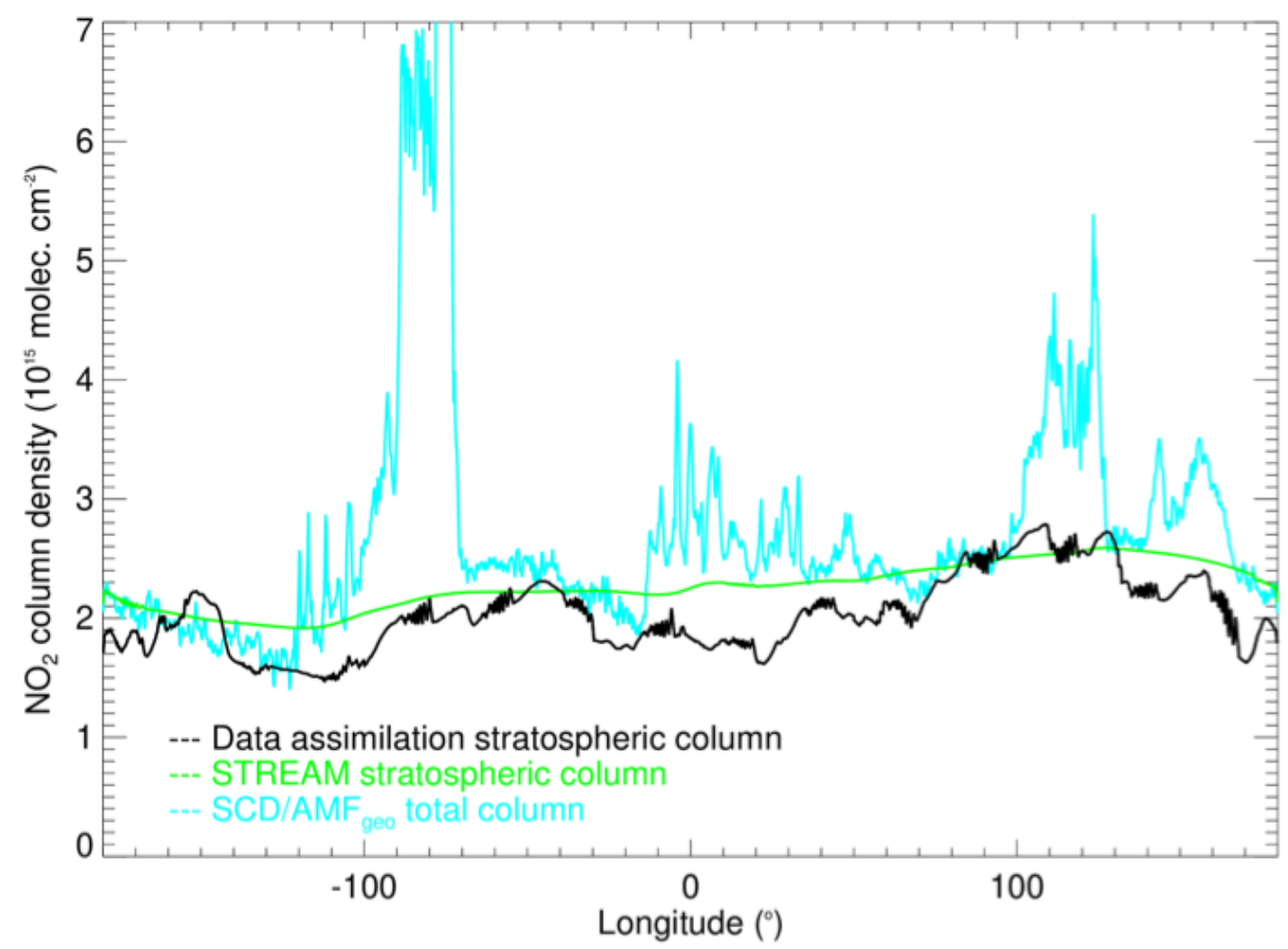

Figure 2.4: Data assimilation (black) and STREAM (green) stratospheric column meridional average over $39^{\circ}-41^{\circ} \mathrm{N}$ on 2 February 2005. QA4ECV OMI NO $\mathrm{N}_{2}$ total slant column divided by the geometric AMF is shown in light blue. No filter for cloud radiance, albedo, nor AMF has been applied (from Boersma et al. (2018)).

2017).

The dimensions of the LUT are associated with the knowledge of the atmospheric state and are the a priori parameters of the forward model $(\widehat{\mathbf{b}})$ such as satellite viewing geometry, surface pressure, surface reflectivity, cloud fraction and cloud pressure. The a priori $\mathrm{NO}_{2}$ profile $\left(x_{a, l}\right)$ from a CTM (e.g. TM5-MP in QA4ECV) is used for the application of the LUT to calculate total, stratospheric, and tropospheric AMFs:

$$
M=\frac{\sum_{l} m_{l}(\widehat{\mathbf{b}}) x_{a, l}}{\sum_{l} x_{a, l}}
$$

The sum in Eq. 2.5 over the atmospheric layers $l$ of $x_{a, l}$ represents the vertical column $N_{\mathrm{v}}$, and the sum of $m_{l}(\widehat{\mathbf{b}}) x_{a, l}$ represents the slant column $N_{\mathrm{s}}$. The $\mathrm{NO}_{2}$ absorption cross sections are representative of one fixed temperature. However, these cross sections vary with temperature, and commonly a correction factor is applied for each layer in the AMF calculation based on temperature and trace gas profiles from model data or climatologies. 
Not accounting for this variation in the temperature can lead to systematic errors of around 20\% (Boersma et al., 2004).

Satellite retrievals also need to account for the presence of clouds, and that is done in the AMF calculation. There are several cloud corrections commonly used to account for residual clouds. The independent pixel approximation (IPA) consists of calculating the AMF for a partly cloudy scene as a linear combination of cloudy $\left(M_{\mathrm{cl}}\right)$ and clear $\left(M_{\mathrm{cr}}\right)$ components of the AMF, weighted by the fraction of radiance that originates from the cloudy part of the pixel. The atmosphere can also be assumed to be cloud-free for cloud fractions below a certain threshold and in that case, a clear-sky AMF is used. These different cloud correction approaches are discussed in detail in Chapter 3.

Within QA4ECV, a very extensive and detailed comparison of AMF calculation approaches was performed to evaluate the method to use in QA4ECV and to investigate in detail the uncertainties in this step of the retrieval. This comparison exercise was extended to other groups outside the QA4ECV consortium (University of Leicester, NASA-GSFC and Peking University), to ensure that a proper sample of the different approaches was represented. With this comparison we estimated the "structural uncertainty" in the air mass factor, which represents the uncertainty that arises due to the choice of the RTM and of the input parameters in every step of the calculation. The details of this comparison can be found in Chapter 3, and in the remainder on this section we discuss some other important aspects of the calculation of the AMFs for QA4ECV and TROPOMI.

After the extensive comparison, we decided to create the QA4ECV AMF $\mathrm{NO}_{2}$ LUT with the radiative transfer model DAK. To calculate the AMFs, first we simulate topof-atmosphere reflectance $(R)$ with and without $\mathrm{NO}_{2}$ in a particular atmospheric layer $l$, and then compute the altitude-dependent (or box-) air mass factor $\left(m_{l}\right)$ by differencing the logarithm of the reflectance divided by the absorption optical thickness of the layer with $\mathrm{NO}_{2}, \tau_{\text {gas }, l}$. Afterwards Eq. 2.5 is applied to obtain the tropospheric AMF.

$$
m_{l}=-\frac{\ln R\left(\tau_{\mathrm{gas}, l}\right)-\ln R\left(\tau_{\mathrm{gas}, l}=0\right)}{\tau_{\text {gas }, l}}
$$

DAK radiative transfer model (Stammes et al. (1989), Lorente et al. (2017)) has been used for high-quality satellite retrieval applications, such as trace gas, cloud and aerosol retrievals (e.g. Boersma et al. (2011), Wang et al. (2008), Chimot et al. (2016)). DAK solves the radiative transfer equation using the doubling adding method. The method consists of first calculating the reflection and transmission properties of a homogeneous layer by repeated doubling, starting with a very thin layer, and then adding homogeneous layers on top of each other, which then yields the reflection and transmission of the combined layers (de Haan et al., 1987). 


\section{Polarization}

Inclusion of polarization is important in radiative transfer calculations and for satellite retrievals in the UV/Vis (Lacis et al., 1998), thus we investigated the effect of polarization in DAK. Neglecting polarization can lead to errors in the simulated TOA reflectance (and therefore in the AMF), although these errors are relatively small if a large contribution of the light is from reflection on aerosols or larger particles instead of mainly from molecular multiple scattering (Lenoble, 1993). Including polarization may change the reflectance with a strong dependence on both solar and viewing zenith angles.

The simulations with DAK can be set up to neglect polarization, in which case the Stokes vector ${ }^{1}$ is one dimensional and reflectance simulations are referred to as scalar. DAK can also account for polarization, in which case all the components of the Stokes vector are computed and the reflectance simulations are referred to as vector reflectance. We performed scalar and vector TOA reflectance simulations using DAK to estimate the effect of neglecting polarization in our RTM simulations. The experiment was performed at $440 \mathrm{~nm}$ for a clean mid-latitude summer standard atmosphere (Anderson et al., 1986), without aerosols and for a surface albedo of 0.05 .

Figure 2.5 shows relative differences of scalar and vector TOA reflectance. Scalar and vector TOA reflectances differ up to $7 \%$ in nadir view, with under or overestimation depending on the solar and viewing geometry. For viewing geometries typically ocurring in mid-latitudes (e.g. equal viewing and solar zenith angle of $45^{\circ}$, orange lines in Fig. 2.5 ) vector TOA reflectances are $2-4 \%$ higher than the scalar simulations. Including polarization is a more realistic description of the vector nature of light, therefore we compute all the radiative transfer simulations (i.e. TOA reflectances) with polarization for the calculation of the AMFs for QA4ECV and TROPOMI $\mathrm{NO}_{2}$ retrievals. This was also the choice for the RTM and AMF comparison in Chapter 3.

\section{Wavelength selection}

The air mass factor determines the average light path of photons travelling from the sun through the atmosphere and back to the satellite (Fig. 2.7c). Some processes that affect the light path through the atmosphere depend on wavelength (e.g. Rayleigh scattering). At shorter wavelengths, due to stronger Rayleigh scattering, light paths are attenuated more strongly than at longer wavelengths. The absorption properties of the specific trace gas also depend on wavelength, and $\mathrm{NO}_{2}$ has strong absorption properties around 440 $\mathrm{nm}$, as shown by the $\mathrm{NO}_{2}$ absorption cross-section on the right axis in Fig. 2.6.

\footnotetext{
${ }^{1}$ Stokes vector or Stokes parameters $(I, U, Q, V)$ describe the polarization state of light propagating through a medium.
} 


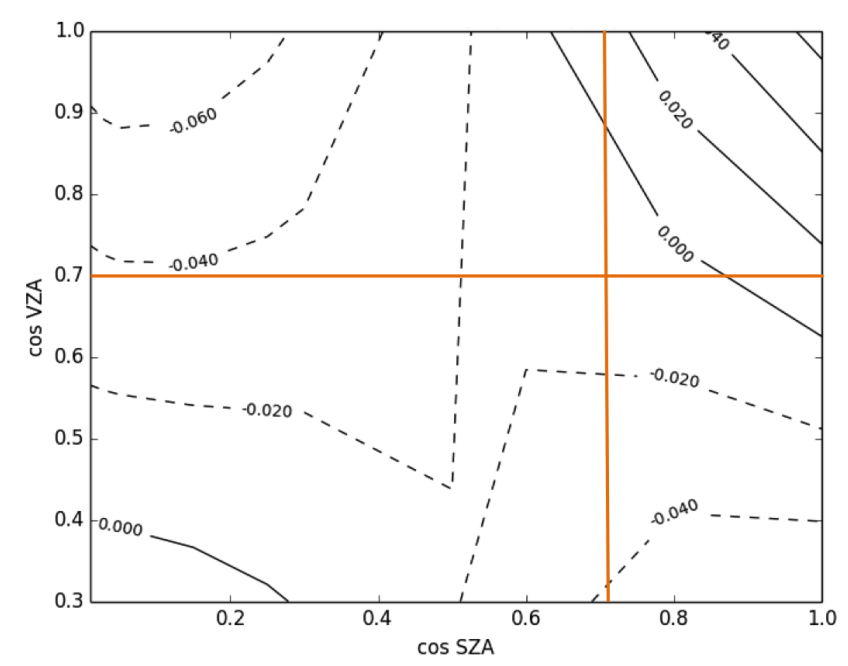

Figure 2.5: Contour lines of relative differences between scalar TOA reflectance and vector TOA reflectance as a function of cosine of viewing zenith angle (VZA) and cosine of solar zenith angle $(\mathrm{SZA})$ at $440 \mathrm{~nm}$. Orange lines represent typical mid-latitude viewing and solar angle of $45^{\circ}$. Simulations are shown for nadir view $\left(\phi-\phi^{\prime}=0^{\circ}\right)$ and surface albedo of 0.05 . A clean mid-latitude standard atmosphere is considered.

Slant column densities are retrieved in a spectral window that is several nm wide (e.g. 405-465 nm for OMI and TROPOMI $\mathrm{NO}_{2}$, Table 2.2), so it is important to establish the appropriate wavelength to calculate the $\mathrm{NO}_{2}$ AMFs. The tropospheric AMF calculated at one single wavelength should be representative for the fit-window average AMF. We investigated the tropospheric AMF wavelength dependency for retrieval scenarios with substantial tropospheric pollution in the boundary layer $\left(N_{v \text {,trop }}=1.6 \cdot 10^{16} \mathrm{molec} \cdot \mathrm{cm}^{-2}\right.$, around $7 \mathrm{ppb}$ ). We calculated AMFs using DAK for a mid-latitude standard atmosphere in steps of $1 \mathrm{~nm}$ from 405 to $500 \mathrm{~nm}$, the spectral range that covers the three fitting windows used within QA4ECV and by the individual groups (Table 2.2).

Figure 2.6 shows a close-to-linear tropospheric AMF increase with wavelength, reflecting the increasing transparency of the atmosphere towards the red part of the spectrum where Rayleigh scattering is relatively weak. The $\mathrm{NO}_{2}$ AMF increases by $0.22-0.33 \%$ per $\mathrm{nm}$ redshift to the visible. Horizontal blue, light blue and purple lines in Fig. 2.6 show the mean AMF calculated over all spectral points in the three frequently used fitting windows.

For the fitting windows considered for $\mathrm{NO}_{2}$ retrievals, blue and purple lines in Fig. 2.6 indicate that $437.5 \mathrm{~nm}$ is a representative wavelength to calculate the $\mathrm{NO}_{2} \mathrm{AMF}$. $437.5 \mathrm{~nm}$ is reasonably near to the centre wavelength of the fitting windows and the 437.5 $\mathrm{nm}$ AMF is within $2 \%$ of the window-average AMF for both windows. Given the need for a simple, unambiguous AMF approach for the entire QA4ECV record, we use the 


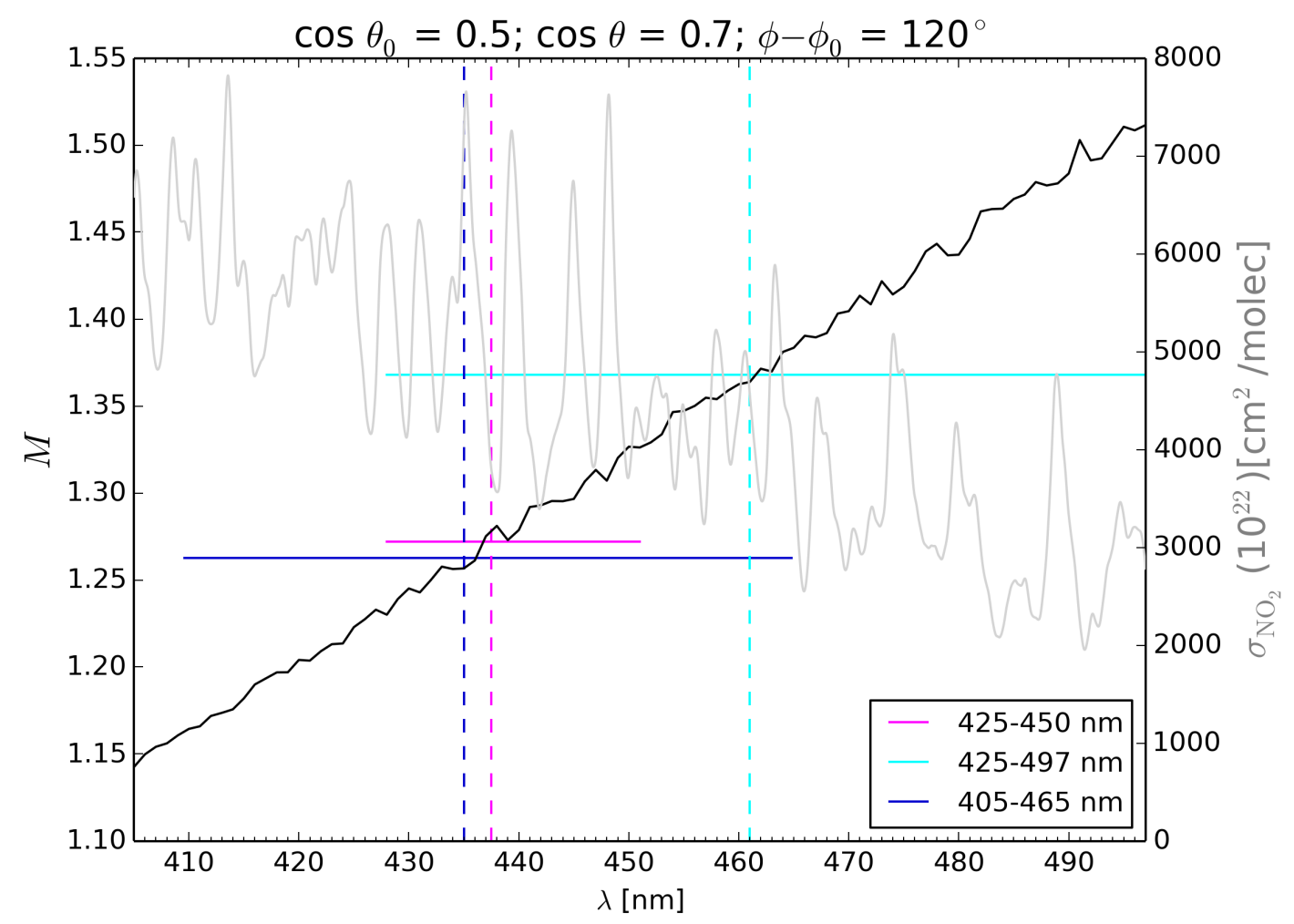

Figure 2.6: $\mathrm{NO}_{2}$ tropospheric air mass factor $M$ (black) as a function of wavelength computed with DAK for a polluted boundary layer for a specific viewing geometry $\left(\theta=60^{\circ}, \theta_{0}=45.6^{\circ}\right)$. Horizontal lines show averaged multi-wavelength AMF for different fitting windows (purple, 425-450 nm, blue 405-465 nm and light blue 425-497 nm). The grey line shows $\mathrm{NO}_{2}$ absorption cross-section from Vandaele et al. (1998) at $220 \mathrm{~K}$. A mid-latitude standard atmosphere was used including $\mathrm{O}_{3}$. The AMF was computed for a polluted boundary layer with $16 \times 10^{15}$ molec $\cdot \mathrm{cm}^{-2}$, without aerosols, a boundary layer height of $1 \mathrm{~km}$ and surface albedo 0.05 .

same $437.5 \mathrm{~nm}$ box AMF LUT for all sensors in QA4ECV. Uncertainties related to the exact choice of AMF wavelength calculation (around 2\%) are much smaller than AMF uncertainties due to other choices (around 40\%), such as clouds, albedo, trace gas and aerosol profiles, as discussed in Chapter 3.

The largest fitting window (425-497 nm) has been used in retrievals from GOME-2 (Richter et al., 2011) and in the TROPOMI Verification Retrieval (S5P Verification Team, 2015). The use of a wider and red-shifted fitting window improves the sensitivity to $\mathrm{NO}_{2}$ in the boundary layer, as the contribution from Rayleigh scattering in the atmosphere decreases. Figure 2.6 shows that in the large fitting window AMF varies up to $20 \%$ between the edges. In highly polluted scenes, the AMF wavelength dependency is detected in the slant column densities from the fit using the 425-497 nm window (S5P Verification Team, 2015). Because of a higher sensitivity, the SCDs are higher for fitting windows shifted to the blue and red parts of the spectrum when $\mathrm{NO}_{2}$ is close to the surface (Behrens et al., 2018). Therefore, when using a wider and red-shifted fitting window (for QA4ECV we 
use 405-465 nm) and especially for high polluted scenes, the AMF wavelength dependency should be reconsidered.

\section{Sphericity correction}

Radiative transfer models simulate the transport of radiation in a plane parallel approximation or accounting for Earth's sphericity. Different RTMs have different degrees of correction for Earth's sphericity. McArtim is a full 3D spherical model that simulates radiation fields in a spherical atmosphere. VLIDORT and SCIATRAN account for sphericity on solar incoming and single-scattered photons, but not for multiple-scattered ones. DAK in its pseudo spherical mode corrects the path for direct solar incident photons but not for the scattered ones.

Differences between TOA reflectances with the plane parallel and the pseudo spherical approximation in DAK become substantial (up to 22\%) when the solar zenith angle is large (i.e when the sun is low). For high solar zenith angles, McArtim AMFs are systematically lower than AMFs computed with the other RTMs, especially in the upper and free troposphere. The reason is that McArtim fully accounts for Earth's sphericity for both direct and scattered light. This means that light paths in McArtim are bounded by Earth's sphere, while in plane parallel mode photons can reach TOA from far away in the horizon, with longer light paths that result in higher AMFs (see Fig. 2.7). This is of particular importance for a correct estimation of the stratospheric contribution to the total slant column density in the second step of the retrieval. Too (incorrect) high stratospheric fields can lead to negative tropospheric columns.

In QA4ECV we use DAK RTM with a correction factor that accounts for sphericity based on McArtim. Retrieval results suggest that this correction factor has contributed to having fewer negative tropospheric columns in the QA4ECV retrieval, together with the improvements in the data assimilation approach. This is discussed in detail in Sect. 3.4 in Chapter 3.

\section{Reference points in the LUT}

The AMF LUT that contains the box-AMFs $\left(m_{l}\right.$, Eq. 2.6) is a 6-dimensional table. The dimensions correspond to the a priori parameters of the forward model (i.e. viewing geometry (x 3), surface albedo, surface pressure and altitude). For each combination of the parameters, a multidimensional interpolation is performed to compute the AMF. A proper density of reference points in the LUT is advantageous to minimize interpolation errors. To estimate the proper number of reference points in the LUT, we investigated the sensitivity of $\mathrm{NO}_{2}$ air mass factor to the input parameters. For low albedo values, the 

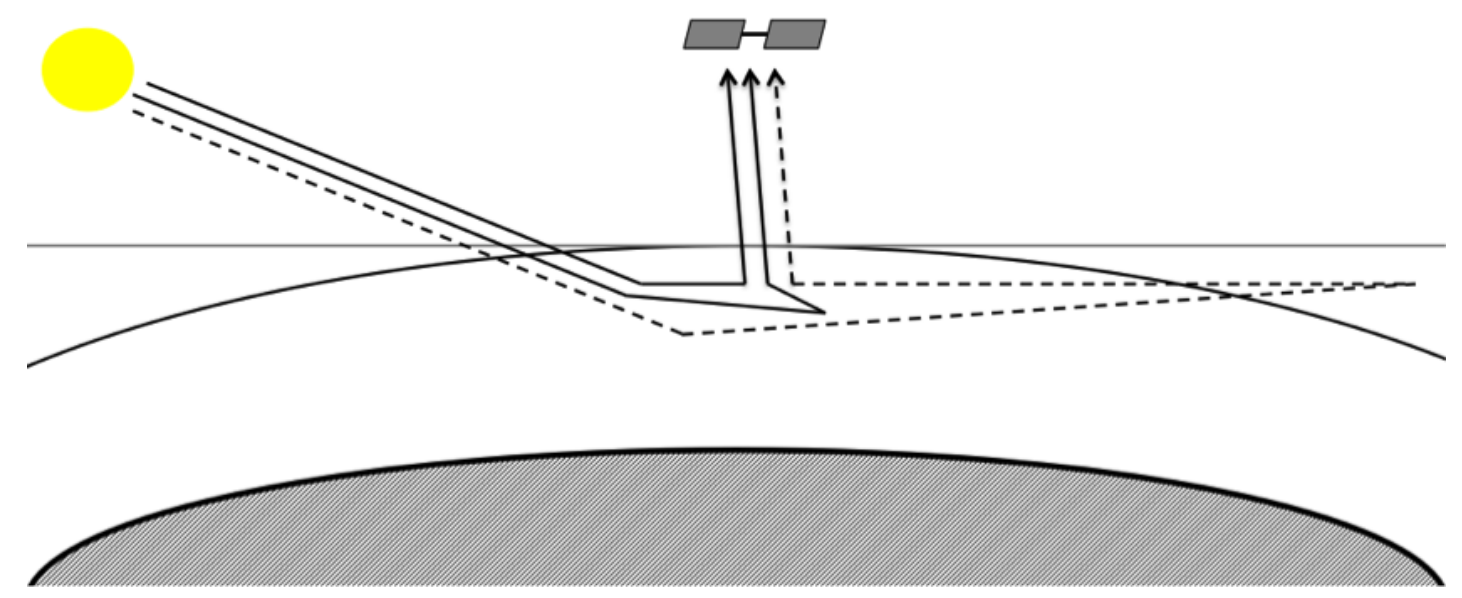

Figure 2.7: Schematic diagram of multiple scattering contributions to TOA reflectance in different RTMs. For DAK, LIDORT, and SCIATRAN all three photon paths are possible, whereas for McArtim only multiple scattering contributions from the spherical field (area bound by the solid grey and curved black TOA line) are possible.

altitude-dependent AMF show a non-linear dependency to surface albedo. Therefore we increased the number of reference points for albedo values between 0 and 0.2 . The vertical discretization of box-AMFs is also relevant, especially in the lower troposphere where strong gradients in the concentration of $\mathrm{NO}_{2}$ occur. Therefore, the vertical dimension of the LUT should have a fine resolution in the lower first few kilometres. For the other parameters, AMFs dependencies are relatively weak compared to the albedo and vertical sensitivity, therefore it is not necessary to increase the number of reference points (relative to the current LUT in DOMINO v2).

This section shows that the QA4ECV $\mathrm{NO}_{2}$ retrieval incorporates relevant improvements and it also provides a better quantification and understanding of the uncertainties in all the retrieval steps. The spectral fitting now accounts for liquid water absorption and includes an intensity offset correction, which improved the quality of the fits over background ocean scenes by 30\% (Zara et al., 2018). A major step in the data assimilation and tropospheric air mass factor calculation has been the use of the CTM TM5 at a spatial resolution of $1^{\circ} \times 1^{\circ}$. The stratospheric estimation has also benefited from the sphericity correction applied in the AMF calculation. These improvements lead to around $15 \%$ fewer negative $\mathrm{NO}_{2}$ columns. Most of the developments from the QA4ECV retrieval have been implemented in the TROPOMI $\mathrm{NO}_{2}$ retrieval algorithm, although the spectral fitting is with the intensity fit as in van Geffen et al. (2015) and the intensity offset correction has not been implemented yet. 


\section{3 $\quad \mathrm{NO}_{2} \mathrm{QA} 4 \mathrm{ECV}$ climate data record}

Traceable information on the quality of the QA4ECV NO 2 climate data record, on all the retrieval sub-processes, on the analysis methods, and on the validation steps is essential for the explotation of data by users with varying knowledge level. In order to give full traceability throughout the whole processing chain of the retrieval algorithm, traceability chains were created to describe the specifics of each step of the retrieval. In this interactive diagram, links and references to ancillary datasets used in the retrieval process are given as well. These diagrams are presented to users as a complement to the Algorithm Theoretical Baseline Document (ATBD), which are usually dense and difficult to read for users that are not familiar with the data production chain.

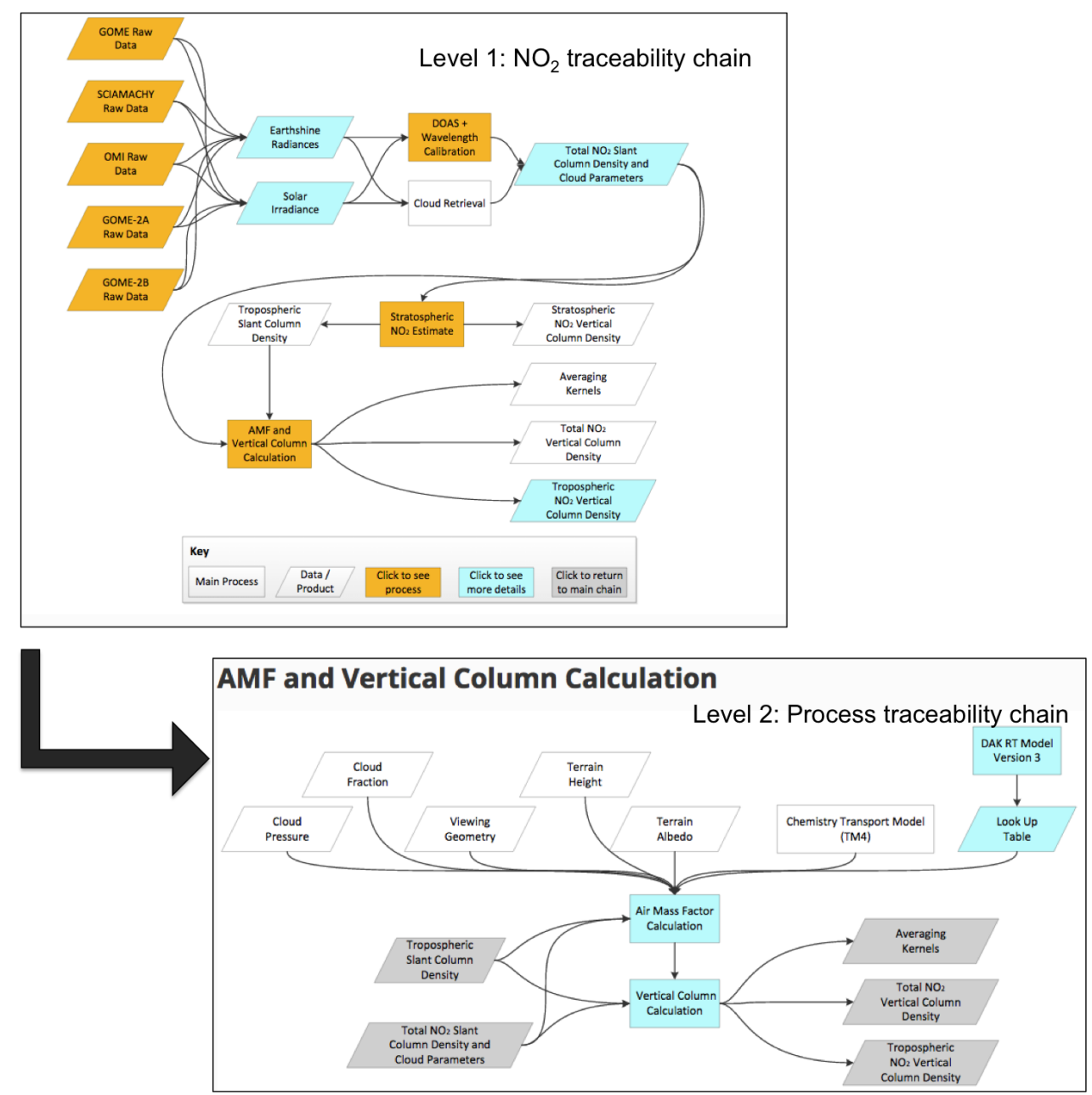

Figure 2.8: Traceability Chain for the QA4ECV $\mathrm{NO}_{2}$ retrieval algorithm. The rectangles are the main processes of the retrieval, and these are clickable to get more details of the sub-processes. The light blue blocks provide more information on the data. The interactive traceability chain is available at: http://www.qa4ecv.eu/ecv/no2-pre.

Figure 2.8 shows the $\mathrm{NO}_{2}$ traceability chain. The main entry is an overview of the 
Table 2.3: Ancillary data used in the AMF calculation of the QA4ECV $\mathrm{NO}_{2}$ retrieval algorithm.

\begin{tabular}{lllll}
\hline & $\begin{array}{l}\text { Terrain } \\
\text { height }\end{array}$ & $\begin{array}{l}\text { Surface } \\
\text { reflectivity }\end{array}$ & $\begin{array}{l}\text { Cloud } \\
\text { parameters }\end{array}$ & $\begin{array}{l}\mathrm{NO}_{2} \text { a priori } \\
\text { profile }\end{array}$ \\
\hline $\begin{array}{l}\text { GOME } \\
\text { SCIAMACHY } \\
\text { GOME-2A }\end{array}$ & $\begin{array}{l}\text { Global 3km } \\
\text { DEM* }\end{array}$ & $\begin{array}{l}\text { Min LER } \\
\text { Tilstra et al. } \\
(2017)\end{array}$ & FRESCO+ & $\begin{array}{l}\text { Daily TM5 } \\
\left(1^{\circ} \times 1^{\circ}\right)\end{array}$ \\
\hline OMI & $\begin{array}{l}\text { Global 3km } \\
\text { DEM* }^{*}\end{array}$ & $\begin{array}{l}\text { Min LER } \\
\text { Kleipool et al. } \\
(2008)(\mathrm{v} 002)\end{array}$ & $\mathrm{O}_{2}-\mathrm{O}_{2}$ & $\begin{array}{l}\text { Daily TM5 } \\
\left(1^{\circ} \times 1^{\circ}\right)\end{array}$ \\
\hline
\end{tabular}

${ }^{*}$ Digital Elevation Model data.

retrieval algorithm that give access to all sub-steps. All the orange boxes in the first level of the traceability chain represent different sub-processes in the retrieval algorithm, and by clicking on them the user can access the second level of the traceability chain. An example of one of the sub-process is shown in Fig. 2.8 for the "AMF and vertical column calculation", which gives detailed information about all the steps (e.g. radiative transfer modelling, AMF calculation) and the external information (e.g. surface albedo, cloud parameters) that is used. The forward model input parameters that have been used for the QA4ECV retrieval are state-of-the-art datasets that are available for the full processing period (1995 - 2017), and are summarized in Table 2.3.

The QA4ECV NO 2 1995-2017 climate data record ${ }^{2}$ is publicly available and can be found at www.qa4ecv.eu and www.temis.nl. The processing of more than 20 years of data was led by KNMI and supported by IASB-BIRA, IUP Bremen, Max Planck Institute for Chemistry (MPI-C), and Wageningen University (WUR), and each of them played a crucial role in different parts of the processing. Slant column densities were produced by IASB-BIRA and IUP Bremen, stratospheric fields from STREAM were computed at KNMI with MPI-C software, WUR created the AMF LUT and the central processing was performed at KNMI, where all the inputs where brought together.

The QA4ECV data record starts in 1995 with GOME and although the project only processed data until 2017, QA4ECV retrieval algorithm is being applied to OMI from 2017 onwards (this data is available via temis.nl together with the DOMINO v2 data). Figure 2.9 shows GOME tropospheric $\mathrm{NO}_{2}$ in September 1995 and OMI tropospheric $\mathrm{NO}_{2}$ in September 2018. Thanks to QA4ECV, any user can directly compare these 23 year apart $\mathrm{NO}_{2}$ fields knowing that they are fully consistent with respect to the algorithm that has been applied to obtain the results. This is the first time that such dataset is available to the public, and it is a very powerful tool not only to scientists outside the field of

\footnotetext{
${ }^{2} \mathrm{~A}$ climate data record is a time series of a variable that can be monitored, in this case with Earth Observation systems, and that is associated with climate change (NRC, 2004)
} 


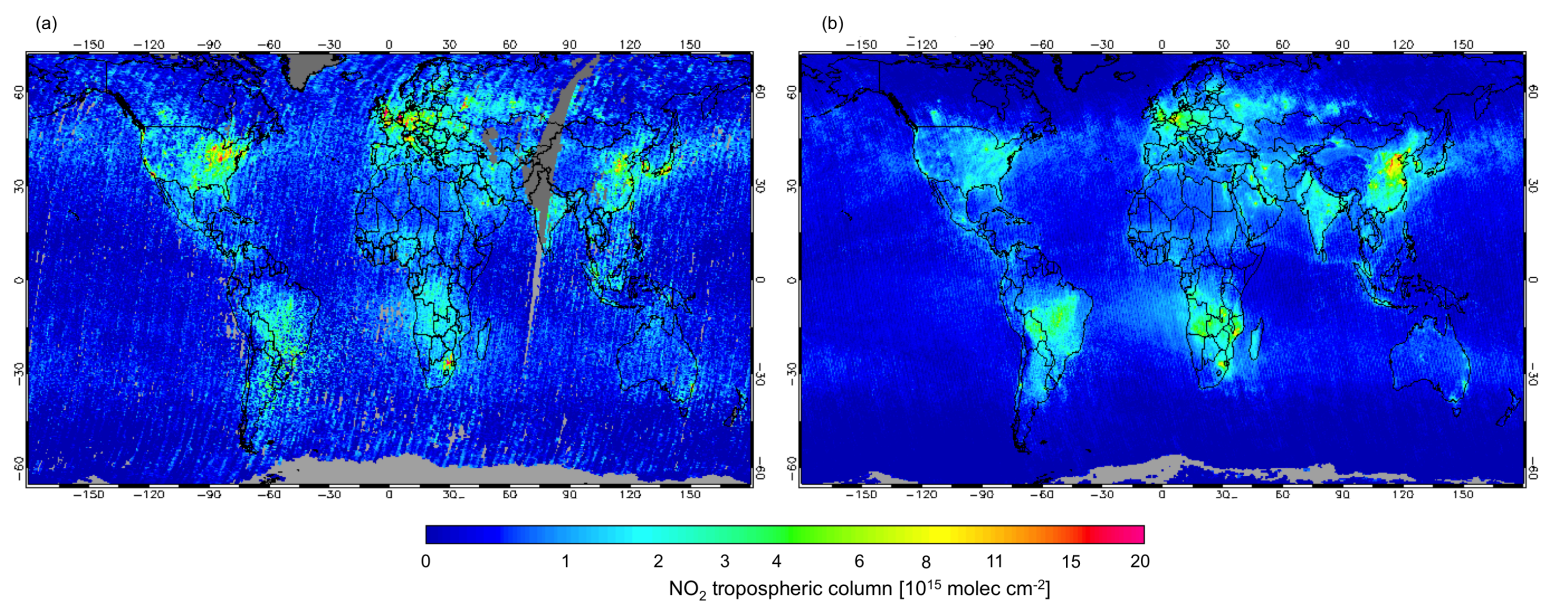

Figure 2.9: QA4ECV $\mathrm{NO}_{2}$ tropospheric vertical column from (a) GOME for September 1995 and from (b) OMI for September 2018. Pictures are taken from www.qa4ecv.eu for GOME and www.temis.nl/airpollution/no2col for OMI.

satellite retrievals, but also to companies and policy makers that want to use air quality data for different purposes. Several scientific studies have already used QA4ECV data, like in the multi-constituent data assimilation study by Miyazaki et al. (2018).

\subsection{QA4ECV $\mathrm{NO}_{2}$ product validation}

In this section we compare QA4ECV $\mathrm{NO}_{2}$ tropospheric vertical columns to the DOMINO v2 retrieval and to ground-based MAX-DOAS measurements. The comparison with DOMINO v2 allows us to evaluate the effect of the retrieval improvements applied in the QA4ECV retrieval. As the results shown in Sect. 2.2 point to better $\mathrm{NO}_{2}$ columns resulting from the QA4ECV retrieval, we expect that the agreement with independent ground-based measurements will improve with respect to DOMINO v2.

\subsubsection{Comparison to DOMINO}

For the comparison with DOMINO v2, we select OMI measurements from $2005^{2}$. Figure 2.10 shows yearly mean tropospheric $\mathrm{NO}_{2}$ vertical column density from OMI retrieved with the QA4ECV algorithm and DOMINO v2 algorithm. Both QA4ECV and DOMINO v2 $\mathrm{OMI} \mathrm{NO}$ data has been gridded to a $0.04^{\circ}$ x $0.08^{\circ}$ latitude - longitude grid. To assure that mostly clear-sky measurements are taken into account, only measurements with a cloud radiance fraction lower than $50 \%$ for both retrievals have been used to calculate

\footnotetext{
${ }^{2} 2005$ is at the beginning of the mission and the instrument was not yet affected by the row anomaly that influences the quality of the radiances for certain rows from 2007.
} 
the mean $\mathrm{NO}_{2}$ tropospheric columns. To avoid scenes contaminated by ice or snow, only pixels with a surface albedo lower than 0.3 are considered.
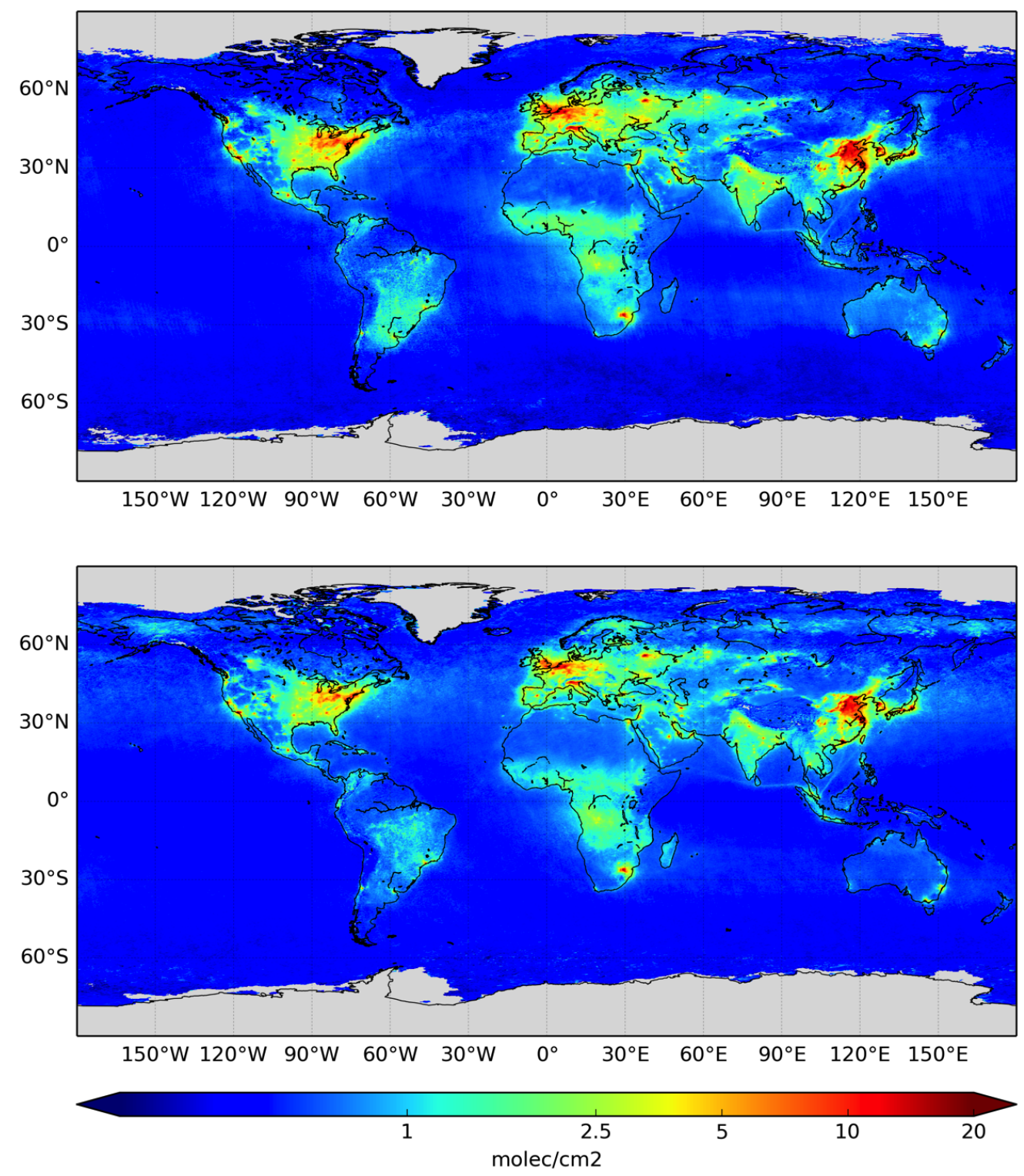

Figure 2.10: Yearly mean $\mathrm{NO}_{2}$ tropospheric vertical column in 2005 from OMI retrieved with DOMINO v2 (upper panel) and QA4ECV (lower panel) averaged in a $0.04^{\circ} \times 0.08^{\circ}$ latitude - longitude grid.

Figure 2.11 shows the absolute differences between QA4ECV and DOMINO v2 $\mathrm{NO}_{2}$ tropospheric VCDs from OMI for 2005. Globally for 2005, QA4ECV NO 2 values are $9.5 \%$ higher than DOMINO v2, and the correlation coefficient between the two datasets is $\mathrm{R}^{2}=$ 0.93 (number of pixels around $10^{7}$ ). The higher values of QA4ECV correspond mainly to 
background areas (red zones in Fig. 2.11), where the tropospheric slant column densities are higher due to the (improved) lower stratospheric columns.

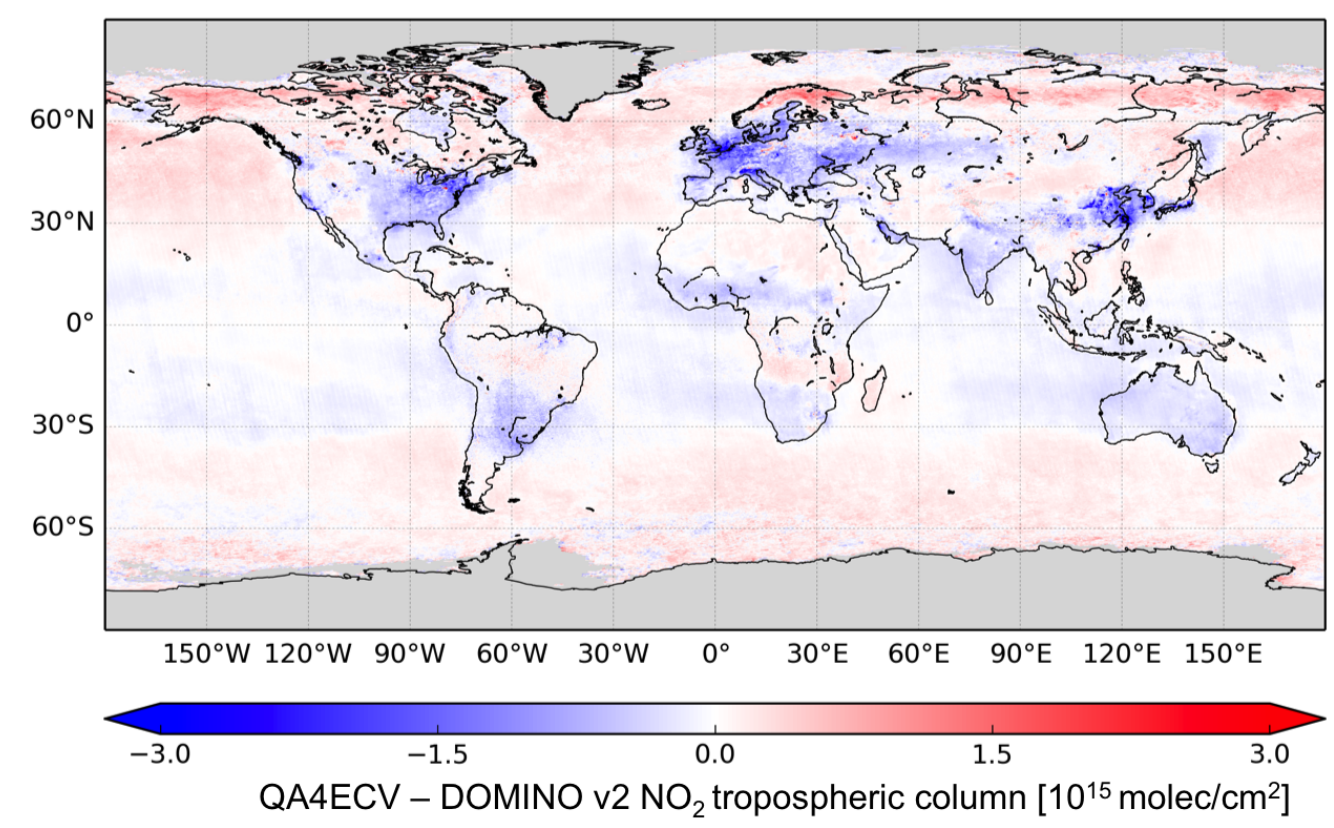

Figure 2.11: Absolute differences of $\mathrm{NO}_{2}$ tropospheric vertical column retrieved with QA4ECV and DOMINO from OMI for 2005, averaged in a $0.04^{\circ} \times 0.08^{\circ}$ latitude - longitude grid.

Over the polluted urban areas of Europe, United States and Asia, QA4ECV $\mathrm{NO}_{2}$ values are lower than DOMINO v2 (blue spots in Fig. 2.11), mainly due to higher AMF values in QA4ECV. Figure 2.12 shows a correlation plot for each of these regions. The correlation coefficient between the datasets for these particular regions is higher than $\mathrm{R}^{2}$ $=0.95$ (number of pixels for each region is around $4 \cdot 10^{5}$ ). An example of QA4ECV and DOMINO v2 $\mathrm{NO}_{2}$ tropospheric columns over Europe and their differences is shown in Fig. 2.13. QA4ECV $\mathrm{NO}_{2}$ VCDs are $20 \%$ lower than DOMINO v2 over Europe. The difference map shows that over highly polluted areas QA4ECV is significantly lower than DOMINO, like over the Po-Valley and the English channel. Also the outskirts of cities like Madrid or Barcelona are visible in the differences plot, and also smaller cities in North-West Spain.

One of the main improvements of QA4ECV retrieval algorithm with respect to DOMINO v2 is the use of a higher resolution a priori profile (TM4 at $3^{\circ} \times 2^{\circ}$ and TM5 at $1^{\circ} \times 1^{\circ}$ ). This is clearly visible in Fig. 2.14 over the city of Paris, where the contrast between the high polluted Paris and the background is much stronger than in DOMINO v2. This results also in better resolved shipping lanes in QA4ECV (see the Indian ocean 

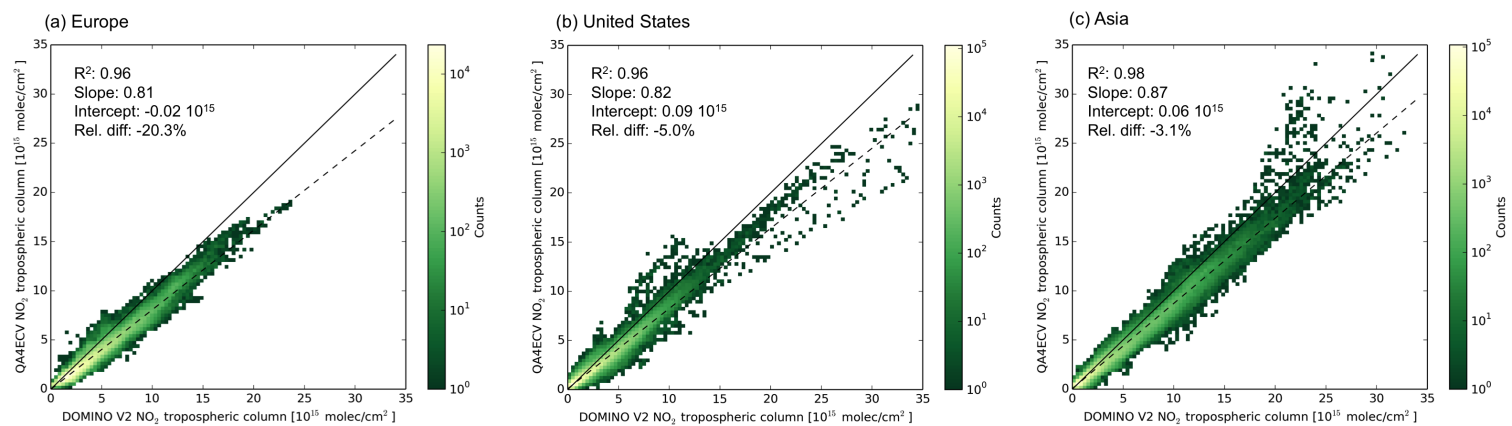

Figure 2.12: Scatter plot of QA4ECV and DOMINO v2 $\mathrm{NO}_{2}$ tropospheric vertical column in 2005 from OMI over (a) Europe $\left(35^{\circ}-60^{\circ} \mathrm{N}, 10^{\circ} \mathrm{W}-30^{\circ} \mathrm{E}\right)$, (b) United States $\left(25^{\circ}-55^{\circ} \mathrm{N}\right.$, $\left.70^{\circ}-130^{\circ} \mathrm{W}\right)$ and (c) Asia $\left(20^{\circ}-50^{\circ} \mathrm{N}, 80^{\circ}-130^{\circ} \mathrm{E}\right)$.

(a)

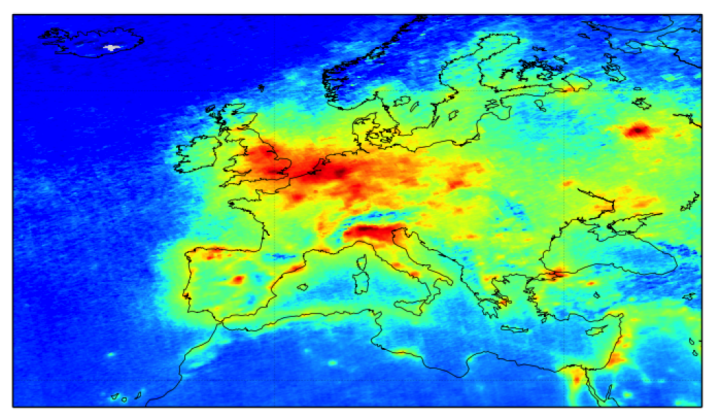

(b)

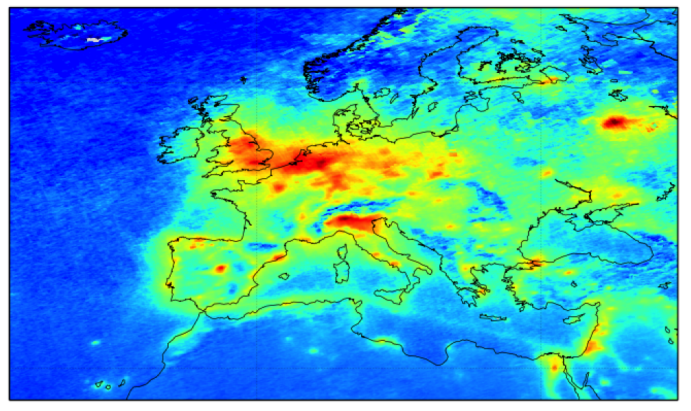

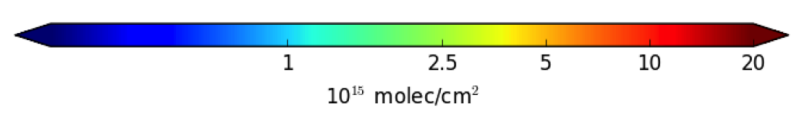

(c)

QA4ECV - DOMINO v2

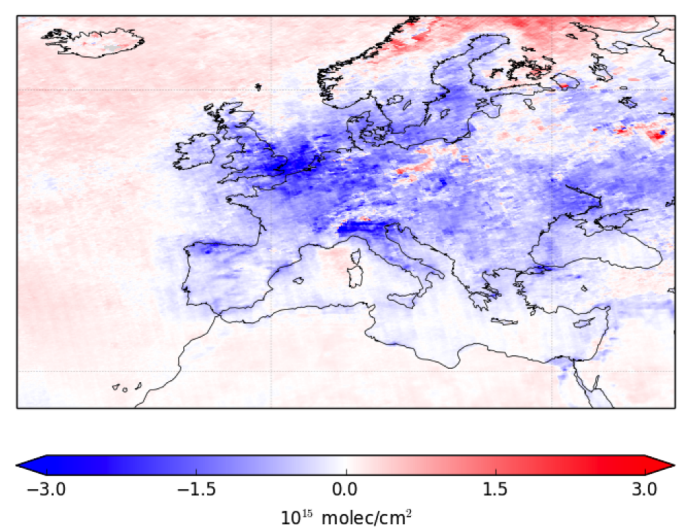

Figure 2.13: Yearly mean $\mathrm{NO}_{2}$ tropospheric vertical column in 2005 from OMI over Europe retrieved with (a) DOMINO v2 and (b) QA4ECV averaged in a $0.04^{\circ} \times 0.08^{\circ}$ latitude longitude grid. (c) Absolute differences between QA4ECV and DOMINO v2 $\mathrm{NO}_{2}$ tropospheric vertical column in 2005. 

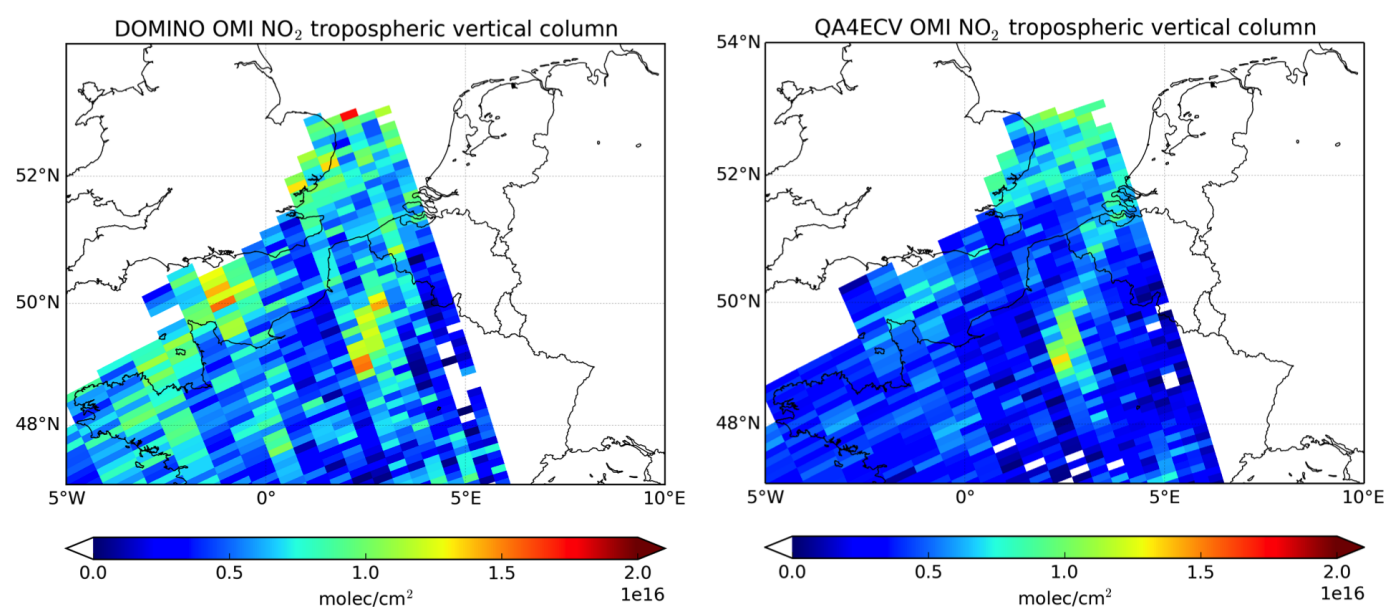

Figure 2.14: $\mathrm{NO}_{2}$ tropospheric vertical column from DOMINO v2 (left) and QA4ECV (right) retrieval algorithms, on the $22^{\text {nd }}$ of November 2017.

in 2.10 as an example). The improved spectral fitting results in a less noisy slant column densities, as shown in 2.15(c,d), with absolute SCD levels that agree within 10\%. Figure 2.15(a,b) shows that QA4ECV AMFs are higher than DOMINO v2 AMFs. Differences in cloud parameters, albedo and TM5 $\mathrm{NO}_{2}$ profile shape may have contributed to the AMF differences between DOMINO v2 and QA4ECV, which is currently being investigated.

The comparison of QA4ECV $\mathrm{NO}_{2}$ with DOMINO v2 shows lower QA4ECV values over polluted areas. Local pollution is better represented in QA4ECV because of the better contrast between background and highly polluted areas. This is a direct consequence of the improved slant column densities and the higher resolution of the CTM in the data assimilation system and in the AMF calculation. Together with the QA4ECV NO 2 tropospheric column the averaging kernels are also provided. Averaging kernels represent the relation between the vertical column and the slant column density without the influence of the a priori profile (Eskes and Boersma, 2003), and thus they should be used to recalculate the air mass factor using an a priori profile that is more suitable for a particular model to satellite comparison.

\subsubsection{QA4ECV Validation}

As a preliminary validation effort and to asses whether the improvements in QA4ECV point in the right direction, here we compare QA4ECV OMI tropospheric $\mathrm{NO}_{2}$ columns with independent MAX-DOAS column measurements in the city of Tai'an (China) and the De Bilt (The Netherlands). The MAX-DOAS measurements in Tai'an were done by Irie et al. (2008) in May-June 2006, and the measurements over De Bilt in July 2014. 
(a)

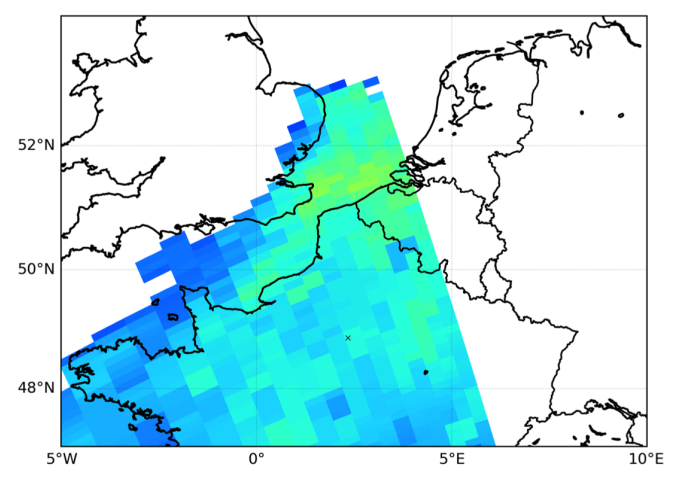

(b)

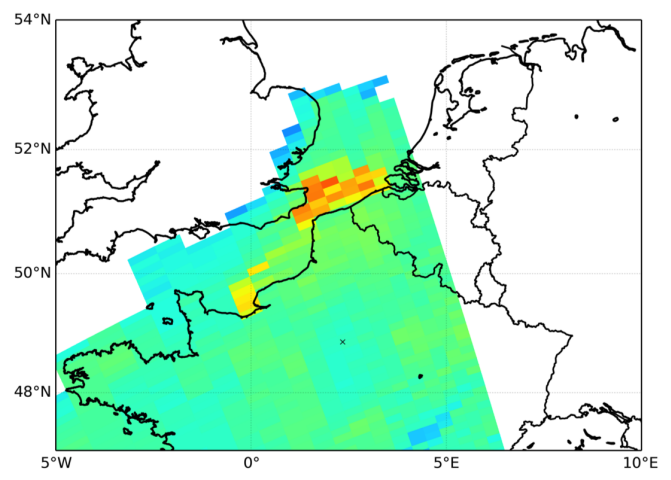

(c)

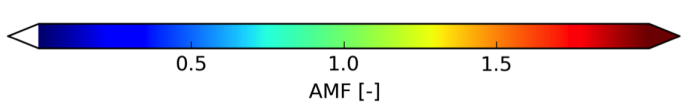

(d)
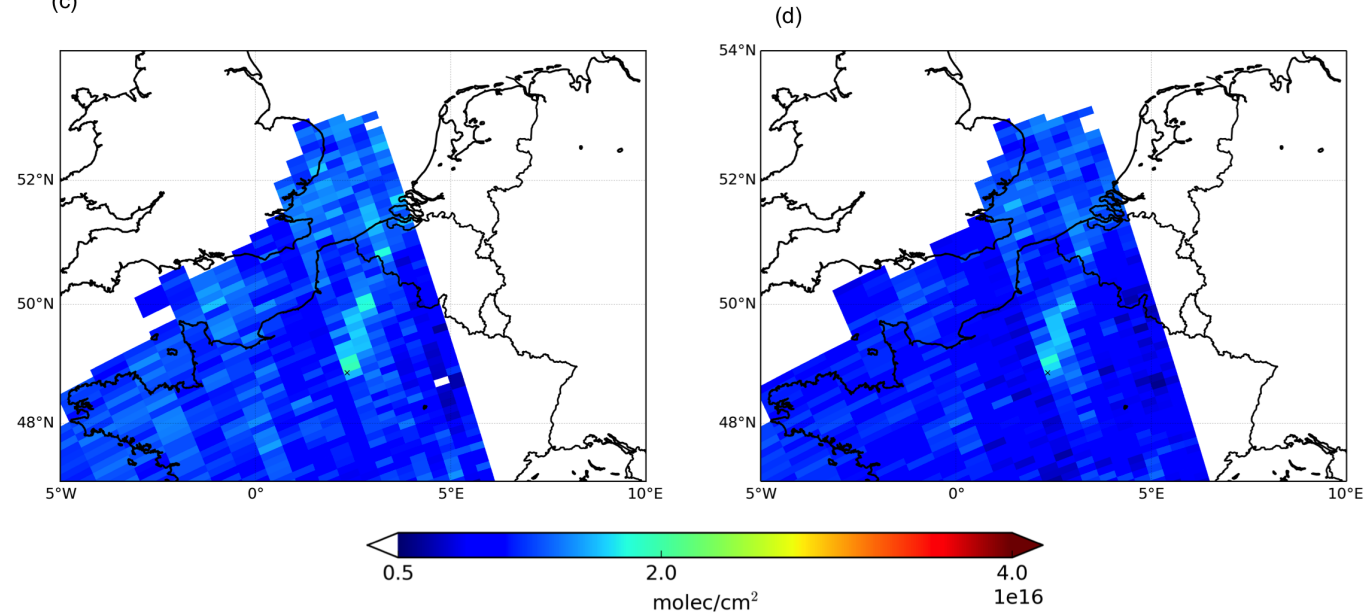

Figure 2.15: (a, b) Air mass factor and slant column densities (b, c) from DOMINO v2 (left) and QA4ECV (right) retrieval algorithms on the $22^{\text {nd }}$ of November 2017.

Uncertainties in the MAX-DOAS $\mathrm{NO}_{2}$ columns are driven by noise, air mass factor and temperature uncertainties amounting to approximately $15 \%$ uncertainty.

We compare OMI pixels with a collocation distance of $20 \mathrm{~km}$ and 30 minutes of the MAX-DOAS measurements. The collocation criterion limits spatial representativeness mismatches between the two measurements, and is consistent with the spatial dimensions of the MAX-DOAS $( \pm 10 \mathrm{~km})$ and OMI $(20-30 \mathrm{~km})$ footprints. We also require that the satellite retrieval is performed under mostly clear-sky conditions, with a cloud radiance fraction lower than $50 \%$. We also discard pixels with effective cloud pressure higher than $875 \mathrm{hPa}$, as they might be contaminated by aerosols (mostly relevant for validation in cities with high concentration of particulate matter, like Tai'an).

Figure 2.16(a) shows a scatter plot of QA4ECV and MAX-DOAS $\mathrm{NO}_{2}$ tropospheric columns. We find a mean difference of $-0.57 \cdot 10^{15}$ molec $\cdot \mathrm{cm}^{-2}(-7 \%)$. The regression 
analysis gives a slope of 0.85 and an intercept of $-0.4 \cdot 10^{15} \mathrm{molec} \cdot \mathrm{cm}^{-2}$, with a correlation coefficient of 0.3 . Figure 2.16(b) shows a scatter plot of DOMINO v2 and MAX-DOAS $\mathrm{NO}_{2}$ tropospheric columns. We find a mean difference of $1.02 \cdot 10^{15} \mathrm{molec} \cdot \mathrm{cm}^{-2}(18 \%)$. The regression analysis gives a slope of 0.8 and an intercept of $2.4 \cdot 10^{15} \mathrm{molec} \cdot \mathrm{cm}^{-2}$ with a correlation coefficient of 0.14. DOMINO v2 has higher number of collocated pixels with MAX-DOAS because DOMINO v2 uses the previous version of the OMI $\mathrm{O}_{2}-\mathrm{O}_{2}$ cloud product (Acarreta et al., 2004), which has too low cloud pressures (Veefkind et al., 2016).

(a)

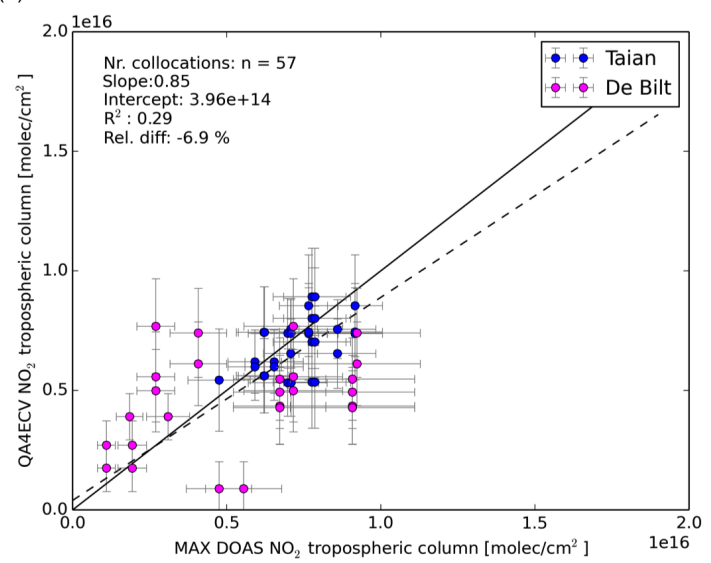

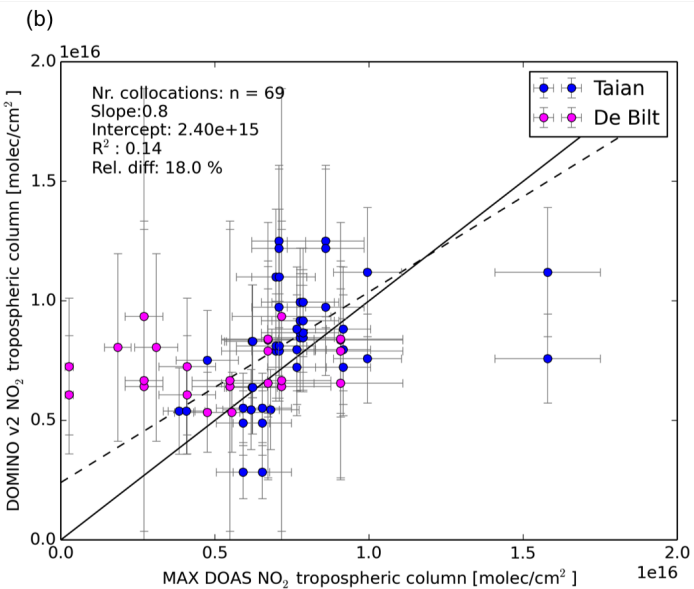

Figure 2.16: (a) Scatter-plot of QA4ECV OMI and (b) DOMINO v2 vs. MAX-DOAS tropospheric $\mathrm{NO}_{2}$ columns for Tai'an (blue) (China) in May-June 2006 and De Bilt (pink) (The Netherlands) in July 2014. The solid line shows the 1-1 line and the dashed line is the result of a reduced major axis regression to the data. Only pixels with a cloud radiance fraction lower than 0.5 , an effective cloud pressure lower than $875 \mathrm{hPa}$, within $20 \mathrm{~km}$ and 30 minutes of a MAX-DOAS measurement have been selected.

In line with the QA4ECV and DOMINO v2 comparison in the previous section, we see that positive bias of the DOMINO v2 $\mathrm{NO}_{2}$ columns with respect to MAX-DOAS $(+18 \%)$ is reduced and changes sign for QA4ECV $(-7 \%) \mathrm{NO}_{2}$ columns. The discrepancy between OMI and MAX-DOAS measurements in moderately to highly polluted areas like Tai'an and De Bilt is mainly due to the spatial inhomogeneity in the $\mathrm{NO}_{2}$ columns measured around the ground based stations. The better agreement with OMI QA4ECV product is mainly due to lower QA4ECV columns. This first validation is based on a limited time range and only for two sites, so the sample size is small. A more comprehensive validation work based on several MAX-DOAS sites and multiple years is ongoing by the QA4ECV validation team (Compernolle et al., 2018). 


\subsection{Conclusions}

In the QA4ECV project, in which several leading satellite retrieval groups participated, we have developed an improved community best practice retrieval algorithm that has been applied to four different UV/Vis sensors to generate a harmonized 1995-2017 $\mathrm{NO}_{2}$ data record. The dataset also contains traceable information on each of the retrieval steps and a full characterization of the uncertainties, which is of central importance when using satellite measurements for data assimilation and inverse modelling studies.

The improvements achieved in QA4ECV for the $\mathrm{NO}_{2}$ (and $\mathrm{HCHO}$ ) retrieval algorithms are based on a thorough comparison of state-of-the-art retrievals. During the algorithm development phase, to establish the best approach for each retrieval step, dedicated intercomparison experiments were performed. The comparison results were extensively discussed and have been documented in detail in deliverables and peer-reviewed publications that are publicly available. Also the fitness for purpose of the QA4ECV dataset has been evaluated in peer-reviewed publications (Miyazaki et al., 2018) as well as in several studies from $\mathrm{PhD}$ and MSc students (Boersma et al., 2016a). Most of the developments in the QA4ECV retrieval algorithm have been implemented in the retrieval algorithm for the TROPOMI instrument. Within QA4ECV we were very close to the TROPOMI team and the verification group, which resulted in intensive scientific collaboration.

In the QA4ECV retrieval algorithm, the spectral fitting method now includes liquid water absorption and an intensity offset correction. Due to the more accurate spectral fitting, the quality of the fits has substantially improved especially over background scenes, and the uncertainties of the slant column densities have decreased (Zara et al., 2018). For stratospheric estimation, the data assimilation approach was selected to give consistency to the retrieval algorithm. A relevant improvement in this step has been the higher resolution $\mathrm{CTM}$ (from $3^{\circ} \times 2^{\circ}$ to $1^{\circ} \times 1^{\circ}$ ) and the sphericity correction in the radiative transfer model. This new implementation results in less unphysical negative $\mathrm{NO}_{2}$ tropospheric columns, a problem that was present in the DOMINO retrieval. The sphericity correction was an outcome of the comparison made within QA4ECV of the radiative transfer models for the AMF calculation. Specific differences between the pseudo spherical RTMs with the McArtim full-spherical model pointed to the importance of accounting for Earth's sphericity, particularly for extreme geometries and for stratospheric AMFs. The AMF calculation has greatly benefited from the improved resolution of the CTM used for the a priori profiles.

All the improvements resulted in smaller (5-20\%) QA4ECV OMI $\mathrm{NO}_{2}$ vertical column densities over polluted areas as compared to the ones retrieved with DOMINO v2. Highly polluted cities like Paris can be resolved better in QA4ECV due to the higher resolution a priori profiles and the lower and less noisy slant column densities in the surroundings of the 
cities. In the remote background areas, QA4ECV has a better estimation of stratospheric fields due to the sphericity correction, which makes QA4ECV tropospheric columns higher than DOMINO v2. QA4ECV NO 2 columns over Tai'an and De Bilt agree within 7\% with ground based MAX-DOAS measurements. The comparison shows that the lower QA4ECV tropospheric $\mathrm{NO}_{2}$ columns agree better with the MAX-DOAS measurements than the high biased (18\%) DOMINO v2 columns.

QA4ECV has generated a harmonized 1995-2017 data record of the ECV precursor $\mathrm{NO}_{2}$ (and also $\mathrm{HCHO}$ and $\mathrm{CO}$ ). The QA4ECV effort was complementary to the European Space Agency (ESA) Climate Change Initiative (CCI), who provided long-term satellite based data record of climate ECVs, not only for atmospheric ECVs but also for oceanic and terrestrial ECVs. This shows the strong potential and relevance of remote sensing measurements to help not only scientists to improve their understanding on the climate system, but also as a tool to bring science and policy together to face current and future environmental challenges. In order to serve this purpose, the European Commission, ESA and other institutions need to work together and empower the scientific community. 



\section{Structural uncertainty in air mass factor calculation for $\mathrm{NO}_{2}$ and HCHO satellite retrievals}

This chapter is published as:

Lorente, A., Boersma, K. F., Yu, H., Dörner, S., Hilboll, A., Richter, A., Liu, M., Lamsal, L. N., Barkley, M., De Smedt, I., Van Roozendael, M., Wang, Y., Wagner, T., Beirle, S., Lin, J.-T., Krotkov, N., Stammes, P., Wang, P., Eskes, H. J., and Krol, M.: Structural uncertainty in air mass factor calculation for $\mathrm{NO}_{2}$ and $\mathrm{HCHO}$ satellite retrievals, Atmos. Meas. Tech., 10, 759-782, doi: 10.5194/amt-10-759-2017, 2017 


\section{Abstract}

Air mass factor (AMF) calculation is the largest source of uncertainty in $\mathrm{NO}_{2}$ and $\mathrm{HCHO}$ satellite retrievals in situations with enhanced trace gas concentrations in the lower troposphere. Structural uncertainty arises when different retrieval methodologies are applied in the scientific community to the same satellite observations. Here, we address the issue of AMF structural uncertainty via a detailed comparison of AMF calculation methods that are structurally different between seven retrieval groups for measurements from the Ozone Monitoring Instrument (OMI). We estimate the escalation of structural uncertainty in every sub-step of the AMF calculation process. This goes beyond the algorithm uncertainty estimates provided in state-of-the-art retrievals, which address the theoretical propagation of uncertainties for one particular retrieval algorithm only. We find that top-of-atmosphere reflectances simulated by four radiative transfer models (RTMs) (DAK, McArtim, SCIATRAN and VLIDORT) agree within 1.5\%. We find that different retrieval groups agree well in the calculations of altitude resolved AMFs from different RTMs (to within 3\%), and in the tropospheric AMFs (to within 6\%) as long as identical ancillary data (surface albedo, terrain height, cloud parameters and trace gas profile) and cloud and aerosol correction procedures are being used. Structural uncertainty increases sharply when retrieval groups use their preference for ancillary data, cloud and aerosol correction. On average, we estimate the AMF structural uncertainty to be $42 \%$ over polluted regions and $31 \%$ over unpolluted regions, mostly driven by substantial differences in the a priori trace gas profiles, surface albedo and cloud parameters. Sensitivity studies for one particular algorithm indicate that different cloud correction approaches result in substantial AMF differences in polluted situations ( 5 to $40 \%$ depending on cloud fraction and cloud pressure, and $11 \%$ on average) even for low cloud fractions (lower than 0.2) and the choice of aerosol correction introduces an average uncertainty of $50 \%$ for situations with high pollution and high aerosol loading. Our work shows that structural uncertainty in AMF calculations is significant and that is mainly caused by the assumptions and choices made to represent the state of the atmosphere. In order to decide which approach and which ancillary data are the best for AMF calculations, we call for well-designed validation exercises focusing on polluted situations when AMF structural uncertainty has the highest impact on $\mathrm{NO}_{2}$ and $\mathrm{HCHO}$ retrievals. 


\subsection{Introduction}

Satellite observations in the UV and visible spectral range are widely used to monitor trace gases such as nitrogen dioxide $\left(\mathrm{NO}_{2}\right)$ and formaldehyde $(\mathrm{HCHO})$. These gases are relevant for air quality and climate change, because they are involved in the formation of tropospheric ozone and aerosols, which have an important influence on atmospheric radiative forcing (Myhre et al., 2013). Ozone and aerosols are defined as "essential climate variables" (ECVs) by the Global Climate Observing System (GCOS). These ECVs and their precursors $\left(\mathrm{NO}_{2}\right.$ and $\mathrm{HCHO}$ among others) are included in the ECV framework because they contribute to characterization of Earth's climate and they can be monitored from existing observation systems (Bojinski et al., 2014). Currently a wide range of ECV products are available, but they rarely have reliable and fully traceable quality information. To address this need, the Quality Assurance for Essential Climate Variables project (QA4ECV, www.qa4ecv.eu) aims to harmonize, improve and assure the quality of retrieval methods for the ECV precursors $\mathrm{NO}_{2}$ and $\mathrm{HCHO}$. Here, we focus on retrievals of tropospheric $\mathrm{NO}_{2}$ and $\mathrm{HCHO}$ vertical column densities (VCDs) from spaceborne UV/Vis spectrometers. Retrievals from these instruments have been used for a wide range of applications. These notably include estimating anthropogenic emissions of $\mathrm{NO}_{x}$ and $\mathrm{HCHO}$ (e.g. Boersma et al. (2015), Marbach et al. (2009)), natural isoprene emissions (e.g. Marais et al. (2014), Barkley et al. (2013)) and $\mathrm{NO}_{x}$ production from lightning (e.g. Lin (2012), Beirle et al. (2010)), data assimilation (e.g. Miyazaki et al. (2012)), and trend detection (e.g. Krotkov et al. (2016), Richter et al. (2005), De Smedt et al. (2010)).

Although trace gas satellite retrievals have improved over the last decades (e.g. Li et al. (2015), Richter et al. (2011), De Smedt et al. (2012), Bucsela et al. (2013)), there is still a need for a more complete understanding of the uncertainties involved in each retrieval step. The retrieval of $\mathrm{NO}_{2}$ and $\mathrm{HCHO}$ columns consists of three successive steps. First a spectral fitting is performed to obtain the trace gas concentration integrated along the average atmospheric light path (slant column density, SCD) from backscattered radiance spectra. For $\mathrm{NO}_{2}$, the stratospheric contribution to the SCD is removed to obtain the tropospheric SCD. Finally, the SCD is converted into the vertical column density (VCD) using an air mass factor (AMF). Previous studies indicated that the AMF calculation is the largest source of uncertainty (contributing up to half of the typical VCD uncertainties of 40-60\%) in the $\mathrm{NO}_{2}$ and $\mathrm{HCHO}$ retrievals in scenarios with a substantial tropospheric contribution to the total column (e.g. Boersma et al. (2004), De Smedt et al. (2008), Barkley et al. (2012)). These studies arrived at such theoretical uncertainty estimates based on error propagation for one specific retrieval algorithm.

Theoretical uncertainty (also known as parametric uncertainty) is the uncertainty arising within one particular retrieval method. Structural uncertainty is the uncertainty 
that arises when different retrieval methodologies are applied to the same data (Thorne et al., 2005). To represent the state of the atmosphere, several choices and assumptions are made in the retrieval algorithm, in particular within the AMF calculation. Even though these choices are physically robust and valid, when different retrieval algorithms based on different choices are applied to the same satellite observations, this usually leads to different results. The structural uncertainty is intrinsic to the retrieval algorithm formulation and it is considered to be a source of systematic uncertainty (Povey and Grainger, 2015). In principle, theoretical and structural uncertainties should be considered independently from each other. However, in the calculation of the theoretical uncertainty, the contribution of the ancillary data is often calculated comparing different databases (e.g. to estimate surface albedo uncertainty as in Boersma et al. (2004)) rather than using the uncertainty of the database itself. Consequently, some components are shared in the structural and theoretical uncertainty calculations. However, for a full structural uncertainty estimate, all sources of methodological differences need to be considered. In the framework of AMF calculations addressed here, this implies e.g. the selection of radiative transfer model, vertical discretization and interpolation schemes, the method for cloud and aerosol correction and the selection of (external or ancillary) data on the atmospheric state (surface reflectivity, cloud cover, terrain height, and a priori trace gas profile). The problem of structural uncertainty has been addressed in other fields of atmospheric sciences, e.g. in satellite retrievals for atmospheric variables (Fangohr and Kent, 2012) and in numerical models for climate studies (Tebaldi and Knutti, 2007).

There are few studies addressing structural uncertainty for trace gas retrievals. Van Noije et al. (2006) compared $\mathrm{NO}_{2}$ tropospheric columns retrieved from GOME data by 3 different groups. In that study, the discrepancies inherent to differences and assumptions in the retrieval methods were identified as a major source of systematic uncertainty. However, the causes of discrepancies between retrievals were not addressed but were targeted for a more detailed investigation. In this study we focus on AMF structural uncertainty, by comparing the AMF calculation approaches by seven different retrieval groups and providing a traceable analysis of all components of the AMF calculation. Ensemble techniques to estimate structural uncertainty have already been applied in different atmospheric disciplines (e.g. Steiner et al. (2013), Liu et al. (2015)). The groups that participated in this study are: Belgian Institute for Space Aeronomy (IASB-BIRA; abbreviated as BIRA), Institute of Environmental Physics, University of Bremen (IUP-UB), Wageningen University (WUR) and Royal Netherlands Meteorological Institute (KNMI) (calculations made by WUR following the KNMI approach, abbreviated as WUR), University of Leicester (UoL), Max Planck Institute for Chemistry (MPI-C), NASA Goddard Space Flight Center (NASA-GSFC; abbreviated as NASA) and Peking University.

We start with a comparison of top-of-atmosphere (TOA) reflectances simulated by ra- 
diative transfer models (RTMs), the main tool for any AMF calculation (Sect. 3.3.1). The RTMs DAK, McArtim, SCIATRAN and VLIDORT solve the radiative transfer equation differently, and have different degrees of sophistication to account for Earth's sphericity and multiple scattering. Next we compare altitude-dependent (or box-) AMFs for $\mathrm{NO}_{2}$ and HCHO computed with the four RTMs (Sect. 3.3.2). This is followed by a comparison of tropospheric AMFs (for $\mathrm{NO}_{2}$ ) calculated by four groups for measurements by the Ozone Monitoring Instrument (OMI) based on identical settings (same ancillary data and same approach for cloud and temperature correction) (Sect. 3.3.3). We interpret the resulting spread between the tropospheric AMFs as the AMF structural uncertainty associated with using different RTMs, vertical discretization and interpolation schemes. Then, we investigate how the choice of cloud correction affects the AMF structural uncertainty (Sect. 3.3.3). For the overall structural uncertainty estimate, we perform a round robin exercise, in which seven different groups calculate $\mathrm{NO}_{2}$ AMFs using their own preferred methods for cloud and aerosol correction and sources of ancillary data. Here we asses the effect of the different choices in the AMF structural uncertainty. Finally, we investigate how stratospheric AMFs are affected by the selection of RTM and their physical description of photon transport through a spherical atmosphere. The complete chain of uncertainties associated with each phase provides traceable quality assurance for the AMF calculation. Recommendations on best practices are given for this particular algorithm step and they will be applied in a community best practice retrieval algorithm for ECV precursors, under development in the framework of the QA4ECV project.

\subsection{Methods}

\subsubsection{AMF calculation process}

The concept of traceability chain (here in the form of a flow diagram) for the AMF calculation process and uncertainty assessment used in this study is illustrated in Fig. 3.1. Structural uncertainty estimated in each step is based on the standard deviation $(1 \sigma)$ of relative differences of the compared elements. Modelled reflectance $(R)$ at TOA is the starting point for air mass factor calculations using radiative transfer models. A RTM solves the radiative transfer equation, which describes the transport of radiation through the atmosphere to the observer (in our case the satellite) and the physical processes that affect the intensity of the radiation (absorption, scattering, refraction and reflection) (first box in the diagram in Fig. 3.1). Reflectance (unitless) is calculated from fundamental radiation quantities, and it is defined as the ratio of modelled Earth radiance $(I)$ (times $\pi)$ and the solar irradiance at TOA perpendicular to the solar beam $\left(E_{0}\right)$ multiplied by 


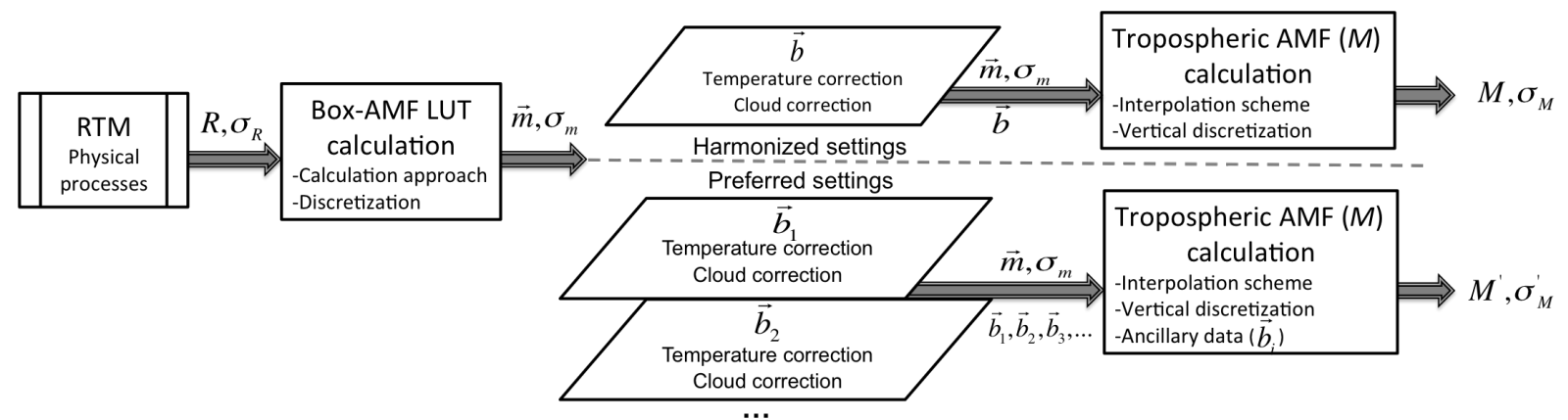

Figure 3.1: Flowchart of AMF calculation and comparison process followed in the study. In the third step forward model parameters $(\vec{b}$ : surface albedo, surface pressure, a priori profile, temperature, cloud fraction and cloud pressure) are selected for harmonized settings comparison (upper part) and preferred settings comparison (lower part). In each step the main differences between the compared elements are highlighted. The compared parameters and their structural uncertainty $(\sigma)$ in each step are: TOA reflectance $\left(R, \sigma_{R}\right)$, box-AMFs $\left(\vec{m}, \sigma_{m}\right)$, and tropospheric AMFs $\left(M, \sigma_{M}\right)$.

the cosine of the solar zenith angle $\left(\mu_{0}\right)$ :

$$
R(\lambda)=\frac{\pi I(\lambda)}{\mu_{0} E_{0}(\lambda)}
$$

Different models use different methods to solve the radiative transfer equation and to describe the sphericity of the Earth's atmosphere. Differences in modelled TOA reflectances between RTMs provide an estimate for the reflectance structural uncertainty $\left(\sigma_{R}\right)$. This uncertainty due to the choice of the RTM propagates to the next step in the AMF calculation.

Altitude dependent AMFs (box-AMFs, equivalent to scattering weights) characterize the vertical sensitivity of the measurement to a trace gas (e.g., Palmer et al. (2001)). They are directly related to how the measured radiance at TOA changes with a change of the optical depth of the atmosphere (related to the presence of a trace gas in a certain atmospheric layer), with the requirement that the absorber is optically thin (optical thickness $\tau_{\text {gas }} \ll 1$ ). In the context of the AMF calculation (second box in diagram of Fig. 3.1), box-AMFs for each layer can be calculated and stored in a look-up table (LUT) as a function of the forward model parameters (b) such as satellite viewing geometry, pressure level, surface pressure and surface reflectivity. There is also the possibility of online radiative transfer calculations for determining box-AMFs, i.e., bypassing the calculation of a LUT (e.g., Lin et al., (2014, 2015); Hewson et al. (2015)). Different RTMs use different vertical discretizations of the atmosphere, and calculate box-AMFs in different ways (see Sect. 3.2.2). A comparison of the box-AMF LUTs calculated with different RTMs provides a measure for the box-AMF structural uncertainty $\left(\sigma_{m}\right)$, which can be considered 
as the reproducibility of the box-AMFs from different RTMs when the same settings and input data are used.

The air mass factor $(M)$ represents the relative (dimensionless) length of the mean light path at a certain wavelength for photons interacting with a certain absorber in the atmosphere relative to the vertical path. The AMFs are used to convert the SCD obtained from the reflectance spectra to a VCD. To calculate the tropospheric VCD, a tropospheric $\mathrm{AMF}$ is used $\left(\mathrm{VCD}_{t r}=\mathrm{SCD}_{t r} / M_{t r}\right)$. But for species that have a stratospheric contribution to the total slant column, the stratospheric SCD first needs to be estimated and subtracted from the total SCD. For this purpose, a stratospheric AMF is often used together with an independent estimate of the stratospheric VCD (e.g. from a chemistry transport model, a climatology or independent measurements) $\left(\mathrm{SCD}_{\text {strat }}=\mathrm{VCD}_{\text {strat }} \cdot M_{\text {strat }}\right)$.

If the trace gas is optically thin, the total air mass factor can be written as the sum of the box-AMFs of each layer weighted by the partial vertical column (e.g., Palmer et al. (2001), Boersma et al. (2004)):

$$
M=\frac{\sum_{l} m_{l}(\widehat{\mathbf{b}}) x_{a, l}}{\sum_{l} x_{a, l}}
$$

In Eq. $3.2 m_{l}$ is the box-AMF and $x_{a, l}$ is the trace gas sub-column in layer $l$. However, as the actual profile of sub-columns is unknown, an a priori profile has to be used in the AMF calculation. The summation is done over the atmospheric layers $(l)$ of the a priori trace gas profile. In this step of the AMF calculation, apart from the profile shape of the trace gas, it is also necessary to have the best estimates for other forward model parameters $(\widehat{\mathbf{b}})$ such as satellite viewing geometry, surface pressure and surface reflectivity. Surface reflectivity depends on the surface properties and the geometry of the incident and reflected light. This anisotropy is described by the bidirectional reflectance distribution function (BRDF). In practice, surface reflectivity is often approximated by an isotropic Lambertian equivalent reflector (LER). There are different sources from which the a priori information can be obtained. It is desirable to use as many forward model parameters as possible retrieved from the satellite instrument itself. This practice gives consistency to the trace gas retrieval regarding the forward model parameters.

The $\mathrm{NO}_{2}$ and HCHO absorption cross sections used in the SCD fit and box-AMF calculation are representative for one fixed temperature. However, these cross sections vary with temperature, so it is necessary to apply a temperature correction. This correction accounts for the change in the absorption cross section spectrum at a specific layer as a function of the effective temperature (see Eq. S3.1 in the supplement), based on temperature and trace gas profiles from model data or climatologies. The correction is commonly 
done by applying a correction factor $\left(c_{l}\right)$ for each layer in the AMF calculation.

$$
M=\frac{\sum_{l} m_{l}(\widehat{\mathbf{b}}) x_{a, l} \cdot c_{l}}{\sum_{l} x_{a, l}}
$$

Most of the studies in which the temperature effect on the $\mathrm{NO}_{2}$ cross section is analyzed assume a simple dependency of the correction factor to temperature (Vandaele et al., 2002) (see Eq. S3.2 and S3.3 for typically used correction factors). For satellite applications, the change of the absorption cross section in case of $\mathrm{NO}_{2}$ has been reported to be approximately $-0.3 \%$ per $\mathrm{K}$ in the visible (Bucsela et al. (2013), Boersma et al. (2002)) and $-0.05 \%$ per K for HCHO (De Smedt, 2011).

Satellite retrievals also need to consider the presence of clouds. In the AMF calculation, residual clouds can be accounted for in several ways. The independent pixel approximation (IPA) consists of calculating the AMF for a partly cloudy scene as a linear combination of cloudy $\left(M_{c l}\right)$ and clear $\left(M_{c r}\right)$ components of the AMF, weighted by the cloud radiance fraction $w$ (i.e. the fraction of radiance that originates from the cloudy part of the pixel) (Martin et al. (2002), Boersma et al. (2004)):

$$
M=w M_{c l}+(1-w) M_{c r}
$$

In Eq. $3.4 w$ is wavelength dependent through radiation intensity, so it will be different for $\mathrm{NO}_{2}$ and $\mathrm{HCHO}$ (see Eq. S3.4 in the supplement).

AMFs for cloudy scenes are calculated using Eq. 3.3 with a specific cloud albedo and cloud pressure, with $m_{l}=0$ below the cloud. In line with assumptions made in current cloud retrievals, the cloud is considered as a Lambertian reflector with a fixed cloud albedo. This simple cloud model is in most cases suitable to be used in trace gas retrieval algorithms (Acarreta et al., 2004). As an alternative, the radiative effects of the cloudy parts of the pixels can be calculated by representing the clouds as volume scatterers (see Sect. S1.3), or the cloud structures and their radiative properties can be simulated using 3D RTMs (e.g. O'Hirok and Gautier (1998)).

The atmosphere can also be assumed to be cloud-free for cloud fractions below a certain threshold (e.g. 0.1 or 0.2 , see Table 3.3). In that case, a clear-sky AMF is used and Eq. 3.4 reduces to $M=M_{c r}$. For cloud fractions larger than the clear-sky threshold but below a cloudy-sky threshold, IPA is sometimes applied. Generally, measurements with cloud fractions higher than the cloudy-sky threshold are discarded or flagged. There is also the possibility to account for cloud-aerosol mixtures; in this case the threshold for using either clear-sky AMF or IPA can depend on both cloud fraction and cloud altitude (see Sect. S1.3). In all approaches accurate information is needed on the cloud radiance 
fraction and in the cloud height.

Different retrieval groups use different sources for the ancillary data, as well as different methods to account for the temperature dependence and the presence of clouds and aerosols (e.g. Van Noije et al. (2006)). In our study, each of the groups first calculated tropospheric AMFs using harmonized settings, i.e. using the same forward model parameters, temperature correction and cloud correction. In order to calculate the total AMF using Eq. 3.3, an interpolation from the LUT needs to be done to obtain the box-AMFs at the specific values of the forward model parameters. Furthermore, a vertical interpolation is required to adjust the vertical discretization of the a priori absorber profile to the one of the LUT. From the comparison of the tropospheric AMFs calculated using harmonized settings, we can thus obtain a relative AMF structural uncertainty, which is determined by different approaches in interpolation and vertical discretization of the box-AMFs, assuming that the selected forward model parameters are the true values.

Next, each of the groups used their preferred settings to calculate tropospheric AMFs. In this round-robin exercise, a comparison of state-of-the-art retrieval algorithms, the differences between AMFs not only arise from differences between the RTMs, vertical discretization and interpolation but also from differences in the selection of forward model parameter values and the different corrections for clouds, aerosols and surface reflectivity. Thus the differences in the AMFs using preferred settings can be interpreted as the overall structural uncertainty of the AMF calculation (Thorne et al., 2005).

\subsubsection{Participating models}

Four RTMs from different research groups participated in the comparison. Some differences between models are highlighted in Table 3.1. A brief summary for each model is listed alphabetically in this section and more detailed information about the models can be found in the references.

\section{DAK}

DAK (Doubling-Adding KNMI) was developed at the Royal Netherlands Meteorological Institute (Stammes, 2001). DAK uses the doubling-adding method for solving the radiative transfer equation (Stammes et al. (1989), de Haan et al. (1987)). The method consists of first calculating the reflection and transmission properties of a homogeneous layer by repeated doubling, starting with a very thin layer, and then adding homogeneous layers on top of each other, which then yields the reflection and transmission of the combined layers. The internal radiation field is computed at the interface of all layers and the radiation emerging at the top of the atmosphere and at the surface is calculated. 
Table 3.1: Overview of radiative transfer models that participated in the top-of-atmosphere reflectance comparison and their main characteristics.

\begin{tabular}{lllll}
\hline Model & DAK & McArtim & SCIATRAN & VLIDORT \\
\hline Reference & Stammes (2001) & $\begin{array}{l}\text { Deutschmann et al. } \\
(2011)\end{array}$ & Rozanov et al. (2014) & Spurr et al. (2001) \\
\hline Institute & KNMI, WUR & MPI-C & IUP-UB & IASB-BIRA \\
\hline $\begin{array}{l}\text { Solving the } \\
\begin{array}{l}\text { Radiative } \\
\text { Transfer } \\
\text { equation }\end{array}\end{array}$ & $\begin{array}{l}\text { Doubling adding } \\
\text { method }\end{array}$ & $\begin{array}{l}\text { Monte Carlo methods } \\
\text { to solve integral } \\
\text { form of RTE }\end{array}$ & $\begin{array}{l}\text { Source function } \\
\text { integration technique } \\
\text { and discrete - } \\
\text { ordinate method }\end{array}$ & $\begin{array}{l}\text { Linearized discrete } \\
\text { ordinate solution }\end{array}$ \\
$\begin{array}{l}\text { Sphericity } \\
\text { correction }\end{array}$ & $\begin{array}{l}\text { Pseudo spherical } \\
\text { for direct solar } \\
\text { incident photons }\end{array}$ & $\begin{array}{l}\text { Full 3D spherical model } \\
\text { calculations on a sphere }\end{array}$ & $\begin{array}{l}\text { Full spherical mode } \\
\text { for solar and single } \\
\text { scattered photons }\end{array}$ & $\begin{array}{l}\text { Pseudo spherical } \\
\text { for solar and single } \\
\text { scattered photons }\end{array}$ \\
\hline
\end{tabular}

DAK accounts for multiple scattering and polarization. It is also possible to account for Earth's sphericity using the pseudo spherical option, which corrects for sphericity in the light path of the direct solar beam, but not in the scattered beam.

Box-AMFs are calculated with DAK in this study by WUR/KNMI by differencing the logarithm of reflectances at TOA with and without the trace gas in atmospheric layer $l$ divided by the gas absorption optical thickness of the layer $\tau_{\text {gas }}$ :

$$
m_{l}=-\frac{\ln R\left(\tau_{\text {gas }, l}\right)-\ln R\left(\tau_{\text {gas }, l}=0\right)}{\tau_{\text {gas }, l}}
$$

\section{McArtim}

McArtim (Monte Carlo Atmospheric Radiative Transfer Inversion Model) (Deutschmann et al., 2011) was developed at University of Heidelberg and Max-Planck Institute for Chemistry (MPI-C, Mainz). It is based on the backward Monte Carlo method: a photon emerges from a detector in an arbitrary line of-sight direction and is followed in the backward direction along the path until the photon leaves the top of the atmosphere. The various events which may happen to the photon at various altitudes are defined by suitable probability distributions. At each scattering event the probability that the photon is scattered into the direction of the Sun is calculated and the intensity of the photon is weighted by the sum of the probabilities of all scattering events (local estimation method). In this RTM, the integro-differential equation for radiative transfer is deduced and solved using Neumann series, the summands of which are linked with the contributions of multiple scattering orders to the radiation field. McArtim is a 3D-model and uses full spherical geometry, which means that sphericity is accounted for incoming, single scattered and multiple scattered photons. The model is capable of including polarization and rotational Raman scattering (which are included in the simulations shown in this 
study).

Box-AMFs calculated by MPI-C are obtained from Jacobians (derived by $W=\frac{\partial \ln I}{\partial \beta}$, with $\beta\left(\mathrm{km}^{-1}\right)$ the absorption coefficient) for each grid box according to the formula:

$$
m_{l}=-\frac{W}{I \Delta h}
$$

In Eq. $3.6 W$ refers to the Jacobian $(\mathrm{km}), I$ is the simulated radiance at TOA normalized by the solar spectrum (unitless) and $\Delta h$ is the grid box thickness $(\mathrm{km})$.

\section{SCIATRAN}

SCIATRAN (Rozanov et al., 2014) was developed at the Institute of Environmental Physics at the University of Bremen (IUP-UB) in Germany. It models radiative transfer processes in the atmosphere from the UV to the thermal infrared, in both scalar and vector mode, i.e. with the option to account for polarization. The simulations can be done for a plane parallel, pseudo-spherical or fully spherical atmosphere. In the fully spherical approach, the integral radiative transfer equation is solved accounting for single scattering in spherical mode, and multiple scattering is approximated with a solution of the differential-integral radiative transfer equation in the plane parallel mode.

SCIATRAN calculates the Jacobians or weighting functions, which are the derivatives of the simulated radiance with respect to atmospheric and surface parameters (air number density in this case). These quantities are related to the box-AMFs calculated by IUP-UB as follows:

$$
m_{l}=-\frac{W_{l}}{I \sigma \Delta h_{l}}
$$

$W_{l}\left(\mathrm{~W} \cdot \mathrm{m}^{-2} \cdot \mathrm{nm}^{-2} \cdot \mathrm{sr}^{-1} / \mathrm{molec} \cdot \mathrm{cm}^{-3}\right)$ is the weighting function at atmospheric level $l, I$ $\left(\mathrm{W} \cdot \mathrm{m}^{-2} \cdot \mathrm{nm}^{-2} \cdot \mathrm{sr}^{-1}\right)$ is the TOA radiance, $\sigma_{l}\left(\mathrm{~cm}^{2} / \mathrm{molec}\right)$ is the absorber cross section and $\Delta h_{l}(\mathrm{~cm})$ is the thickness of the layer.

\section{VLIDORT}

VLIDORT (Vector-LInearized Discrete Ordinate Radiative Transfer) was developed by Rob Spurr at RT SOLUTIONS, Inc. The model is based on the discrete ordinate approach to solve the radiative transfer equation in a multi-layered atmosphere, reducing the RTE to a set of coupled linear first order differential equations. Then, perturbation theory is applied to the discrete ordinate solution (Spurr et al., 2001). Intensity and partial derivatives of intensity with respect to atmospheric parameters and surface parameters (i.e. weighting functions) are determined for upwelling direction at TOA, for 
arbitrary angular direction. The pseudo spherical formulation in VLIDORT corrects for the curved atmosphere in the solar and scattered beam (for single scattering, not for multiple scattering).

Box-AMFs are derived from the altitude-dependent weighting functions determined by VLIDORT:

$$
m_{l}=\frac{\partial \ln I}{\partial \tau_{\text {gas }, l}}=\left(\tau_{\text {gas }, l} \cdot \frac{\partial I}{\partial \tau_{\text {gas }, l}}\right) /\left(I \cdot \tau_{\text {gas }, l}\right)
$$

$I\left(\mathrm{~W} \cdot \mathrm{m}^{-2} \cdot \mathrm{nm}^{-2} \cdot \mathrm{sr}^{-1}\right)$ is the TOA radiance, $\tau_{\text {gas }}$ is the trace gas absorption optical thickness of the layer and the term $\left(\tau_{g a s, l} \cdot \frac{\partial I}{\partial \tau_{\text {gas }, l}}\right)$ is the altitude dependent weighting function.

\subsection{Results}

\subsubsection{TOA reflectances}

As a first exercise, a base case calculation and comparison of TOA reflectances was made to assess the performance of the four RTMs and to obtain the structural uncertainty in TOA reflectance modelling. The base case comparison allowed us to establish the best possible level of agreement between RTMs by identifying differences in the RTMs performance that in more complex settings would be difficult to recognize. Furthermore, total and ozone optical thickness were compared to evaluate how the models agreed in their treatment of scattering and absorption processes and whether differences in scattering and absorption can explain possible differences between the TOA reflectances.

Basic model parameters were established as input in all RTMs (details can be found in Table S3.1 in the supplementary material). The basic atmospheric profile was a 33-layer mid-latitude summer atmosphere (Anderson et al., 1986), and every group performed their own vertical discretization of this profile.

In the RT modelling, we considered a clear sky atmosphere, so clouds and aerosols were not included. Rayleigh scattering and $\mathrm{O}_{3}$ absorption were included, but Raman scattering was not included. The temperature dependence of the ozone cross-section was neglected in the reflectance calculation. TOA reflectances were calculated at 7 wavelengths, including 440 and $340 \mathrm{~nm}$ which are relevant for the retrievals of $\mathrm{NO}_{2}$ and $\mathrm{HCHO}$, respectively. Both scalar (i.e. without polarization) and vector (i.e. with polarization) calculations were performed in most of the cases. All models applied their particular sphericity treatments to the calculations. The surface was considered as a Lambertian reflector by all the RTMs. This approximation assumes that surface reflectivity is isotropic (i.e it does not consider 


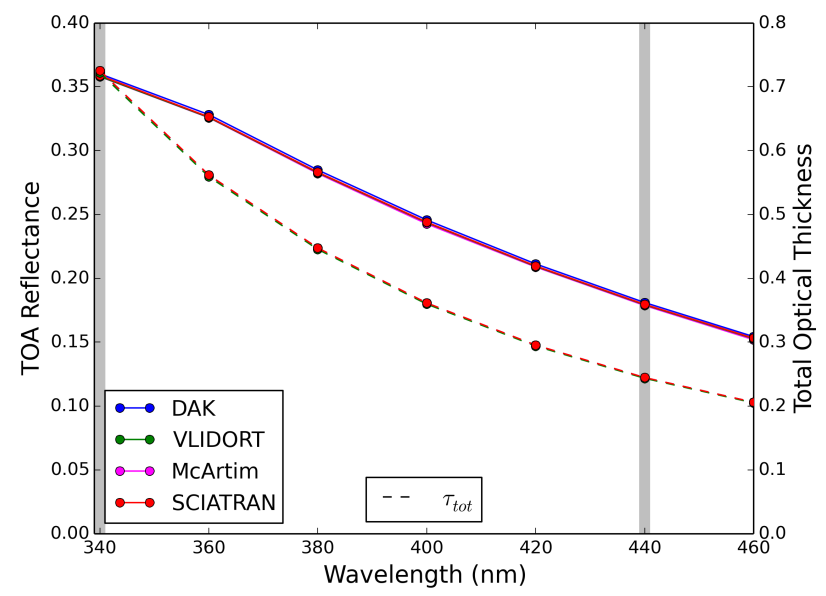

Figure 3.2: TOA reflectances simulated by 4 RTMs for $\theta_{0}=37^{\circ}\left(\mu_{0}=0.8\right)$, off-nadir viewing angle $\theta=72.5^{\circ}(\mu=0.3)$ and $\varphi=0^{\circ}$ as a function of wavelength (in $20 \mathrm{~nm}$ steps). Dashed lines represent total optical thickness computed by each RTM. Grey bands indicate the relevant wavelengths for $\mathrm{HCHO}(340 \mathrm{~nm})$ and $\mathrm{NO}_{2}(440 \mathrm{~nm})$. Surface albedo is 0 and surface pressure is $1013 \mathrm{hPa}$.

the directionality of the surface reflectance distribution). The selected geometries covered a wide range of values for solar zenith angle (SZA, $\theta_{0}$ ), viewing zenith angle (VZA, $\theta$ ), and relative azimuth angle (RAA, $\varphi=180^{\circ}-\left|\phi-\phi_{0}\right|$, where $\phi-\phi_{0}$ is the viewing direction minus solar direction). All the angles are specified with respect to the surface. The values for SZA span the typical range of what UV/Vis sensors are encountering in orbit, and the maximum value of VZA is related to the higher possible values of this parameter for the future TROPOMI instrument (72.5) (van Geffen et al., 2018).

All models calculate the same spectral dependency of TOA reflectance, as shown in Fig. 3.2 (solid line). TOA reflectance increases towards shorter wavelengths due to stronger Rayleigh scattering. TOA reflectance simulated by the different models agree within $1.3 \%$ for the geometries included in Fig. 3.2. The dashed line in Fig. 3.2 shows the total optical thickness as a function of wavelength for DAK, SCIATRAN and VLIDORT (McArtim does not provide this output), and is generally consistent within $0.15 \%$ for all wavelengths except $340 \mathrm{~nm}$, where the differences are $0.5 \%$.

Figure 3.3 shows the distribution of relative differences (defined as $(100(a-b) / a)$ ) between TOA reflectances simulated by the four RTMs at $340 \mathrm{~nm}$ and $440 \mathrm{~nm}$. The distribution is determined by the relative differences between all combinations of model differences, including all simulated geometry scenarios for a surface albedo of 0 and terrain pressure of $1013 \mathrm{hPa}$. According to the standard deviation in both distributions (dashed lines in Fig. 3.3), the relative differences are below $1.5 \%$ at $340 \mathrm{~nm}$ and $1.1 \%$ at 440 $\mathrm{nm}$ in most geometry configurations ( $80 \%$ of the samples of the distribution), including the most common retrieval scenarios. The tails of the distributions at both wavelengths 

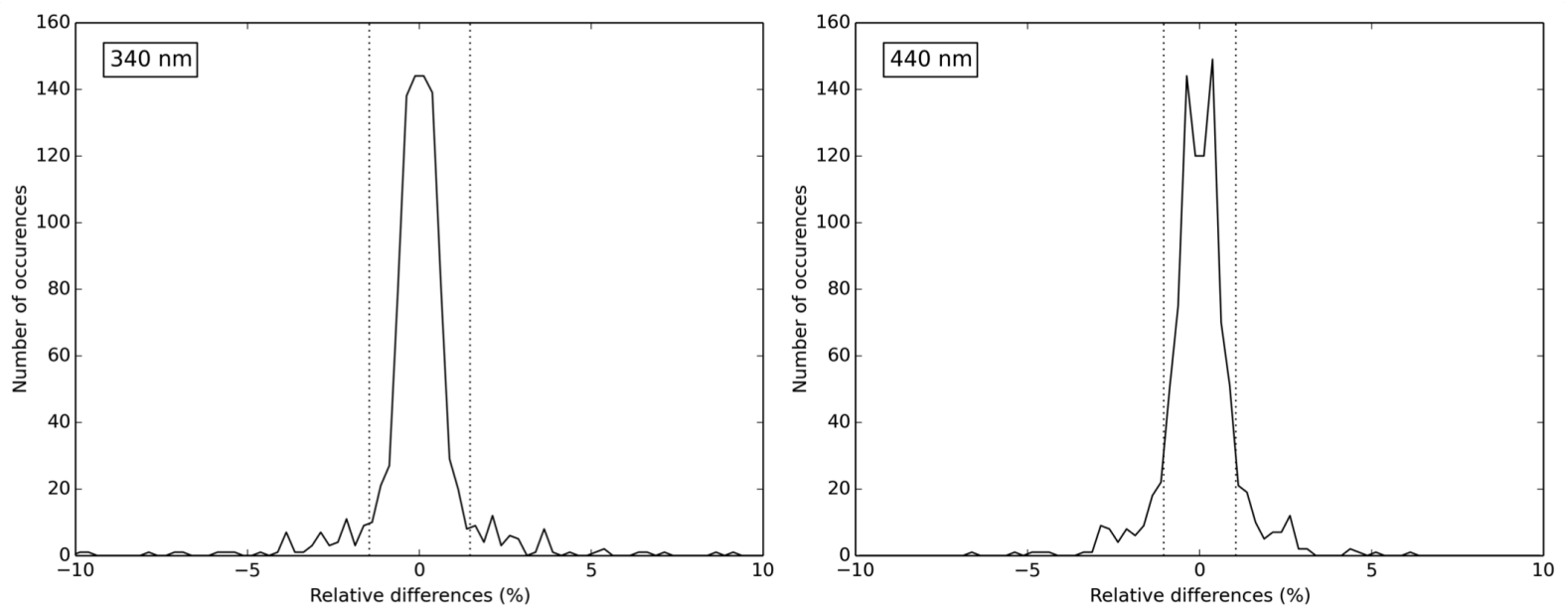

Figure 3.3: Distribution of relative model differences between TOA reflectances simulated by four RTMs including polarization (DAK-VLIDORT, DAK-SCIATRAN, DAK-McArtim, VLIDORT-SCIATRAN, VLIDORT-McArtim, SCIATRAN-VLIDORT and reversed combinations) for all geometry combinations $\left(0^{\circ}<\theta_{0}<90^{\circ}, \theta=0^{\circ}, 72.5^{\circ}\right.$ and $\varphi=0^{\circ}, 60^{\circ}, 90^{\circ}$, $120^{\circ}, 180^{\circ}$ ) (see Table S3.1 for exact values) at $340 \mathrm{~nm}$ (left panel) and $440 \mathrm{~nm}$ (right panel). The dashed lines represent the median plus/minus the standard deviation of the distribution. Surface albedo is 0 and surface pressure is $1013 \mathrm{hPa}$. Sample size in each distribution is 960 .

correspond to extreme viewing geometries, i.e. for scenarios in which solar and viewing zenith angles are both large. Mean relative differences over all RTM pairs are at most $6.4 \%$ for extreme geometries $\left(\theta_{0}=87^{\circ}, \theta=72.5^{\circ}\right)$, and for shorter wavelengths. For nadir view $\left(\theta=0^{\circ}\right)$ relative differences are on average two times smaller than for larger VZA $\left(\theta \geq 60^{\circ}\right)$ at both 340 and $440 \mathrm{~nm}$.

The results show strong consistency of TOA reflectance calculations for the most common moderate viewing geometry retrieval scenarios. Relative differences are somewhat higher for larger VZA, SZA and shorter wavelengths. For the more extreme geometries, the light path through the atmosphere is generally longer and photons have higher probability of undergoing interactions (scattering, absorption) with the atmosphere. Furthermore, differences in the treatment of Earth's sphericity for the extreme geometries have a stronger influence than in close to nadir viewing geometries. These differences will still be present in the box-AMFs comparison in Sect. 3.3.2. Rayleigh scattering also affects the effective photon path and it is stronger at $340 \mathrm{~nm}$ than at $440 \mathrm{~nm}$. Thus, small differences in the description of Rayleigh scattering in the RTMs are more likely to lead to differences for the extreme geometries and shorter wavelengths. The standard deviation of differences between modelled TOA reflectances of 1.5\% (at $340 \mathrm{~nm}$ ) and $1.1 \%$ (at $440 \mathrm{~nm}$ ) in this comparison can be considered as the reflectance structural uncertainty. The agreement in this study is better than in previous RTM comparisons like Wagner et al. (2007) and Stammes (2001) which reported differences of 5\%. The detailed RTM comparison will serve as a test bed to analyze the performance of other RTMs. 

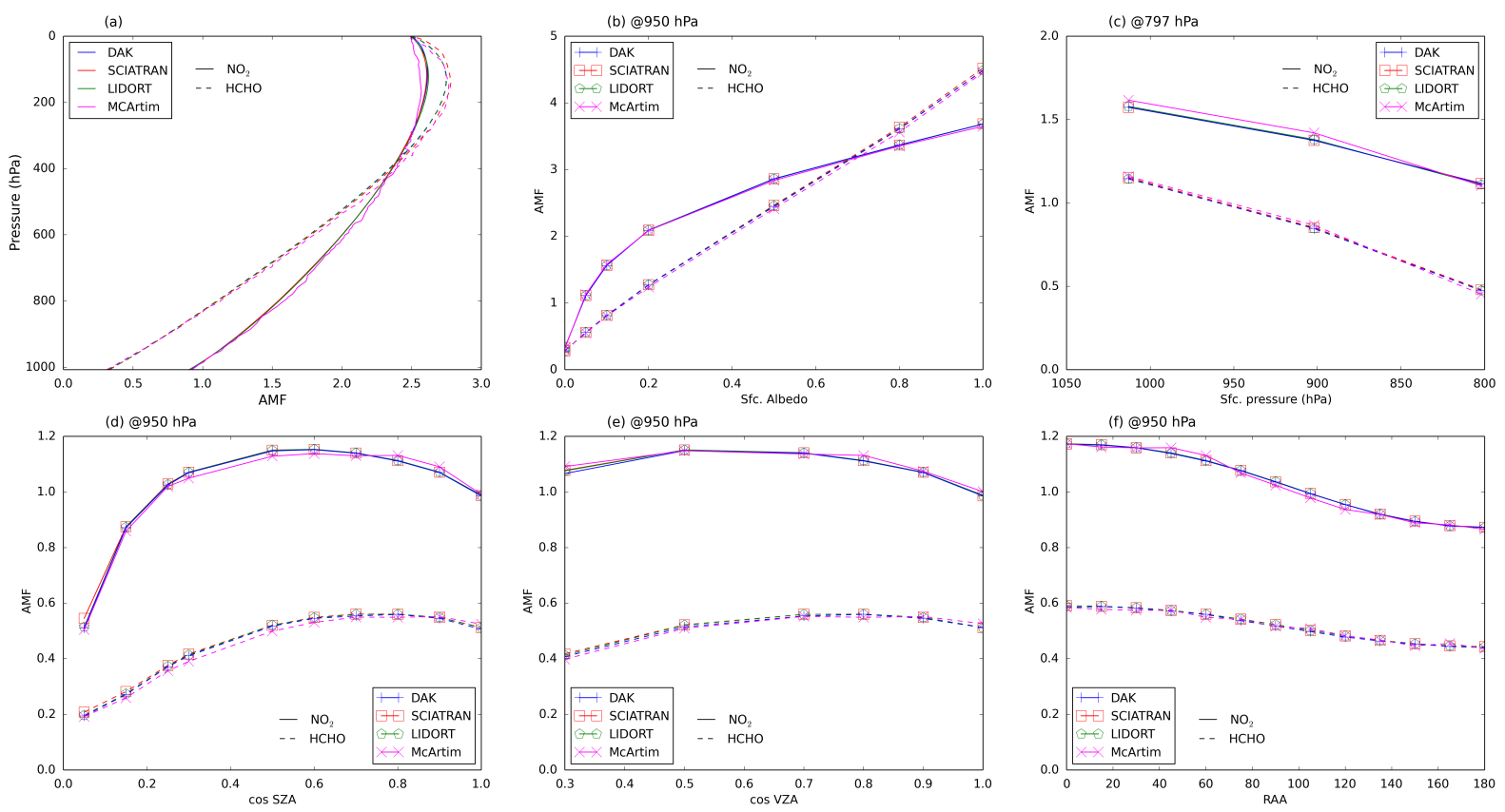

Figure 3.4: Box-AMF dependencies on forward model parameters for $\mathrm{NO}_{2}$ at $440 \mathrm{~nm}$ (solid lines, circles) and $\mathrm{HCHO}$ at $338 \mathrm{~nm}$ (dashed lines, triangles) for a clear-sky atmosphere. (a) Box-AMFs vertical profile; (b) $950 \mathrm{hPa}$ box-AMF as a function of surface albedo; (c) $797 \mathrm{hPa}$ box-AMF as a function of surface pressure; (d) $950 \mathrm{hPa}$ box-AMF as a function of cosine of SZA, (e) $950 \mathrm{hPa}$ box-AMF as a function of cosine of VZA, (f) $950 \mathrm{hPa}$ box-AMF as a function of RAA. In all panels the fixed parameters are: $\mu_{0}=\mu=0.8\left(\theta_{0}=\theta=37^{\circ}\right), \varphi=60^{\circ}$, surface albedo $=0.05$, surface pressure $=1013 \mathrm{hPa}$.

\subsection{2 $\mathrm{NO}_{2}$ and $\mathrm{HCHO}$ altitude-dependent (box-) air mass factors}

To calculate box-AMFs, a common vertical grid was agreed between the groups in order to reduce the sources that might cause differences between the RTMs. The common profile resolution was $0.1 \mathrm{~km}$ from the surface up to $10 \mathrm{~km}, 1 \mathrm{~km}$ resolution from 10 to $60 \mathrm{~km}$ and $2 \mathrm{~km}$ resolution from 60 to $100 \mathrm{~km}$. $\mathrm{NO}_{2}$ box-AMFs were calculated at $440 \mathrm{~nm}$ and HCHO box-AMFs were calculated at 338, 341 and $344 \mathrm{~nm}$ to investigate the wavelength dependency (not shown). Box-AMFs were calculated accounting for polarization of light and Earth's sphericity. The number of reference points for surface albedo was increased and several surface pressures were added relative to the TOA reflectance simulations in the previous section to cover a wider range of scenarios. All settings are detailed in Table S3.2.

Figure 3.4(a) shows that the 4 participating groups generally agree well on the vertical profile shape of $\mathrm{NO}_{2}$ and $\mathrm{HCHO}$ box-AMFs in the troposphere. Measurement sensitivity decreases towards the surface, due to the increase of light scattering in the lower troposphere. Measurement sensitivity to $\mathrm{HCHO}$ is substantially lower than to $\mathrm{NO}_{2}$, because of stronger Rayleigh scattering at shorter wavelengths. McArtim box-AMFs have lower 
values in the stratosphere (pink line), presumably reflecting the more realistic description of atmosphere's sphericity in McArtim relative to the other models (see Sect. 3.3.4 for specific sphericity effect on AMFs). The vertical profile of McArtim shows a wavering line due to the statistical noise in the Monte Carlo simulations (which can be reduced by increasing the number of simulations). Figure 3.4 (b), (d)-(f) shows the $\mathrm{NO}_{2}$ and $\mathrm{HCHO}$ box-AMF dependency on forward model parameters (surface albedo, surface pressure, SZA, VZA and RAA) in the lower troposphere at $950 \mathrm{hPa}$. This pressure level (close to the surface) is especially relevant because this is where trace gas concentrations are enhanced in polluted situations. The sensitivity to surface albedo at $950 \mathrm{hPa}$ (Fig. 3.4b) is similar for all four RTMs. Box-AMFs increase with surface albedo due to a stronger reflection of light at the surface. This increase is particularly strong for low values of surface albedo. For an albedo of 0.05 , an increase of 0.01 in the surface albedo results in an increase of $11 \%$ in the $\mathrm{NO}_{2}$ box-AMF at $440 \mathrm{~nm}$ and of $9 \%$ in the case of HCHO at 338 $\mathrm{nm}$. The increase in the box-AMFs is less steep for higher values of surface albedo. Thus, an accurate knowledge of surface albedo is required especially for low albedo values. For surface pressure (Fig. 3.4c), the box-AMF (at $797 \mathrm{hPa}$ ) decreases with decreasing surface pressure. For increasing terrain height, the amount of light scattered and reflected from below $797 \mathrm{hPa}$ decreases. In a more elevated terrain, the photons undergo fewer scattering events, which tends to reduce box-AMFs at a specific level. Models agree well in representing this sensitivity. An error in the surface pressure of $10 \mathrm{hPa}$ leads to $\pm 2 \%$ errors in the lower tropospheric box-AMF values, which indicates the importance of accurate surface pressure information that is representative for the entire pixel area. Box-AMFs at $950 \mathrm{hPa}$ show relatively weak dependency on VZA (Fig. 3.4e) and RAA (Fig. 3.4f) and stronger dependency on high values of SZA (Fig. 3.4d), but all RTMs agree well on measurement sensitivity to geometry parameters.

Figure 3.5 shows the vertical profile of mean relative differences in $\mathrm{NO}_{2}$ (left panel) and $\mathrm{HCHO}$ (right panel) box-AMFs between all the models, for a specific surface albedo and surface height and a wide range of solar and viewing geometries. Generally, models reproduce box-AMFs to within $2 \%$ for $\mathrm{NO}_{2}$ and $2.6 \%$ for HCHO. Mean relative differences are higher at the lowest layers and around $300 \mathrm{hPa}$. This is due to unavoidable slight differences in vertical discretization of the surface-atmosphere boundary and where the resolution changes from 0.1 to $1 \mathrm{~km}$ at $10 \mathrm{~km}$ altitude in the different models. Specific differences were also found in the mid-upper troposphere and stratosphere, where McArtim is on average lower than the other RTMs. Those differences illustrate the different treatment of multiple scattering within the models. McArtim accounts for multiple scattering in a fully spherical atmosphere, whereas DAK, VLIDORT and SCIATRAN simulate multiple scattering in a plane parallel atmosphere. In a spherical atmosphere, less light is horizontally scattered into the line of sight of the instrument than in a plane parallel atmosphere (see Fig. S3.2), which is one of the reasons for lower box-AMFs by 
McArtim in the stratosphere (visible in Fig. 3.4a between 200-0 hPa).
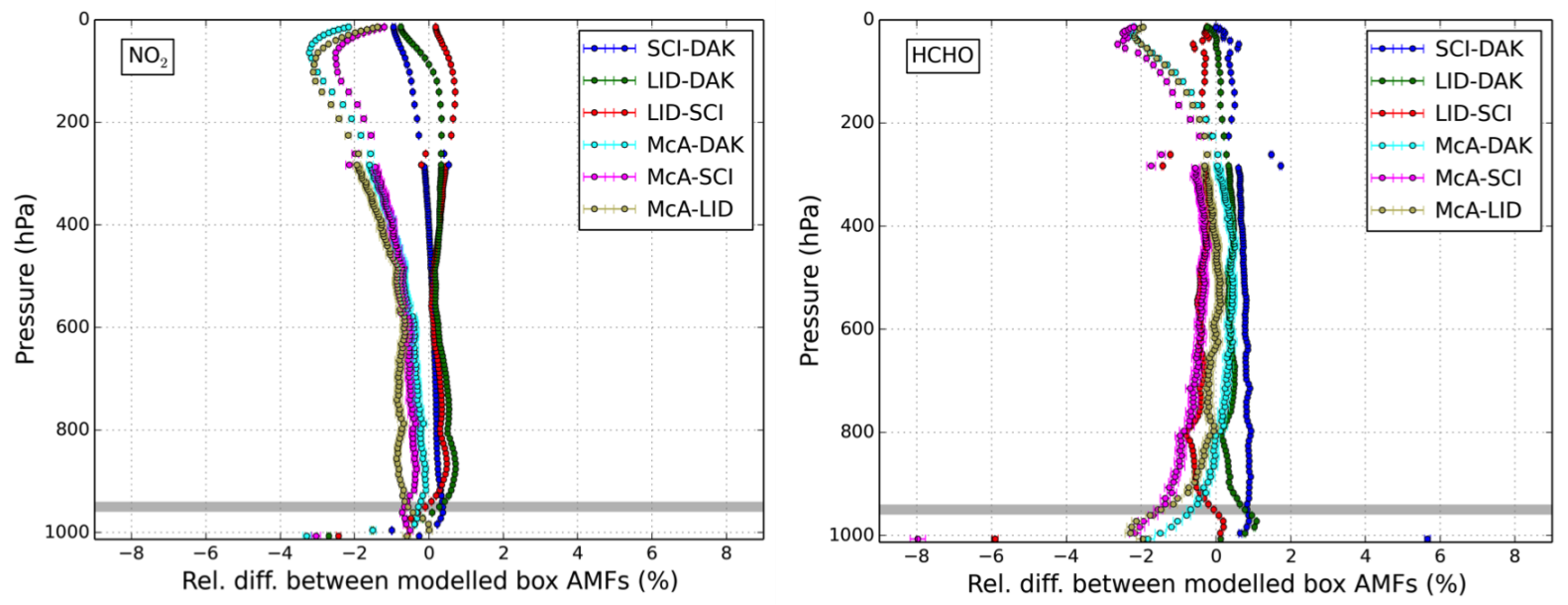

Figure 3.5: Vertical profile of mean relative differences between $\mathrm{NO}_{2}$ box-AMFs (left) and HCHO box-AMFs (right) from DAK, McArtim, SCIATRAN and VLIDORT for a wide range of satellite viewing geometry $\left(0^{\circ}<\theta_{0}<75^{\circ}, 0^{\circ}<\theta<72.5^{\circ}, 0^{\circ}<\varphi<180^{\circ}\right)$, surface albedo $=0.05$ and surface height $1013 \mathrm{hPa}$. Grey bands indicate $950 \mathrm{hPa}$ atmospheric layer.

Relative differences for $950 \mathrm{hPa}$ box-AMFs are below $1.1 \%$ for $\mathrm{NO}_{2}$ and below $2.6 \%$ for $\mathrm{HCHO}$ in most geometry configurations (according to the standard deviation of relative differences distribution for $950 \mathrm{hPa}$ box-AMFs, not shown). Higher relative differences mainly occur between McArtim and the other models. The highest relative differences occur for scenarios with high VZAs $\left(\theta=72.5^{\circ}\right.$ ) (not shown), again indicating that different Rayleigh scattering description and sphericity treatments in the radiative transfer modelling of the atmosphere are important.

This comparison indicates a good agreement between box-AMF LUTs computed using different RTMs. The structural uncertainty in the AMF calculation due to the choice of RTM and different interpolation schemes is $2 \%$ for $\mathrm{NO}_{2}$ and $2.6 \%$ for $\mathrm{HCHO}$. These results suggest that a correct treatment of the processes affecting the effective light path in the atmosphere is important for box-AMF calculation. The vertical discretization is also relevant in box-AMF calculations, as demonstrated by the differences at specific altitudes (Fig. 3.5) and by the box-AMF sensitivity to altitude (Fig. 3.4a). Therefore, the vertical sampling of the LUT should have a fine resolution, especially in the lower troposphere where strong gradients in $\mathrm{NO}_{2}$ and $\mathrm{HCHO}$ concentrations occur. The dependencies of the box-AMFs at low surface albedo values (Fig. 3.4b) and to surface pressure (Fig. 3.4c), suggest that the number of reference points in the LUT for these parameters should be large. 


\subsubsection{Tropospheric air mass factors}

In order to compute tropospheric AMFs via Eq. 3.3 we need to interpolate the boxAMFs from the LUT for the best estimate of the forward model parameters $\mathbf{b}$. Generally a 6-D linear interpolation (or 5-D if the vertical resolution of the LUT and the a priori profile vertical grid are equal) is done over all the parameters on which the box-AMF depend. For each dimension, the two closest values to the exact pixel parameters are used to obtain the interpolated box-AMF $\left(m_{l}\right.$ in Eq. 3.3). This approach will introduce systematic errors in case of nonlinear dependencies of the parameters in the LUT. Pixelby-pixel online calculations of box-AMFs would avoid interpolation errors; Castellanos et al. (2015) estimated the differences between on-line and LUT-derived AMFs to be on average less than $1 \%$, for individual measurements less than $8 \%$, with an upper bound of the difference of $20 \%$ over South America. Lin et al. (2014) found 1-5\% differences on retrieved VCDs with and without LUT over China.

\section{Harmonized settings}

Four groups used the same settings (forward model parameters, a priori profiles, temperature and cloud correction) to calculate clear sky and total tropospheric $\mathrm{NO}_{2} \mathrm{AMFs}$ for one specific OMI orbit over Australia and Eastern Asia on 02 February 2005 (See Fig. 3.6). The selected harmonized settings were those from KNMI/WUR (see Table 3.3). All groups applied the same temperature correction (from Boersma et al. (2004), (see Eq. S3.1)) and cloud correction via the independent pixel approximation. The aim of this comparison was to obtain an estimate of the structural AMF uncertainty introduced by different vertical discretization and the interpolation schemes assuming that the values of the selected forward model parameters are true.

All groups calculate similar AMF spatial patterns for the selected orbit. Figure 3.6 (upper panels) shows total tropospheric $\mathrm{NO}_{2}$ AMFs calculated by each group. The distribution of the AMF values along the orbit is determined by the different parameters on which AMFs depend. Lower panels in Fig. 3.6 show $\mathrm{NO}_{2}$ (a priori) model vertical column, surface albedo and cloud fraction in the orbit. At high latitudes, where surface albedo is high, AMFs are up to 3-5. Surfaces with high albedo (usually covered by snow or ice) reflect more radiation than surfaces with lower surface albedo, and this increases the AMF values. The effect of clouds and the a priori profile is also visible: AMFs are generally low in cloudy regions and over polluted regions in east China $\left(\sim 30^{\circ} \mathrm{N}\right)$, indicative of reduced sensitivity to $\mathrm{NO}_{2}$ in the lowest layers of the atmosphere.

The correlation between AMFs calculated by the different retrieval groups is excellent $\left(\mathrm{R}^{2}>0.99\right)$. Overall, tropospheric AMFs agree within $6.5 \%$ in polluted areas and within 
Table 3.2: Statistical parameters for the comparison of total tropospheric $\mathrm{NO}_{2}$ AMFs for polluted and unpolluted pixels (pixels with model $\mathrm{NO}_{2}$ vertical column higher or lower than $1 \cdot 10^{15} \mathrm{molec} / \mathrm{cm}^{2}$ respectively) between the different retrieval groups for one complete orbit from 02 February 2005 (2005m0202t0339-o02940 v003). Only pixels with effective (i.e. radiometrically equivalent) cloud fraction $\leq 0.2$ are considered. Mean, median and sigma are relative differences in $\%(100(a-b) / a)$.

\begin{tabular}{ccccccc}
\hline \multicolumn{7}{c}{ Polluted pixels (\#1983) } \\
\hline $\begin{array}{c}\text { Diff. } \\
\text { between }\end{array}$ & $\begin{array}{c}\text { Mean } \\
\text { (rel. diff.) }\end{array}$ & $\begin{array}{c}\text { Median } \\
\text { (rel. diff.) }\end{array}$ & $\begin{array}{c}\sigma \\
\text { (rel. diff.) }\end{array}$ & $\mathrm{R}^{2}$ & Slope & Offset \\
\hline IUP-WUR & $-3.8 \pm 0.3$ & -2.5 & 6.4 & 0.9968 & 0.96 & 0.08 \\
\hline BIRA-WUR & $0.5 \pm 0.02$ & 0.5 & 0.8 & 0.9996 & 0.98 & 0.02 \\
\hline BIRA-IUP & $3.9 \pm 0.7$ & 2.9 & 4.8 & 0.9967 & 1.02 & -0.07 \\
\hline MPIC-WUR & $-1.5 \pm 0.1$ & -0.9 & 4.7 & 0.9957 & 0.99 & 0.03 \\
\hline MPIC-IUP & $2.1 \pm 0.9$ & 0.5 & 4.9 & 0.9955 & 1.03 & -0.06 \\
\hline MPIC-BIRA & $-2.0 \pm 0.1$ & -1.2 & 4.7 & 0.9957 & 1.01 & 0.01 \\
\hline \hline \multicolumn{7}{c}{ Unpolluted pixels $(\# 23744)$} \\
\hline IUP-WUR & $-0.4 \pm-0.3$ & -0.3 & 2.4 & 0.9983 & 0.96 & 0.06 \\
\hline BIRA-WUR & $0.6 \pm 0.004$ & 0.3 & 0.8 & 0.9995 & 0.98 & 0.03 \\
\hline BIRA-IUP & $1.0 \pm 0.04$ & 0.7 & 1.9 & 0.9989 & 1.01 & -0.04 \\
\hline MPIC-WUR & $-0.5 \pm 0.02$ & -0.4 & 2.1 & 0.9985 & 0.97 & 0.06 \\
\hline MPIC-IUP & $-0.1 \pm 0.06$ & -0.4 & 2.2 & 0.9981 & 1.01 & -0.01 \\
\hline MPIC-BIRA & $-1.1 \pm 0.02$ & -0.9 & 1.7 & 0.9990 & 0.99 & 0.03 \\
\hline
\end{tabular}

$2.5 \%$ in clean remote areas for most retrieval scenarios, in line with the results from the box-AMF LUT comparison. BIRA AMFs are on average higher than AMFs by the other groups, generally by a few percent, and IUP-UB AMFs are on average lower for polluted and unpolluted situations. Table 3.2 summarizes the results of the comparison.

Largest differences are found at the edges of the OMI orbit, where viewing zenith angles are large and light paths are long. This can be seen in the lower right panel of Fig. 3.6, where the relative differences of tropospheric $\mathrm{NO}_{2}$ AMFs between MPI-C and WUR are clearly visible at the edges of the orbit. These differences are consistent with the higher sensitivity to tropospheric trace gases for extreme viewing zenith angles (also shown in Fig. 3.4e) in McArtim compared to DAK. Figure S3.1 in the supplementary material shows the relative differences between all AMFs calculated by the groups. Relative difference distributions show patterns that reflect the spatial distribution of surface albedo, clouds and $\mathrm{NO}_{2}$ (e.g. over southeastern Australia, East China and Korea). Large differences between the groups are found in cloudy situations. These effects reflect the uncertainties arising from the use of different RTM as well as from the interpolation and the vertical discretization of the LUT when calculating the AMFs.

These results demonstrate that even when similar RTMs, box-AMFs, and identical forward model parameters are used to calculate the AMFs, there is structural uncertainty that is introduced by the specific implementation of different groups. First, the choice of a RTM introduces uncertainty in the box-AMF calculation. Second, there are interpolation 

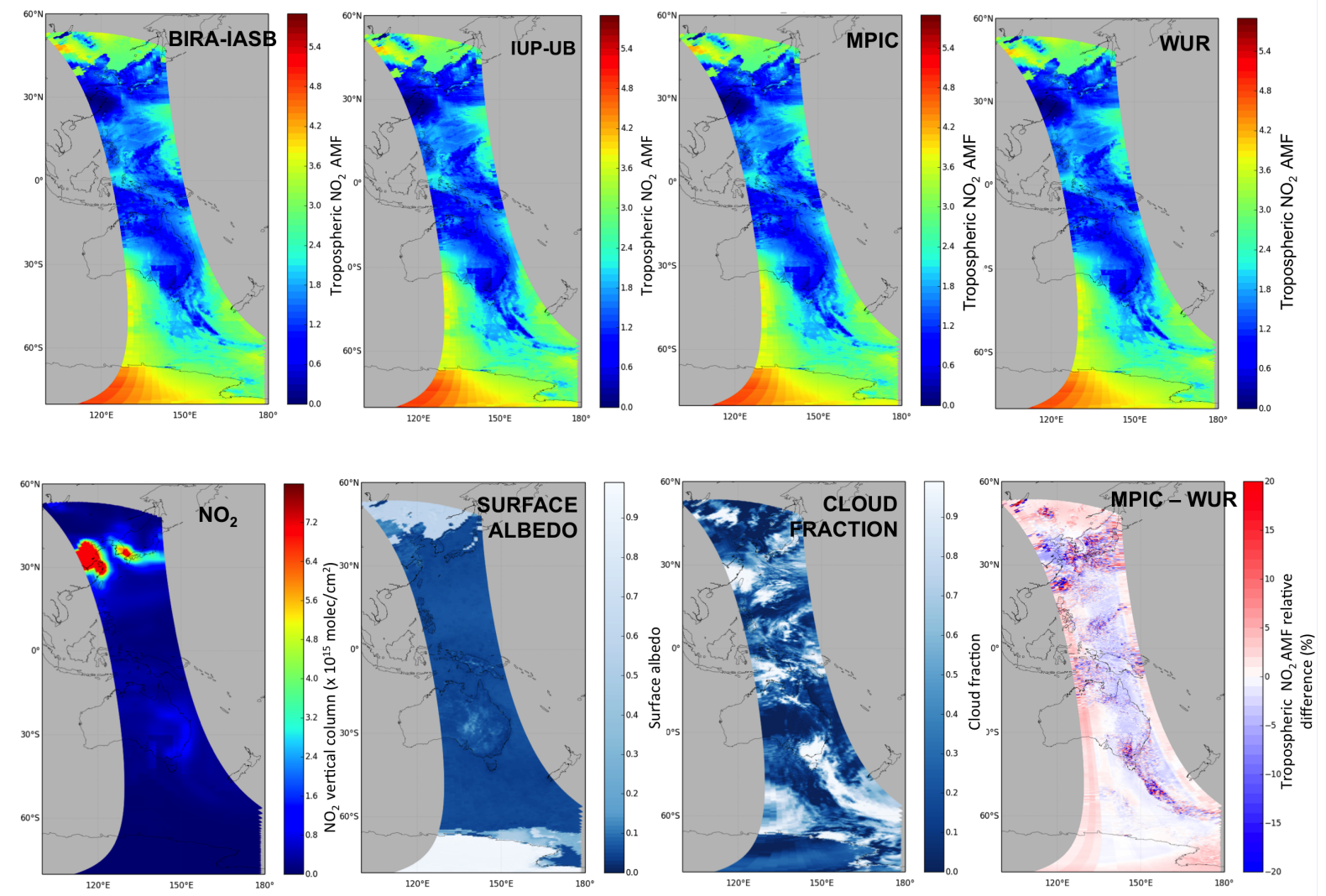

Figure 3.6: Upper panels: total $\mathrm{NO}_{2}$ tropospheric AMFs calculated by BIRA, IUP-UB, WUR and MPI-C. Lower panels: $\mathrm{NO}_{2}$ model tropospheric vertical column (from a priori TM4 profile), climatological surface albedo (from Kleipool et al., 2008), cloud fraction (from $\mathrm{O}_{2}-\mathrm{O}_{2}$ and FRESCO+) and an example of the relative differences between MPI-C and WUR AMFs. Only pixels for SZA $<70^{\circ}$ are shown. The selected OMI orbit is from 02 February 2005 (2005m0202-o02949-v003).

errors that are intrinsic to the calculation method using Eq. 3.3, i.e. interpolation errors in finding the AMF value from the 6-D LUT and the vertical discretization of the a priori profile. Overall, the average differences between the AMFs (always below 6.5\% for cloud fractions less than 0.2) are somewhat higher than the differences from the LUT comparison $(2 \%)$. This means that in successive steps of the AMF calculation sources of systematic uncertainty are added that propagate throughout the AMF calculation process. These sources directly affect the agreement between the AMF calculated by different groups and hence the AMF structural uncertainty. 6.5\% represents an upper limit value for the differences that using different RTMs and LUTs may introduce in the final AMF calculation. 

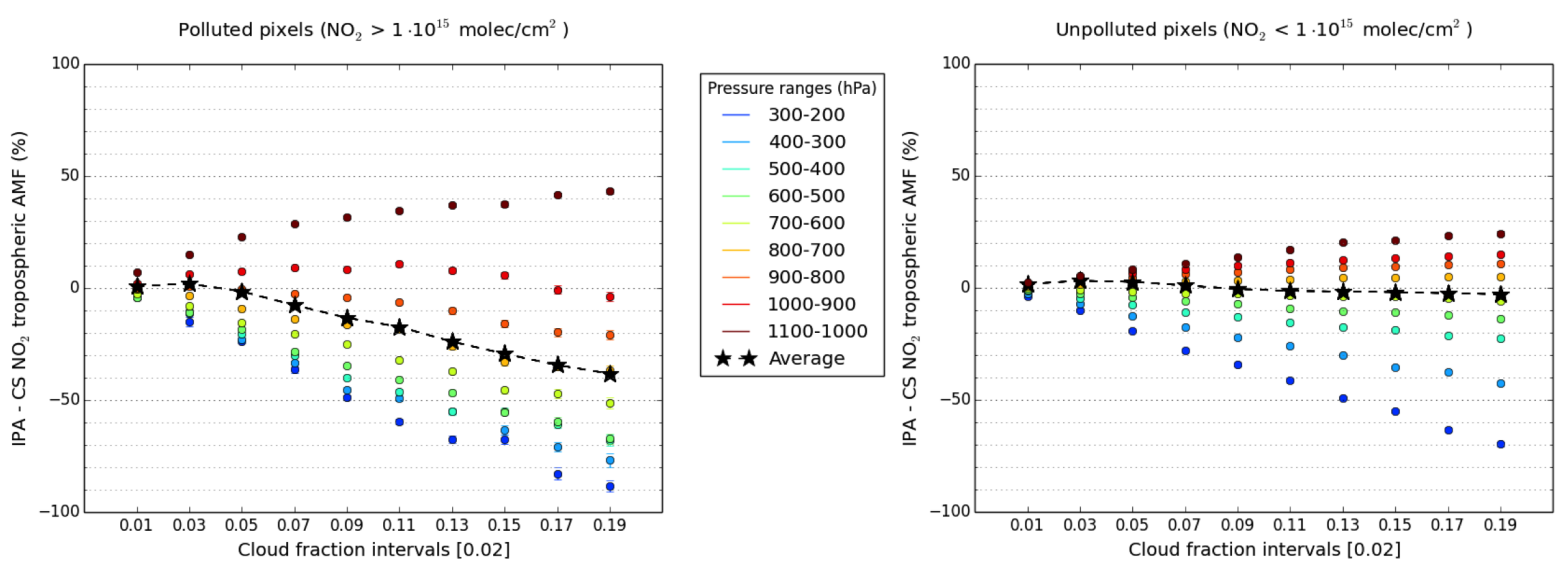

Figure 3.7: Mean relative differences between IPA and clear-sky $\mathrm{NO}_{2}$ tropospheric AMFs for different cloud fraction intervals at different cloud pressures ranges (different colors) for a complete day of OMI measurements (02 February 2005). Left panel is for polluted situations and right panel for unpolluted situations (pixels with model $\mathrm{NO}_{2}$ vertical column higher or lower than $1 \cdot 10^{15} \mathrm{molec} / \mathrm{cm}^{2}$ respectively). The stars with the black dashed lines show the average difference for all the cloud pressures. Pixels with surface albedo less than 0.3 and SZA $<70^{\circ}$ are considered.

\section{Cloud correction: IPA vs. clear-sky AMF}

It is important to account for the effect of clouds on the photon path lengths in the troposphere when calculating tropospheric AMFs. There are various approaches that are commonly used to calculate AMFs in (partly) cloudy situations. The independent pixel approximation (IPA), introduced in Eq. 3.4 (e.g. Martin et al. (2002)), is motivated by the fact that few pixels are completely cloud-free. Many pixels still have some degree of cloud cover, and even small cloud fractions strongly affect the sensitivity to the trace gas. The relevant physical effect of clouds (reduced sensitivity to trace gas below the cloud and enhanced sensitivity to trace gas above and in the top layer of the cloud) is explicitly taken into account in the IPA. Another approach is to consider clear-sky (CS) AMF for scenes with a sufficiently small cloud fraction (e.g. Richter and Burrows (2002)). The motivation for using clear-sky AMFs instead of IPA is that for scenes with small cloud fractions (e.g. $<0.2$ ), retrieved cloud parameters (cloud fraction and cloud pressure) have relatively high uncertainty. This inhibits the reliable modelling of the effect of clouds on photon path lengths, and consequently, a clear-sky AMF is used.

To quantify the differences between the two approaches, we compare here tropospheric $\mathrm{NO}_{2}$ AMFs calculated by WUR (see Table 3.3) with the IPA approach and the clear-sky AMFs, for two complete days of OMI measurements (02 February 2005 and 16 August 2005). In polluted situations, IPA AMFs are smaller than CS AMFs on average, with differences as large as $-40 \%$ for cloud fractions approaching the threshold value of 0.2 (left panel of Fig. 3.7). The negative differences between IPA and CS AMFs are largest 
for the highest clouds, illustrating the reduced sensitivity to tropospheric $\mathrm{NO}_{2}$ below the cloud in the IPA. IPA AMFs are larger than clear-sky AMFs for clouds situated in the lower troposphere (cloud pressure $>900 \mathrm{hPa}$ ), where most $\mathrm{NO}_{2}$ pollution resides. These positive differences can be understood from the albedo effect of residual clouds. Low, bright clouds lead to enhanced photon scattering through the $\mathrm{NO}_{2}$ layers above the cloud level and also inside the cloud top layer, and this increases the sensitivity to $\mathrm{NO}_{2}$. For polluted situations, IPA AMFs are on average smaller than CS AMFs by $20 \%$ for cloud fractions of $0.05-0.2$, and smaller by $11 \%$ for cloud fractions between 0.0-0.2.

In unpolluted situations, IPA and CS AMFs are generally quite similar, with average relative differences within 5\%. Still, there are important differences between the two approaches. In unpolluted situations with clouds in the free and upper troposphere (cloud pressure $<600 \mathrm{hPa}$ ), IPA AMFs are smaller because of reduced sensitivity to $\mathrm{NO}_{2}$ (right panel of Fig. 3.7). For clouds in the lower troposphere, IPA AMFs are larger because of the albedo effect. The change of sign in the differences between IPA and CS AMFs now occurs near $700 \mathrm{hPa}$ (instead of near $900 \mathrm{hPa}$ for polluted scenes), reflecting the more even vertical distribution of $\mathrm{NO}_{2}$ in pristine situations compared to polluted scenes when most $\mathrm{NO}_{2}$ resides in the polluted boundary layer.

These results indicate that the differences between using IPA or clear-sky AMFs are substantial especially for polluted situations and small residual cloud fractions. Selecting a particular cloud correction approach implies that AMF values will be systematically different from values obtained with the other method. In polluted situations, the mean differences are 20-40\% for cloud fractions between 0.1-0.2, with cloud pressure largely explaining the magnitude and sign of the differences. Note that the a priori profiles used to calculate the AMFs in this section have been obtained from a specific CTM. If a different CTM were used, the values for the differences between IPA and CS AMFs would be different, in line with the structural uncertainty that is being discussed in this study (See Sect. 3.3.3). A previous study by Van Noije et al. (2006) reported $30 \%$ higher GOME tropospheric $\mathrm{NO}_{2}$ columns retrieved using the IPA compared to retrievals using clear-sky AMFs. Such differences are in line with the systematically lower IPA AMFs found here. But, like the study by Van Noije et al. (2006), we cannot clearly recommend one AMF approach over the other. In order to make such a recommendation, a more detailed analysis of the cloud parameter uncertainties is needed, along with a validation of tropospheric $\mathrm{NO}_{2}$ retrievals using different AMF approaches against independent reference data. Such a validation exercise should preferably focus on polluted situations with small (0.05-0.2) residual cloud fractions. 


\section{Round robin comparison}

For the round robin comparison, each group calculated tropospheric $\mathrm{NO}_{2}$ AMFs using their preferred settings (i.e. their own preference for source of forward model parameters, cloud and aerosol correction). We extended the comparison and included other leading international retrieval groups (University of Leicester, NASA and Peking University). We now have a wider range of approaches and assumptions to better evaluate the impact that the calculation methods and choices of forward model parameters have on the structural uncertainty.

Table 3.3 summarizes the AMF algorithms included in this comparison. There are several differences with the harmonized settings used in the previous section. IUP-UB and BIRA now apply IPA only when cloud fraction exceeds 0.1 and 0.2 , respectively, motivated by the high uncertainty of cloud parameters for scenes with small cloud fractions (see Sect. 3.3.3). Peking University accounts for the surface reflectance anisotropy and they do pixel-by-pixel online radiative transfer calculations. They also include an explicit aerosol correction, motivated by the fact that the implicit aerosol correction breaks down in situations of high aerosol optical thickness and strongly absorbing particles (Castellanos et al. (2015), Chimot et al. (2016)), which is particularly significant in East China. MPIC applies IPA cloud correction for clouds higher than 3km and clear-sky AMFs for clouds between 2 and $3 \mathrm{~km}$ when cloud fraction is less than 0.1 . For clouds below $2 \mathrm{~km}$ they include a parametrized aerosol-cloud layer in order to account for the possibility of cloud aerosol mixtures, which might be especially relevant for AMF calculation in scenarios where trace gas is most abundant in the lowest part of the troposphere. Among all the groups, five different chemistry transport models for the a priori $\mathrm{NO}_{2}$ profiles are used.

Different groups use different LUTs for their AMF calculations, and POMINO uses pixel-by-pixel online radiative transfer calculations. The LUTs are different in several aspects: the RTMs used to create them and the number of reference points for each dimension. All these differences affect the AMF structural uncertainty. Based on the discussion in previous sections we consider that the use of different LUTs introduces a structural uncertainty of the order of $6.5 \%$.

Most of the surface albedo values used in the retrievals come from the Kleipool et al. (2008) database, which is based on OMI surface reflectance climatology. However, due to the different representations of surface reflectance within this database (mode and min LER), only three retrieval groups use the exact same albedo values. We investigated if this could bias the estimation of the AMF structural uncertainty. We re-calculated the AMF structural uncertainty with two retrievals that use the same exact albedo values and with three that use different albedo values. These estimated AMF structural uncertainties 
were of similar magnitude and therefore we can conclude that the fact that the surface albedo values come from the Kleipool et al. (2008) database is not a clear driver of the overall structural uncertainty calculation.

The agreement of AMFs from this round robin exercise quantifies the overall AMF structural uncertainty. The comparison with 7 groups allowed us to calculate a mean AMF as a reference (which is not necessarily the true AMF) value which can be considered a state-of-the-art AMF value. For a representative ensemble mean AMF, we required all groups to have a valid (un-flagged) AMF value at a pixel location. We selected two different days (02 February 2005 and 16 August 2005) in winter and summer to identify possible seasonality effects in the agreement of the AMFs.

\section{Round robin: identical cloud parameters}

First we compare the 6 groups that use the same cloud parameters. In contrast to what we found in the harmonized settings comparison, the global maps of tropospheric AMF calculated by each group using their preferred settings (Fig. 3.8) show pronounced differences in several regions. For example, over the Sahara desert, where surface albedo is high (see lower panel on Fig. 3.8), AMFs differ by up to 15\%. Small differences in the albedo values can lead to high differences in the AMFs, especially for surface albedo lower than 0.3 (see Fig. 3.4b). Over Central Africa, AMFs differ in situations where cloud fraction is close to the typically applied threshold of 0.2 (left lower panel in Fig. 3.8).

We compared global AMF calculations from all individual groups against the pixel mean AMF from 6 groups (Peking University only calculates AMFs over China). Figure 3.9 shows the average ratio of the AMF by each group to the ensemble mean AMF (bars) and the correlation (crosses) for polluted situations $\left(\mathrm{NO}_{2}>1 \cdot 10^{15} \mathrm{molec} / \mathrm{cm}^{2}\right.$, left panel) and unpolluted situations $\left(\mathrm{NO}_{2}<1 \cdot 10^{15} \mathrm{molec} / \mathrm{cm}^{2}\right.$, right panel). Over polluted regions (for pixels with SZA $<60^{\circ}$ and effective cloud fraction $<0.2$ ), the agreement among the 6 groups is within (minimum-maximum) 12-42\% in February and within 10-31\% in August. BIRA AMFs are 14\% higher than the ensemble mean, and WUR AMFs are $18 \%$ lower, suggesting considerable structural uncertainty.

Over unpolluted regions the agreement is better: AMFs from the different groups agree within 8.5-18\% in both February and August, which implies a smaller structural uncertainty (Table S3.6 provides a detailed summary of the comparison).

In order to asses which forward model parameters explain most of the AMF structural uncertainty, we analyzed AMF differences from groups that use identical cloud parameters and implicit aerosol correction (BIRA, University of Leicester, NASA and WUR). Between these four groups, the only different forward model parameters are surface albedo, 


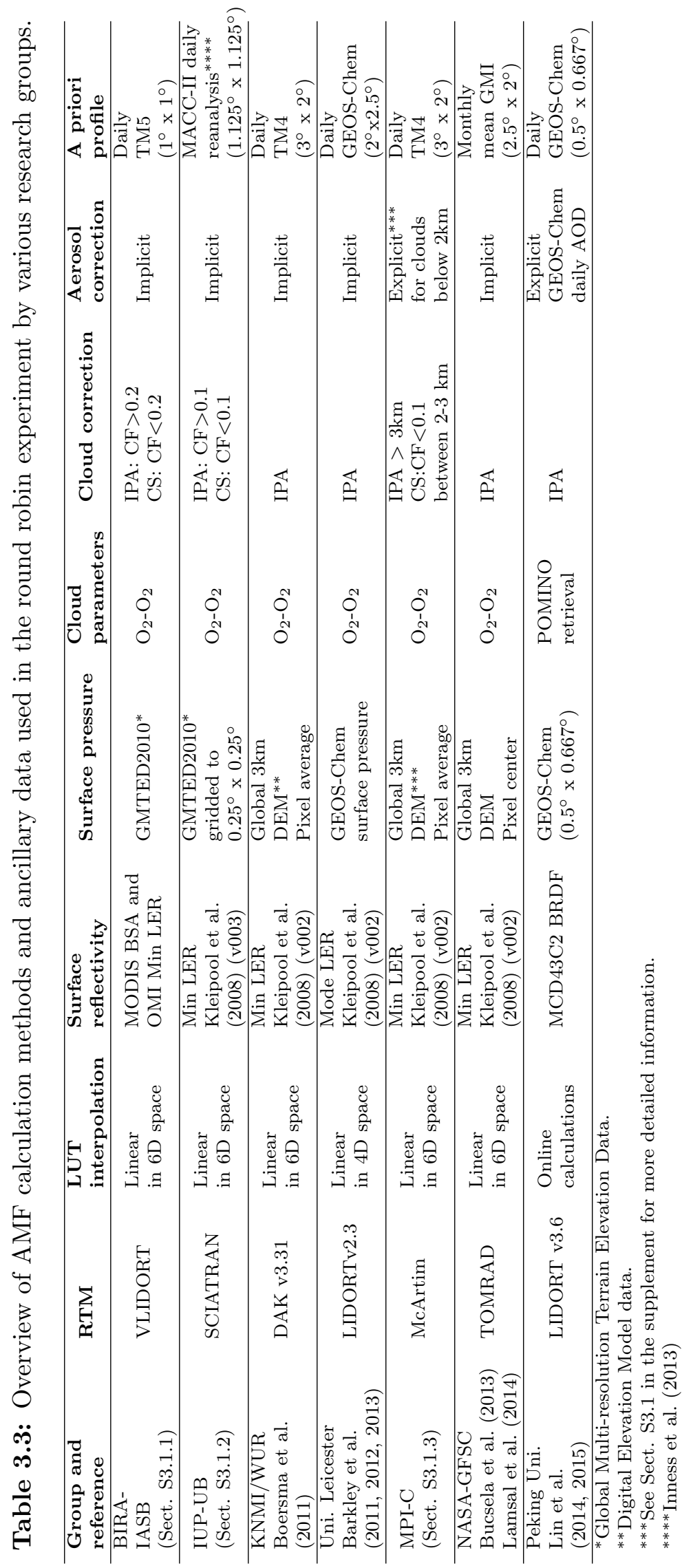



for $\mathrm{NO}_{2}$ and $\mathrm{HCHO}$ satellite retrievals
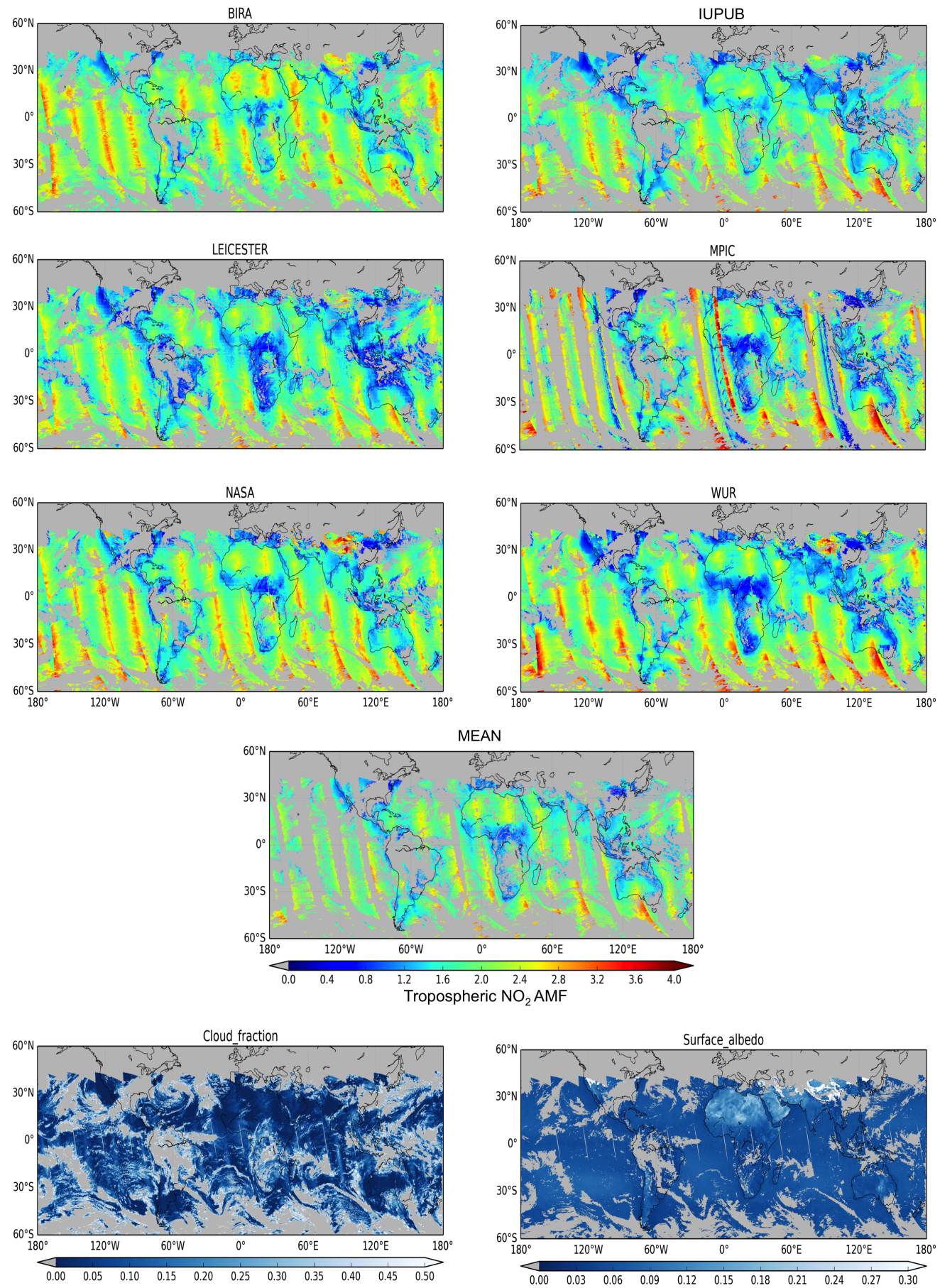

Figure 3.8: Tropospheric $\mathrm{NO}_{2}$ AMFs calculated by each of the groups for a complete day of OMI measurements (02 February 2005). Lower panels show an example of cloud fraction and surface albedo used by KNMI/WUR (showed as example; see Table 3.3) to calculate the AMFs. Groups apply different filters to the measurements which explains the different gaps (grey). 
a priori $\mathrm{NO}_{2}$ profile and surface pressure. To investigate which of these parameters best explains the AMF variability, we correlated differences between a particular parameter $\left(\Delta \mathrm{A}_{\mathrm{s}}, \Delta \mathrm{NO}_{2}\right.$ and $\left.\Delta \mathrm{P}_{s}\right)$ with the corresponding AMF differences $(\Delta \mathrm{AMF})$. For each particular parameter, we required the differences in the other parameters to be small (surface albedo within \pm 0.02 , surface pressure within $\pm 50 \mathrm{hPa}$ and a priori $\mathrm{NO}_{2}$ vertical columns within $\left.\pm 0.2 \cdot 10^{15} \mathrm{molec} / \mathrm{cm}^{2}\right)$ so we could isolate the effect of one parameter only, while keeping sufficient pixels for statistical significance.

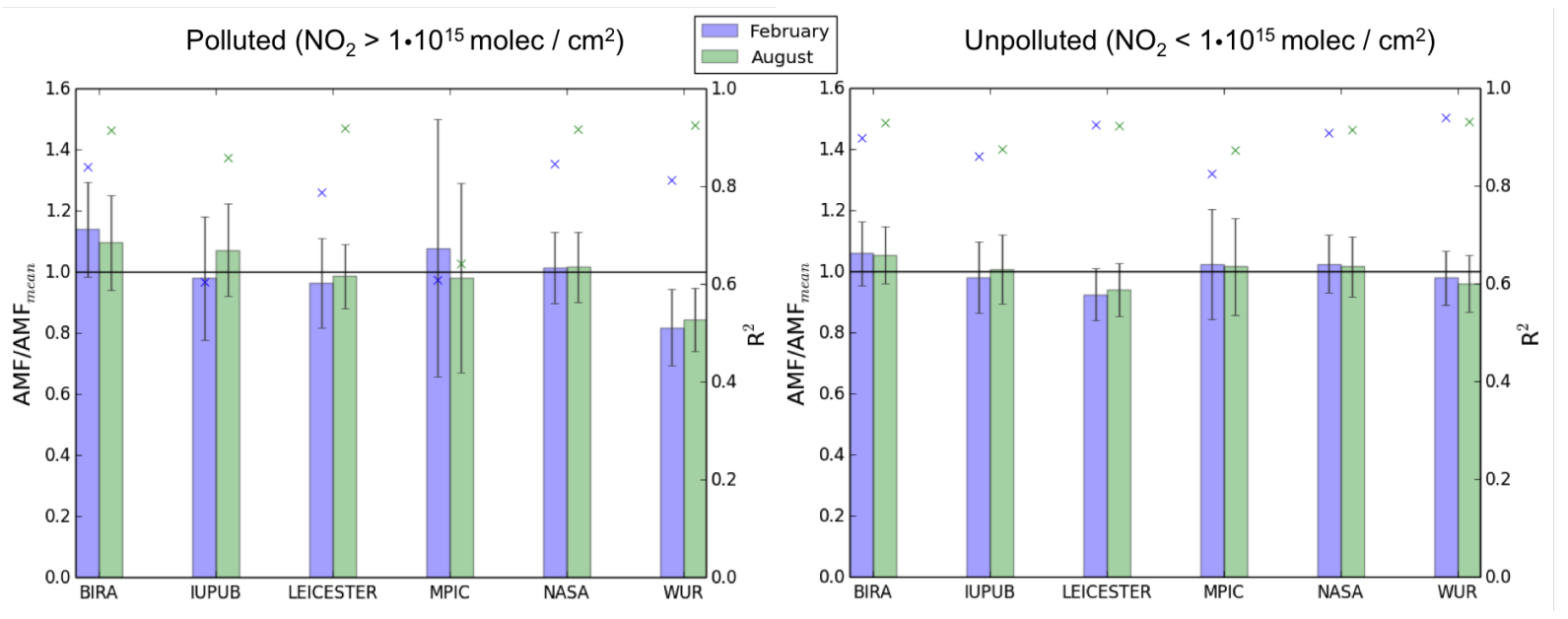

Figure 3.9: Ratio of tropospheric $\mathrm{NO}_{2}$ AMFs by each group to the ensemble mean (left axis, bars) and the correlation coefficient (right axis, cross) for two complete days of OMI measurements (02 February 2005 (blue) and 16 August 2005 (green)) over the globe for polluted (left panel) and unpolluted (right panel) pixels. The error bars correspond to the standard deviation. Only pixels for SZA $<60^{\circ}$ and cloud fraction $<0.2$ are considered in the analysis.

We focus on explaining the differences between BIRA and WUR here, since these were on the order of $30 \%$ (Fig. 3.9). We explored the correlations between BIRA-WUR AMF differences and differences between assumed surface pressures, albedos, and $\mathrm{NO}_{2}$ vertical columns and profile shapes; results are shown in Fig. S3.3 and Table S3.3. We find that surface pressure differences do not explain the large systematic AMF differences, and that surface albedo differences explain WUR and BIRA AMF differences especially in winter, when $\mathrm{NO}_{2}$ is found close to the surface and AMFs are more sensitive to albedo variations than in summer. In our ensemble, the WUR-BIRA AMF differences are highly sensitive to the differences between the a priori $\mathrm{NO}_{2}$ profiles used, especially in summer. $\mathrm{NO}_{2}$ profiles are vertically more elevated in TM5 (used by BIRA) than in TM4 (used by WUR) (right panel of Fig. S3.3), as diagnosed by their $20 \mathrm{hPa}$ lower effective $\mathrm{NO}_{2}$ pressures (pressure levels weighted by $\mathrm{NO}_{2}$ sub-column in that level). The confinement of the trace gas to lower atmospheric layers and the higher concentrations explains the systematically lower AMF values for WUR compared to BIRA.

Selecting a specific chemistry transport model thus influences the AMF structural 
uncertainty via differences in the profile shape. These differences in the profile shape depend on the different characteristics of the models (e.g. spatial and temporal resolution and parametrization of different processes in the atmosphere). Previous studies analysed how using different CTMs influences the $\mathrm{NO}_{2}$ retrievals due to the change in the profile shapes used to calculate the AMF values. Heckel et al. (2011) compared retrievals using fine and coarse resolution models and concluded that using one AMF value for a large heterogeneous scene can lead to $50 \%$ bias in the retrieved $\mathrm{NO}_{2}$ columns. Vinken et al. (2014) reported much smaller average differences of $10 \%$ in retrieved $\mathrm{NO}_{2}$ columns mainly due to different emission inventories used in TM4 $\left(3^{\circ} \times 2^{\circ}\right)$ and GEOS-Chem $\left(0.5^{\circ} \times 0.67^{\circ}\right)$. According to Laughner et al. (2016), different temporal resolution also influences a priori profile shapes; they found differences in the retrieved $\mathrm{NO}_{2}$ column for individual days up to $40 \%$ that were mostly explained by day-to-day wind direction variations that were not captured in the monthly averages.

All these aspects influence the estimation of retrieval (and AMF) theoretical uncertainties. In order to quantitatively estimate the effect of one model characteristic alone (e.g. the spatial resolution) on the AMF structural uncertainty it would be necessary to compare AMF calculated with the same approach but with just that specific characteristic being different in the profile shapes generated by the CTM. Such a specific sensitivity analysis has not been done in this study but should be considered in future AMF comparisons. To test the robustness of our structural uncertainty estimate, we did some experiments by simulating the effect of high resolution a priori profiles on AMF values. Kuhlmann et al. (2015), McLinden et al. (2014) and Heckel et al. (2011) reported that AMFs calculated using coarse resolution a priori profiles are overestimated over polluted areas by approximately 50\%. Over remote locations, there is little spatial variability in $\mathrm{NO}_{2}$ distributions, and the a priori profile spatial resolution is less important in the AMF calculation. When including synthetic AMF emulating the use of high resolution a priori profiles over polluted areas, the estimated AMF structural uncertainty is not strongly affected (increases by 3-6\%). This indicates that with the ensemble of retrievals used in our comparison the estimate of the structural uncertainty in the AMF calculation may be considered a robust estimate.

The findings in this subsection indicate that quality assurance efforts for retrievals should not focus just on column validation, but also target the validation of the a priori $\mathrm{NO}_{2}$ profiles used in the AMF calculations. It is worth to note that using averaging kernels in satellite applications (e.g. when comparing retrieved $\mathrm{NO}_{2}$ columns with modelled $\mathrm{NO}_{2}$ distributions or observed $\mathrm{NO}_{2}$ profiles) will reduce the representativeness errors in the comparisons associated with the a priori trace gas profile used in the retrieval scheme (e.g. Boersma et al. (2016b)) . 


\section{Round robin: different cloud parameters}

In the previous section, we found that differences between a priori $\mathrm{NO}_{2}$ profiles and surface albedo values are the main cause for AMF structural uncertainty when cloud parameters are identical in AMF calculation approaches. Here we extend our round robin experiment by including AMF calculations from Peking University (Lin et al. (2014, 2015)) that were done with different cloud parameters (Table 3.3) than the $\mathrm{O}_{2}-\mathrm{O}_{2}$ cloud parameters used by all other groups. The comparison of Peking University and WUR AMFs thus allowed us to investigate the relative importance of differences in cloud parameters in driving AMF structural uncertainty. Our comparison of AMFs is confined to China, since Peking University calculations are only available over that region.

All the groups calculate similar spatial patterns for the AMFs over China (Fig. 3.10). In the polluted northeast (Beijing area) the AMFs are lower due to the reduced sensitivity to $\mathrm{NO}_{2}$ in the lower troposphere. In the western part over the Tibet region, AMFs are higher due to the presence of ice and snow in February. Figure 3.11 shows the average ratio of each group's AMF to the ensemble mean AMF (bars) and the correlation (crosses) for polluted situations (left panel) and unpolluted situations (right panel). In polluted regions, AMFs generally agree within 37\% in February and within 20\% in August, and correlations are 0.7-0.9. Peking University AMFs are higher than the ensemble mean AMF, especially in August when they are 25\% higher. WUR and MPI-C AMFs are lower than the mean AMF, especially in August (20\% lower). In unpolluted regions the agreement is better: within $26 \%$ in February and within $16 \%$ in August, with correlation of 0.8-0.95 (see Table S3.7).

To estimate the effect of differences in cloud parameters on AMF structural uncertainty, we analysed differences in AMF calculated by WUR and Peking University. The Peking University AMF calculations (and the cloud parameters) were based on a version of the POMINO retrieval using clouds retrieved with an implicit aerosol treatment (i.e. similar to KNMI/WUR). We explored the correlations between Peking University and WUR AMFs differences and differences in cloud pressure $\left(\mathrm{P}_{c}\right)$ and $\mathrm{NO}_{2}$ vertical columns by requiring the differences in other forward model parameters to be relatively small. Results are shown in Fig. S3.4 and Table S3.4. AMF differences are partly explained by differences in the effective cloud pressures (Table S3.4): the $\mathrm{O}_{2}-\mathrm{O}_{2}$ cloud pressures used by WUR are systematically lower (by $100 \mathrm{hPa}$ ) than those by Peking University, in line with (Veefkind et al., 2016). This results in stronger screening of below-cloud $\mathrm{NO}_{2}$ pollution, and consequently lower AMFs by WUR compared to Peking University AMFs. Peking University uses $\mathrm{NO}_{2}$ profiles from GEOS-Chem. These profiles tend to peak at higher vertical levels than those from TM4 (Lin et al. (2014), Boersma et al. (2016b)), thus contributing to higher AMFs by Peking University compared to WUR AMFs. In summary, the more elevated $\mathrm{NO}_{2}$ profiles in combination with less elevated clouds explain 

for $\mathrm{NO}_{2}$ and $\mathrm{HCHO}$ satellite retrievals
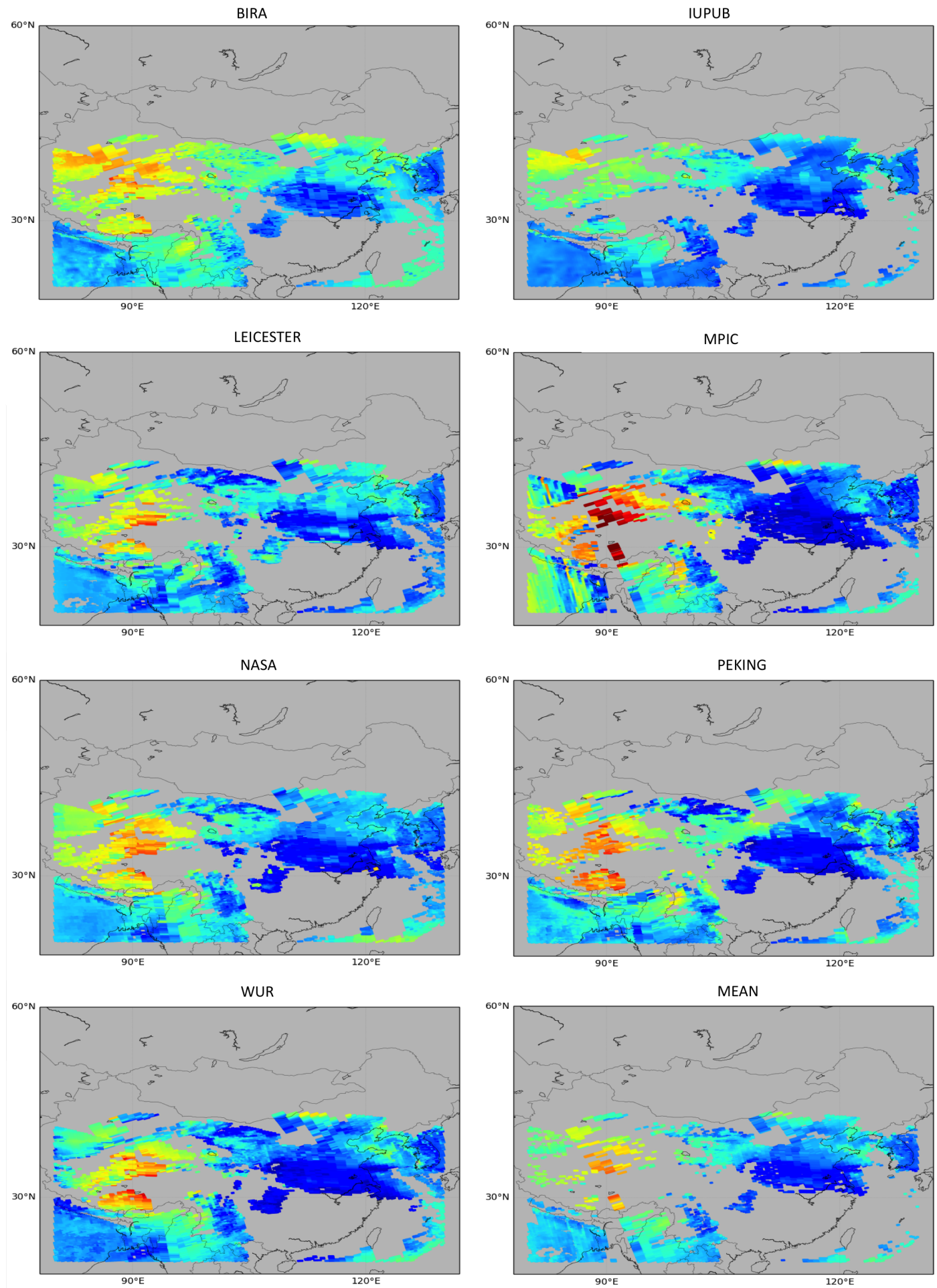

Figure 3.10: Tropospheric $\mathrm{NO}_{2}$ AMFs calculated by each of the groups for a complete day of OMI measurements (02 February 2005) over China $\left(20^{\circ} \mathrm{N}-53^{\circ} \mathrm{N} / 80^{\circ} \mathrm{W}-130^{\circ} \mathrm{W}\right)$. Only pixels for SZA $<60^{\circ}$, effective cloud fraction $<0.5$ and surface albedo $<0.3$ are shown. 


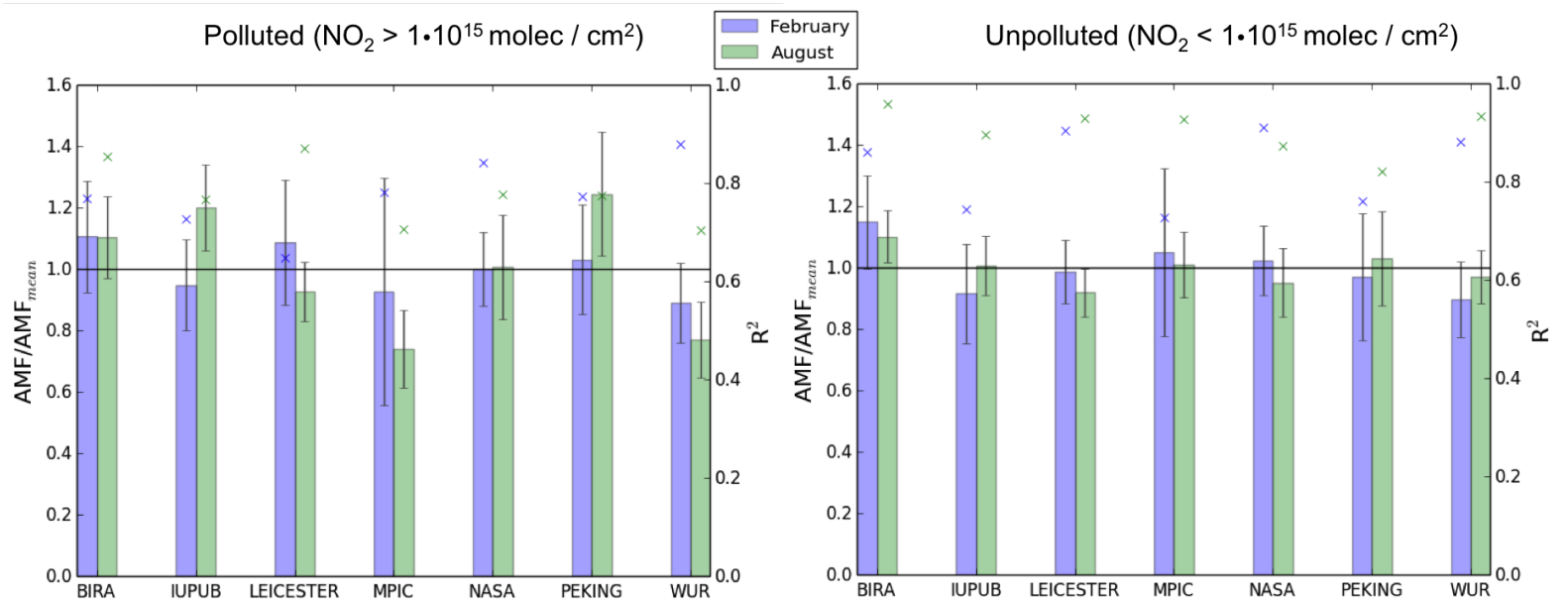

Figure 3.11: Ratio of tropospheric $\mathrm{NO}_{2}$ AMFs by each group to the ensemble mean (left axis, bars) and the correlation coefficient (right axis, cross) for two complete days of OMI measurements (02 February 2005 (blue) and 16 August 2005 (green)) for polluted (left panel) and unpolluted (right panel) pixels over China. The error bars correspond to the standard deviation. Only pixels for SZA $<60^{\circ}$ and cloud fraction $<0.2$ are considered in the analysis.

the substantially higher AMF by Peking University than WUR AMFs.

\section{Round robin: explicit aerosol correction}

The POMINO retrieval by Peking University explicitly corrects for the presence of aerosols in the atmosphere by including profiles of aerosol optical properties simulated by the GEOS-Chem model (and constrained by MODIS AOD on a monthly basis) in the radiative transfer model and in the cloud retrieval (Lin et al. (2014, 2015)). All the other groups except MPIC-C (see Table 3.3 and Sect. S3.1.3) assume that the aerosol effects are implicitly accounted for in the cloud retrievals (Boersma et al. (2011), Castellanos et al. (2015)). Including an explicit aerosol correction influences AMF values indirectly by changes in cloud fraction and cloud pressure and directly in the radiative transfer simulations. We quantify the effect of the choice of aerosol correction in AMF structural uncertainty by comparing AMFs calculated by Peking University with (abbreviated $\mathrm{AMF}_{\text {aer }}$ hereafter) and without (AMF) explicit aerosol correction.

In situations with substantial aerosol pollution (AOD > 0.5), selection of one aerosol correction approach over the other can result in AMF structural uncertainty of $45 \%$ over China. The sign of the AMF differences depends mainly on the altitude of the aerosol layer relative to the $\mathrm{NO}_{2}$ profile (see e.g. Leitao et al. (2010)). We find that $\mathrm{AMF}_{\text {aer }}$ are on average $55 \%$ smaller in situations when aerosols are located above the $\mathrm{NO}_{2}$ layer, mainly because cloud pressures are lower on average (more than $350 \mathrm{hPa}$ ), resulting in stronger screening of $\mathrm{NO}_{2}$ (upper panel of Fig. S3.5; Table S3.5). When the aerosol 
vertical distribution is similar to that of $\mathrm{NO}_{2}, \mathrm{AMF}_{\text {aer }}$ are on average $45 \%$ higher, mostly because of much smaller cloud fractions, resulting in reduced screening of below-cloud $\mathrm{NO}_{2}$ (lower panel in Fig. S3.5; Table S3.5). An additional factor is that when aerosols are mixed with $\mathrm{NO}_{2}$, they increase the optical light path and enhance AMF values. These results are in line with Lin et al. (2015) where an evaluation of the influence of the aerosols in the $\mathrm{NO}_{2}$ retrieval is analyzed for 2012 .

\subsubsection{Stratospheric air mass factors}

We pointed out in Sect. 3.3.3 that differences in the description of the atmosphere's sphericity could lead to differences in stratospheric AMFs, especially for extreme geometries. Here we investigate the differences between stratospheric $\mathrm{NO}_{2}$ AMFs calculated with DAK and McArtim radiative transfer models. The McArtim model simulates the radiative transfer in an atmosphere that is spherical for incoming, single-scattered, and multiple-scattered light. DAK's atmosphere is spherical for incoming sunlight, but planeparallel for scattered sunlight. Based on these differences, we may expect the average photon paths at high altitudes in McArtim to be shorter than in DAK, as diffuse photon contributions (from near-horizontal directions) in McArtim are bound to finite spherical atmosphere (as illustrated in Figure S3.2). Consequently, stratospheric AMFs in McArtim are smaller (Fig. 3.4a). Figure 3.12 shows that McArtim box-AMFs (at $25 \mathrm{hPa}$ ) are systematically lower than those from DAK by $1-2 \%$ for moderate viewing geometries, with more significant differences (up to $-5 \%$ to $-10 \%$ ) when solar zenith and viewing angles are large.
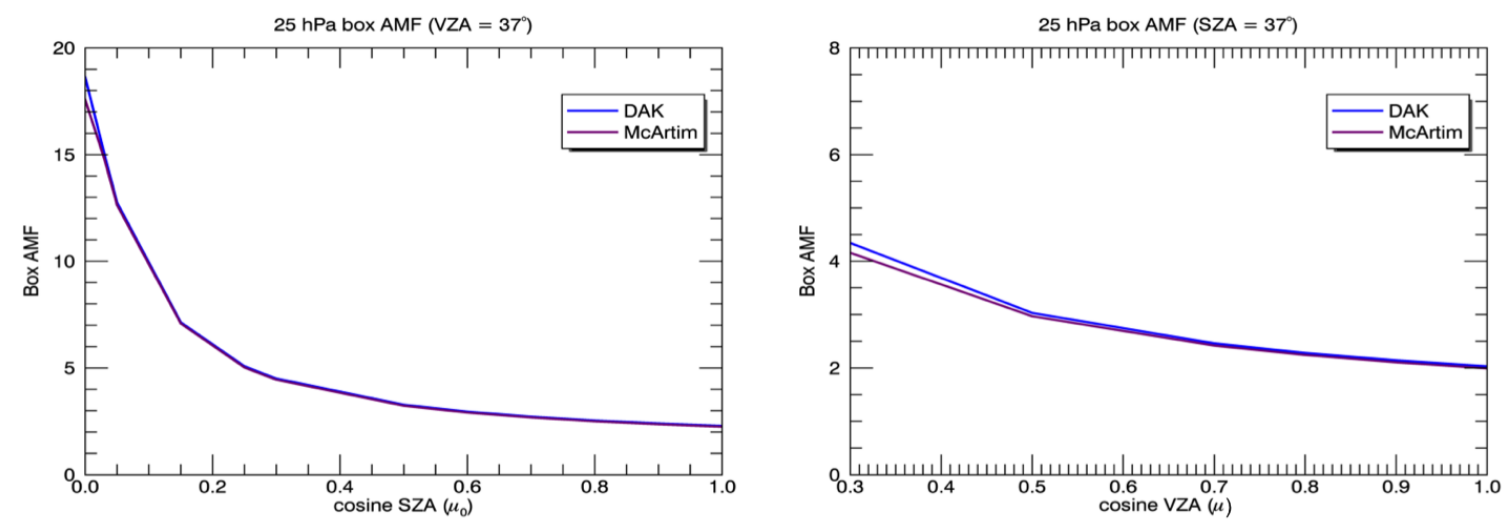

Figure 3.12: Box-AMFs at $25 \mathrm{hPa}$ as a function of cosine of $\mathrm{SZA}$ (left panel) and as a function of cosine of VZA (right panel). In the left panel, VZA is constant at $37^{\circ}(\mu=0.8)$, and at the right panel, SZA is constant at $37^{\circ}\left(\mu_{0}=0.8\right)$.

A direct validation of stratospheric $\mathrm{NO}_{2}$ AMFs is difficult, but comparing simulated stratospheric slant column densities against observed $\mathrm{NO}_{2}$ SCDs constitutes a test of 
the radiative transfer models. Here we use OMI-observed (un-destriped) SCDs over the Pacific from the OMNO2A v1 product (van Geffen et al. (2015), Boersma et al. (2011)) as benchmark. The $\mathrm{NO}_{2}$ columns over the Pacific Ocean are dominated by stratospheric $\mathrm{NO}_{2}$, so we expect simulated stratospheric SCD values to be similar or somewhat smaller than the observed, total SCDs. Simulated SCDs are the product of modelled VCDs (from data assimilation in TM4) and the stratospheric AMFs calculated with DAK and McArtim. Figure 3.13 (left panel) indicates (for high solar and viewing zenith angles) that stratospheric SCDs simulated with McArtim are close to, or slightly below the OMI SCDs. In contrast, the stratospheric SCDs simulated with DAK overtop the OMI SCDs, because of the higher stratospheric AMFs from that model. This inevitably leads to negative values for SCD-SCD $\mathrm{Strat}_{\text {, }}$, and consequently to reduced or even negative tropospheric $\mathrm{NO}_{2}$ VCDs at high latitudes. Indeed, DOMINO v2 retrievals (using DAK stratospheric AMFs) are known to suffer from negative tropospheric VCDs at high latitudes especially in the summer hemisphere (Beirle et al., 2016) when solar zenith angles are largest. For small solar zenith angles in the Tropics, the differences between DAK and McArtim stratospheric slant columns are smaller, but still appreciable at the edges of the swath (Fig. 3.13 (right panel)).
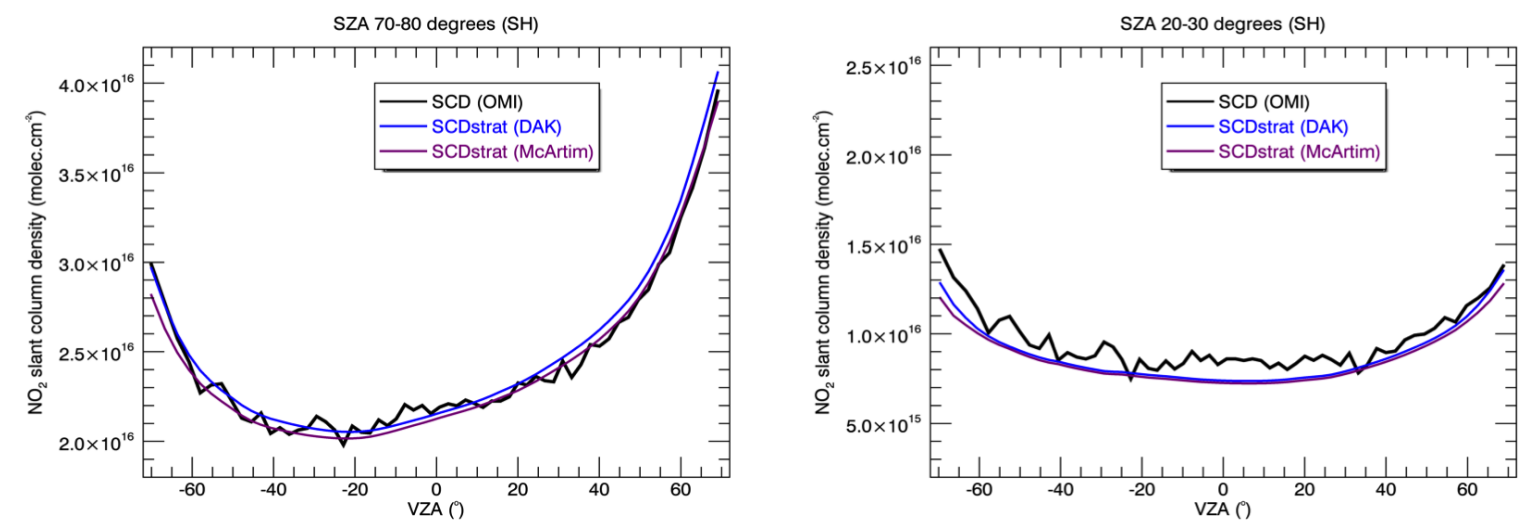

Figure 3.13: Averaged OMI total $\mathrm{NO}_{2} \mathrm{SCD}$ (black line) as a function of viewing zenith angle for solar zenith angles between 70-80 degrees (left panel) and 20-30 degrees (right panel) (OMI orbit 02940 on 02 February 2005). The blue line indicates the estimated stratospheric SCDs based on DOMINO v2 stratospheric VCDs and DAK stratospheric AMFs, and the purple line represents the stratospheric SCDs based on DOMINO v2 stratospheric VCDs and McArtim stratospheric AMFs. The only difference between the DAK and McArtim-based stratospheric slant columns is the use of the radiative transfer model; all other relevant parameters (TM4 assimilated stratospheric column, cloud parameters, albedo, $\mathrm{NO}_{2}$ profile shape) are identical.

We tested whether possible errors in the diurnal cycle of stratospheric $\mathrm{NO}_{2}$ could explain the overestimated slant columns for extreme viewing geometries. We did so by imposing stratospheric $\mathrm{NO}_{2}$ vertical columns that are either constant with OMI row number (i.e. with local time), or increase (as $\mathrm{N}_{2} \mathrm{O}_{5}$ photolysis, $\mathrm{NO}_{2}$ concentrations build up) 
at a rate of approximately $0.1510^{15} \mathrm{molec} / \mathrm{cm}^{2} \mathrm{~h}^{-1}$, i.e. by $110^{15} \mathrm{molec} / \mathrm{cm}^{2}$ from the left to the right side of the orbit (Fig. S3.6a). These estimates correspond to the range of increase rates at high latitudes in summer reported in the literature (e.g. Vaughan et al. (2006), Celarier et al. (2008), Dirksen et al. (2011)). Our tests show that for these scenarios, simulated SCDs based on McArtim generally stay within the observational constraints of the OMI SCD patterns but that the simulated SCDs based on DAK are still exceeding the observed SCDs (Fig. S3.6b-c). McArtim provides a better physical description of photon transport in the stratosphere. The results above are not yet fully conclusive; a complete test would require the implementation of McArtim (instead of DAK) in the data assimilation scheme, or a dedicated validation of $\mathrm{NO}_{2}$ columns with independent reference data in situations with extreme viewing geometries. Nevertheless, our results clearly hint at McArtim as the RTM providing the more realistic stratospheric AMFs, and we will test this assumption further in the remainder of the QA4ECV project.

\subsection{Conclusions}

We have analysed in detail the AMF calculation process for $\mathrm{NO}_{2}$ and $\mathrm{HCHO}$ satellite retrievals from seven different retrieval groups. By comparing approaches for every step of the AMF calculation process we have identified the main sources of structural uncertainty and we have traced back these uncertainties to their underlying causes. We have estimated the structural uncertainty in the $\mathrm{NO}_{2}$ AMF calculation, which results from methodological choices and from preferences and assumptions made in the calculation process. Structural uncertainty is relevant beyond theoretical algorithm uncertainty, which typically only addresses the propagation of errors within the context of one particular retrieval algorithm.

The choice of RTM for TOA reflectance and box-AMF calculation introduces an average uncertainty of 2-3\%. The detailed comparison showed that state-of-the-art RTMs are in good agreement. Particularly for DAK, this is the first time that box-AMF calculations are extensively tested against those calculated with other RTMs. The McArtim model simulates systematically lower box-AMFs in the stratosphere, which we attribute to the model's geometrically more realistic description of photon scattering in a spherical atmosphere. The four European retrieval groups agree within $6 \%$ in their calculation of $\mathrm{NO}_{2}$ tropospheric AMFs when identical ancillary data (surface albedo, terrain height, cloud parameters and a priori trace gas profile) and cloud correction are used. This demonstrates that the selection of RTM and the interpolation operations lead to modest uncertainty, which is intrinsic to the calculation method chosen and therefore cannot be avoided. 
When retrieval groups use their preference for ancillary data along with their preferred cloud and aerosol correction, we find that the structural uncertainty of the AMF calculation is $42 \%$ over polluted regions and $31 \%$ over unpolluted regions. Table 3.4 shows the escalation of the structural uncertainty with every step of the AMF calculation. The steep increase from $6 \%$ to $42 \%$ strongly suggests that it is not the models or the calculation method but the assumptions and choices made to represent the state of the atmosphere that introduce most structural uncertainty in the AMF calculation. The structural uncertainty is of similar magnitude as the theoretical uncertainties found in algorithm error propagation studies which confirms that there is a substantial systematic component in trace gas satellite retrieval uncertainties.

Table 3.4: Average relative structural uncertainty for every step of the AMF calculation following the comparison process shown in Fig. 3.1. This includes the modelling of TOA reflectance $\left(\sigma_{R}\right)$, calculation of box-AMF LUT $\left(\sigma_{m}\right)$, tropospheric AMFs using harmonized settings $\left(\sigma_{M}\right)$ and the overall structural uncertainty from AMF using preferred settings $\left(\sigma_{M^{\prime}}\right)$.

\begin{tabular}{ccccc}
\hline & $\sigma_{R}$ & $\sigma_{m}$ & $\sigma_{M}$ & $\sigma_{M^{\prime}}$ \\
\hline $\mathbf{N O}_{2}$ & $1.1 \%$ & $2.6 \%$ & $6 \%$ & $31 \%-42 \%$ \\
\hline $\mathbf{H C H O}$ & $1.5 \%$ & $2.6 \%$ & & \\
\hline
\end{tabular}

Sensitivity studies for one particular algorithm indicate that the choice for cloud correction (IPA or clear-sky AMF for small cloud fractions) is a strong source of structural uncertainty especially for polluted situations with residual cloud fractions of 0.05-0.2 (on average an structural uncertainty of $20 \%$ ). The choice for aerosol correction (explicitly or implicitly via the cloud correction) introduces an average uncertainty of $50 \%$, especially when aerosol loading is substantial. Selecting trace gas a priori profiles from different chemistry transport models, surface albedo from different datasets and cloud parameters from different cloud retrievals contributes substantially to structural uncertainty in the AMFs. These findings point to the need for detailed validation experiments designed to specifically test cloud and aerosol correction methods under relevant conditions (strong pollution, residual cloud fractions of 0.1-0.2). Not just the retrieved $\mathrm{NO}_{2}$ column itself should be validated, but also the a priori vertical $\mathrm{NO}_{2}$ profile, the cloud and aerosol distributions, and the surface albedo values should be compared in detail against independent reference measurements.

The magnitude of the structural uncertainty in AMF calculations is significant, and is caused mainly by methodological differences and particular preferences for ancillary data between different retrieval groups. This study provides evidence for the need of improvement of the different ancillary datasets, including uncertainties of the forward model parameters used in the retrievals for a better agreement in the AMF calculation. This will decrease significantly AMF structural uncertainty towards the levels desired in user requirement studies $( \pm 10 \%)$. As there is no "true" AMF value to be used as 
reference, it is difficult to decide which approach and which ancillary data are the best. For this reason, future research should include a thorough validation against independent reference data, specifically in the situations where AMF structural uncertainty has highest impact.

\section{Acknowledgements}

This research has been supported by the FP7 Project Quality Assurance for Essential Climate Variables (QA4ECV), grant No. 607405. A.H. and A.R. acknowledge funding by DLR in the scope of the Sentinel-5 Precursor verification project (grant 50EE1247). UoL acknowledges the use of the ALICE and SPECTRE High Performance Computing Facility at the University of Leicester. We would like to thank the two anonymous referees for the useful interactive discussion in the review process. 


\section{The importance of surface re- flectance anisotropy for cloud and $\mathrm{NO}_{2}$ retrievals from GOME-2 and OMI}

This chapter is published as:

Lorente, A., Boersma, K. F., Stammes, P., Tilstra, L. G., Richter, A., Yu, H., Kharbouche, S., and Muller, J. P.: The importance of surface reflectance anisotropy for cloud and $\mathrm{NO}_{2}$ retrievals from GOME-2 and OMI, Atmos. Meas. Tech., 11, 4509-4529, doi: 10.5194/amt-11-4509-2018, 2018 


\section{Abstract}

The angular distribution of the light reflected by the Earth's surface influences top-ofatmosphere (TOA) reflectance values. This surface reflectance anisotropy has implications for $U V /$ Vis satellite retrievals of albedo, clouds, and trace gases such as nitrogen dioxide $\left(\mathrm{NO}_{2}\right)$. These retrievals routinely assume the surface to reflect light isotropically. Here we show that cloud fractions retrieved from GOME-2A and OMI with the FRESCO and OMCLDO2 algorithms have an East-West bias of $10 \%$ to $50 \%$, highest over vegetation and forested areas, and that this bias originates from the assumption of isotropic surface reflection. To interpret the across-track bias with the DAK radiative transfer model, we implement the Bidirectional Reflectance Distribution Function (BRDF) from the Ross-Li semi-empirical model. Testing our implementation against state-of-art RTMs LIDORT and SCIATRAN, we find that simulated TOA reflectance generally agrees to within $1 \%$. We replace the assumption of isotropic surface reflection in the equations used to retrieve cloud fractions over forested scenes with scattering kernels and corresponding BRDF parameters from a daily, high-resolution database derived from 16 years' worth of MODIS measurements. Doing so, the East-West bias in the simulated cloud fractions largely vanishes. We conclude that across-track biases in cloud fractions can be explained by cloud algorithms not adequately accounting for the effects of surface reflectance anisotropy. The implications for $\mathrm{NO}_{2}$ air mass factor (AMF) calculations are substantial. Under moderately polluted $\mathrm{NO}_{2}$ and backscatter conditions, clear-sky AMFs are up to $20 \%$ higher and cloud radiance fractions up to $40 \%$ lower if surface anisotropic reflection is accounted for. The combined effect of these changes is that $\mathrm{NO}_{2}$ total AMFs increase by up to $30 \%$ for backscatter geometries (and decrease by up to 35\% for forward scattering geometries), stronger than the effect of either contribution alone. In an unpolluted troposphere, surface BRDF effects on cloud fraction counteract (and largely cancel) the effect on the clear-sky AMF. Our results emphasize that surface reflectance anisotropy needs to be taken into account in a coherent manner for more realistic and accurate retrievals of clouds and $\mathrm{NO}_{2}$ from $\mathrm{UV} /$ Vis satellite sensors. These improvements will be beneficial for current sensors, in particular for the recently launched TROPOMI instrument with a high spatial resolution. 


\subsection{Introduction}

Nitrogen dioxide $\left(\mathrm{NO}_{2}\right)$ in the lower troposphere is an important constituent of air pollution. In Europe, the annual mean $\mathrm{NO}_{2}$ concentration limit value $\left(40 \mu \mathrm{g} / \mathrm{m}^{3}\right)$ is still widely exceeded, exposing 30 million people to poor air quality with known harmful health effects (EEA, 2016). In combination with other pollutants and sunlight, chemical and physical transformations of nitrogen oxides $\left(\mathrm{NO}+\mathrm{NO}_{2}=\mathrm{NO}_{\mathrm{x}}\right)$ lead to the formation of particulate matter and ozone smog, further impacting public health, ecosystems, and climate. Satellite measurements of tropospheric $\mathrm{NO}_{2}$ column densities provide much better spatial coverage than ground-based sensors, and they have been used to monitor trends, and to estimate NOx emissions and $\mathrm{NO}_{2}$ surface concentrations (e.g. Richter et al. (2005), Martin et al. (2003), Lamsal et al. (2008)). The spatial resolution of the satellite instruments and their retrievals is improving such that the observed $\mathrm{NO}_{2}$ pollution can now be traced back to emissions from individual cities, power plants, and transportation sectors. The uncertainty of satellite $\mathrm{NO}_{2}$ retrievals is considerable, and mainly related to the adequacy of the assumptions made on the state of the atmosphere. We recently estimated the structural uncertainty from an ensemble of $\mathrm{NO}_{2}$ retrievals to be on the order of 30$40 \%$ (Lorente et al., 2017). An important component of this uncertainty is how surface properties (usually from an external database) are taken into account, and how errors in the external database propagate in the air mass factor (AMF) calculations. This is not straightforward, because the AMF calculation directly depends on surface properties under clear-sky circumstances, and indirectly via cloud parameters retrieved for the same scene by the cloud algorithm.

Surfaces reflect light differently in each direction, and the angular distribution of the reflected light influences top-of-atmosphere (TOA) reflectance levels measured by satellite instruments that monitor atmospheric composition. Therefore, surface reflectance anisotropy influences retrievals of surface albedo, trace gases, aerosols and clouds from satellite instruments like the Global Ozone Monitoring Experiment 2 (GOME-2) and the Ozone Monitoring Instrument (OMI). In surface albedo, cloud, aerosols and trace gas retrievals, the surface is often assumed to be Lambertian: an idealized surface that reflects light isotropically (e.g. Kleipool et al. (2008), Veefkind et al. (2016), Torres et al. (2007), Boersma et al. (2011)). This assumption implies that the geometry dependent scattering properties of the reflecting surface are ignored.

The so-called Lambertian-equivalent-reflectivity (LER) climatologies represent the albedo of the Lambertian surface in the radiative transfer simulations for cloud retrievals and trace gas retrievals. In constructing such climatologies (e.g. monthly climatologies), a large ensemble of measurements taken over a scene over multiple years is analyzed statistically, and based on the lower $1 \%$ percentile reflectance, an inversion is done to retrieve 
the surface reflectance (e.g. Koelemeijer et al. (2003), Kleipool et al. (2008), Tilstra et al. (2017)). Depending on the exact viewing and illumination geometry however, the surface may appear darker or brighter. Taking the lower $1 \%$ percentile reflectances therefore skews the distribution of retrieved albedo values to those scenes that appear darker from space. Using these climatologies therefore fails to represent any surface reflectance anisotropic effects on TOA reflectance simulations for the widely varying subset of viewing geometries encountered along a satellite orbit. We may expect cloud retrievals to be directly affected by the assumption of a Lambertian surface in the radiative transfer: if a scene is brighter than predicted by the biased climatology, cloud fractions will be overestimated. Trace gas retrievals are affected by the Lambertian assumption in the calculation of the AMF: directly via the clear-sky AMF and also indirectly because the retrieved cloud parameters are used to correct for the possible presence of residual clouds via the independent pixel approximation (IPA) method. In the IPA, the cloud radiance fraction weighs the clear-sky and cloudy parts of the scene for the calculation of the overall AMF and vertical column density (VCD) (e.g. Martin et al. (2002)).

The angular distribution of the reflected light by a surface is represented mathematically by the Bidirectional Reflectance Distribution Function (BRDF) (Nicodemus et al., 1992). Anisotropy is a fundamental physical property of surface reflectance, so in order to fully represent the geometry dependent surface scattering properties in cloud and trace gas retrievals, surface BRDF has to replace the isotropic Lambertian albedo. Some studies have already shown that surface BRDF effects are important for $\mathrm{NO}_{2}$ and cloud retrievals. Zhou et al. (2010) found that after including surface BRDF over Europe, differences in $\mathrm{NO}_{2}$ columns were higher in November (20\%) compared to July (3\%), when the solar zenith angle is small. Noguchi et al. (2014) studied surface BRDF effects on the diurnal cycle of clear-sky geostationary measurements over Japan, and found that whether $\mathrm{NO}_{2}$ columns are under $(-15 \%)$ or overestimated $(+9 \%)$ depends on the specific geometry of the measurement. To also address the need of including surface BRDF effects on cloud algorithms, Lin et al. (2014, 2015) updated the POMINO retrieval over China. Changes in surface reflectance led to changes of opposite sign in cloud fraction $( \pm 0.05)$ and more complex effects on cloud pressure, with an overall change of $10 \%$ in $\mathrm{NO}_{2}$ columns that were regionally and seasonally dependent. Vasilkov et al. (2017) created a geometry dependent surface LER (GLER) product and applied it to $\mathrm{OMI} \mathrm{NO}_{2}$ and cloud retrievals. They found relatively small effects on retrieved cloud parameters and relatively high differences in retrieved $\mathrm{NO}_{2}$ columns (up to 50\%) driven by GLER values being on average smaller than the original LER.

These studies show that surface BRDF effects depend on the specific geometry (hence local time and season) and surface characteristics of the individual measurements, and that averaging over many pixels results in smaller differences. They analysed mostly 
clear-sky scenes (i.e. no clouds present) or scenes with very low cloud fractions (i.e. lower than 0.2), and they did not consider how surface reflectance anisotropy affects the radiative transfer in the atmosphere and TOA reflectance. The indirect effect of biased cloud parameters on $\mathrm{NO}_{2}$ retrievals combined with effects on clear-sky AMFs received less attention. Vasilkov et al. (2017) and Lin et al. (2015) addressed the indirect effects and showed that the effects on cloud parameters could enhance or compensate the direct effect on clear-sky AMFs.

Here we study the effect of surface reflectance anisotropy on surface LER climatologies, on cloud fraction retrievals and on $\mathrm{NO}_{2}$ tropospheric AMFs, covering the essential steps from the TOA reflectance to retrieve the final $\mathrm{NO}_{2}$ tropospheric column in a partly cloud covered pixel. We present observational evidence that surface reflectance anisotropy skews LER climatologies to the darkest scenes corresponding with forward scattering geometries (Sect. 4.2). We demonstrate that using these LER climatologies in cloud retrieval algorithms leads to considerable across-track biases in cloud fractions, especially for the $\mathrm{O}_{2}$-A band (GOME-2), but also for the $477 \mathrm{~nm} \mathrm{O} \mathrm{O}_{2}-\mathrm{O}_{2}$ band (OMI). In Section 4.3 we describe our extension of the DAK radiative transfer model (RTM) to include surface reflectance anisotropy with the Ross-Li BRDF semi-empirical model. We validate DAK with stateof-the-art radiative transfer models and evaluate TOA reflectance simulations including surface BRDF at the relevant wavelengths for cloud and $\mathrm{NO}_{2}$ retrievals. In Sect. 4.4 we study the consequences of including surface BRDF in the calculation of effective cloud fractions. In Sect. 4.5 we study the surface BRDF effects on $\mathrm{NO}_{2}$ AMFs, and how these, in combination with the effects on cloud fractions, affect tropospheric $\mathrm{NO}_{2}$ retrievals in cloudy scenes. We end with conclusions and outlook.

\subsection{Evidence of the influence of surface reflectance anisotropy on LER climatologies and cloud re- trievals}

Surface LER climatologies are commonly used as boundary conditions for cloud and trace gas retrievals (e.g. De Smedt et al. (2017), Bucsela et al. (2013)). Earlier instruments like GOME and SCIAMACHY have very coarse pixels $\left(40 \mathrm{x} 320 \mathrm{~km}^{2}\right.$ and $60 \mathrm{x} 30 \mathrm{~km}^{2}$, respectively) and a narrow swath $(960 \mathrm{~km})$. For retrievals from these instruments, the Lambertian assumption can be justified as surface BRDF effects are likely to smooth out over the large and heterogeneous pixels. Newer instruments like OMI, and especially TROPOMI, have a higher spatial resolution (13 x $24 \mathrm{~km}^{2}$ and $3.5 \times 7 \mathrm{~km}^{2}$, respectively) and a wider swath (up to $2600 \mathrm{~km}$ ), i.e. a wider range of viewing directions, and therefore the surface BRDF effects become more relevant. One of the advantages of using LER 
climatologies is that they have been derived from measurements of the satellite instrument itself. For example, Koelemeijer et al. (2003) climatology for GOME and SCIAMACHY, Kleipool et al. (2008) for OMI and Tilstra et al. (2017) for GOME-2 and SCIAMACHY. In constructing these climatologies, it is assumed that the surface reflectance is fully isotropic, and the angular dependence of reflected light is neglected. In the minimum-LER method, surface LER values are the $1 \%$ cumulative values retrieved from the histogram of Earth reflectance over a specific scene in a climatological period. The presence of clouds increases TOA reflectance compared to clear-sky scenes, therefore taking the minimum LER assures that the surface LER values represent mostly cloud-free scenes. However, if for particular viewing and illumination geometries, the TOA reflectance appears brighter, then those measurements will not be included in the climatology. This will introduce an intrinsic (but not explicit) viewing angle dependency in the surface LER climatologies derived from the satellites. That measurements taken under different viewing geometry configurations are used to create the climatologies, does not mean that climatological surface LER values are representative for typical or average viewing geometries of the instruments.

Figure 4.1a, b show the minimum surface LER climatology (2007-2013) at $494 \mathrm{~nm}$ and $772 \mathrm{~nm}$ for March over Amazonia for GOME-2 onboard MetOp-A (hereafter GOME2A) from Tilstra et al. (2017). This climatology was derived from the $1 \%$ cumulative reflectances gathered irrespective of viewing geometry. Figure 4.1c, d show the directional dependence of the minimum surface LER climatologies derived using the same GOME-2A measurements, but now discriminating between different geometries. For this purpose, the 24 measurements in 24 different viewing directions along the GOME-2 swath are considered independently, and then the minimum LER method is applied for each viewing direction as in the derivation of the original full swath climatology. We consider East as the 8 most eastward measurements, Nadir as the centered ones and West as the 8 most westward measurements. The average relative azimuth angle (RAA) characterizes the light scattering regime for measurements in each region: low RAA in the East (RAA $\left.=16^{\circ}\right)$ corresponds to forward scattering and high $\mathrm{RAA}\left(\mathrm{RAA}=164^{\circ}\right)$ in the West corresponds to backward scattering.

In Figs. 4.1c, d the horizontal line is the original surface LER value obtained using all GOME-2A measurements over Amazonia, and corresponds to the average value over the box in Fig. 4.1a, b $\left(\overline{A_{\mathrm{LER}}}=0.028\right.$ at $494 \mathrm{~nm}$ and $\overline{A_{\mathrm{LER}}}=0.21$ at $\left.758 \mathrm{~nm}\right)$. The albedo is lower at $494 \mathrm{~nm}$ because of absorption of light by chlorophyll. At $758 \mathrm{~nm}$, if only measurements of the eastern part (E) of the orbit are used to construct the climatology, the average surface LER value is slightly lower $\left(\overline{A_{\text {LER }}}=0.19\right)$ than the value using all measurements. If only nadir $(\mathrm{N})$ measurements are used, the average surface LER increases $\left(\overline{A_{\mathrm{LER}}}=0.24\right)$, and for the westernmost measurements $(\mathrm{W})$, the average surface 


\subsection{Evidence of the influence of surface reflectance anisotropy on LER climatologies and cloud retrievals}

LER increases to almost twice the original value $\left(\overline{A_{\mathrm{LER}}}=0.37\right)$. Using the full swath climatology thus implies a slight overestimation of the surface reflectance for the eastern measurements and a strong underestimation for nadir and western measurements. This systematic effect is a consequence of the directional signature of the surface reflectance. In the backward scattering direction, canopy surfaces generally appear brighter than in the forward scatter direction (e.g. Camacho-de Coca et al. (2004)). This effect is strongest in the near-infrared (NIR, $0.7-2.5 \mu \mathrm{m}$ ) spectral range where the atmosphere is more transparent, and over vegetation, which has non-isotropic elements (e.g. dense trees with heterogeneous leave orientation and shadowing effects). The effect in the surface LER climatologies also appears over non-vegetated regions and at shorter wavelengths. However, it is not as strong because stronger Rayleigh scattering tends to smooth out the sensitivity to surface effects and because land surfaces are darker at shorter wavelengths. Non-vegetated areas are usually more isotropic than vegetated areas. Because these biased surface LER climatologies are used in cloud retrievals (e.g. FRESCO Wang et al. (2008), $\mathrm{O}_{2}-\mathrm{O}_{2}$ Veefkind et al. (2016)), we anticipate a substantial effect on the retrieved cloud properties and as a consequence on trace gas column retrievals that use cloud parameters retrieved in the NIR, where sensitivity to surface anisotropy is strong (such as GOME-2).

Indeed, we find that cloud fractions retrieved with FRESCO cloud retrieval from GOME-2 measurements are affected by the across-track bias in the surface LER climatology. FRESCO retrieves cloud properties in the $\mathrm{O}_{2}$ A-band near $760 \mathrm{~nm}$. Figure $4.2 \mathrm{a}$ shows that over Amazonia (in March 2008) FRESCO cloud fractions are generally lower for the eastern measurements than for nadir and western measurements. This dependency can be explained by the directional biases in the surface LER (Fig. 4.1d). In the nadir and west measurements, the surface LER is underestimated and the retrieval compensates this overestimating cloud fractions in order to match observed TOA reflectances.

This results in higher mean effective cloud fractions for the nadir $\left(\overline{c_{\mathrm{eff}}}=0.50\right)$ and west $\left(\overline{c_{\text {eff }}}=0.66\right)$ measurements compared to the east measurements $\left(\overline{c_{\text {eff }}}=0.33\right)$. The East - West bias $\left(100 \cdot\left(\overline{c_{\text {eff,W }}}-\overline{c_{\text {eff, } \mathrm{E}}}\right) / \overline{c_{\text {eff, }}}\right)$ in the cloud fraction depends on the time of the year and the location. It is not only present over forested areas (i.e. Amazonia (50\%), Equatorial Africa (42\%) in March 2008) but also occurs over other regions (e.g. over Europe (25\%) and Asia (10\%), not shown). Furthermore, in the ensemble of western measurements in most of the regions there are very few cloud fraction values lower than 0.2 . This directly impacts trace gas retrievals, because a cloud fraction of 0.2 is often used as a threshold above which it is considered difficult to retrieve tropospheric $\mathrm{NO}_{2}$ columns (cloud screening effect). This bias in FRESCO cloud fractions is significantly higher than the cloud fraction retrieval uncertainty estimates of 0.05 due to surface albedo uncertainty (Koelemeijer et al., 2002), which underlines the need to correct for surface BRDF effects 


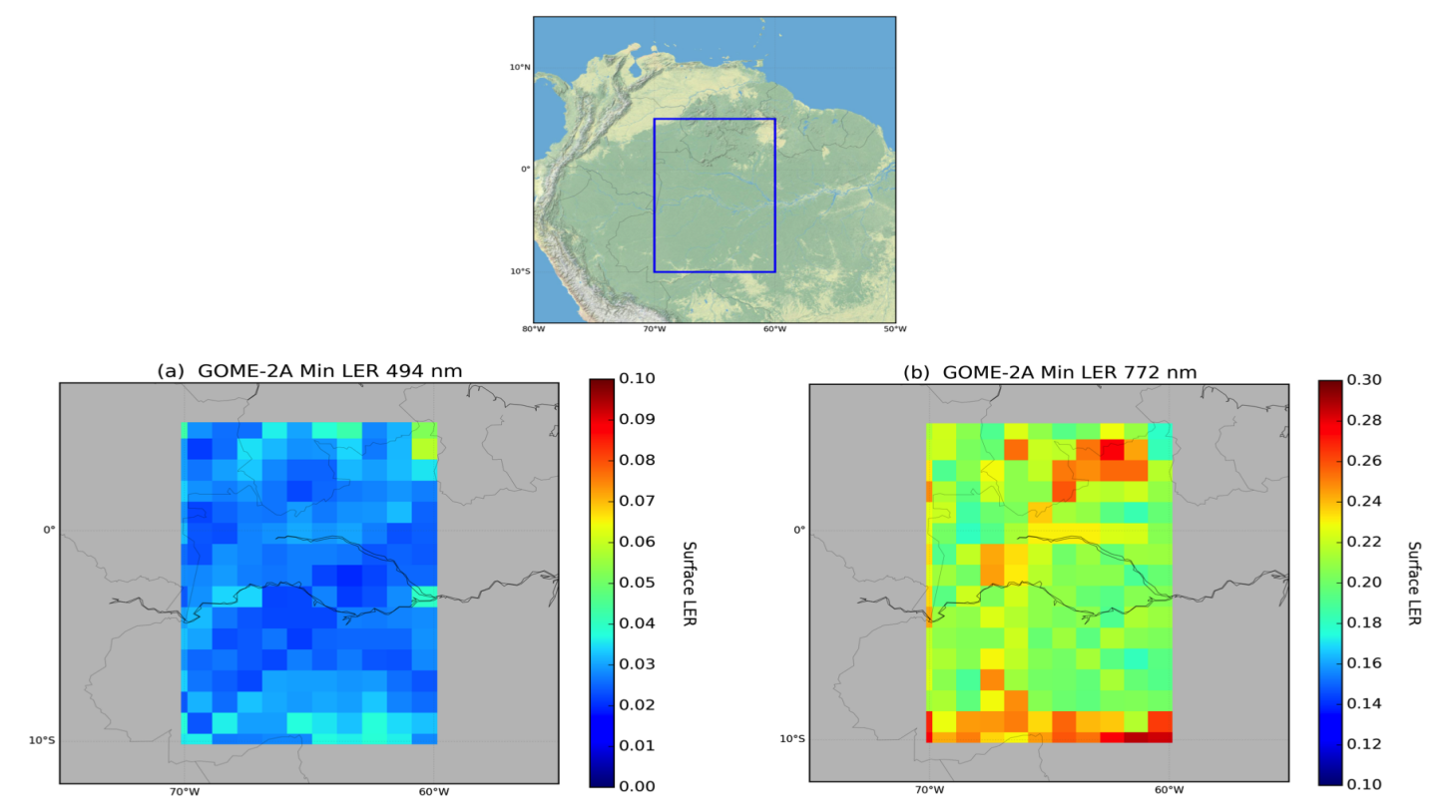

(c) Directional GOME-2A Min LER $494 \mathrm{~nm}$

(d) Directional GOME-2A Min LER $772 \mathrm{~nm}$
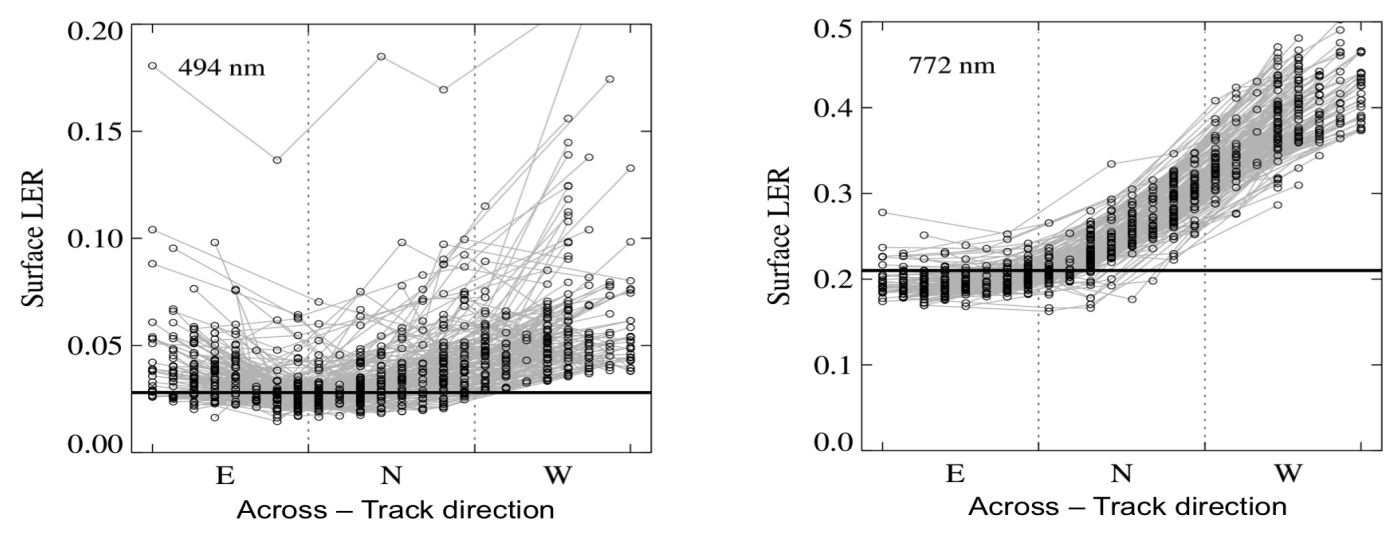

Figure 4.1: (a, b) Map of GOME-2A minimum surface LER climatology (2007-2013) for March at (a) $494 \mathrm{~nm}$ and (b) at $772 \mathrm{~nm}$ over Amazonia (Lat: 5N-10S, Lon: 60-70W, upper panel) at $1^{\circ} \times 1^{\circ}$ resolution. (c, d) Directional dependence of surface LER climatology (20072013) derived from individual measurements along the swath: East (E) for the 8 easternmost pixels, Nadir (N) for the 8 center pixels and West (W) for the 8 westernmost pixels. The horizontal line represents the surface LER using the full swath which is the average of the surface LER in panels $(\mathrm{a}, \mathrm{b})$.

in cloud retrievals in the $\mathrm{O}_{2}-\mathrm{A}$ band.

The OMCLDO2 cloud retrieval from the OMI retrieves cloud properties in the $\mathrm{O}_{2}-\mathrm{O}_{2}$ band around $470 \mathrm{~nm}$ and uses the Kleipool et al. (2008) surface LER climatology, which is based on the same principles as the climatology used in FRESCO. Cloud fractions from the OMI instrument retrieved with the OMCLDO2 algorithm also show a West - East ${ }^{1}$

\footnotetext{
${ }^{1} \mathrm{OMI}$ swath is divided into 60 different viewing directions; East corresponds to the 20 easternmost
} 

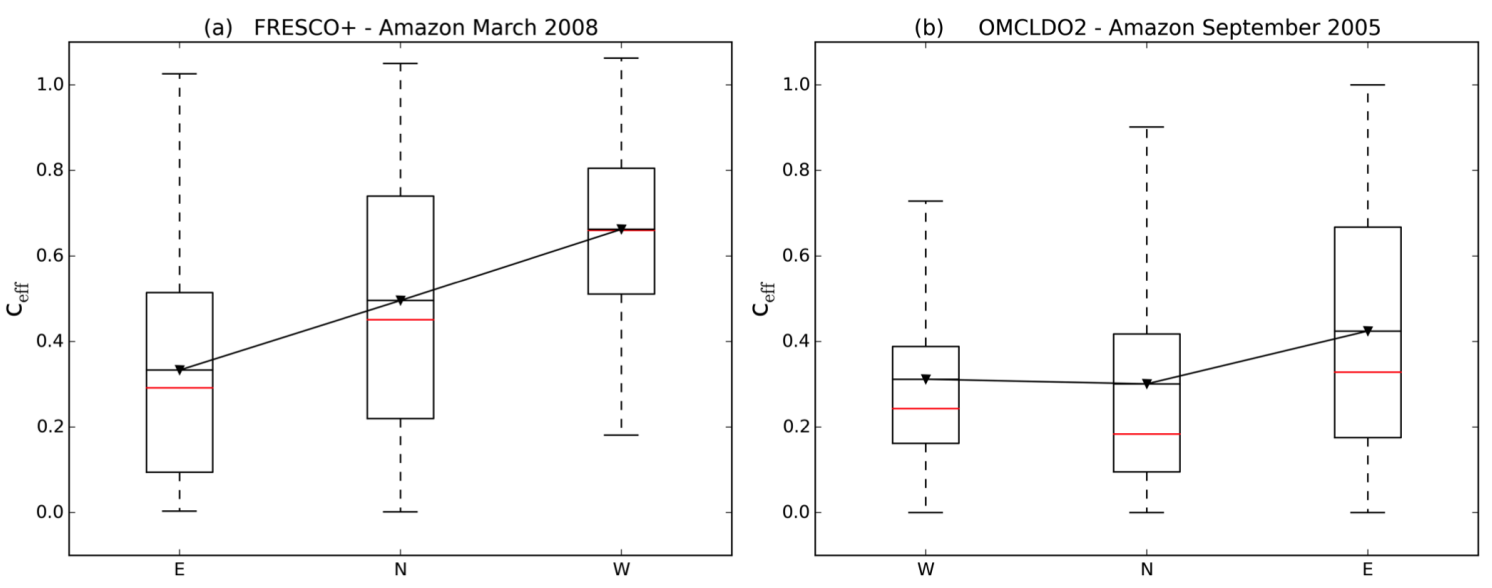

Figure 4.2: Box-plot of cloud fractions retrieved with (a) FRESCO cloud retrieval for GOME2A for March 2008 and (b) OMCLDO2 cloud retrieval for OMI for September 2005 for EastNadir- West measurements over Amazonia (Lat:5N-10S, Lon:60-70W). Black triangles correspond to mean values, red lines to median. The box represents 25 th and 75 th percentiles and the dashed lines the minimum and maximum values.

bias (Fig. 4.2b) over Amazonia (September 2005) of $26 \%\left(\overline{c_{\text {eff }, \mathrm{W}}}=0.31, \overline{\mathrm{c}_{\mathrm{eff}, \mathrm{E}}}=0.42\right.$ ) and around $15 \%$ over other regions. The effect is weaker than for GOME-2A (50\% vs. 26\% over Amazonia), but still substantial. The bias shown here is slightly higher than the cloud fraction retrieval uncertainty estimate which is always below 0.1 (Acarreta et al., 2004), suggesting that the bias in the $\mathrm{O}_{2}-\mathrm{O}_{2}$ retrieved cloud fractions can be significant depending on location and time of the year. Because OMI angles are larger than for GOME-2, a larger effect could be expected if also the $\mathrm{O}_{2}$-A band would have been used to retrieve clouds from OMI measurements.

We have shown that surface BRDF effects results in a distinct across-track bias in surface LER climatologies and cloud fractions retrieved from satellite instruments and that the effect is highly relevant in the NIR and in the visible. Errors in cloud fraction and surface albedo are the most important source of tropospheric AMF errors (Boersma et al., 2004), so we expect a strong impact on tropospheric $\mathrm{NO}_{2}$ retrievals. In the following section we describe how to account for surface anisotropy in the radiative transfer model DAK. Then, we study how cloud fraction and $\mathrm{NO}_{2}$ AMFs in the framework of cloud and trace gas retrievals are affected by the assumption of a Lambertian surface as compared to a realistic anisotropically reflecting surface. 


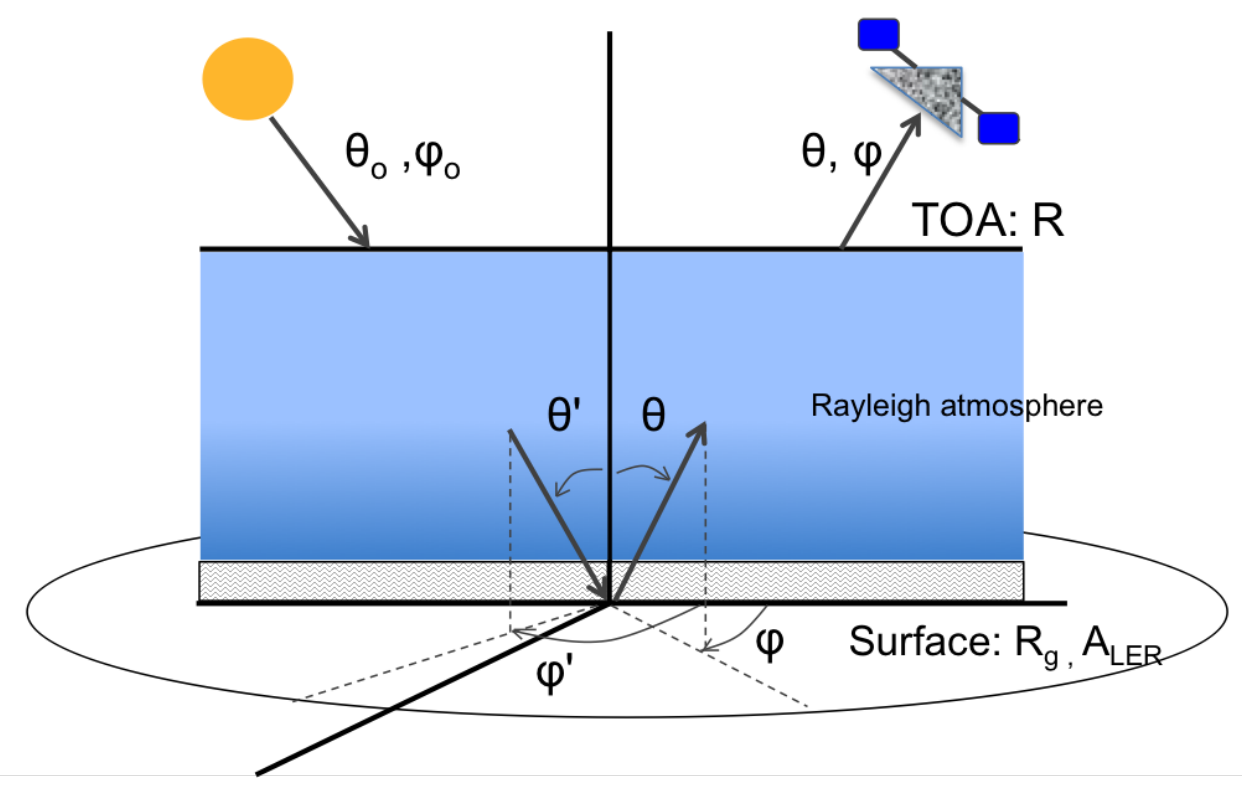

Figure 4.3: Sketch of the surface to top-of-atmosphere system with zenith and azimuth angles that define incident $\left(\theta^{\prime}, \varphi^{\prime}\right)$ and reflected $(\theta, \varphi)$ directions of light. Direct incident solar light is described by $\left(\theta_{0}, \varphi_{0}\right)$. $A_{\mathrm{LER}}$ is the Lambertian surface albedo and $R_{\mathrm{g}}$ the surface BRDF.

\subsection{Reflectance simulations with surface BRDF in DAK}

\subsubsection{Definition of BRDF}

The amount of radiation reflected by a surface in a certain direction depends on the direction of the incident irradiance and on the direction in which the reflected radiance is observed. The surface Bidirectional Reflectance Distribution Function (BRDF) is a function that characterizes the directional reflecting properties of a surface. The surface BRDF mathematically describes the angular distribution of the surface reflectance: $R_{\mathrm{g}}$ as a function of the illumination direction (incident, $\theta^{\prime}, \varphi^{\prime}$ ) and viewing direction (reflected, $\theta, \varphi)$ (see Fig. 4.3). It is expressed as the ratio of the reflected radiance in a certain direction $(d L)$ and the incident irradiance from a particular direction $\left(\mathrm{d} E^{\prime}\right)$ (Nicodemus et al., 1992):

$$
R_{\mathrm{g}}\left(\theta^{\prime}, \varphi^{\prime} ; \theta, \varphi\right)=\mathrm{d} L\left(\theta^{\prime}, \varphi^{\prime} ; \theta, \varphi\right) / \mathrm{d} E^{\prime}\left(\theta^{\prime}, \varphi^{\prime}\right) .
$$

The zenith angle $(\theta)$ and the azimuth angle $(\varphi)$ define the direction of incidence $\left(\theta^{\prime}, \varphi^{\prime}\right)$ and reflectance $(\theta, \varphi)$, as sketched in Fig. 4.3.

The albedo of a surface is generally defined as the ratio of the irradiance reflected by a surface area into the whole hemisphere and the irradiance incident on the surface with 
hemispherical angular extent (i.e. coming from all directions) (Schaepman-Strub et al., 2006). For particular illumination and viewing conditions, black-sky and white-sky albedo are defined, and they are obtained through hemispherical integration of the surface BRDF. The black-sky albedo is the albedo without diffuse component in the incident irradiance, i.e. the illumination of the surface is from a single direction. It is defined as the integral of the BRDF over the reflection hemisphere of $2 \pi$ steradians (directional-hemispherical reflectance):

$$
A_{\mathrm{bs}}\left(\theta^{\prime}, \lambda\right)=\int_{0}^{2 \pi} \int_{0}^{\frac{\pi}{2}} R_{\mathrm{g}}\left(\theta^{\prime}, \varphi^{\prime} ; \theta, \varphi\right) \cos \theta \sin \theta \mathrm{d} \theta \mathrm{d} \varphi .
$$

In the particular case when there is only a diffuse isotropic component in the incident irradiance, the white-sky albedo can be defined as the integral of the surface BRDF over both the incident and reflection hemispheres (bi-hemispherical reflectance):

$$
A_{\mathrm{ws}}(\lambda)=\int_{0}^{2 \pi} \int_{0}^{\frac{\pi}{2}} \int_{0}^{2 \pi} \int_{0}^{\frac{\pi}{2}} R_{\mathrm{g}}\left(\theta^{\prime}, \varphi^{\prime} ; \theta, \varphi\right) \cos \theta^{\prime} \sin \theta^{\prime} \cos \theta \sin \theta \mathrm{d} \theta \mathrm{d} \varphi \mathrm{d} \theta^{\prime} \mathrm{d} \varphi^{\prime} .
$$

The so-called blue-sky albedo is a linear combination of $A_{\mathrm{bs}}$ and $A_{\mathrm{ws}}$ weighted by the fraction of diffuse skylight. The use of either $A_{\mathrm{bs}}, A_{\mathrm{ws}}$ or $A_{\mathrm{LER}}$ in different applications depends on the assumptions of each parameter and the particular application. In the NIR, the diffuse component in the radiation field is much smaller than the direct component so the use of the $A_{\mathrm{bs}}$ is justified because it assumes only direct light. $A_{\mathrm{ws}}$ might be more suitable for applications in the $\mathrm{UV} /$ visible spectral range where the diffuse component of the incident light may be of comparable size as the direct component. In any case, MODIS visible and NIR $A_{\mathrm{ws}}$ and $A_{\mathrm{bs}}$ do not differ on average more than $5 \%$ in summer (Oleson et al., 2003). $A_{\mathrm{ws}}$ is constant with solar incident direction, so its use is valid as a $A_{\text {LER }}$ but it accounts for some surface BRDF effects (Eq. 4.3).

Several models have been developed to describe surface BRDF (Wanner et al., 1995). These are either physical, empirical or semi-empirical models. Physical models are constructed using laws of physics to explicitly describe the processes that lead to the anisotropic behaviour of surface reflectance. Empirical models characterize the BRDF using mathematical functions that are suitable to describe the observed surface reflectance. Semi-empirical models describe the surface BRDF as a weighted sum of empirical functions derived from physical approximations.

Semi-empirical models are commonly used for global surface BRDF characterization using remote sensing instruments. In these models, surface reflectance is represented as linear combination of different terms (the so-called kernels) that characterize different types of scattering that lead to the directional signatures on the reflectance (Roujean et al., 1992). Typically these terms consist of an isotropic term, a volume scattering term, and a geometric scattering term. The weights of the kernels cannot be directly 
interpreted as physical characteristics from the reflecting surface, but just as a first order approximation of the structure of the surface BRDF (Gao et al., 2003).

In the semi-empirical BRDF Ross Thick-Li Sparse (hereinafter Ross-Li) kernel-driven model, the surface reflectance is expressed as a sum of an isotropic term and two kernels $\left(K_{\mathrm{i}}\right)$ that depend on incident zenith angle $\left(\theta^{\prime}\right)$, viewing zenith angle $(\theta)$ and relative azimuth angle $\left(\varphi-\varphi^{\prime}\right)$ :

$$
R_{\mathrm{g}}\left(\theta, \theta^{\prime}, \varphi-\varphi^{\prime}, \lambda\right)=f_{\mathrm{iso}}(\lambda)+f_{\mathrm{vol}}(\lambda) K_{\mathrm{vol}}\left(\theta, \theta^{\prime}, \varphi-\varphi^{\prime}\right)+f_{\mathrm{geo}}(\lambda) K_{\mathrm{geo}}\left(\theta, \theta^{\prime}, \varphi-\varphi^{\prime}\right)
$$

In Eq. $4.4 K_{\mathrm{i}}$ are the kernels from the semi-empirical Ross-Li model that describe the three basic scattering types and $f_{\mathrm{i}}$ are the surface BRDF parameters that are retrieved from surface reflectance observations from satellite measurements (e.g. MODIS). The surface reflectance cloud-free observations used to obtain the surface BRDF parameters are corrected for absorption and scattering by atmospheric gases, aerosols and thin clouds (Vermote et al., 1997). Improvements in the atmospheric correction scheme include as much information as possible derived from the satellite itself (e.g. MODIS aerosol optical thickness) (Vermote and Kotchenova (2008)).

The isotropy parameter $\left(f_{\text {iso }}\right)$ represents the isotropic scattering from a nadir incident and nadir view position. The volumetric scattering is represented by the Ross-Thick kernel, $K_{\mathrm{vol}}$. It is derived in a single scattering approximation from radiative transfer theory for a thick homogeneous layer of small scatterers, with equal reflectance and transmittance (Roujean et al., 1992). To account for the reflectance peak in the backscatter direction (i.e. the hot-spot effect) we include the modification on the volumetric kernel by Maignan et al. (2004). The geometric scattering is represented by the Li-Sparse kernel, $K_{\text {geo }}$. For this case, the scene is assumed to contain sparse objects that cast perfectly black shadows with sunlit and shaded portions of the ground and crown contributing to the modelled reflectance of the scene (Li and Strahler, 1986). The exact formulae for these kernels are summarized in Sect. S4.1.

\subsubsection{Surface BRDF implementation in DAK}

The radiative transfer model DAK (Doubling-Adding KNMI, Lorente et al. (2017), Stammes et al. (1989), de Haan et al. (1987)) is used in the GOME-2 and OMI cloud retrievals (FRESCO and OMCLDO2) and in the DOMINO $\mathrm{NO}_{2}$ retrieval. Originally DAK only considered Lambertian surfaces. To account for the surface reflectance anisotropy in DAK, we have implemented the Ross-Li kernel-driven model. We chose this model for consistency with the retrieval algorithm of the MODIS BRDF/Albedo product that we will use in our simulations. The MODIS satellite provides a reliable surface BRDF product and its resolution is suitable to capture surface anisotropy variations for OMI 
and GOME-2 resolution. MODIS BRDF/Albedo products have been successfully used in different fields of atmospheric and climate science such as analysis of radiative forcing due to vegetation change (Myhre et al., 2005) and assessment of land surface albedo in global climate models (Oleson et al., 2003).

After the implementation of the Ross-Li surface BRDF model in DAK, the surface reflectance matrix $R_{\mathrm{g}}$ now contains the full reflection properties of the surface and substitutes the constant isotropic value used for the Lambertian case. This matrix is filled with the surface reflectance calculated with the Ross-Li BRDF model via Eq. 4.4 as a function of $\theta, \theta^{\prime}$ for a specific $\varphi-\varphi^{\prime}$. For the Lambertian case, the matrix only contains the $(1,1)$ element, which is the value of the surface albedo. We neglect polarization in the BRDF. In the Doubling-Adding method for radiative transfer calculation, all the matrices (scattering, reflection and transmission matrices) are expanded in a Fourier series for the integration over $\varphi-\varphi^{\prime}$ following the approach in de Haan et al. (1987). For each Fourier term the Doubling-Adding procedure is applied separately, including the addition of the surface. The $m$-th Fourier coefficient matrix for the surface reflectance matrix is obtained from the relation:

$$
R_{\mathrm{g}}^{m}\left(\mu, \mu^{\prime}\right)=\frac{1}{2 \pi} \int_{0}^{2 \pi} d\left(\varphi-\varphi^{\prime}\right) \cos m\left(\varphi-\varphi^{\prime}\right) R_{\mathrm{g}}\left(\mu, \mu^{\prime}, \varphi-\varphi^{\prime}\right)
$$

where $\mu, \mu^{\prime}$ are the cosines of the zenith angles in the scattered and incident direction $\left(\theta, \theta^{\prime}\right)$ respectively and $\varphi-\varphi^{\prime}$ is the difference between the scattered and incident azimuth angles.

The coefficients of the Fourier expansion (Eq. 4.5) are calculated with the GaussLegendre quadrature integration method. It is possible to apply this method because the surface reflection matrix $R_{\mathrm{g}}$ is known at a certain number of division points $\varphi-\varphi^{\prime}$. The number of Fourier terms $(m)$ and Gaussian points for azimuth $\left(\varphi-\varphi^{\prime}\right)$ integration needed to resolve the surface BRDF shape depends on the illumination and viewing geometry. For geometries close to the hot-spot region in the backscatter direction, the number of Fourier terms and Gaussian points needed to reproduce the original BRDF increases significantly with respect to geometries outside the hot-spot. In order to reach an accuracy of $10^{-3}$ (difference between the original BRDF and the reconstructed BRDF with the Fourier expansion) over the hot spot region, 720 Gaussian points are needed for the azimuthintegration, and 300 Fourier terms. Outside the hot-spot region, using 60 Gaussian points and 30 Fourier terms in DAK reproduces the original surface reflectance values with an accuracy higher than $10^{-5}$. In the final implementation of the surface BRDF in the DAK RTM and in order to have an optimal simulation time, we used 100 Fourier terms and 360 Gaussian points for $\varphi-\varphi^{\prime}$, also over the hot-spot. The overall accuracy obtained with these numbers is within the errors of the radiative transfer modelling for application in 
satellite retrievals (Lorente et al., 2017).

retrievals from GOME-2 and OMI

One of the disadvantages of empirical models is that they depend on observations to derive the parameters $f_{\mathrm{i}}$ that describe the surface BRDF. Kernel based semi-empirical models like the Ross-Li model implemented in DAK only describe the surface reflection accurately for the range of illumination and viewing geometries of the measurements from which they have been derived (Litvinov et al., 2011). The geometries for which the semi-empirical models are valid are thus limited by extreme geometries of instruments like MODIS, which are typically 60-70 degrees for $\theta$ and $\theta^{\prime}$. This limit overlaps well with the $\theta$, $\theta^{\prime}$ values in OMI and GOME-2 measurements for which clouds and trace gas columns are retrieved. For geometries outside the range of MODIS measurement geometries, surface reflectance variations need to be extrapolated.

In radiative transfer modelling with the Doubling-Adding method, in order to calculate the radiation field correctly, the reflectance and transmittance values are needed for all $\theta, \theta^{\prime}$ between 0-90 degrees to integrate over the complete hemisphere. However, after implementing the Ross-Li BRDF model in DAK, surface reflectance was negative or too high for some combinations of extreme geometries. In order to avoid these unphysical values, we tried various ways of extrapolating values from valid (MODIS-range) angles to the more extreme angles. The different methods did not affect TOA reflectance and albedo values by more than $1 \%$. Finally, we constrained the surface reflectance to the range $[0,1]$ as in Eq. 4.6. This is reasonable as negative surface reflectance values are not physically valid and in nature surfaces with reflectance higher than 1 do not usually occur (except e.g. for surfaces covered by snow or ice). Therefore,

$$
\begin{array}{ccc}
\text { If } R_{\mathrm{g}}\left(\mu_{\mathrm{i}}, \mu_{\mathrm{i}}^{\prime}, \varphi_{\mathrm{i}}-\varphi_{\mathrm{i}}^{\prime}\right)<0 & \text { then } & R_{\mathrm{g}}\left(\mu_{\mathrm{i}}, \mu_{\mathrm{i}}^{\prime}, \varphi_{\mathrm{i}}-\varphi_{\mathrm{i}}^{\prime}\right)=0 \\
\text { If } R_{\mathrm{g}}\left(\mu_{\mathrm{i}}, \mu_{\mathrm{i}}^{\prime}, \varphi_{\mathrm{i}}-\varphi_{\mathrm{i}}^{\prime}\right)>1 \quad \text { then } \quad & R_{\mathrm{g}}\left(\mu_{\mathrm{i}}, \mu_{\mathrm{i}}^{\prime}, \varphi_{\mathrm{i}}-\varphi_{\mathrm{i}}^{\prime}\right)=1 .
\end{array}
$$

Figure 4.4 is an example of the surface reflectance computed with the Ross-Li BRDF model for a surface with parameters $\left(f_{\text {iso }}, f_{\text {vol }}, f_{\text {geo }}\right)=(0.0399,0.0245,0.0072)$. This combination of $f_{\mathrm{i}}$ values are the spatially averaged parameters from MODIS (BRDF/Albedo product MCD43A1) Band 3 (459-479 nm) over Amazonia (Lat: 5N-10S, Lon: 60-70W) for March 2008. This representation of a vegetated surface will be used in all the simulations in Sect. 4.3. The backscatter direction corresponds to values of $\varphi-\varphi^{\prime}=180^{\circ}$ in the polar plot (Fig. 4.4b) and negative values of $\theta$ in the principal plane plot (Fig. 4.4a). In the backscatter direction, the surface reflectance is two times higher than in the forward scatter direction $\left(\varphi-\varphi^{\prime}=0^{\circ}\right.$ in Fig. $4.4 \mathrm{~b}$ and positive $\theta$ in Fig. 4.4a). In the backscatter direction the so-called hot-spot is clearly visible when $\theta=(-) 30^{\circ}=\theta^{\prime}$.

The surface reflectance dependence on geometry as shown in Fig. 4.4 does not exist when a constant isotropic albedo or surface LER is used. Using a constant albedo for 

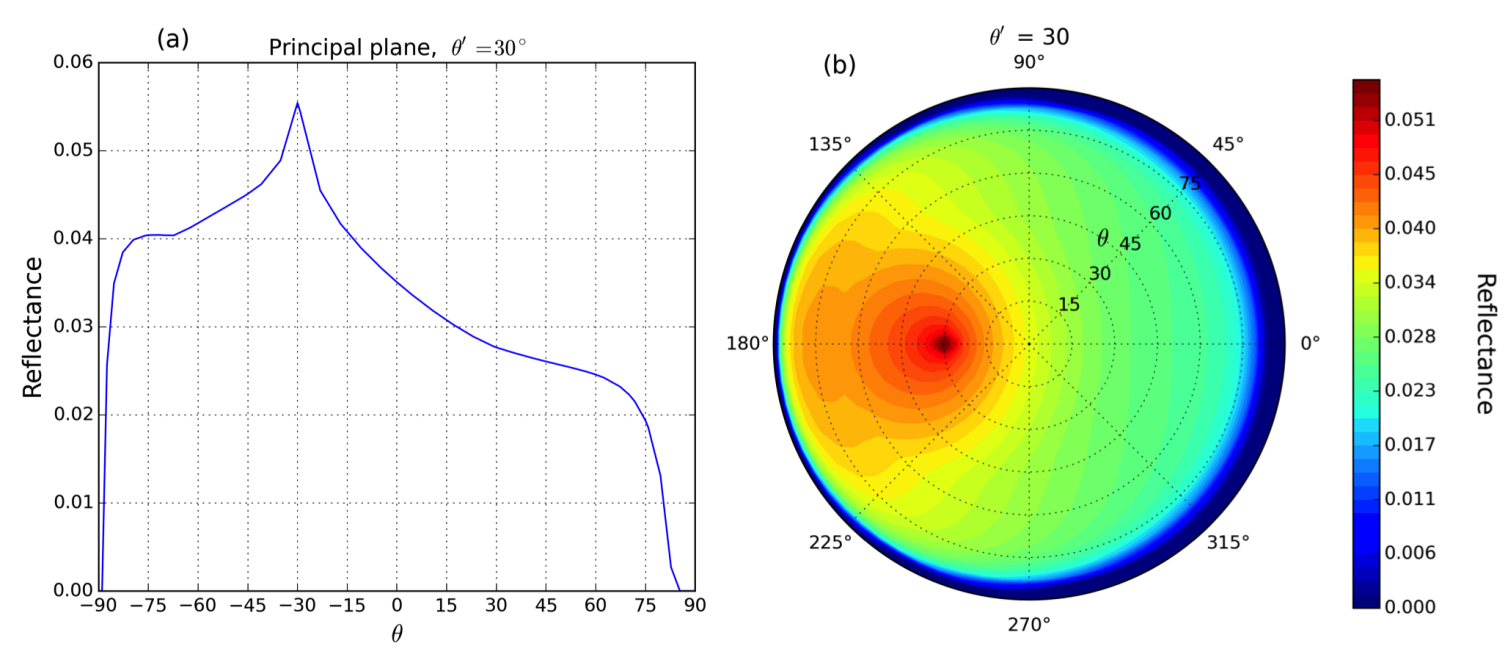

Figure 4.4: Surface reflectance modelled with the Ross-Li surface BRDF model with parameters from MODIS band $3(459-479 \mathrm{~nm})$ representing a vegetated surface over Amazonia (a) as function of viewing zenith angle in the principal plane $\left(\varphi-\varphi^{\prime}=180^{\circ}\right.$ for negative $\theta$ and $\varphi-\varphi^{\prime}=0^{\circ}$ for positive $\theta$ ) and (b) in a polar plot with $\varphi-\varphi^{\prime}$ along the azimuth axis and $\theta$ along the polar axis, for $\theta^{\prime}=30^{\circ}$.

this surface of 0.03 (the equivalent $\mathrm{A}_{\mathrm{ws}}$ is 0.034 ) means that in the backscatter direction the surface reflectance is underestimated. On the contrary, in the forward direction the surface reflectance is overestimated if an isotropic surface albedo is used. This surface reflectance difference between forward-backward scattering direction thus qualitatively explains the East-West bias in the surface LER in Fig. 4.1. West measurements in GOME2 correspond to the backscatter direction, and for this direction the surface reflectance is higher than in the forward scatter direction, i.e. East measurement in GOME-2. How this affects effective cloud fractions and $\mathrm{NO}_{2}$ AMFs from GOME-2 and OMI will be analysed in Sect. 4.4.

\subsubsection{Evaluation of surface BRDF effects in DAK TOA reflectances}

To evaluate the surface BRDF implementation in DAK we compare TOA reflectances with those simulated by other state-of-the-art radiative transfer models that include a description of surface BRDF effects. Both SCIATRAN (Rozanov et al., 2014) and LIDORT (Spurr, 2004) model the surface reflectance using the Ross-Li model. To minimize the differences due to factors other than the surface BRDF implementations itself, the settings of the three RTMs were as similar as possible. These settings include: no polarization, a plane parallel standard mid-latitude atmosphere and absorption by $\mathrm{O}_{3}, \mathrm{O}_{2}-\mathrm{O}_{2}$ and $\mathrm{NO}_{2}$.

We select two combinations of the Ross-Li BRDF parameters to model surface reflectance of different surfaces. We simulate TOA reflectances at two different wavelengths: 
469 and $645 \mathrm{~nm}$. These wavelengths correspond to the middle of the MODIS band 3 (459$479 \mathrm{~nm})$ and band $1(620-670 \mathrm{~nm})$, respectively.

Figure 4.5 shows the simulated TOA reflectance by DAK, SCIATRAN and LIDORT as a function of VZA along the principal plane at $469 \mathrm{~nm}$ and $645 \mathrm{~nm}$. The agreement between the models is within $0.5 \%$ for geometries outside the hot-spot region, and DAK and SCIATRAN agree within $1 \%$ over the hot-spot. With this simple validation we assure that our surface BRDF implementation in DAK is correct. The viewing geometry dependency of the TOA reflectance is the effect of Rayleigh scattering of a clean atmosphere. By comparing Fig. 4.4a with Fig. 4.5 we see that the effect of surface reflectance anisotropy is dampened by the scattering in the atmosphere but the hot-spot effect is still visible at both wavelengths after the radiation has passed through the atmosphere. Figure 4.5 also shows that the hot-spot effect is less prominent in TOA reflectance at 469 $\mathrm{nm}$ compared to $645 \mathrm{~nm}$, where the atmosphere is more transparent. At $469 \mathrm{~nm}$ Rayleigh scattering is stronger than at $645 \mathrm{~nm}$, reducing the effects of surface anisotropy on the TOA reflectance.
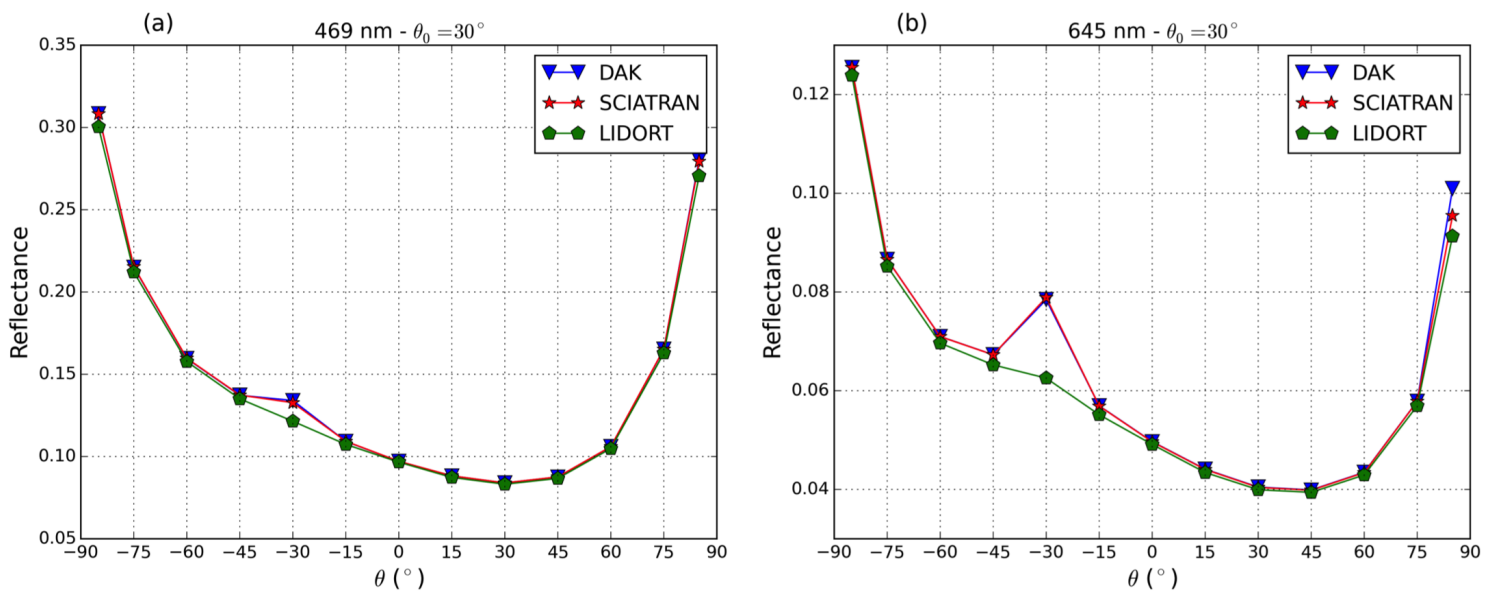

Figure 4.5: TOA reflectance at (a) $469 \mathrm{~nm}$ and (b) $645 \mathrm{~nm}$ simulated by DAK (blue), SCIATRAN (red) and LIDORT (green) with the Ross-Li surface BRDF model with parameters representing a vegetated surface over Amazonia as in Fig.4.4 as a function of the viewing zenith angle in the principal plane $\left(\varphi-\varphi_{0}=180^{\circ}\right.$ for negative $\theta$ and $\varphi-\varphi_{0}=0^{\circ}$ for positive $\theta$ ), for $\theta_{0}=30^{\circ}$.

We now compare TOA reflectance simulated with surface BRDF and TOA reflectance simulated assuming a Lambertian surface at $469 \mathrm{~nm}$ (Fig. 4.6a, b) and $758 \mathrm{~nm}$ (Fig. 4.6c, d). For simulations at $758 \mathrm{~nm}$ we use surface BRDF parameters from MODIS band 2 $(841-876 \mathrm{~nm})$ to account for the increase in surface reflectivity near $700 \mathrm{~nm}$ (the so-called red edge, e.g.Tilstra et al. (2017)). To test the representativeness of band 2 at $758 \mathrm{~nm}$, we scaled the parameters from band $3(459-479 \mathrm{~nm})$ using the ratio of reflectance at $772 \mathrm{~nm}$ and $469 \mathrm{~nm}$, and the differences with the parameters from MODIS band 2 were negligible. 

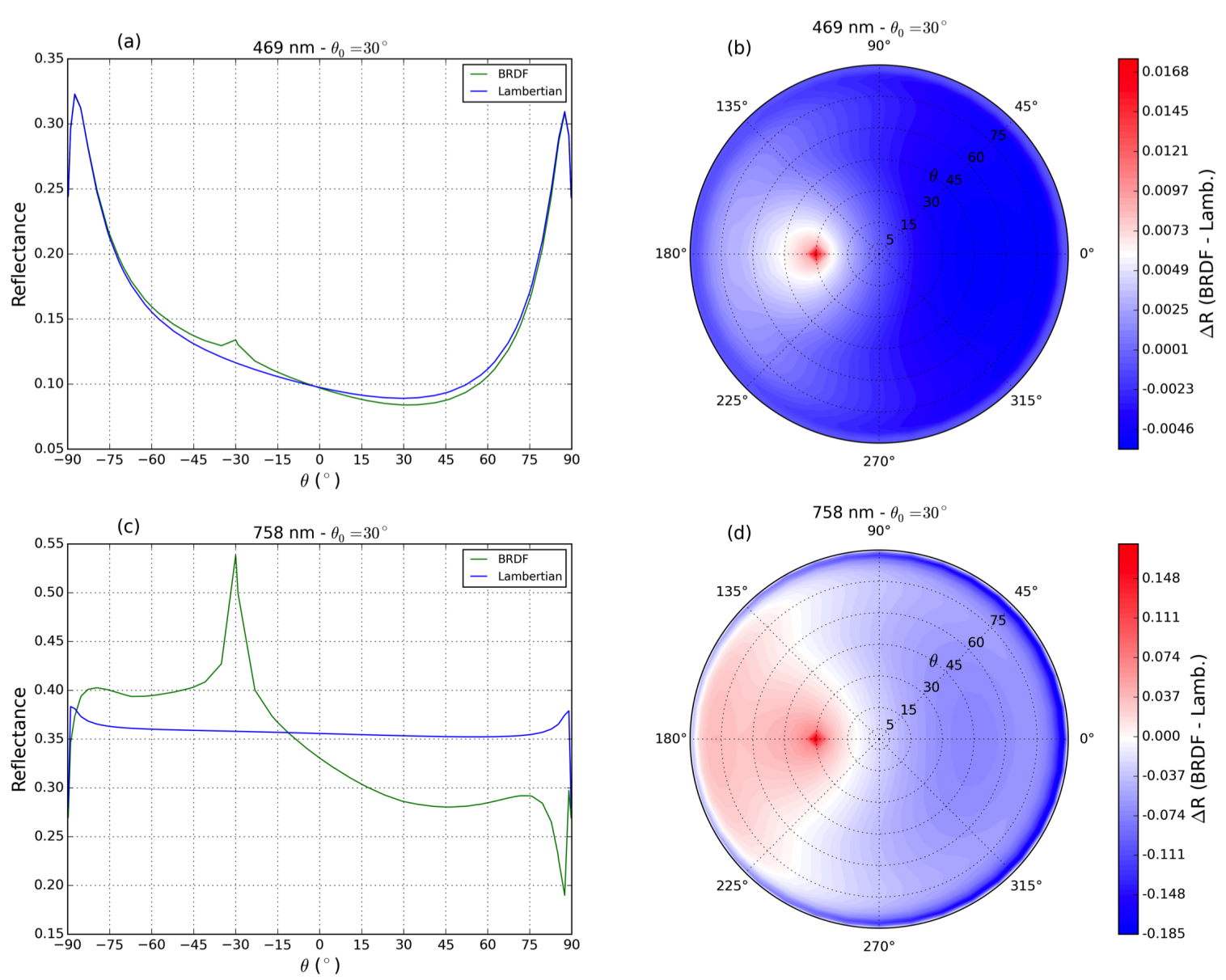

Figure 4.6: (a, c) TOA reflectance as a function of viewing zenith angle simulated by DAK at $469 \mathrm{~nm}$ and $758 \mathrm{~nm}$ with a Lambertian surface (blue line) and with surface BRDF (green line) in the principal plane $\left(\varphi-\varphi_{0}=180^{\circ}\right.$ for negative $\theta$ and $\varphi-\varphi_{0}=0^{\circ}$ for positive $\theta$ ). (b, d) Absolute differences between TOA reflectance with surface BRDF and with a Lambertian surface at $469 \mathrm{~nm}$ and $758 \mathrm{~nm}$. Surface BRDF parameters represent a vegetated surface over Amazonia at $469 \mathrm{~nm}\left(f_{\text {iso }}, f_{\text {vol }}, f_{\text {geo }}\right)=(0.0399,0.0245,0.0072)$ and $758 \mathrm{~nm}\left(f_{\text {iso }}, f_{\text {vol }}, f_{\text {geo }}\right)$ $=(0.36,0.24,0.03)$. Note the different scales.

For the Lambertian case, we use the equivalent $A_{\mathrm{ws}}$ calculated using the surface BRDF model with Eq. 4.3. Figure 4.6b shows that at $469 \mathrm{~nm}$, the highest absolute differences are around the hot-spot region in the backward scattering direction, where TOA reflectance with BRDF is up to $15 \%$ higher than the TOA reflectance for the Lambertian case. In the forward scatter direction, TOA reflectance with surface BRDF is 7\% lower than for the Lambertian surface. At $758 \mathrm{~nm}$ the effect of surface reflectance anisotropy is much stronger than at $469 \mathrm{~nm}$ (Fig. 4.6c). The highest absolute differences at $758 \mathrm{~nm}$ (Fig. $4.6 \mathrm{~d}$ ) are in the hot-spot region in the backscatter direction (up to 30\%) and for very extreme angles $\left(\theta>85^{\circ}\right)$ in the forward scattering direction.

These results are consistent with those shown in Fig. 4.1 and Fig. 4.2: for GOME- 
2A West measurements (backscatter direction), TOA reflectances with surface BRDF are higher than for the Lambertian surface. If these differences are not accounted for in the cloud retrieval, cloud fractions will be biased high in the West measurements to match the measured TOA reflectance. For East measurements in the forward scatter direction, TOA reflectance with surface BRDF is lower than for the Lambertian surface. Cloud fractions retrieved with surface LER will be biased low to match the measured TOA reflectance. Results from Fig. 4.6 underline that surface BRDF effects in retrieved cloud fractions are stronger for FRESCO at $758 \mathrm{~nm}$ than for OMCLDO2 at $477 \mathrm{~nm}$. Our results also show that the error in TOA reflectances due to the use of a Lambertian albedo is substantial, but its magnitude is highly dependent on the spectral and geometrical characteristics of the measurements: effects are stronger at $758 \mathrm{~nm}$ and around the hot-spot region in the backscatter direction.

\subsection{Role of surface BRDF in cloud retrievals}

\subsubsection{Synthetic cloud fraction retrieval}

In Sect. 4.2 we showed that there is an East-West bias in the retrieved cloud fractions from GOME-2 and OMI. Effective cloud fractions in FRESCO and OMCLDO2 are retrieved as follows (Stammes et al. (2008), Veefkind et al. (2016)):

$$
c_{\mathrm{eff}}=\frac{R_{\mathrm{meas}}-R_{\mathrm{cr}}}{R_{\mathrm{cd}}-R_{\mathrm{cr}}} .
$$

$R_{\text {meas }}$ is the TOA reflectance measured by the satellite instrument, $R_{\text {cr }}$ is the simulated clear-sky TOA reflectance, and $R_{\mathrm{cd}}$ is the simulated cloudy-sky TOA reflectance assuming that the cloud is a Lambertian reflector with a fixed albedo of 0.8. In the current versions of FRESCO (v7) and OMCLDO2 (v2.0) cloud retrievals, the simulated clear-sky TOA reflectances in Eq. 4.7 assume that the surface is Lambertian. Due to this assumption, any surface anisotropy signal in the measured TOA reflectance $\left(R_{\text {meas }}\right)$ is neglected, and, consequently ends up in the retrieved effective cloud fraction.

To improve our understanding of how surface reflectance anisotropy influences the retrieval of cloud fractions, we use the forward model DAK to approximate $R_{\text {meas }}$ by simulating the TOA reflectance for a scene with a Henyey-Greenstein cloud and surface reflectance anisotropy. This resembles what the satellite would measure in a realistic cloudy scene. We express $R_{\text {meas }}$ as the sum of TOA reflectance of the cloudy and the clear parts of the scene, weighted by a geometric cloud fraction $c_{\text {geo }}$ (independent pixel approximation):

$$
R_{\text {meas }} \approx R_{\text {sim }}=c_{\text {geo }} \cdot R_{\mathrm{cd}}+\left(1-c_{\text {geo }}\right) R_{\mathrm{cr}}
$$


The effective cloud fraction is the part of the pixel that the Lambertian cloud has to occupy to match the observed reflectance. The geometric cloud fraction is the part of the pixel that is covered by the "true" cloud (Stammes et al., 2008).

The settings of the simulations are summarized in Table 4.1. We simulate TOA reflectances and cloud fractions in the spectral regions where cloud fractions are calculated in the cloud algorithms. The Ross-Li BRDF parameters are from a climatology created by the QA4ECV Land Group at the Mullard Space Science Laboratory (University College London). This dataset consists of daily BRDF parameters collected from 16 years of MODIS measurements (Strahler et al. (1999), MCD43A1) from 2000 to 2016 (QA4ECVWP4, 2016). Parameters from band $3(459-479 \mathrm{~nm})$ are representative for simulations in the $\mathrm{O}_{2}-\mathrm{O}_{2}$ absorption band and parameters from band 2 (841-876 $\mathrm{nm}$ ) for simulations in the $\mathrm{O}_{2}$-A band (see Sect. 4.3.2). Monthly averaged parameters from this dataset over Amazonia are shown in Fig. S4.2 and S4.3 in the supplementary material.

We calculate cloud fractions using Eq. 4.7 with $R_{\text {cr }}$ simulated with a Lambertian surface consistent with the current $\mathrm{O}_{2}-\mathrm{O}_{2}$ and FRESCO+ retrievals (hereafter Lambertian $c_{\text {eff }}$ ) and by accounting for surface BRDF effects (hereafter BRDF $c_{\text {eff }}$ ). The Lambertian cloud is located at the same pressure level as the Henyey-Greenstein cloud so we can isolate surface BRDF effects on cloud fraction only (see settings in Table 1).

Table 4.1: Settings for Lambertian and BRDF $c_{\text {eff }}$ simulations in Sect. 4.4.1. Inverse model for Lambertian $c_{\text {eff }}$ reproduces current $\mathrm{O}_{2}-\mathrm{O}_{2}$ and FRESCO+ retrievals, with Lambertian surface and Lambertian cloud. Inverse model for BRDF $c_{\text {eff }}$ reproduces the retrieval accounting for surface BRDF effects.

\begin{tabular}{cc}
\hline \multicolumn{3}{c}{ Forward model, $R_{\text {meas }}$} \\
\hline Henyey-Greenstein scattering cloud & 0.85 \\
Asymmetry parameter, g & 30 \\
Cloud optical thickness, $\tau_{c}$ & $1-2 \mathrm{~km}$ \\
Cloud altitude & $0,0.05,0.2,0.5$ \\
Geometric cloud fraction, $c_{\text {geo }}$ & $\left.f_{\text {vol }}, f_{\text {geo }}\right)$ for $R_{\mathrm{cr}}$ \\
Surface reflectance: BRDF parameters & $0.03,0.02,0.01$ \\
$\lambda=477 \mathrm{~nm}$ & $0.4,0.25,0.08$ \\
$\lambda=758 \mathrm{~nm}$ & \\
\multicolumn{2}{c}{ Inverse model, $c_{\mathrm{eff}}$} \\
\hline Lambertian cloud $\left(R_{\mathrm{cd}}\right)$ & 0.8 \\
Cloud albedo, $\mathrm{A}_{\mathrm{cd}}$ & $1-2 \mathrm{~km}$ \\
Cloud altitude & 0.0217 \\
Lambertian $c_{\mathrm{eff}}:$ surface albedo $\left(\mathbf{A}_{\mathrm{ws}}\right)$ for & $R_{\mathrm{cr}}$ \\
$\lambda=477 \mathrm{~nm}$ & 0.337 \\
$\lambda=758 \mathrm{~nm}$ & $0.03,0.02,0.01$ \\
BRDF $c_{\mathrm{eff}}:$ surface parameters $\left(f_{\text {iso }}, f_{\mathrm{vol}}, f_{\mathrm{geo}}\right)$ for $R_{\mathrm{cr}}$ \\
$\lambda=477 \mathrm{~nm}$ & $0.4,0.25,0.08$ \\
$\lambda=758 \mathrm{~nm}$ &
\end{tabular}

Figure 4.7 shows that cloud fractions accounting for surface BRDF effects (dashed 

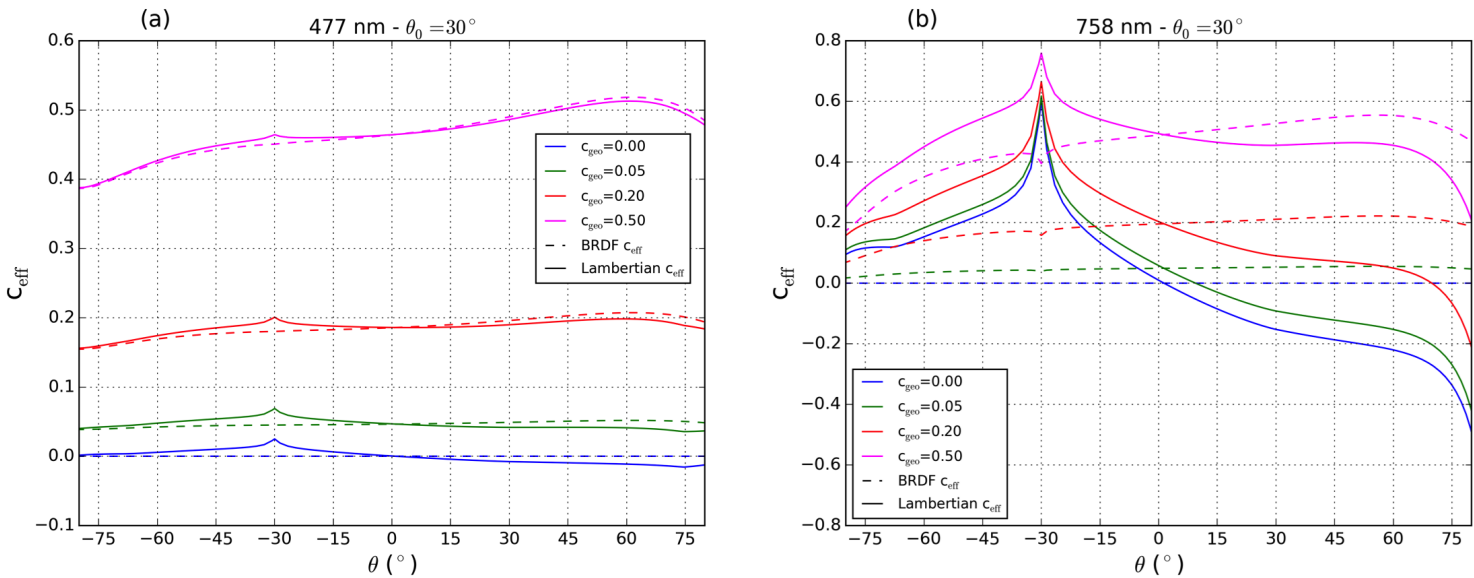

Figure 4.7: Simulated effective cloud fraction at (a) $477 \mathrm{~nm}$ and (b) $758 \mathrm{~nm}$ as a function of viewing zenith angle for different geometric cloud fractions along the principal plane $\left(\varphi-\varphi_{0}\right.$ $=180^{\circ}$ for negative $\theta$ and $\varphi-\varphi_{0}=0^{\circ}$ for positive $\left.\theta\right) . \theta_{0}=30^{\circ}$ and $c_{\text {geo }}=0,0.05,0.2$ and 0.5. At $477 \mathrm{~nm}$ : BRDF parameters (solid line) $\left(f_{\text {iso }}, f_{\mathrm{vol}}, f_{\text {geo }}\right)=(0.03,0.02,0.01)$ and for Lambertian surface $A_{\mathrm{ws}}=0.0217$ (dashed line). At $758 \mathrm{~nm}$ : BRDF parameters (solid line) $\left(f_{\text {iso }}, f_{\mathrm{vol}}, f_{\text {geo }}\right)=(0.4,0.25,0.08)$ and for Lambertian surface $A_{\mathrm{ws}}=0.337$ (dashed line).

lines) depend only weakly on geometry whereas cloud fractions with a Lambertian surface (solid lines) are higher in the backward scattering direction, at both $477 \mathrm{~nm}$ and $758 \mathrm{~nm}$ and especially for the lowest $c_{\text {geo }}$. At $758 \mathrm{~nm}$ this is true even for a geometric cloud fraction as high as 0.5. In the backward scattering direction, surface reflectance is higher than reflectance by a Lambertian surface. Therefore clear-sky TOA reflectance with a Lambertian surface cannot explain the higher simulated reflectance in Eq. 4.7, which results in high Lambertian $c_{\text {eff }}$.

Surface BRDF effects are more important for small cloud fractions, and less for large cloud fractions. For cloudy pixels the effect of surface reflectance anisotropy vanishes because the scattering by the cloud dominates in the measured reflectance. Fig. 4.7 shows that for large $c_{\text {geo }}$, the viewing zenith angle dependency of the effective cloud fractions is due to the Henyey-Greenstein scattering cloud, which gives relatively higher scattering in the forward direction.

At $477 \mathrm{~nm}$, surface BRDF effects on cloud fractions are less evident than at 758 $\mathrm{nm}$. In the visible spectral region, surface BRDF effects are suppressed by Rayleigh scattering smoothing out the surface anisotropy effects on TOA reflectances. For lower geometric cloud fraction, Lambertian cloud fractions are moderately higher (by 0.05) in the backscatter direction than in the forward scatter direction. These findings underscore the relevance of accounting for surface BRDF effects because measurements with small cloud fractions are most sensitive to pollution in the lower troposphere.

Differences in Lambertian cloud fractions between backward and forward scattering 
directions at $758 \mathrm{~nm}$ are on average 0.35. At $477 \mathrm{~nm}$, the differences amount to 0.1 , depending on the surface and the geometry. This is consistent with the observed bias in FRESCO and OMCLDO2 cloud fractions shown in Fig. 4.2. The absence of a backwardforward scattering dependency in the BRDF $c_{\text {eff }}$ implies that accounting for surface BRDF effects will reduce the East-West across-track bias in retrieved cloud fractions.

\subsubsection{GOME-2A cloud fraction simulations}

We simulate Lambertian and BRDF cloud fractions for GOME-2A measurements over Amazonia in March 2008. We use the exact illumination and viewing geometry $\left(\theta, \theta_{0}, \varphi-\right.$ $\varphi_{0}$ ) of each individual measurement and colocate MODIS pixels with the GOME-2A pixel centre to obtain the surface BRDF parameters over the scene. For Lambertian cloud fractions, we use the GOME-2A surface LER value from Tilstra et al. (2017). To simulate the measured reflectance, we assume a geometric cloud fraction distribution with an area-wide average of $\overline{c_{\text {geo }}}=0.33$. Figure $8 \mathrm{a}, \mathrm{d}$ show the $\overline{\mathrm{c}_{\text {geo }}}$ distribution for East and West measurements respectively.

Figure 4.8 shows simulated Lambertian (b,e) and BRDF (c,f) effective cloud fractions for East and West GOME-2A measurements. For East measurements, both Lambertian $\left(\overline{c_{\text {eff }}}=0.32\right)$ and $\operatorname{BRDF}\left(\overline{c_{\text {eff }}}=0.28\right)$ cloud fraction simulations capture the original geometric cloud fraction distribution and absolute values. This means that surface BRDF effects are weaker in the East, consistent with the smaller surface LER climatology bias for measurements on the East part of the orbit (Fig. 4.1). For West measurements, the original distribution is reproduced well in the BRDF cloud fractions $\left(\overline{c_{\text {eff }}}=0.29\right)$, but the Lambertian values show a significant overestimation $\left(\overline{c_{\mathrm{eff}}}=0.51\right)$. A box-plot of these distributions is shown in Fig. S4.4 in the supplementary material. The East-West bias in the Lambertian simulation (0.2) is considerably reduced (0.01) for the BRDF cloud fractions: the across-track bias in the FRESCO data is a direct consequence of neglecting surface BRDF effects. We conclude that accounting for these surface BRDF effects can largely solve the bias in cloud fractions measured in the backscatter regime over Amazonia. Although we have not made an analysis of the surface BRDF effects in a complete retrieval, the biases in cloud fraction found over other regions will probably be reduced after accounting for surface BRDF effects.

\subsection{Role of surface $\mathrm{BRDF}$ in $\mathrm{NO}_{2}$ retrievals}

Here we investigate the effects of accounting for surface reflectance anisotropy on tropospheric $\mathrm{NO}_{2}$ column retrievals under clear-sky and partly cloudy conditions. We calculate 
The importance of surface reflectance anisotropy for cloud and $\mathrm{NO}_{2}$ retrievals from GOME-2 and OMI

GOME-2A East/Forward scatter measurements

(a) Geometric cloud fraction

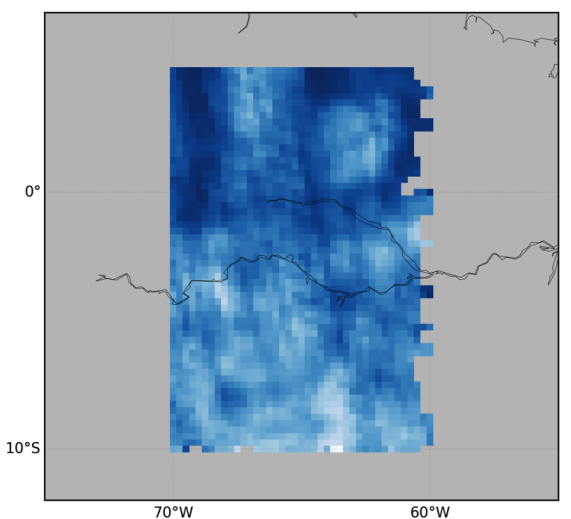

(b) Lambertian $C_{\text {eff }}$

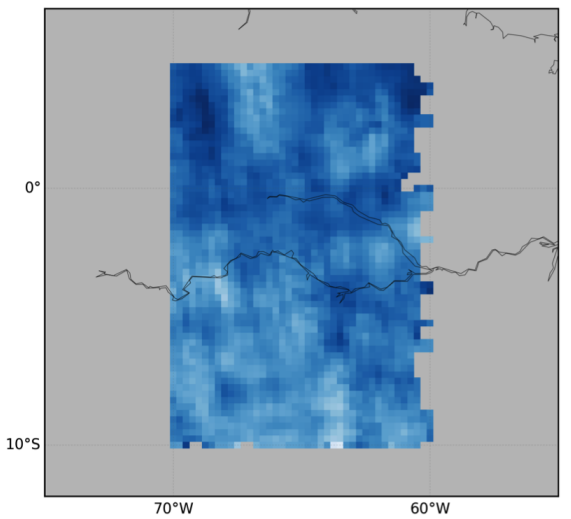

(c) BRDF $C_{\text {eff }}$

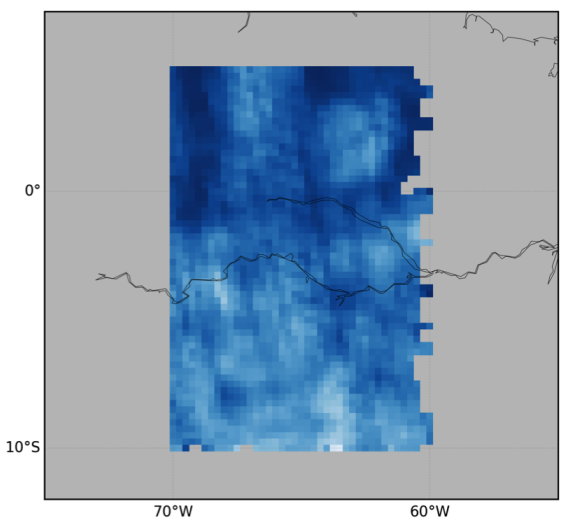

GOME-2A West/Backward scatter measurements

(d) Geometric cloud fraction

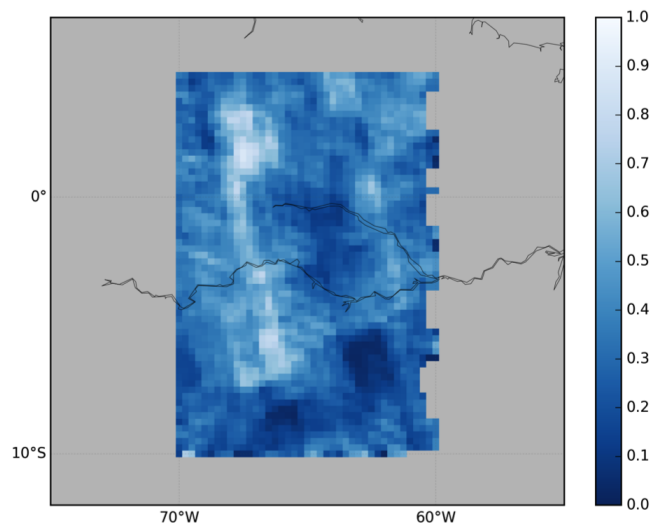

(e) Lambertian $C_{\text {eff }}$

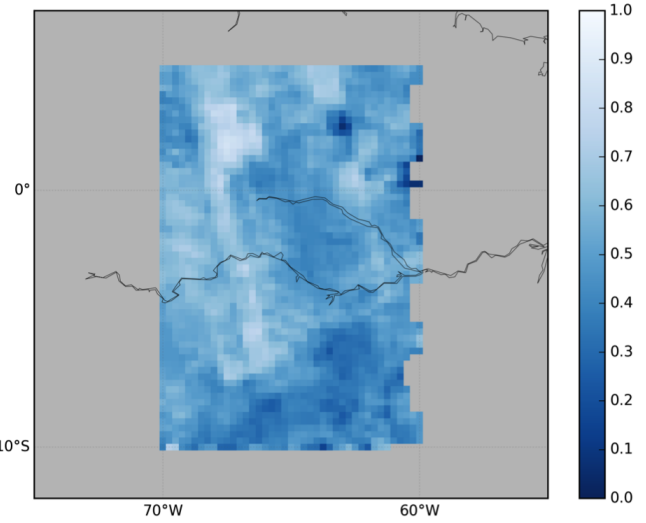

(f) BRDF $C_{\text {eff }}$

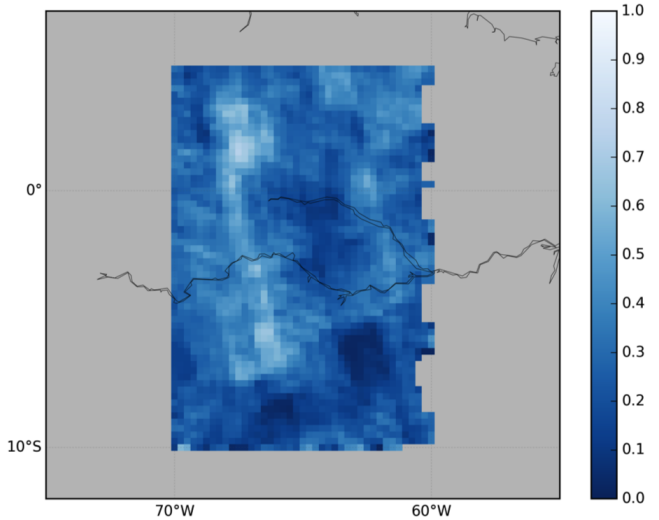

Figure 4.8: Geometric cloud fraction distributions for (a) East and (d) West GOME-2A measurements. (b, e) Lambertian and (c, f) BRDF cloud fraction simulations for East (first column) and West (second column) GOME-2A measurements. Plots show averaged cloud fractions in a $0.25^{\circ} \times 0.25^{\circ}$ grid over Amazonia (Lat: 5N-10S, Lon: 60-70W) for March 2008. 
tropospheric AMFs with surface BRDF and with a Lambertian surface, including the surface BRDF effects on retrieved cloud fractions (Sect. 4.4) for GOME-2A measurements over Amazonia and over France.

In satellite retrievals of trace gases, an air mass factor $(M)$ is used to convert the slant column density $\left(N_{\mathrm{s}}\right.$, SCD) from the measured reflectance spectra into the vertical column $\operatorname{density}\left(N_{\mathrm{v}}, \mathrm{VCD}\right)$ :

$$
M\left(R_{\mathrm{s}}, P_{\mathrm{s}}, f_{\mathrm{cd}}, P_{\mathrm{cd}}, \overrightarrow{\mathrm{x}_{\mathrm{a}}}, \theta, \theta_{0}, \varphi-\varphi_{0}\right)=\frac{N_{\mathrm{s}}}{N_{\mathrm{v}}}
$$

The AMF depends on the surface reflectance $\left(R_{\mathrm{s}}\right)$, surface pressure $\left(P_{\mathrm{s}}\right)$, cloud fraction and pressure $\left(f_{\mathrm{cd}}, P_{\mathrm{cd}}\right)$, a priori $\mathrm{NO}_{2}$ profile $\left(\overrightarrow{\mathrm{x}_{\mathrm{a}}}\right)$ and measurement geometry $\left(\theta, \theta_{0}, \varphi-\right.$ $\left.\varphi_{0}\right)$.

Here, tropospheric $\mathrm{NO}_{2}$ AMFs are calculated by differencing the logarithm of simulated TOA reflectances with and without trace gas in the troposphere divided by the absorption optical thickness of the gas $\tau_{\text {gas }}$ :

$$
M=-\frac{\ln R\left(\tau_{\text {gas }}\right)-\ln R\left(\tau_{\text {gas }}=0\right)}{\tau_{\text {gas }}} .
$$

AMF is directly affected by the assumption of a Lambertian surface instead of an anisotropic surface in the simulated TOA reflectance. In addition, AMFs are indirectly affected by the cloud radiance fraction $\left(f_{\text {cd }}\right)$ used to correct for residual clouds, in which calculation a Lambertian surface is assumed as well. To account for the presence of clouds, we use the independent pixel approximation (IPA, see also Eq. 4.8) which consists of calculating the total AMF for a partly cloudy scene as a linear combination of cloudy $\left(M_{\mathrm{cd}}\right)$ and clear $\left(M_{\mathrm{cr}}\right)$ components of the AMF, weighted by the cloud radiance fraction $w$ :

$$
M=w M_{\mathrm{cd}}+(1-w) M_{\mathrm{cr}} .
$$

The use of a Lambertian surface thus influences the AMF directly via $M_{\mathrm{cr}}$ and indirectly via $w$ :

$$
w=\frac{c_{\text {eff }} R_{\mathrm{cd}}}{c_{\mathrm{eff}} R_{\mathrm{cd}}+\left(1-c_{\mathrm{eff}}\right) R_{\mathrm{cr}}}
$$

where $c_{\text {eff }}$ is the cloud fraction and $R_{\mathrm{cd}}, R_{\mathrm{cr}}$ are the radiances for a totally cloudy and clear-sky scene, respectively. Cloud radiance fraction depends on the Lambertian surface assumption via $R_{\mathrm{cr}}$ and $c_{\mathrm{eff}}$. 


\subsubsection{BRDF effects on tropospheric $\mathrm{NO}_{2}$ air mass factors}

We calculate AMFs with Eq. 4.10 by simulating TOA reflectances with surface BRDF and with a Lambertian surface. The settings of the simulations are summarized in Table 4.2. Based on our analysis (Sect. 4.4.1), we include a change of \pm 0.05 over the Lambertian cloud fraction with a decrease of -0.05 in the backward scattering direction and an increase of +0.05 in the forward scattering direction, to quantify how the surface BRDF effects on clouds propagate to the final AMF.

Table 4.2: Settings for the Lambertian and BRDF tropospheric $\mathrm{NO}_{2}$ AMF calculations shown in Fig. 9.

\begin{tabular}{|c|c|}
\hline & Lambertian AMF \\
\hline Surface parameters & $\left(f_{\text {iso }}, f_{\mathrm{vol}}, f_{\mathrm{geo}}\right)=(0.04,0.03,0.008)$ \\
\hline Cloud fraction & $\begin{array}{l}\text { Backward scat.: } c_{\text {eff }}(\mathrm{BRDF})=0.1-0.05 \\
\text { Forward scat.: } c_{\text {eff }}(\mathrm{BRDF})=0.1+0.05\end{array}$ \\
\hline Cloud radiance fraction & $R_{\mathrm{cr}}, R_{\mathrm{cd}}, c_{\mathrm{eff}}(\mathrm{BRDF})$ \\
\hline & Common settings \\
\hline Atmospheric profile & $\begin{array}{l}\text { Mid latitude standard atmosphere } \\
\text { Surface pressure: } \mathrm{P}_{s}=1013 \mathrm{hPa}\end{array}$ \\
\hline $\mathrm{NO}_{2}$ tropospheric column & $\begin{array}{l}\text { Moderately polluted: } N_{\mathrm{v}, \text { trop }}=4 \cdot 10^{15} \text { molec } \cdot \mathrm{cm}^{-2} \\
\text { Unpolluted: } N_{\mathrm{v}, \text { trop }}=0.2 \cdot 10^{15} \text { molec } \cdot \mathrm{cm}^{-2}\end{array}$ \\
\hline Lambertian cloud (IPA) & $\begin{array}{l}\text { Cloud albedo: } A_{\mathrm{cd}}=0.8 \\
\text { Cloud pressure: } P_{\mathrm{cd}}=850 \mathrm{hPa}\end{array}$ \\
\hline
\end{tabular}

Figure 4.9a shows surface BRDF effects on $M_{\mathrm{cr}}$ for a moderately polluted troposphere as a function of VZA along the principal plane. In the backward scattering direction, BRDF $M_{\text {cr }}$ is higher by $5-20 \%$. The higher surface BRDF reflectance and TOA reflectance makes the retrieval more sensitive to the $\mathrm{NO}_{2}$ in the boundary layer. In the forward scattering direction, BRDF $M_{\mathrm{cr}}$ is lower by $5-15 \%$.

Figure 4.9b shows surface BRDF effects on $w$ and Fig. 4.9c shows the combined effect on total tropospheric $M$ of changes in $M_{\text {cr }}$ and in $w$ in a partly cloudy scene with a $\mathrm{c}_{\mathrm{eff}}($ Lamb. $)=0.1$. In the backward scattering direction, tropospheric $M$ is $9-30 \%$ higher when accounting for surface BRDF effects. The decrease in $\mathrm{c}_{\text {eff }}$ (and hence $w$ ) makes the retrieval more sensitive to the $\mathrm{NO}_{2}$ below the cloud. In the forward scattering direction, tropospheric $M$ is $14-22 \%$ lower when accounting for surface BRDF effects because of the stronger screening effect by the higher BRDF $c_{\text {eff }}$. The surface BRDF effect on $w$ enhances the effect on the clear-sky AMF by up to $10 \%$ in both the forward and backward scattering direction.

Figure 4.10 shows surface BRDF effects on total tropospheric $M$ in partly cloudy scenes with increasing cloudiness for the specific combination of $\left(\theta, \theta_{0}\right)=\left(45^{\circ}, 30^{\circ}\right)$ in the principal plane, representative of typical GOME-2 or OMI measurements. For a polluted troposphere (represented by stars), the total effect of accounting for surface BRDF is 

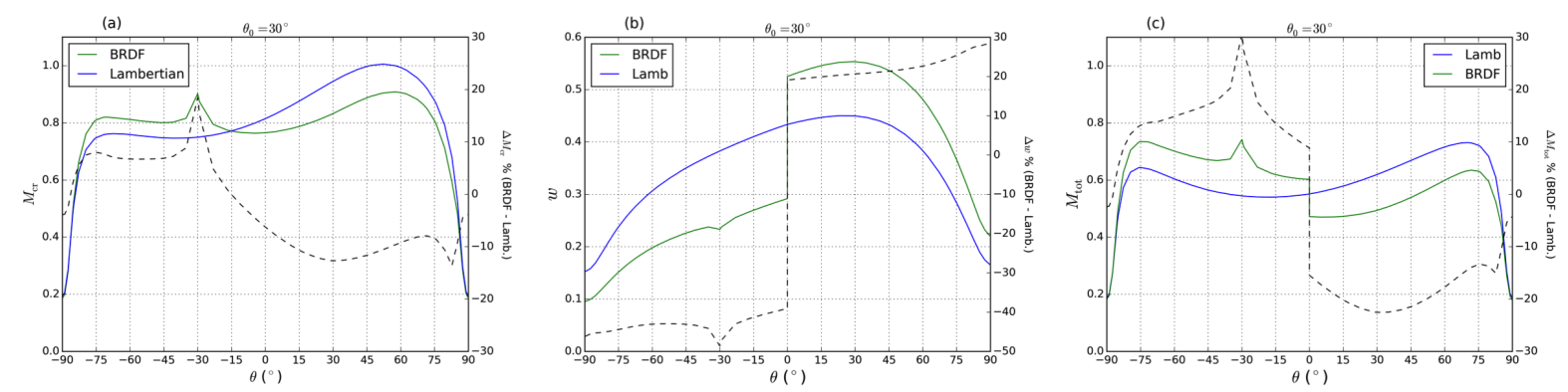

Figure 4.9: (a) Clear-sky tropospheric $\mathrm{NO}_{2} \mathrm{AMF}$, (b) cloud radiance fraction and (c) total tropospheric $\mathrm{NO}_{2}$ AMF computed with surface BRDF (green) and with a Lambertian surface (blue) and their relative difference (right axis) as a function of viewing zenith angle in the principal plane $\left(\varphi-\varphi_{0}=180^{\circ}\right.$ for negative $\theta$ and $\varphi-\varphi_{0}=0^{\circ}$ for positive $\left.\theta\right)$, for $\theta_{0}=30^{\circ}$. $\mathrm{P}_{\mathrm{cd}}$ $=850 \mathrm{hPa}$, Lambertian $\mathrm{c}_{\text {eff }}=0.1$ and BRDF $c_{\text {eff }}=0.10 \pm 0.05$. Surface BRDF parameters are $\left(f_{\text {iso }}, f_{\mathrm{vol}}, f_{\text {geo }}\right)=(0.04,0.03,0.008)$ and $\mathrm{A}_{\mathrm{ws}}=0.036$ for the Lambertian surface. Troposphere is moderately polluted $\left(\mathrm{N}_{\mathrm{v}, \text { trop }}=4 \cdot 10^{15} \mathrm{molec} \cdot \mathrm{cm}^{-2}\right)$.

to increase $M$ in the backward scattering direction (Fig. 4.10a) and to reduce $M$ in the forward scattering direction (Fig. 4.10b). Average relative differences of about $15 \%$ for $M_{\text {cr }}$ increase up to $25-40 \%$ for low cloud fractions (below 0.1) (see Fig. S4.5 in the supplementary material). The effect decreases for higher cloud fractions, with relative differences below $10 \%$ for cloud fractions higher than 0.5 . For a lower bias in $c_{\text {eff }}$ (e.g. 0.02), the effect on $M$ reduces to about $15-20 \%$, which is still a considerable effect.

For an unpolluted troposphere, surface BRDF effects on $M_{\mathrm{cr}}$ are in the same direction as in the polluted case but smaller $(-7$ to $+11 \%)$. In a partly cloudy scene, Lambertian and BRDF tropospheric $M$ are similar within -3 to $7 \%$. Because there is very little $\mathrm{NO}_{2}$ below the cloud, the effect of change in $w$ counteracts (and largely cancels) the effect on the clear-sky AMF (circles in Fig. 4.10).

Figure 4.11 shows surface BRDF effects on total tropospheric AMF as a function of cloud pressure, for a cloud fraction of 0.1. For cloud pressures higher than the $850 \mathrm{hPa}$ assumed in Fig. 4.10, the contribution of surface BRDF effects to the change in $M$ from the change in cloud fractions is dampened. There is a range of cloud pressures (in Fig. 4.11 between 900 and $950 \mathrm{hPa}$ ) for which the effects on $M_{\mathrm{cr}}$ and on cloud fraction compensate each other. For an even higher cloud pressure (e.g. $978 \mathrm{hPa}$ ), $M_{\mathrm{cd}}$ is larger than $M_{\mathrm{cr}}$ and the sign of the effect changes. In the backward scattering we have lower BRDF AMFs and in the forward scattering higher BRDF AMFs. In the unpolluted situations the differences become larger for higher cloud pressures.

Although this study does not address surface BRDF effects on cloud pressure, we did a preliminary analysis applying a directional surface LER derived from GOME-2 in FRESCO+. The analysis shows that accounting for surface reflectance anisotropy effects reduces the cloud pressure by $40 \mathrm{hPa}$ on average (with differences up to $120 \mathrm{hPa}$ ). This 

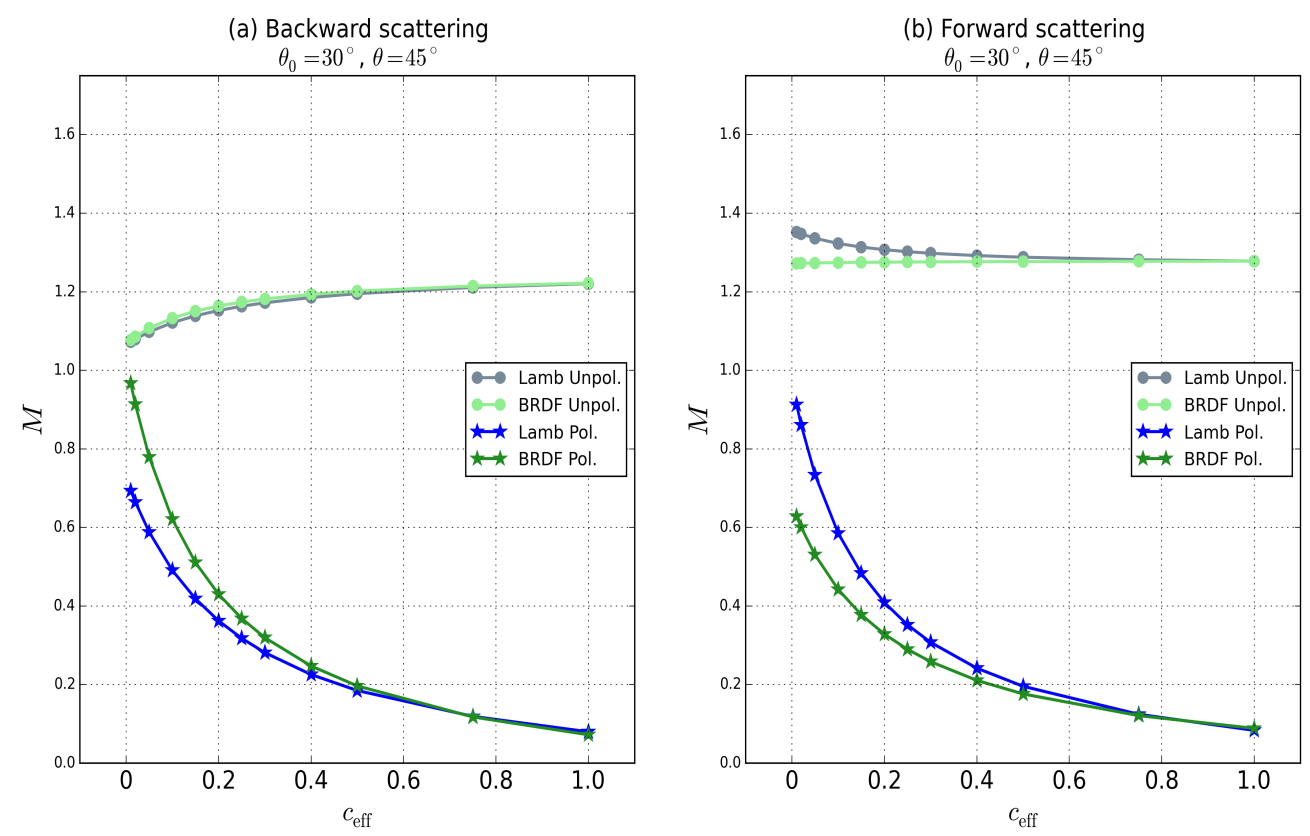

Figure 4.10: Total tropospheric $\mathrm{NO}_{2} \mathrm{AMF}$ as a function of cloud fraction in the (a) backward scattering direction and (b) forward scattering direction computed with surface BRDF (green) and a Lambertian surface (blue), for $\mathrm{P}_{\mathrm{cd}}=850 \mathrm{hPa},\left(\theta, \theta_{0}\right)=\left(45^{\circ}, 30^{\circ}\right)$, for a moderately polluted (stars) and unpolluted (circles) troposphere. BRDF parameters are $\left(f_{\text {iso }}, f_{\text {vol }}, f_{\text {geo }}\right)$ $=(0.04,0.03,0.008)$ and $\mathrm{A}_{\mathrm{ws}}=0.036$ for the Lambertian surface.

high bias in retrieved cloud pressure implies that the results shown for $850 \mathrm{hPa}$ might be representative of the surface BRDF effects on AMFs for clouds currently retrieved at higher (biased) pressures.

\subsubsection{GOME-2A tropospheric $\mathrm{NO}_{2}$ air mass factors}

We calculate Lambertian and $\mathrm{BRDF} \mathrm{NO}_{2}$ tropospheric AMFs for the exact illumination and viewing geometries of GOME-2A measurements over Amazonia and over France for March 2008. This results in approximately 1300 clear-sky pixels analyzed over Amazonia and 700 over France. We assume a moderately polluted atmosphere in every scene and collocate MODIS pixels with the GOME-2A pixel centre to obtain the surface BRDF parameters. For the Lambertian simulations, we use the surface LER value of each pixel from the GOME-2 climatology. We apply Lambertian and BRDF $c_{\text {eff }}$ distributions from Sect. 4.4.2 (as in Fig. 4.8). This way we account for the calculated surface BRDF effects in cloud fraction instead of the average change of 0.05 assumed in the sensitivity analysis in Sect. 4.5.1.

Figure 4.12a shows that for East measurements (i.e., forward scattering), $M_{\mathrm{cr}}$ decreases 

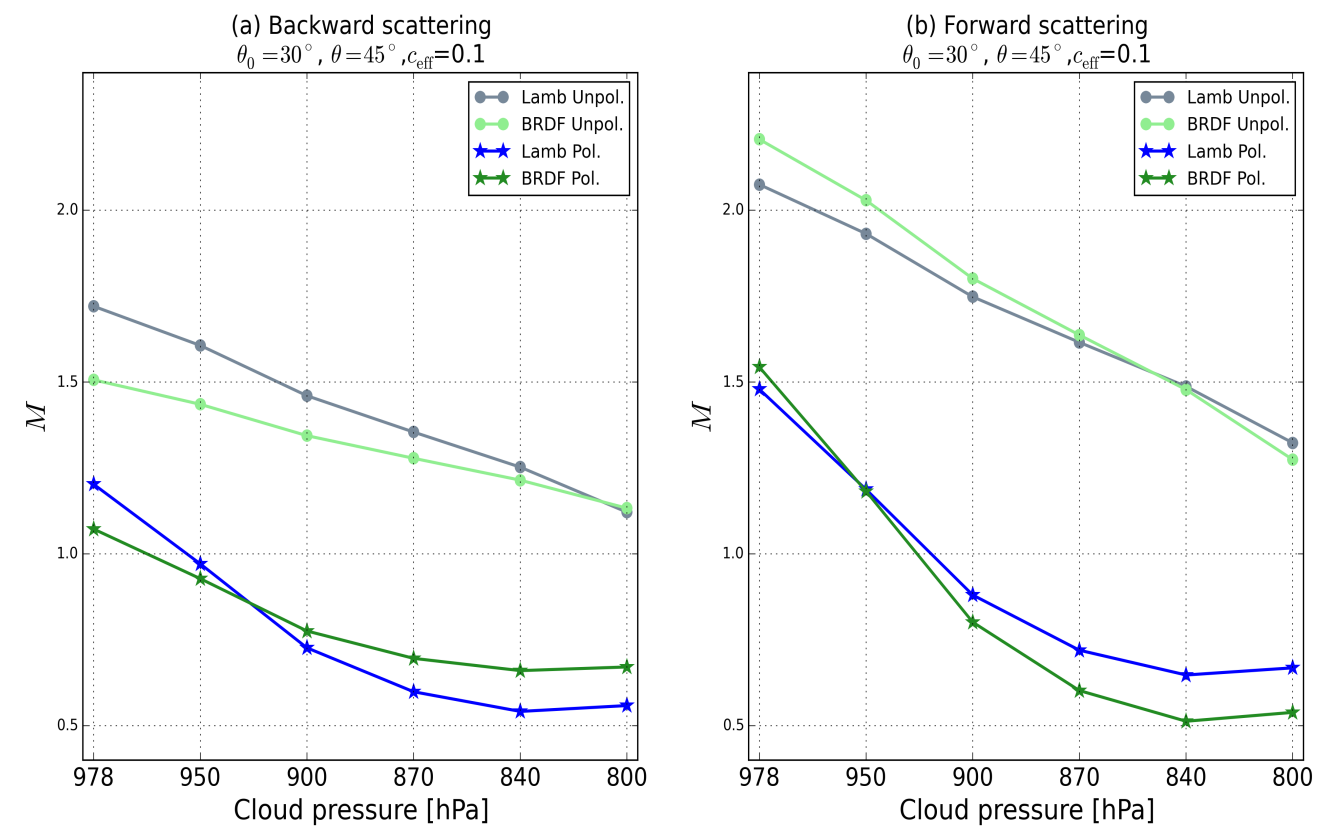

Figure 4.11: Total tropospheric $\mathrm{NO}_{2} \mathrm{AMF}$ as a function of cloud fraction in the (a) backward scattering direction and (b) forward scattering direction computed with surface BRDF (green) and a Lambertian surface (blue), for $\mathrm{P}_{\mathrm{cd}}=850 \mathrm{hPa},\left(\theta, \theta_{0}\right)=\left(45^{\circ}, 30^{\circ}\right)$, for a moderately polluted (stars) and unpolluted (circles) troposphere. BRDF parameters are $\left(f_{\text {iso }}, f_{\text {vol }}, f_{\text {geo }}\right)$ $=(0.04,0.03,0.008)$ and $\mathrm{A}_{\mathrm{ws}}=0.036$ for the Lambertian surface.

on average by $18 \%$ over Amazonia. Over France the decrease is on average $8 \%$ (not shown). Figure $4.12 \mathrm{~b}$ shows that for West measurements (i.e., backward scattering), $M_{\mathrm{cr}}$ increases on average 5\% over Amazonia and 7\% over France, consistent with our findings in Fig. 4.9a. The differences of 15\%-23\% found in this analysis agree with the reported differences of 10\%-20\% in Noguchi et al. (2014) and Zhou et al. (2010) for clear-sky AMFs. The higher BRDF AMFs in the upper right corner of our study area correspond to a savanna ecosystem that is brighter than the dense rainforest. Because of the higher resolution of the MODIS BRDF dataset, this feature is well captured and leads to higher AMFs both in the East and West measurements (see Fig. S4.2 in the supplementary material). MODIS albedo is able to capture spatial variability at scales that satellites with coarser pixels cannot (Russell et al., 2011).

Figure 4.12c shows that for East measurements (i.e., forward scattering), total tropospheric AMFs including surface BRDF effects on cloud fractions are on average $10 \%$ lower over Amazonia. The decrease is on average 7\% over France (not shown). For West measurements, (i.e., backward scattering), total AMFs are on average $16 \%$ higher over both Amazonia (Fig. 4.12d) and France, illustrating that the $M_{\text {cr }}$ effect is enhanced by the effect on the cloud fraction by $10 \%-15 \%$ on average. Vasilkov et al. (2017) also found increased differences in the tropospheric AMFs due to surface BRDF effects on 

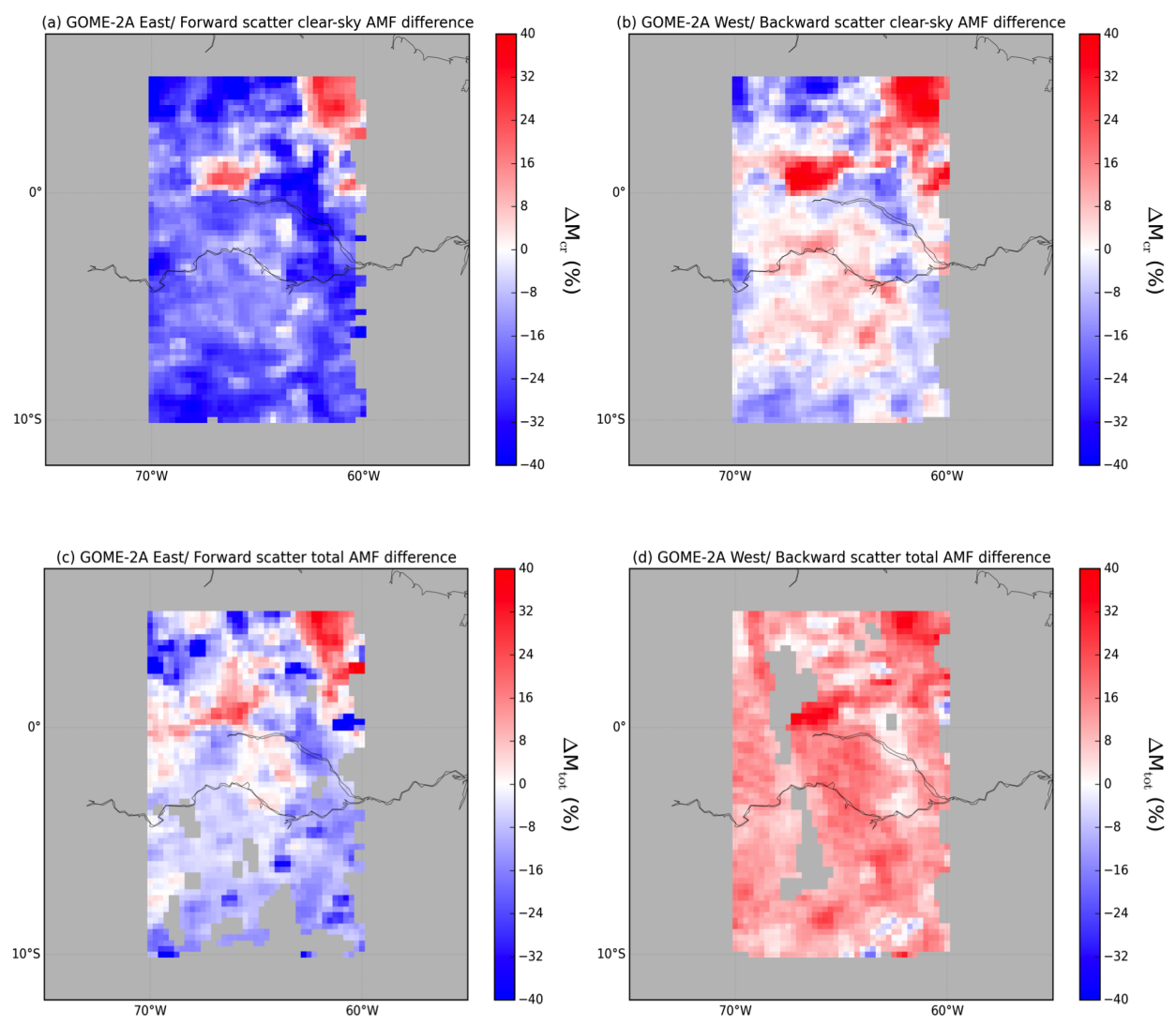

Figure 4.12: Relative differences between BRDF and Lambertian AMFs over Amazonia for March 2008: (a) clear-sky tropospheric $\mathrm{NO}_{2}$ AMFs for East GOME-2A measurements (forward scatter) (b) clear-sky tropospheric $\mathrm{NO}_{2}$ AMFs for West GOME-2A measurements (backward scatter) (c) total tropospheric $\mathrm{NO}_{2}$ AMF for East GOME-2A measurements (forward scatter) and (d) total tropospheric $\mathrm{NO}_{2}$ AMF for West GOME-2A measurements (backward scatter). Total AMFs are only shown for $\mathrm{c}_{\text {eff }}<0.5$.

cloud paramertes, but as reported by Lin et al. (2014), there can also be compensating effects.

These results show that surface BRDF affects both clear-sky AMF and cloud radiance fractions, which in combination significantly affect total $\mathrm{NO}_{2}$ AMFs. As shown in Fig. $4.10-4.12$, the sign and magnitude of the surface BRDF effects show strong spatial variations and depend on cloud fraction and cloud pressure. In order to generalize the effects to a global retrieval, a full assessment including all possible retrieval conditions should be done. Over forested terrain, current tropospheric AMF are likely underestimated in the backscatter regime and overestimated in the forward scattering regime by up to 25-35\%, explained by systematic errors in $M_{\mathrm{cr}}$ and $w$. These results show that surface BRDF effects have to be included consistently in both cloud and trace gas retrievals. 


\subsection{Discussion and conclusions}

We analysed the effects of surface reflectance anisotropy on the OMI and GOME-2 satellite retrievals of cloud parameters and tropospheric $\mathrm{NO}_{2}$ columns that currently use Lambertian-equivalent reflectivity (LER) climatologies. These climatologies, and consequently retrieved cloud fractions, show substantial across-track biases over terrain with a strong BRDF directionality. Here we interpret these with the DAK radiative transfer model. A clear understanding of the reasons for the biases and how they propagate in the tropospheric $\mathrm{NO}_{2}$ column retrieval is critical to improve cloud and trace gas retrieval algorithms for satellite sensors.

An important finding is that the LER climatologies slightly overestimate surface albedo for forward scattering satellite viewing geometries (eastern part of GOME-2 orbit), and highly underestimate the surface albedo for backscatter viewing geometries (western part). The underestimation is as large as a factor of 2 over forested scenes in the near-infrared $(772 \mathrm{~nm})$. They are weaker but still relevant in the visible $(494 \mathrm{~nm})$, where surfaces are darker and Rayleigh scattering effects are stronger. Such across-track bias in surface LER propagates into the cloud fraction retrievals: we find biases in cloud fractions of up to $50 \%$ between backscatter and forward scattering geometries in the GOME-2 FRESCO and $26 \%$ in the OMI OMCLDO2 cloud algorithms. Time-of-day does not drive importance of surface BRDF effects, but specific viewing geometry and spectral range do.

To interpret the above biases, we extended the description of surface reflectance in DAK to include the geometrical surface reflecting properties via the Bidirectional Reflectance Distribution Function (BRDF) from the Ross-Li semi-empirical model. This allows DAK to simulate not only isotropic reflection at the surface, but also the anisotropic contributions from volumetric (e.g. leaf scattering) and from geometric (e.g. shadowcasting) effects. We evaluated DAK top-of-atmosphere (TOA) reflectance simulations against other radiative transfer models, and find agreement within $1 \%$ between DAK and SCIATRAN, even within the so-called "hot-spot" backscatter reflectance peak. We then simulated TOA reflectances over vegetated scenes using BRDF parameters from a daily, high-resolution database derived from 16-years of MODIS measurements recently developed within the QA4ECV-project (QA4ECV-WP4, 2016). Our updated DAK simulations show considerably higher TOA reflectance levels for backscatter viewing geometries than those with isotropic surface reflection (LER) only. This strongly indicates that acrosstrack biases in cloud fractions can be explained by the lack of a description of surface reflectance anisotropy in the FRESCO and OMCLDO2 algorithms.

Subsequent sensitivity tests indicated that accounting for surface reflectance anisotropy in the FRESCO and OMCLDO2 retrieval framework removes the bias in cloud fractions. A correct physical description of surface anisotropy is essential for FRESCO, 

retrievals from GOME-2 and OMI

because cloud properties are retrieved in the NIR spectral range $(760-790 \mathrm{~nm})$ where surface BRDF effects are stronger and the atmosphere is virtually transparent. It is also of high relevance for scenes with low cloud fractions, where trace gas retrievals are still sensitive to pollution close to the ground. A discussion on the validity of the Lambertian cloud model is beyond the scope of this study. Nevertheless, the cloud fraction dependency with VZA for cloudy scenes suggests that the use of a more realistic cloud model should be considered in future improvements of cloud retrievals.

The implications for $\mathrm{NO}_{2}$ air mass factor (AMF) calculations are substantial. Total tropospheric $\mathrm{NO}_{2}$ AMFs are calculated as the radiative cloud fraction-weighted sum of cloudy and clear-sky AMFs. For moderately polluted $\mathrm{NO}_{2}$ and backscatter geometries, we find that clear-sky AMFs are up to $20 \%$ higher and cloud radiance fractions up to $40 \%$ lower if surface reflectance anisotropic effects are accounted for. The combined effect of these changes (with clouds located at $850 \mathrm{hPa}$ ) is that $\mathrm{NO}_{2}$ AMFs in polluted situations increase by $25-30 \%$ for backscatter geometries (and decrease by $25-35 \%$ for forward scattering geometries), stronger than the effect of either contribution alone.

An issue that was not addressed in this study is the role of aerosols. Noguchi et al. (2014) showed that scattering by aerosols generally dampens surface BRDF effects for clear-sky scenes. However, more research is needed to assess how specific aerosol characteristics (i.e. aerosol amount and type, vertical distribution relative to cloud) will affect cloud parameter retrievals and air mass factor calculations both in clear-sky and cloudy conditions.

We conclude that it is necessary to coherently account for surface reflectance anisotropy effects in retrievals of cloud properties and trace gases from UV/vis satellite sensors. Although this study does not apply surface BRDF to a complete global cloud and $\mathrm{NO}_{2}$ retrieval, it shows that it has substantial effects both cloud fractions and $\mathrm{NO}_{2}$ AMFs. A number of recent studies have attempted to account for the effects of anisotropic reflectance on both cloud and $\mathrm{NO}_{2}$ retrievals (Lin et al. (2014); Vasilkov et al. (2017)), but a global assessment including the full range of possible retrieval conditions is still missing. An additional incentive to account for surface reflectance anisotropy is that the currently available LER climatologies (Kleipool et al. (2008); Tilstra et al. (2017)) describe the spatial variation in albedo at a scale $\left(0.5^{\circ} \times 0.5^{\circ}-1^{\circ} \times 1^{\circ}\right)$ coarser than the OMI or GOME-2 pixel itself $\left(13 \times 24 \mathrm{~km}^{2} / 80 \times 40 \mathrm{~km}^{2}\right)$. Using these coarse LER climatologies in AMF calculations degrades the intrinsic spatial resolution of the satellite retrievals, an issue that will be exacerbated for the recently launched TROPOMI instrument, with pixels as small as $3.5 \times 7 \mathrm{~km}^{2}$. A viable alternative to the current LER climatologies is provided by the MODIS-derived BRDF-parameters at a spatial resolution better than the GOME-2, OMI, and TROPOMI pixel sizes. MODIS Terra and Aqua are expected to last until 2025 and afterwards the Joint Polar Satellite System (JPSS) satellite constellation assures con- 
tinuity of land observations needed to produce surface BRDF data. Sentinel-3 could be employed to generate a BRDF similar to the one from the ESA GlobAlbedo broadband and the QA4ECV spectral albedo after some years of measurements. Another alternative is to make a directionally dependent LER database from TROPOMI once there is enough surface reflectance data acquired by the satellite itself.

\section{Acknowledgements}

This research has been supported by the EU FP7 Project Quality Assurance for Essential Climate Variables (QA4ECV), grant no. 607405. We acknowledge the free use of the GOME-2 data provided by EUMETSAT. 



\title{
5 Quantification of nitrogen oxides emissions from build-up of pollu- tion over Paris with TROPOMI
}

\begin{abstract}
Nitrogen dioxide $\left(\mathrm{NO}_{2}\right)$ is a regulated air pollutant that is of particular concern in many cities, where concentrations are high. Emissions of nitrogen oxides to the atmosphere lead to the formation of ozone and particulate matter, with adverse impacts on human health and ecosystems. The effects of emissions are often assessed through modelling based on inventories relying on indirect information, that is often outdated or incomplete. Here we show that $\mathrm{NO}_{2}$ measurements from the new, high-resolution TROPOMI satellite sensor can directly determine the strength and distribution of emissions from Paris. From the observed build-up of $\mathrm{NO}_{2}$ pollution, we find highest emissions on cold weekdays in February 2018, and lowest emissions on warm weekend days in spring. The new measurements provide information on the spatio-temporal distribution of emissions within a large city, and suggest that Paris emissions in 2018 are only 5-15\% below inventory estimates for 2011-2012, reflecting the difficulty of meeting $N O_{x}$ emission reduction targets.
\end{abstract}

A modified version of this chapter is under review for Scientific Reports as:

Lorente, A., Boersma, K. F., Eskes, H. J., Veefkind, J. P., van Geffen, J. H. G. M. , de Zeeuw, J. H. G. M., Denier van der Gon, H., Beirle, S. and Krol, M.C.: Quantification of nitrogen oxides emissions from build-up of pollution over Paris with TROPOMI (25-01-2019). 


\subsection{Introduction}

Nitrogen oxides, mostly a product of combustion processes, play a key role in tropospheric chemistry, and influence air quality and atmospheric radiative forcing (Myhre et al., 2013). Nitrogen oxides are short-lived (1-12 hours) (Stavrakou et al., 2013), but their photochemical processing leads to longer lasting effects via the formation of ozone (Stevenson et al., 2006) and aerosols, as well as acid rain (Jacob, 1999). In response, European Union legislation establishes a maximum acceptable nitrogen dioxide $\left(\mathrm{NO}_{2}\right)$ concentration in ambient air of $40 \mu \mathrm{g} / \mathrm{m}^{3}$. In 2016, this annual limit for $\mathrm{NO}_{2}$ was widely exceeded across Europe (EEA, 2018). For example, 1.4 million Parisians were exposed to $\mathrm{NO}_{2}$ levels exceeding the limit in that year, mostly because of strong emissions from road traffic, and from residential and commercial combustion (Airparif, 2017), despite sizeable reductions in emissions reported over the last decade (EEA, 2017). Reliable and comprehensive emission estimates are needed to evaluate air quality mitigation strategies and as input to models simulating and forecasting air pollution. Satellite measurements provide a comprehensive perspective on the spatial distribution (e.g. Miyazaki et al. (2017), Fioletov et al. (2013)) and temporal evolution (e.g. Richter et al. (2005), Lamsal et al. (2011)) of global emissions. Such emission estimates are still limited in their spatial and temporal resolution. Thus, there remains a need to better understand emissions, in particular their driving factors, in order to provide accurate alternatives for bottom-up estimates at the sub-urban scale and on a day-to-day basis.

Here we report on $\mathrm{NO}_{x}$ emission estimates from new $\mathrm{NO}_{2}$ measurements by TROPOMI (Veefkind et al., 2012) instrument on the Sentinel-5 Preparatory (S5P) mission. TROPOMI extends the data records obtained from SCIAMACHY (2002-2012), GOME-2 (ongoing since 2007), and OMI (since 2004) sensors, and is the preparatory mission for Sentinel-5, due for launch in the 2020s. TROPOMI is a spectrometer measuring direct and reflected sunlight at around 13:30 hrs local time in ultraviolet and visible bands, as well as radiance and irradiance in the near- and short-wave infrared. Besides $\mathrm{NO}_{2}$, these spectral bands allow the observation of other atmospheric constituents such as ozone, carbon monoxide (Borsdorff et al., 2018), sulfur dioxide, formaldehyde (De Smedt et al., 2018), and methane (Hu et al., 2018), as well as aerosol and cloud properties. Satellite data quality has gradually increased over the last decade, but the very high spatial resolution of $3.5 \times 7 \mathrm{~km}^{2}$, (across $\times$ along track) and improved signal-to-noise offered by TROPOMI (van Geffen et al., 2018) promise to be a major step forward.

The tropospheric $\mathrm{NO}_{2}$ data from TROPOMI (van Geffen et al. (2018), Boersma et al. (2018)) provide the opportunity to estimate the $\mathrm{NO}_{x}$ emissions directly from the buildup of $\mathrm{NO}_{2}$ observed over Paris on a single clear-sky days, without the need for complex inversions with a chemistry-transport model (Beirle et al., 2011). Our method allows 
inferring the $\mathrm{NO}_{x}$ emissions from the observed $\mathrm{NO}_{2}$ in air advected over the city, provided that wind speed and wind direction are known to good accuracy. Under non-stagnant conditions, the chemical decay of $\mathrm{NO}_{2}$ in the boundary layer is of minor importance given the short time it takes for an air parcel to cross the Paris Metropolitan area relative to the chemical lifetime (Stavrakou et al. (2013), Shaiganfar et al. (2017), Beirle et al. (2011)). The quality of the $\mathrm{NO}_{2}$ retrievals from TROPOMI is such, that it is not necessary to reduce noise by averaging satellite $\mathrm{NO}_{2}$ distributions for a particular wind direction sector first (van Geffen et al. (2018), Beirle et al. (2011)). This avoids errors associated with interpreting average patterns based on an ensemble of individual plumes from different days with different wind directions and wind speeds. Instead, we directly analyse the build-up of $\mathrm{NO}_{2}$ pollution over the city from an individual TROPOMI orbit, thereby achieving one or sometimes even two direct estimates of city-wide $\mathrm{NO}_{x}$ emissions on a particular day.

Figure 5.1a and 5.1b illustrate that the TROPOMI measurements present an improvement over OMI, with TROPOMI clearly capturing the details of the $\mathrm{NO}_{2}$ pollution plume originating from Paris and blown to the north on 17 April 2018. Indeed, Fig. 5.1c shows that wind direction and speed from the European Centre for Medium Range Weather Forecasts (Dee et al., 2011) indicate a southerly flow on this day, which was mostly cloudfree at the overpass (Fig. 5.1d). The tropospheric $\mathrm{NO}_{2}$ columns from TROPOMI are per definition representative for the vertically integrated $\mathrm{NO}_{2}$ concentrations between the surface and the tropopause, and they are directly linked to the total $\mathrm{NO}_{x}$ emissions. We select measurements taken under mostly cloud-free conditions (see Sect. 5.4), when TROPOMI is having good sensitivity to enhanced $\mathrm{NO}_{2}$ concentrations in the polluted boundary layer.

\section{$5.2 \quad$ Results}

\subsection{1 $\quad \mathrm{NO}_{2}$ build-up in air advected over Paris}

Paris is one of the three megacities in Europe, next to London and Istanbul, and one of the strongest isolated hotspots of air pollution in north-western Europe, with 10.5 million inhabitants and more than 3 million cars entering the city each day. Figure 5.2 shows tropospheric $\mathrm{NO}_{2}$ columns over this region on Friday 23 February 2018 measured by TROPOMI. The spatial distribution shows an increase in $\mathrm{NO}_{2}$ columns from the northeast towards the south-west over Paris, and downwind of the city a plume of enhanced $\mathrm{NO}_{2}$ advected towards the south-west, consistent with predominantly north-easterly winds $(32 \mathrm{~km} / \mathrm{h})$ on that day. $\mathrm{NO}_{2}$ surface concentrations measured at 20 stations throughout Paris (Airparif, 2018) suggest a similar increase in surface pollution from the north-east 

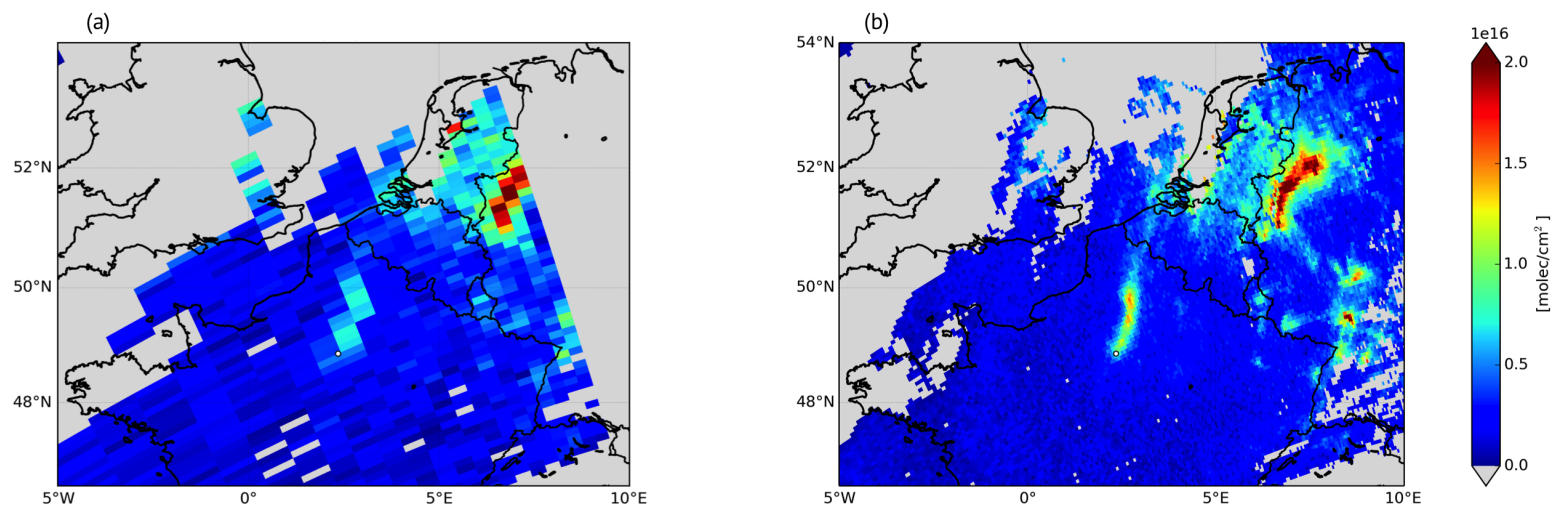

(c)
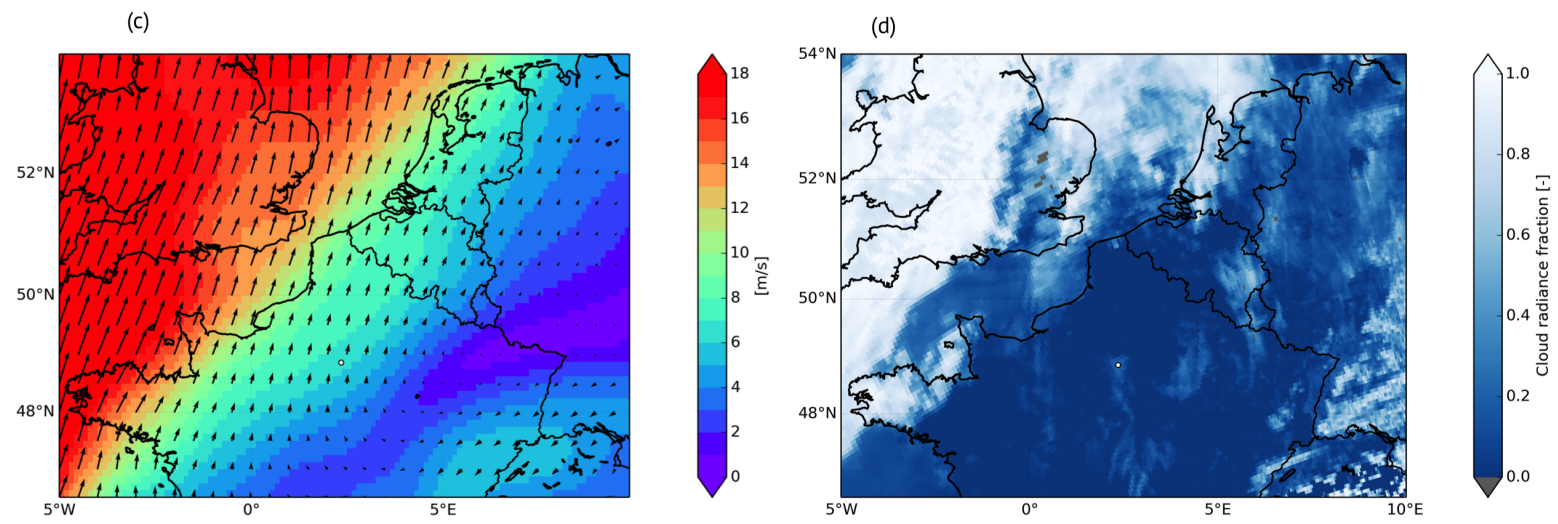

Figure 5.1: Tropospheric $\mathrm{NO}_{2}$ vertical column on 17 April 2018 from (a) OMI (DOMINO v2), and (b) TROPOMI. (c) ECMWF Era Interim 10 meter wind speed on 17 April 2018 at 12:00 UTC, (d) TROPOMI cloud radiance fraction. In (a-b) data has been filtered for cloud radiance fraction (lower than 0.5) and surface albedo (lower than 0.3), and grey areas indicate cloudiness or no data (in the case of OMI). Measurement time was approximately 12:15 UTC for both OMI and TROPOMI. Paris is indicated as a small white circle in the panels.

towards the south-west, even though the measurement techniques are very different. Two days later, on Sunday 25 February 2018, the wind $(40 \mathrm{~km} / \mathrm{h}$ ) was slightly stronger, but the build-up of $\mathrm{NO}_{2}$ over the city was much weaker, a first indication of lower emissions on this weekend day. This analysis for two different days suggests that direct attribution of the $\mathrm{NO}_{2}$ increase over Paris to the $\mathrm{NO}_{x}$ source strength is possible, if the influence of wind speed and $\mathrm{NO}_{x}$ loss processes are accounted for.

On days with very low wind speeds, $\mathrm{NO}_{x}$ emissions from the city are hardly ventilated, but concentrate over the city instead. On such days, the satellite $\mathrm{NO}_{2}$ distribution over Paris closely resembles the underlying emission pattern. Figure 5.2e shows the $\mathrm{NO}_{2}$ column distribution on Wednesday 20 June 2018, a day with a high-pressure system centred over Paris. In the hours prior to TROPOMI overpass, a very light wind (0-2 $\mathrm{km} / \mathrm{hr}$ ) was coming from the north (Venstusky, 2018). The high $\mathrm{NO}_{2}$ columns observed over inner Paris display a clear echo of the underlying spatial distribution of $\mathrm{NO}_{x}$ emissions 
(Figure S5.2). The $\mathrm{NO}_{2}$ distributions on 20 June 2018 can be used to estimate the $\mathrm{NO}_{x}$ emissions, but the photochemical regime is more complex in stagnant conditions, and assumptions on the chemical lifetime of $\mathrm{NO}_{x}$ are more critical than in well-ventilated situations, when $\mathrm{NO}_{2}$ loss over the city is dominated by transport of polluted air out of the city (e.g. Jacob (1999), Shaiganfar et al. (2017). From a simple scaling argument this becomes evident: with a wind speed of $30 \mathrm{~km} / \mathrm{h}$, it takes 2 hours for an air parcel to cross the city, too short for substantial chemical loss of $\mathrm{NO}_{x}$. With a wind speed of $5 \mathrm{~km} / \mathrm{h}$ however, it takes 12 hours, long enough for substantial chemical conversion of $\mathrm{NO}_{2}$ into $\mathrm{HNO}_{3}$ over the city.

\subsubsection{Interpreting line densities}

By integrating the TROPOMI $\mathrm{NO}_{2}$ columns perpendicular to the wind direction over a distance of $60 \mathrm{~km}$, we constructed the so-called line densities (Beirle et al., 2011) (see Sect. 5.4). Increases in $\mathrm{NO}_{2}$ line density along with the wind are directly linked to recent $\mathrm{NO}_{x}$ emissions over the metropolitan area, somewhat dampened by photochemical decay. The right panels of Fig. 5.2 show the $\mathrm{NO}_{2}$ line densities for 23 and 25 February 2018, and for 20 June 2018 as function of the along-wind distance over Paris. For the windy February days, the line densities show a modest increase of $\mathrm{NO}_{2}$ with distance over the north-eastern segment of the city, followed by a steeper increase over and just downwind of the city centre, and a levelling off towards the south-western edge of the city. This pattern is apparent on many days (Fig. S5.7) and reflects the spatial distribution of emissions within the Paris Metropolitan Area, where most $\mathrm{NO}_{x}$ is emitted in the region enveloped by the A86 ring road, and much weaker emissions occur in the outskirts (Fig. S5.2). On days with high wind speeds, line densities over the city provide a convolved view of the underlying emission pattern, because the wind takes along and disperses the recently emitted nitrogen oxides. On days when wind speeds were extremely low, such as 20 June, line densities closely resemble the spatial distribution of the $\mathrm{NO}_{x}$ emissions at the approximate scale of a TROPOMI pixel.

\subsubsection{Estimating $\mathrm{NO}_{x}$ emissions from TROPOMI line densities}

We validated the TROPOMI $\mathrm{NO}_{2}$ columns over Paris. We compared the TROPOMI columns against a set of coincident $\mathrm{NO}_{2}$ columns inferred from in situ (AirParif) $\mathrm{NO}_{2}$ measurements taken on the Eiffel Tower using information on boundary layer height (Dieudonné et al., 2013) (see Sect. S5.1). The comparison suggests excellent agreement $\left(\mathrm{R}^{2}=0.88\right)$ between the TROPOMI and AirParif columns, but the variability in TROPOMI $\mathrm{NO}_{2}$ columns is underestimated by $25 \%$ (Figure S5.1). This bias, most likely caused by air mass factor errors, would lead to a similar low bias in the $\mathrm{NO}_{x}$ emission 
(a)
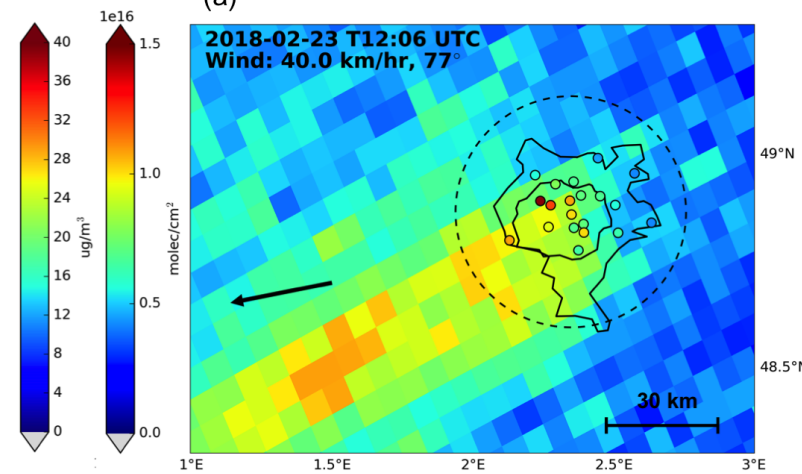

(c)
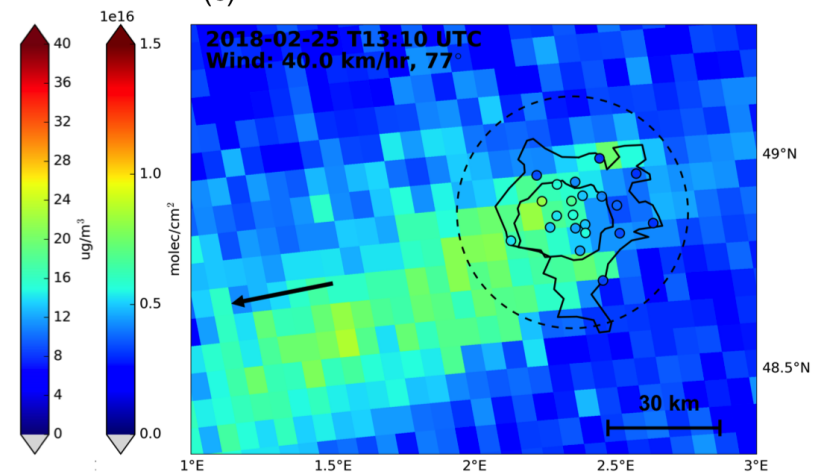

(e)
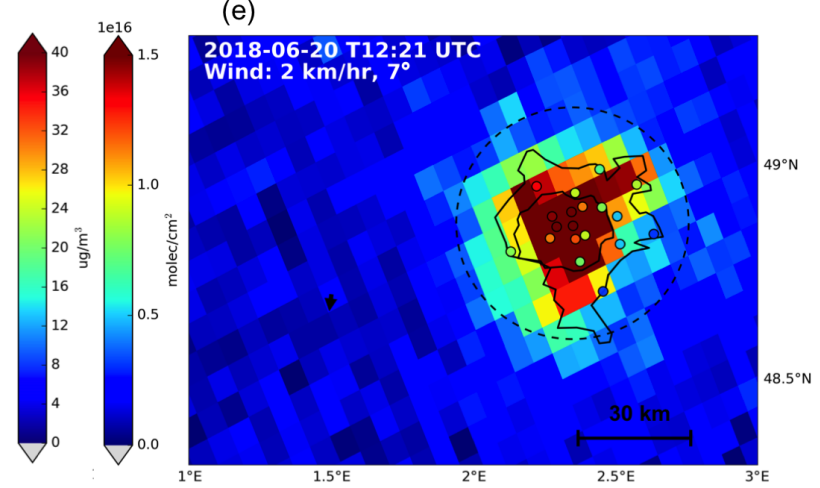

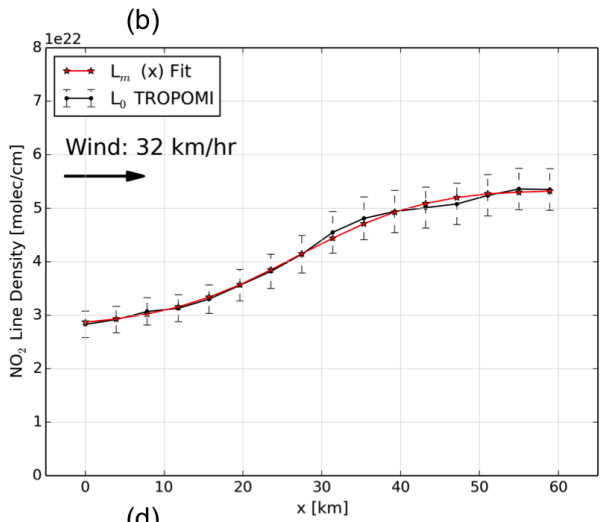

(d)
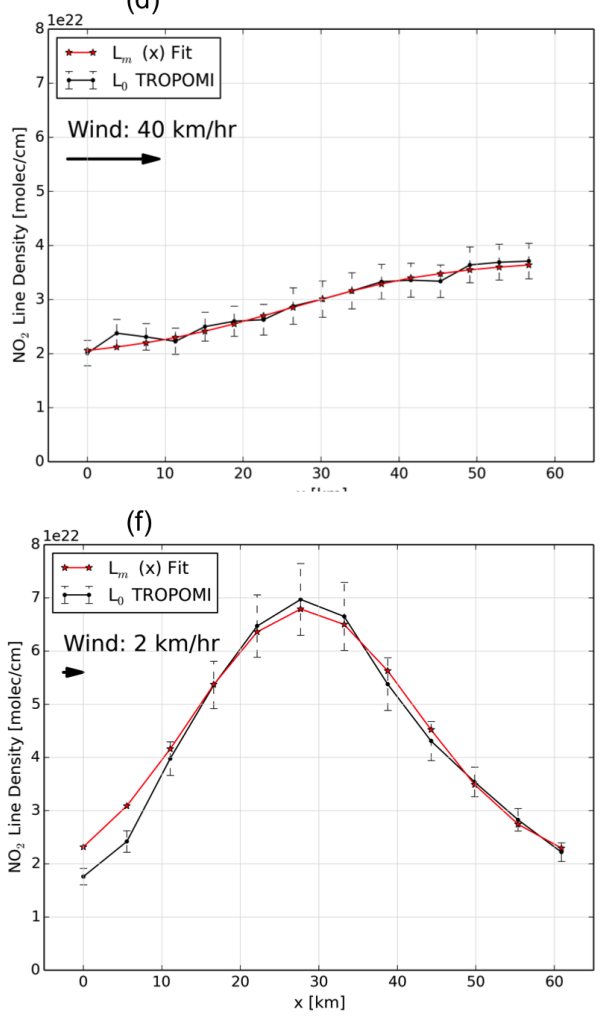

Figure 5.2: (a) Tropospheric $\mathrm{NO}_{2}$ columns over Paris on Friday 23 February 2018, (c) Sunday 25 February 2018, and (e) Wednesday 20 June 2018 observed by TROPOMI. Boundary layer mean wind speed and wind direction, indicated by the black arrow, are from ECMWF ERAInterim data. The Paris A86 ring road is indicated by the inner black line, and the city limits are approximated by the outer black line. The right panels (b), (d), and (f) show the corresponding $\mathrm{NO}_{2}$ line densities between the upwind and downwind city limits at 0 and 60 $\mathrm{km}$.

estimates, so we correct for this bias when estimating $\mathrm{NO}_{x}$ emissions from the line densities.

To determine $\mathrm{NO}_{x}$ emissions from the observed $\mathrm{NO}_{2}$ build-up on clear-sky days in the first half of 2018, we generate a large ensemble of pre-computed line densities, each a 
of strength and pattern of the $\mathrm{NO}_{x}$ emissions, wind speed, and $\mathrm{NO}_{x}$ loss rate constant (see Sect. 5.4). We compared each member to the observed line density, to identify the function and its driving parameters that minimized the differences between the modelled and the observed line density. In the ensemble, we take as prescribed parameters the boundary layer average wind speed from ECMWF, and the $\left[\mathrm{NO}_{2}\right]:\left[\mathrm{NO}_{x}\right]$ ratios simulated by the CAMS model (Inness et al., 2015) over Paris for the day of interest (see Sect. 5.4). We allow the $\mathrm{NO}_{x}$ loss rate constants and the emission pattern (with 12 cells of $5 \mathrm{~km}$ over Paris) to vary within a predefined range in our ensemble. As a first guess of the $\mathrm{NO}_{x}$ loss rate constant, we use boundary layer mean $[\mathrm{OH}]$ from CAMS with a range of $\pm 50 \%$ given the considerable uncertainty in simulating OH over a large city (Shaiganfar et al. (2017), Ren et al. (2003), Valin et al. (2013)) by models (see Fig. S5.5). The emission pattern is inherently uncertain, so we also allow this to vary. As a first guess pattern we use the 1-D

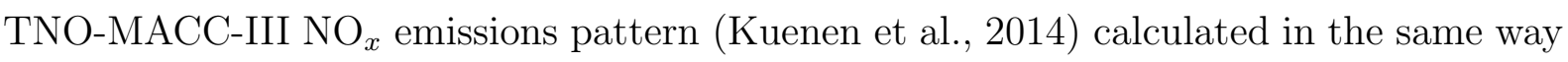
as we calculated the $\mathrm{NO}_{2}$ line densities, which resembles a Gaussian distribution (Fig. S5.6). We then use in our ensemble a range of Gaussian shapes defined by variation of 4 parameters: amplitude (up to factor 3 different), widths $( \pm 15 \%)$, offsets (factor 2 ), and centre $( \pm 5 \mathrm{~km})$ locations. The observed along-wind line densities are described well by the modelled function giving the smallest residuals, as illustrated in the right panels of Fig. 5.2, and by the high correlation coefficients between the observations and modelled function (average $\mathrm{R}^{2}=0.953$ ), and low unexplained residuals (approximately $13 \%$ of the line density values). Because of the TROPOMI overpass of approximately 13:40 hrs local time, the inferred $\mathrm{NO}_{x}$ emissions are generally representative for the hours just prior to the TROPOMI overpass, i.e. noontime (Valin et al., 2013). Table 5.1 summarizes our main results.

Table 5.1: Paris Metropolitan Area $\mathrm{NO}_{x}$ emissions inferred from TROPOMI measurements in 2018, and the conditions under which they have been derived. Days with two estimates Paris was covered by two successive orbits (orbit 1916 and 1917 on 25 February, 2895 and 2896 on 5 May, 3548 and 3549 on 20 June, 3633 and 3634 on 26 June 2018).

\begin{tabular}{|c|c|c|c|c|c|c|c|c|c|}
\hline Day & $\begin{array}{c}\text { Time } \\
\text { (UTC) }\end{array}$ & $\begin{array}{l}\text { Emissions } \\
\mathrm{mol} \mathrm{s}^{-1}\end{array}$ & $\begin{array}{c}\text { Wind } \\
\text { Speed, Dir. }\end{array}$ & $\begin{array}{l}\text { NO }_{x} \\
\text { lifetime }\end{array}$ & $\begin{array}{c}{\left[\mathrm{NO}_{x}\right]:\left[\mathrm{NO}_{2}\right]} \\
\text { CAMS, } \\
\text { Eiffel Tower }\end{array}$ & $\begin{array}{l}\text { Surface } \\
\text { temp. }\end{array}$ & $\begin{array}{c}\text { PBL } \\
\text { height } \\
(\mathrm{m})\end{array}$ & $\mathbf{R}^{2}$ & $\begin{array}{l}\text { RMS } \\
\text { res. }\end{array}$ \\
\hline $\begin{array}{c}\text { Thu } \\
22.02\end{array}$ & $12: 25$ & $110.0 \pm 37.4$ & $8.6 \mathrm{~m} / \mathrm{s}, 51^{\circ}(\mathrm{NE})$ & $16.0 \mathrm{hrs}$ & $1.83,1.86$ & $3^{\circ} \mathrm{C}$ & 874 & 0.990 & $8 \%$ \\
\hline $\begin{array}{c}\text { Fri } \\
23.02\end{array}$ & $12: 06$ & $93.3 \pm 31.3$ & $9.0 \mathrm{~m} / \mathrm{s}, 70^{\circ}(\mathrm{ENE})$ & $10.9 \mathrm{hrs}$ & $1.72,1.70$ & $2^{\circ} \mathrm{C}$ & 907 & 0.996 & $4 \%$ \\
\hline $\begin{array}{c}\text { Sun } \\
25.02\end{array}$ & $11: 29$ & $51.3 \pm 16.5$ & $11.0 \mathrm{~m} / \mathrm{s}, 77^{\circ}(\mathrm{E})$ & $9.4 \mathrm{hrs}$ & $1.75,1.78$ & $0^{\circ} \mathrm{C}$ & 798 & 0.933 & $15 \%$ \\
\hline $\begin{array}{c}\text { Sun } \\
25.02\end{array}$ & $13: 10$ & $74.4 \pm 23.9$ & $11.0 \mathrm{~m} / \mathrm{s}, 77^{\circ}(\mathrm{E})$ & $11.4 \mathrm{hrs}$ & $1.75,1.86$ & $0^{\circ} \mathrm{C}$ & 798 & 0.973 & $10 \%$ \\
\hline $\begin{array}{c}\text { Mon } \\
26.02\end{array}$ & $12: 51$ & $78.7 \pm 24.9$ & $12.2 \mathrm{~m} / \mathrm{s}, 56^{\circ}(\mathrm{NE})$ & $10.3 \mathrm{hrs}$ & $1.73,1.78$ & $-2^{\circ} \mathrm{C}$ & 1545 & 0.925 & $21 \%$ \\
\hline $\begin{array}{c}\text { Tue } \\
17.04\end{array}$ & $12: 18$ & $87.6 \pm 26.9$ & $6.4 \mathrm{~m} / \mathrm{s}, 197^{\circ}(\mathrm{S})$ & $1.6 \mathrm{hrs}$ & $1.39,1.58$ & $17^{\circ} \mathrm{C}$ & 1187 & 0.995 & $5 \%$ \\
\hline
\end{tabular}


Quantification of nitrogen oxides emissions

Table 5.1 - continued

\begin{tabular}{|c|c|c|c|c|c|c|c|c|c|}
\hline Day & $\begin{array}{c}\text { Time } \\
\text { (UTC) }\end{array}$ & $\begin{array}{l}\text { Emissions } \\
\mathrm{mol} \mathrm{s}^{-1}\end{array}$ & $\begin{array}{c}\text { Wind } \\
\text { Speed, Dir. }\end{array}$ & $\begin{array}{l}\text { NO }_{x} \\
\text { lifetime }\end{array}$ & $\begin{array}{c}{\left[\mathrm{NO}_{x}\right]:\left[\mathrm{NO}_{2}\right]} \\
\text { CAMS, } \\
\text { Eiffel Tower }\end{array}$ & $\begin{array}{l}\text { Surface } \\
\text { temp. }\end{array}$ & $\begin{array}{c}\text { PBL } \\
\text { height } \\
(\mathrm{m})\end{array}$ & $\mathbf{R}^{2}$ & $\begin{array}{l}\text { RMS } \\
\text { resi. }\end{array}$ \\
\hline $\begin{array}{c}\text { Wed } \\
18.04 \\
\end{array}$ & $12: 59$ & $64.5 \pm 19.3$ & $6.9 \mathrm{~m} / \mathrm{s}, 100^{\circ}(\mathrm{E})$ & $2.0 \mathrm{hrs}$ & $1.19,1.16$ & $23^{\circ} \mathrm{C}$ & 1251 & 0.994 & $5 \%$ \\
\hline $\begin{array}{c}\text { Sat } \\
21.04\end{array}$ & $12: 44$ & $38.4 \pm 24.1$ & $2.7 \mathrm{~m} / \mathrm{s}, 100^{\circ}(\mathrm{E})$ & $2.9 \mathrm{hrs}$ & $1.31,1.28$ & $24^{\circ} \mathrm{C}$ & 2106 & 0.970 & $9 \%$ \\
\hline $\begin{array}{c}\text { Sun } \\
22.04\end{array}$ & $12: 25$ & $27.7 \pm 9.1$ & $9.7 \mathrm{~m} / \mathrm{s}, 230^{\circ}(\mathrm{SW})$ & $2.7 \mathrm{hrs}$ & $1.26,1.39$ & $24^{\circ} \mathrm{C}$ & 2188 & 0.840 & $31 \%$ \\
\hline \begin{tabular}{|c|} 
Tue \\
24.04 \\
\end{tabular} & $13: 29$ & $86.8 \pm 36.3$ & $5.0 \mathrm{~m} / \mathrm{s}, 231^{\circ}(\mathrm{SW})$ & $2.8 \mathrm{hrs}$ & 1.48,N.A. & $15^{\circ} \mathrm{C}$ & 1292 & 0.980 & $9 \%$ \\
\hline $\begin{array}{c}\text { Wed } \\
02.05 \\
\end{array}$ & $12: 39$ & $65.2 \pm 21.9$ & $9.0 \mathrm{~m} / \mathrm{s}, 206^{\circ}(\mathrm{SW})$ & $1.6 \mathrm{hrs}$ & $1.18,1.39$ & $14^{\circ} \mathrm{C}$ & 1499 & 0.990 & $7 \%$ \\
\hline $\begin{array}{c}\text { Fri } \\
04.05 \\
\end{array}$ & $12: 01$ & $50.4 \pm 18.6$ & $6.6 \mathrm{~m} / \mathrm{s}, 23^{\circ}(\mathrm{NNE})$ & $2.9 \mathrm{hrs}$ & $1.21,1.36$ & $16^{\circ} \mathrm{C}$ & 1051 & 0.957 & $12 \%$ \\
\hline $\begin{array}{c}\text { Sat } \\
05.05 \\
\end{array}$ & $11: 42$ & $49.2 \pm 18.0$ & $6.8 \mathrm{~m} / \mathrm{s}, 27^{\circ}(\mathrm{NNE})$ & $2.9 \mathrm{hrs}$ & $1.22,1.24$ & $19^{\circ} \mathrm{C}$ & 682 & 0.984 & $7 \%$ \\
\hline $\begin{array}{c}\text { Sat } \\
05.05\end{array}$ & $13: 23$ & $33.5 \pm 12.2$ & $6.8 \mathrm{~m} / \mathrm{s}, 27^{\circ}(\mathrm{NNE})$ & $1.8 \mathrm{hrs}$ & $1.22,1.11$ & $19^{\circ} \mathrm{C}$ & 682 & 0.964 & $10 \%$ \\
\hline $\begin{array}{c}\text { Sun } \\
06.05\end{array}$ & $13: 04$ & $24.8 \pm 10.1$ & $5.3 \mathrm{~m} / \mathrm{s}, 53^{\circ}(\mathrm{ENE})$ & $1.9 \mathrm{hrs}$ & $1.20,1.31$ & $23^{\circ} \mathrm{C}$ & 696 & 0.937 & $11 \%$ \\
\hline $\begin{array}{c}\text { Tue } \\
08.05 \\
\end{array}$ & $12: 26$ & $45.6 \pm 36.7$ & $2.0 \mathrm{~m} / \mathrm{s}, 5^{\circ}(\mathrm{N})$ & $2.1 \mathrm{hrs}$ & $1.23,1.16$ & $24^{\circ} \mathrm{C}$ & 1699 & 0.959 & $10 \%$ \\
\hline $\begin{array}{c}\text { Wed } \\
09.05 \\
\end{array}$ & $13: 49$ & $36.4 \pm 17.3$ & $4.0 \mathrm{~m} / \mathrm{s}, 300^{\circ}(\mathrm{WNW})$ & $2.5 \mathrm{hrs}$ & $1.16,1.17$ & $19^{\circ} \mathrm{C}$ & 775 & 0.990 & $7 \%$ \\
\hline $\begin{array}{c}\text { Fri } \\
11.05 \\
\end{array}$ & $13: 11$ & $48.4 \pm 20.9$ & $4.7 \mathrm{~m} / \mathrm{s}, 167^{\circ}(\mathrm{S})$ & $2.2 \mathrm{hrs}$ & $1.21,1.44$ & $17^{\circ} \mathrm{C}$ & 1491 & 0.982 & $9 \%$ \\
\hline $\begin{array}{c}\text { Thu } \\
17.05 \\
\end{array}$ & $12: 58$ & $44.8 \pm 18.7$ & $5.0 \mathrm{~m} / \mathrm{s}, 12^{\circ}(\mathrm{N})$ & $2.4 \mathrm{hrs}$ & $1.26,1.60$ & $15^{\circ} \mathrm{C}$ & 844 & 0.992 & $6 \%$ \\
\hline $\begin{array}{c}\text { Fri } \\
18.05 \\
\end{array}$ & $12: 39$ & $40.1 \pm 18.0$ & $4.4 \mathrm{~m} / \mathrm{s}, 21^{\circ}(\mathrm{N})$ & $3.2 \mathrm{hrs}$ & $1.24,1.72$ & $14^{\circ} \mathrm{C}$ & 787 & 0.983 & $9 \%$ \\
\hline $\begin{array}{c}\text { Sat } \\
19.05\end{array}$ & $12: 20$ & $36.4 \pm 17.3$ & $4.0 \mathrm{~m} / \mathrm{s}, 26^{\circ}(\mathrm{NNE})$ & $2.4 \mathrm{hrs}$ & $1.25,1.31$ & $18^{\circ} \mathrm{C}$ & 1038 & 0.903 & $24 \%$ \\
\hline $\begin{array}{c}\text { Wed } \\
20.06\end{array}$ & $12: 20$ & $54.3 \pm 57.3$ & $0.5 \mathrm{~m} / \mathrm{s}, 7^{\circ}(\mathrm{N})$ & $3.3 \mathrm{hrs}$ & $1.61,1.4$ & $25^{\circ} \mathrm{C}$ & 959 & 0.984 & $11 \%$ \\
\hline $\begin{array}{c}\text { Wed } \\
20.06 \\
\end{array}$ & $14: 00$ & $75.6 \pm 67.9$ & $1.8 \mathrm{~m} / \mathrm{s}, 7^{\circ}(\mathrm{N})$ & $3.9 \mathrm{hrs}$ & $1.55,1.4$ & $25^{\circ} \mathrm{C}$ & 959 & 0.942 & $20 \%$ \\
\hline $\begin{array}{c}\text { Thu } \\
21.06\end{array}$ & $12: 02$ & $30.5 \pm 12.8$ & $5.0 \mathrm{~m} / \mathrm{s}, 352^{\circ}(\mathrm{N})$ & $2.0 \mathrm{hrs}$ & $1.3,1.66$ & $20^{\circ} \mathrm{C}$ & 1139 & 0.947 & $15 \%$ \\
\hline $\begin{array}{c}\text { Sat } \\
23.06\end{array}$ & $13: 05$ & $17.7 \pm 8.3$ & $4.1 \mathrm{~m} / \mathrm{s}, 35^{\circ}(\mathrm{NNE})$ & $1.7 \mathrm{hrs}$ & $1.34,1.44$ & $19^{\circ} \mathrm{C}$ & 1094 & 0.653 & $38 \%$ \\
\hline \begin{tabular}{|c|} 
Mon \\
25.06 \\
\end{tabular} & $12: 27$ & $51.7 \pm 21.2$ & $5.2 \mathrm{~m} / \mathrm{s}, 46^{\circ}(\mathrm{NE})$ & $1.8 \mathrm{hrs}$ & $1.26,1.42$ & $23^{\circ} \mathrm{C}$ & 1591 & 0.976 & $11 \%$ \\
\hline $\begin{array}{c}\text { Tue } \\
26.06 \\
\end{array}$ & $12: 08$ & $44.8 \pm 22.8$ & $3.6 \mathrm{~m} / \mathrm{s}, 29^{\circ}(\mathrm{NNE})$ & $2.1 \mathrm{hrs}$ & $1.33,1.41$ & $24^{\circ} \mathrm{C}$ & 1049 & 0.949 & $18 \%$ \\
\hline $\begin{array}{c}\text { Tue } \\
26.06 \\
\end{array}$ & $13: 50$ & $37.1 \pm 18.9$ & $3.6 \mathrm{~m} / \mathrm{s}, 29^{\circ}(\mathrm{NNE})$ & $2.1 \mathrm{hrs}$ & $1.33,1.41$ & $24^{\circ} \mathrm{C}$ & 1049 & 0.990 & $8 \%$ \\
\hline $\begin{array}{c}\text { Wed } \\
27.06 \\
\end{array}$ & $13: 31$ & $49.5 \pm 23.2$ & $4.1 \mathrm{~m} / \mathrm{s}, 50^{\circ}(\mathrm{NE})$ & $1.1 \mathrm{hrs}$ & $1.25,1.29$ & $26^{\circ} \mathrm{C}$ & 1360 & 0.964 & $11 \%$ \\
\hline $\begin{array}{l}\text { Thu } \\
28.06\end{array}$ & $13: 12$ & $41.9 \pm 16.1$ & $6.0 \mathrm{~m} / \mathrm{s}, 54^{\circ}(\mathrm{NE})$ & $2.5 \mathrm{hrs}$ & $1.50,1.31$ & $26^{\circ} \mathrm{C}$ & 1967 & 0.962 & $10 \%$ \\
\hline
\end{tabular}




\subsubsection{Day-to-day and spatial variability in $\mathrm{NO}_{x}$ emissions}

We compare the TROPOMI $\mathrm{NO}_{x}$ emissions for Paris on clear-sky days to the TNOMACC-III (2011) adn EDGAR (2012) (Crippa et al., 2018) emisison inventories. The emissions are sampled for the same month, day of the week, and 12:00 hrs local time as the TROPOMI estimates. It is well known that Parisian $\mathrm{NO}_{x}$ emissions are dominated by traffic and heating (e.g. Shaiganfar et al. (2017), Kuenen et al. (2014)). As a result of tightening emission standards (Euro-IV, V, and VI norms) and the more modern vehicle fleet in 2018 compared to 2011 (Kuenen et al. (2014), EEA (2017)), we anticipate our TROPOMI estimates to be lower than the inventory estimates for 2011-2012. Figure 5.3 shows that the TROPOMI emissions for 2018 are (on average) 5-15\% lower than the inventory estimates for 2011-2012, but still a long way from the emissions projected for 2018 based on country-specific reductions for France (-26\% relative to 2011) (EEA, 2017).

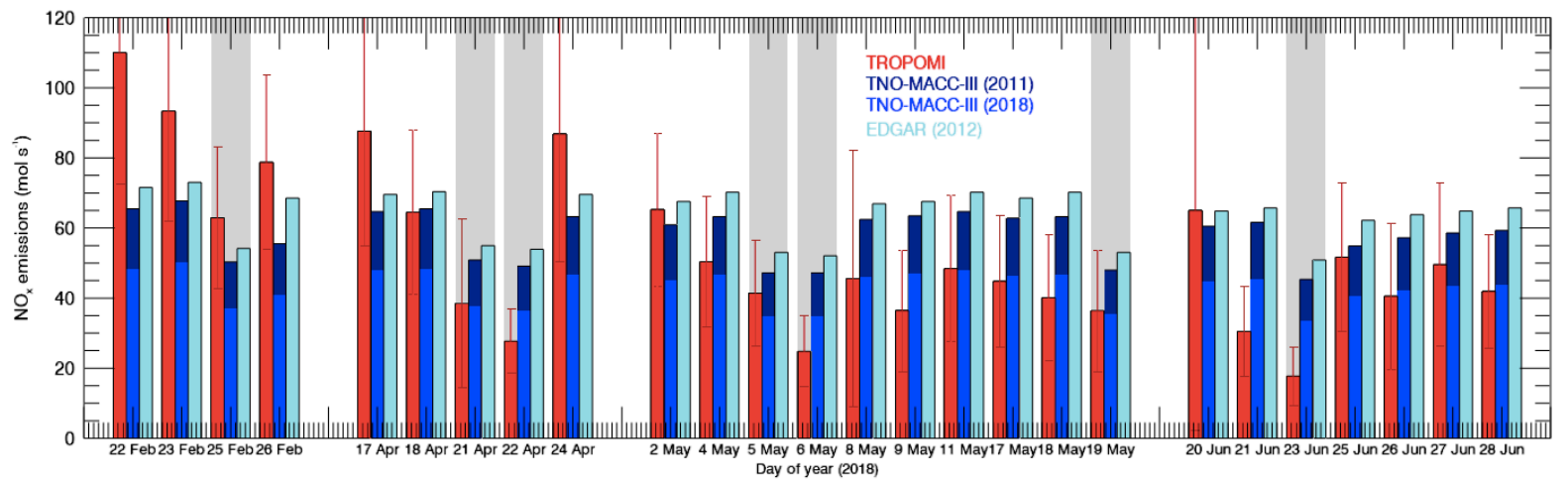

Figure 5.3: Time series of TROPOMI (red), TNO-MACC-III (2011: dark blue, 2018: blue), and EDGAR (2012) (light blue) $\mathrm{NO}_{x}$ emissions integrated over $60 \times 60 \mathrm{~km}^{2}$ around Paris. The medium blue bars represent projected TNO-MACC-III emissions for 2018 based on reductions of $26 \%$ for France between 2011 and 2018 predicted by the EEA. The grey shaded areas indicate weekend days. For days with two TROPOMI emission estimates, the mean is shown. Error bars correspond to 1-sigma uncertainties calculated via error propagation (Supplementary Material for Chapter 5).

In February 2018, a particularly cold month $\left(4^{\circ} \mathrm{C}\right.$ colder than normal), TROPOMI emissions are higher than those from the inventories. In April-June 2018, surface temperatures were above the $18^{\circ} \mathrm{C}$ threshold commonly assumed for turning on heating, and TROPOMI emissions are below those predicted by the inventories. This points to an underestimation of residential heating emissions in cold February, and an overestimate in the warm spring months, associated with the climatological rather than real-time activity factors for residential heating contributions assumed in the inventories. The TROPOMI emissions furthermore show a more pronounced weekend reduction $(35 \%)$ than the inventory $(21 \%)$. This is consistent with a smaller role for residential heating in April-June 
2018 than predicted by the inventory, and suggests that traffic emissions with pronounced weekend reductions dominate overall $\mathrm{NO}_{x}$ emissions in April-June. TROPOMI measurements are thus useful to not only evaluate absolute emissions, but also their temporal disaggregation into monthly, weekly, and diurnal estimates. These come with substantial uncertainties as they are based on behavioural patterns that are assumed to be the same across Europe. One example is that our emission estimates for Fridays are generally lower that those for other weekdays, in contrast to the predictions from the inventory, where Friday emissions are the highest of the week (Figure S5.4).

The noontime $\mathrm{NO}_{x}$ lifetimes from our method merely represent an improvement to prior, uncertain knowledge on $\mathrm{OH}$ concentrations from the CAMS model, constrained via the observed line densities. The lifetimes are about 11 hours in winter and 2-4 hours in spring (with typical uncertainties of $50 \%$ ), corresponding to mean $\mathrm{OH}$ concentrations between 1 and $12 \cdot 10^{6}$ molec. $\mathrm{cm}^{-3}$, consistent with other estimates (e.g. Shaiganfar et al. (2017), Beirle et al. (2011), Ren et al. (2003), Valin et al. (2013)). TROPOMI provides information on the sub-urban distribution of emissions in Paris. However, the spatial variability in our inferred $\mathrm{NO}_{x}$ emissions is similar to the a priori distribution from TNO-MACC-III (Figure S5.6).

\subsection{Discussion}

We show that the new TROPOMI $\mathrm{NO}_{2}$ measurements provide good quality information at a resolution unprecedented for satellite remote sensing observations. The new satellite measurements allow the determination of $\mathrm{NO}_{x}$ emissions from Paris on a day-to-day basis for cloudless spells. Our results indicate that $\mathrm{NO}_{x}$ emissions in 2018 are only $5-15 \%$ below inventory estimates for 2011-2012, falling short of anticipated reductions based on predicted improvements in technology and policies. This is most relevant on cold weekdays, when TROPOMI detects very high emissions compared to the inventories, pointing at strong contributions from the residential heating sector.

With the global coverage of TROPOMI measurements, emissions can be estimated for other major sources around the world in a consistent manner, as long as accurate knowledge of wind speed is available. The method is only weakly sensitive to assumptions on $\mathrm{NO}_{x}$ chemical lifetime for days with modest to strong wind speeds, when $\mathrm{NO}_{x}$ loss over the city is dominated by outflow. For days with stagnant air and large-scale subsidence the observed $\mathrm{NO}_{2}$ patterns provide a direct echo of the $\mathrm{NO}_{x}$ emission pattern over Paris. We conclude that the data and methodology presented here demonstrate the potential of TROPOMI and follow-up geostationary sensors to monitor emissions in ever greater spatial and temporal detail, not just for nitrogen oxides, but also for other air pollutants 
such as carbon monoxide, sulfur dioxide, and formaldehyde. TROPOMI data holds a strong promise for the detection of emissions also from smaller cities and point sources, and is particularly interesting for still uncharted sources.

\subsection{Methods}

\subsubsection{TROPOMI sensor}

$\mathrm{NO}_{2}$ tropospheric columns are provided by the TROPOspheric Monitoring Instrument (TROPOMI) on board of the Copernicus Sentinel 5 Precursor (S5P) satellite (Veefkind et al., 2012). We use level-2 data (version 1.0.2) processed by KNMI and DLR for February-June 2018 that are available to validation teams at https://scihub.copernicus.eu. The first 6 months of the mission were used for special observations to commission the satellite and the ground processing systems; the operational phase started in April 2018. The instrument measures the top of the atmosphere solar radiation reflected by and radiated from the Earth between 270-500 nm and 675-775 nm, and in the short-wave infrared. The instrument images a strip of the Earth on a 2-D detector for a period of 1 second during which the satellite moves by about $7 \mathrm{~km}$. This strip has dimensions of approximately $2600 \mathrm{~km}$ across the track direction of the satellite and $7 \mathrm{~km}$ in the along-track direction. The two dimensions of the detector allow to simultaneously measure 450 spectra over the entire $2600 \mathrm{~km}$ strip, corresponding to a spatial resolution of the $7 \mathrm{~km}$ (along) $\times 3.5 \mathrm{~km}$ (across) at nadir. The equator crossing time is near 13:40 local solar time, which results occasionally in two overpasses over Paris on the same day, with some 100 minutes in between measurements.

\subsection{2 $\quad \mathrm{NO}_{2}$ retrieval algorithm}

The $\mathrm{NO}_{2}$ columns are retrieved with a 3-step procedure performed for each measured level1b spectrum as described in the Algorithm Theoretical Baseline Document (van Geffen et al., 2018). In the first step, $\mathrm{NO}_{2}$ slant column densities are obtained from the radiance and irradiance spectra using the Differential Optical Absorption Spectroscopy technique in the 405-465 $\mathrm{nm}$ window where $\mathrm{NO}_{2}$ has prominent spectral features. Then, the slant column is separated into a stratospheric and tropospheric part based on information from a data assimilation system. Finally, the tropospheric slant columns are converted into tropospheric vertical column densities by application of a tropospheric air mass factor (AMF) based on a look-up table of altitude-dependent AMFs and actual information on surface and cloud characteristics and on the vertical distribution of $\mathrm{NO}_{2}$ predicted by the TM5-MP model on a $1^{\circ} \times 1^{\circ}$ grid (Williams et al., 2017). 
The TROPOMI retrieval builds on principles used in the DOMINO v2 approach (Boersma et al., 2011), but includes many retrieval improvements proposed within the European Union Quality Assurance for Essential Climate Variables (QA4ECV) project (Boersma et al. (2018), Lorente et al. (2017)). These include an improved wavelength calibration and the inclusion of $\mathrm{O}_{2}-\mathrm{O}_{2}$ and liquid water in the DOAS fitting model (van Geffen et al. (2015), Zara et al. (2018)). In combination with the high signal-to-noise ratio of TROPOMI, this leads to lower uncertainties in the slant columns $\left(0.5-0.6 \cdot 10^{15}\right.$ molec $\left.\cdot \mathrm{cm}^{-2}\right)$ compared to OMI. The data assimilation approach to estimate the stratospheric $\mathrm{NO}_{2}$ columns is based on the TM5-MP model and operates on a $1^{\circ} \times 1^{\circ}$ grid (Williams et al., 2017). Stratospheric $\mathrm{NO}_{2}$ in TM5-MP (free running mode) is driven by nudging to satellite-observed climatological $\mathrm{HNO}_{3}: \mathrm{O}_{3}$ ratios (from ODIN and HALOE) and 3-hourly ECMWF analysed and forecast meteorological fields. The TM5-MP simulations are updated every 30 minutes based on TROPOMI slant columns available in that time-step, and the forecast $\mathrm{NO}_{2}$ field is then used to estimate the stratospheric $\mathrm{NO}_{2}$ column. A preliminary comparison with ground-based NDACC SAOZ measurements suggests agreement to within $10 \%$ (or $0.3 \cdot 10^{15} \mathrm{molec} \cdot \mathrm{cm}^{-2}$ ) between the TROPOMI and SAOZ stratospheric $\mathrm{NO}_{2}$ columns (Compernolle et al., 2018). The air mass factor that converts the slant column density to vertical column density is calculated using the radiative transfer model Doubling-Adding code KNMI. The AMF includes a correction factor to account for atmosphere's sphericity calculated with the 3D model McArtim (Lorente et al., 2017). This correction affects mainly the stratospheric AMFs that are highly relevant in the stratospheric $\mathrm{NO}_{2}$ estimation, and results in lower stratospheric $\mathrm{NO}_{2}$ columns especially for extreme solar zenith angles in the winter hemisphere (Boersma et al., 2018). The a priori $\mathrm{NO}_{2}$ vertical profiles from TM5-MP have an improved spatial resolution with respect to DOMINO.

\subsubsection{TROPOMI $\mathrm{NO}_{2}$ data filtering}

We use tropospheric $\mathrm{NO}_{2}$ columns measured from orbits with cloud radiance fractions less than 0.5, corresponding to geometric cloud fractions of up to 0.2 , over Paris, as recommended in the TROPOMI ATBD (van Geffen et al., 2018). Experience with previous satellite $\mathrm{NO}_{2}$ observations showed that measurements with the lowest effective cloud fractions compare most favourably with independent measurements (Schaub et al., 2006), and are of the highest quality (Lorente et al., 2017). Following this criterion rendered a total of 36 orbits (obtained on 29 different days) with a mostly unobstructed view on Paris, corresponding to a retention rate of approximately $25 \%$ in the February-June 2018 period.

Arguably the largest source of uncertainty in the satellite retrievals is the computation of the tropospheric AMF (Boersma et al. (2018), Lorente et al. (2017)). We evaluated the 
influence of cloud parameters (effective cloud fraction and cloud pressure) on the AMF patterns, and found that in some situations the rather course-gridded surface albedo climatology used in the FRESCO+ cloud pressure retrieval, led to spurious jumps in the tropospheric air mass factors for some days, which were absent when evaluating the tropospheric slant column divided by the geometrical air mass factor. Based on these tests, we rejected a number of orbits from further analysis: 24 February (orbits 1902 and 1903). This was also a day with relatively high cloud radiance fractions over Paris. We also rejected data from 20 April 2018, as on this day various pixels right in the middle of the pollution build-up (13 out of 126) were classified as non-valid over Paris. A day with rapidly changing wind direction (19 April) was not considered either in our analysis. There were strong indications for winds shifting direction and speed in the hours just before the TROPOMI overpass time (Venstusky, 2018) of 13:22 UTC.

Our validation exercise over Paris suggests that TROPOMI $\mathrm{NO}_{2}$ columns have a low bias with a multiplicative component of $25 \%$ (Figure S5.1). This is in line with other validation activities that suggest that TROPOMI $\mathrm{NO}_{2}$ columns are on average some 20-40\% lower than co-located $\mathrm{NO}_{2}$ columns measured with independent ground-based measurements 34. This could be indicative of a low bias in the satellite retrievals or a high bias in the ground-based measurements, but could also indicate differences in spatial representativeness between the ground-based and satellite measurements. An experiment in which we recalculated TROPOMI air mass factors by replacing a priori assumptions on the $\mathrm{NO}_{2}$ vertical distribution (from TM5-MP) by profile shapes simulated with a highresolution model, shows higher $\mathrm{NO}_{2}$ columns, and suggests stronger increases of $\mathrm{NO}_{2}$ along with the wind from TROPOMI.

\subsubsection{Line densities}

For each day, the tropospheric $\mathrm{NO}_{2}$ columns are converted into one-dimensional line densities along the wind direction over Paris. We do so by gridding the original TROPOMI field to a $0.05^{\circ} \times 0.05^{\circ}$ grid rotated towards the mean wind direction in the boundary layer (Figure S5.3). We then integrate this rotated field over a $60 \mathrm{~km}$ interval perpendicular to the wind direction, in units of molecules $\cdot \mathrm{cm}^{-1}$. The $60 \mathrm{~km}$ interval is motivated by the spatial contours of Paris and the horizontal distribution of bottom-up emissions that suggest that the entire Paris metropolitan area is enveloped in all directions within a radius of $30 \mathrm{~km}$ (Fig. S5.2), and so avoids interference from other sources. We focus on the pollution build-up over the city along with the wind. Our line densities thus start 30 $\mathrm{km}$ upwind of the Paris city centre $(\mathrm{x}=0 \mathrm{~km})$, and end $30 \mathrm{~km}$ downwind $(\mathrm{x}=60 \mathrm{~km})$. This ensures that they capture the full extent of the accumulation of $\mathrm{NO}_{2}$ over the emitting metropolitan area. 


\subsubsection{Boundary layer information on wind and chemistry}

We calculate mean boundary layer wind speed at 12 UTC (13:00 hrs local time in Paris) from ECMWF data. The wind data is taken from the 6-hourly data ECMWF ERAInterim reanalysis (e.g. Dee et al. (2011), Berrisford et al. (2011)). The original horizontal resolution of this dataset is about $80 \mathrm{~km}$, we use a re-gridded version at $0.125^{\circ} \times 0.125^{\circ}$. At the TROPOMI overpass time, the boundary layer is usually well mixed, so the boundary layer average wind speed and wind direction is a good representation of the transport of pollutants within the Paris dome. We ensured that the $\mathrm{NO}_{2}$-weighted mean boundary layer wind speed did not differ significantly from the mean wind speed between the surface and the boundary layer depth.

The a priori $\mathrm{NO}_{x}$ lifetime is calculated as:

$$
\tau_{N O_{x}}=\frac{\left[N O_{x}\right] /\left[N O_{2}\right]}{k[O H][M]}
$$

with $\left[\mathrm{NO}_{x}\right],\left[\mathrm{NO}_{2}\right]$, and $[\mathrm{OH}]$ the boundary layer mean concentrations simulated by CAMS at 12 UTC over Paris, and k[M] the high-pressure, high-temperature reaction rate constant for oxidation of $\mathrm{NO}_{2}\left(2.6 \cdot 10^{-11} \mathrm{~cm}^{3}\right.$ molec $\left.^{-1} \mathrm{~s}^{-1}\right)$. The CAMS global near-real time atmospheric composition reanalysis provides 3-hourly information of atmospheric composition, with a horizontal resolution of $40 \mathrm{~km}$. The CAMS system uses satellite observations of atmospheric composition in its 4-D variational data assimilation system (Inness et al., 2015), together with the Integrated Forecast System for atmospheric composition (C-IFS) from ECMWF 37 (Flemming et al., 2015).

\subsubsection{Simulating line densities with a superposition model}

We constructed a simple model that simulates the $\mathrm{NO}_{2}$ line density as a function of the along-wind distance $\mathrm{x}$ over Paris. This model simulates the build-up of $\mathrm{NO}_{2}$ in the air column as a superposition of line densities caused by the emissions in cell $\mathrm{i}$. The prior emission in these cells is from the TNO-MACC-III $\mathrm{NO}_{x}$ emissions, integrated cross-track over the full $60 \mathrm{~km}$ width perpendicular to the wind over Paris (unit molecules $\mathrm{NO}_{x}$ $\left.\mathrm{cm}^{-1} \mathrm{~s}^{-1}\right)$ ). For each $5 \mathrm{~km}$ long cell i between $0 \mathrm{~km}<\mathrm{x}<60 \mathrm{~km}$ the contribution to the line density downwind of the cell is calculated using a simple column model (Jacob, 1999):

$$
N_{i}(x)= \begin{cases}\frac{E\left(x_{i}\right)}{k}\left(1-\exp \left[-k\left(x-x_{i}\right) / u\right]\right) \frac{\left[N O_{2}\right]}{\left[N O_{x}\right]} & x \geq x_{i} \\ 0 & x<x_{i}\end{cases}
$$


where $\mathrm{N}_{i}(\mathrm{x})$ represents the contribution from $\mathrm{E}\left(\mathrm{x}_{i}\right)$, the $\mathrm{NO}_{x}$ emissions from cell $\mathrm{x}_{i}$ alone, to the overall line density, $\mathrm{k}$ is the loss rate constant of $\mathrm{NO}_{x}$ for daytime conversion to nitric acid and $u$ is the vertically averaged boundary layer wind speed. In Eq. (1), scaling with the vertically averaged $\left[\mathrm{NO}_{2}\right]:\left[\mathrm{NO}_{x}\right]$ mixing ratio is required to express the line densities in terms of $\mathrm{NO}_{2}$. The superposition accounts for the spatially varying emission rates $(\mathrm{E}(\mathrm{x}))$ in the urban area and reads:

$$
N(x)=\sum_{i} N_{i}(x)
$$

with $\mathrm{N}(\mathrm{x})$ describing the build-up of $\mathrm{NO}_{2}$ in molecules $\mathrm{cm}^{-1}$ along with the wind exclusively over the Paris Metropolitan Area. The build-up reflects the underlying emission strength and pattern $\mathrm{E}(\mathrm{x})$ and is influenced by the first order chemical loss and wind speed over the city. The background value at the upwind end of the city $(\mathrm{x}=0 \mathrm{~km})$ is assumed to be representative for the entire city, so that the increase between 0 and $60 \mathrm{~km}$ is attributed exclusively to emissions from the city.

\subsubsection{Daily $\mathrm{NO}_{x}$ emissions over Paris from the TNO-MACC-III inven- tory}

To obtain the emission for a particular day at 12:00 hrs (just before the TROPOMI overpass time), the 24-hour mean emissions for Paris were first integrated over a $60 \times 60$ $\mathrm{km}^{2}$ area around the city centre, and then scaled by specific monthly, weekly, and diurnal factors from the TNO-inventory. Figure S5.4 illustrates the temporal scaling factors from the TNO MACC-III inventory, with the highest emissions in February and March, and lowest in June. The weekly cycle in emissions peaks on Friday, and has some $21 \%$ lower emissions on weekend days. The diurnal cycle indicates that emissions at 12:00 hrs are 20-30\% higher than the 24-hour mean. These factors from a TNO report (Denier van der Gon et al., 2010) have a high uncertainty embedded. For instance, the diurnal cycle in emissions from road transport is based on traffic intensity time series from 1985 to 1998 in the Netherlands. It seems plausible that not only traffic intensity and emission abatement in cars decreased in the past decades, but also that the temporal variation in Paris is different than in the Netherlands. 



\section{General discussion and outlook}

In this chapter we place the results and conclusions of the main chapters within the perspective of future research.

\subsection{Radiative transfer and air mass factor calcula- tion}

The calculation of the air mass factor is the most uncertain step of the $\mathrm{NO}_{2}$ retrieval algorithm. We have seen in Chapter 2 and 3 that the structural uncertainty can be estimated by applying different methodologies to the same data and analysing the spread in the results. In this thesis we addressed the air mass factor (AMF) structural uncertainty by comparing different AMF calculation methods by different retrieval groups. We analysed in detail all the sub-processes in the AMF calculation, which allowed us to simultaneously address the following research questions:

- How can we improve the air mass factor calculation process?

- What are the main sources of the AMF structural uncertainty, and can we quantify these uncertainties for every step of the calculation?

The detailed comparison between different approaches pointed to possible improvements in the AMF calculation process. The box-AMF comparison showed that McArtim model simulates systematically lower box-AMFs in the stratosphere as compared to DAK and the other radiative transfer models. We attributed these differences to the model's geometrically more realistic description of photon scattering in a spherical atmosphere. Based on this result, we included a correction for sphericity in the altitude dependent air mass factor look-up table and in the data assimilation for the stratosphere-troposphere separation. This correction, together with the use of a higher resolution a priori profile, resulted in (compared to DOMINO v2) lower (5-20\%) tropospheric $\mathrm{NO}_{2}$ columns over polluted regions that show a better agreement with independent ground-based measurements. The comparison against DOMINO and the different validation exercises (e.g. over 
Tai'an and De Bilt in Chapter 2) show that the AMF calculated as a result of the research in Chapter 3 and the QA4ECV project represent an improvement w.r.t. previous AMFs.

In the tropospheric AMF comparison between groups, we identified the main sources of structural uncertainty in every step of the AMF calculation and we traced back these uncertainties to their underlying causes. When retrieval groups used their preference for ancillary data along with their preferred cloud and aerosol correction, we estimated the overall structural uncertainty of the AMF calculation to be up to $42 \%$ over polluted regions. The steep increase from the structural uncertainty in top-of-atmosphere (TOA) reflectance simulations of $6 \%$ to $42 \%$ strongly suggests that it is not the models or the calculation method but the assumptions and choices made to represent the state of the atmosphere that introduce most structural uncertainty in the AMF calculation.

The most critical assumptions (i.e. strongest source of structural uncertainty) for the AMF calculation are the choice for a specific cloud correction and aerosol correction, followed by the choice of a priori profiles and surface albedo. Therefore future research should focus on improving cloud and aerosol characterization for trace gas retrievals. For clouds, their structure and radiative properties can be more realistically simulated using 3 -D radiative transfer models, and incorporate these to the cloud model used in the air mass factor calculation. The implicit aerosol correction should be further developed by including a (better) physical aerosol characterization in the radiative transfer. Recent studies by Liu et al. (2019) and Chimot et al. (2018) attempted to improve the aerosol correction by using aerosol properties retrieved from Moderate Resolution Imaging Spectroradiometer (MODIS), Cloud-Aerosol Lidar with Orthogonal Polarization (CALIOP) and Ozone Monitoting Instrument (OMI) satellites. Liu et al. (2019) showed that the new correction, which constraints the GEOS-Chem modelled aerosol parameters using aerosol extinction profiles from CALIOP, improved the Peking University OMI NO 2 (POMINO) retrieval over China in hazy conditions. Chimot et al. (2018) applied the aerosol correction using aerosol parameters (aerosol layer height and aerosol optical thickness) retrieved from OMI. They showed the feasibility of their methods for cloud-free scenes with high aerosol load and in the presence of strongly absorbing aerosols, and recommended to use the explicit aerosol correction when a clear distinction between clouds and aerosols cannot be made. They highlighted that difficulties still exist in the application of the implicit correction due to the interplay on the radiative transfer of the $\mathrm{NO}_{2}$ profile shape, aerosol properties and surface reflectance.

The use of high resolution (e.g. about $15 \times 15 \mathrm{~km}^{2}$ ) a priori profiles improves the AMFs and hence $\mathrm{NO}_{2}$ satellite retrievals, as urban and background areas are better resolved (e.g. McLinden et al. (2014), Heckel et al. (2011)). Although it can be computationally expensive to apply these profiles in a global retrieval, with the new and future high resolution 
instruments to use high resolution prior information will be necessary . There are stateof-the-art chemistry transport models than can model global $\mathrm{NO}_{2}$ fields at high spatial resolution (e.g. WRF-Chem, GEOS-Chem). Regionally for Europe, the Copernicus Atmosphere Monitoring System CAMS provides $\mathrm{NO}_{2}$ fields from an ensemble of 7 chemistry transport models at a resolution of $40 \times 40 \mathrm{~km}^{2}$ that can be used to re-calculate the AMFs. The need of using high resolution prior information also applies to the surface albedo: climatologies at coarse resolution currently used in the $\mathrm{NO}_{2}$ retrievals degrade the intrinsic spatial resolution of the scenes measured at high resolution, like with TROPOMI. Although TROPOMI itself will soon provide a surface reflectance "climatology" as its predecessors GOME-2 and OMI, other alternatives should be investigated. The ADAM (A surface reflectance DAtabase for ESA's earth observation Missions) database provides monthly climatologies of surface reflectance at $0.1 \times 0.1^{\circ}$ resolution derived from MODIS, which might be an alternative to current coarse resolution climatologies. The QA4ECV project also derived a land surface albedo climatology (1982-2016) based on several satellite instrument that, combined with another source of information for ocean reflectance, could be used in current $\mathrm{NO}_{2}$ retrievals. Until now these options have been discarded because of the fact that different satellite would then provide the ancillary information, which leads to a less consistent cloud and $\mathrm{NO}_{2}$ retrieval framework (e.g. different instrumental features, different spectral range and radiative transfer characterization and atmospheric correction).

Structural uncertainty is intrinsic to the retrieval algorithm formulation and it is considered to be a source of systematic uncertainty. Currently, most satellite retrievals only consider the theoretical uncertainty. However, uncertainty from methodological differences should also be considered when estimating the overall uncertainty. In case of the $\mathrm{NO}_{2}$ and formaldehyde (HCHO) retrievals, the magnitude of the structural uncertainty is significant and comparable to the theoretical uncertainty. In the QA4ECV and TROPOMI HCHO retrieval algorithms, structural uncertainty has been accounted for as an additional contribution in the total error calculation of the AMF (De Smedt et al., 2018). Even though this results in a more exhaustive uncertainty budget estimation, the fact that the calculation of the theoretical and structural uncertainty share several components can influence the overall uncertainty estimation and needs to be further investigated.

Chapter 3 provides evidence of the need for improvement of the different ancillary data sets and their uncertainty estimates. Because there is not a "true" air mass factor value to use as a reference, direct validation of the AMF using independent measurements is not possible, only via validation of the vertical columns. Therefore there is a strong need of having dedicated validation exercises against independent data focusing on situations where AMF structural uncertainty has the highest impact: strong pollution events and 
scenes with residual clouds. The ancillary data itself $\left(\mathrm{NO}_{2}\right.$ profile, cloud and aerosols distributions and surface albedo) are in deep need of validation and should also be considered when designing future validation campaigns.

\subsection{Surface reflectance anisotropy}

Surface reflectance anisotropy (i.e. the angular distribution of the light reflected by a surface) has implications for satellite retrievals of albedo, clouds and trace gases such as $\mathrm{NO}_{2}$. Because most of the operational retrievals assume that the surface reflects light isotropically, in Chapter 4 we addressed the following research questions:

- How accurate is the use of Lambertian surface albedo for backward and forward scatter viewing geometries from different satellite instruments?

- How can we account for surface reflectance anisotropy in the radiative transfer model DAK?

- How does surface reflectance anisotropy affect cloud retrievals and $\mathrm{NO}_{2}$ retrievals via clear sky and cloudy sky contributions to the tropospheric AMF?

In Chapter 4 we have shown that surface reflectance anisotropy needs to be taken into account in a coherent manner for more realistic and accurate satellite retrievals of clouds and $\mathrm{NO}_{2}$. We showed that retrievals suffer from systematic errors when surface reflectance anisotropy effects are not taken into account. We found biases in cloud fractions across the orbit of up to $50 \%$ in the GOME-2 FRESCO cloud algorithm and up to $26 \%$ in the OMI OMCLDO2 cloud algorithm. The biases are larger over vegetation and forested areas in the near-infrared, and weaker but still relevant in the visible, where surfaces are darker and Rayleigh scattering effects are stronger. These biases are caused by systematic under and over estimation of the surface reflectance by the surface LER (Lambertian Equivalent Reflector) climatologies that propagates into the cloud retrieval. The effects on clouds are highly relevant for scenes with low cloud fractions, where trace gas retrievals are still sensitive to pollution close to the ground. Surface reflectance anisotropy affects both clear-sky AMF and cloud fractions, which in combination significantly affect $\mathrm{NO}_{2} \mathrm{AMFs}$. The combined effect, stronger than the effect of either contribution alone, is that $\mathrm{NO}_{2}$ AMFs in polluted situations vary up to $\pm 25 \%-35 \%$. The sign and magnitude of surface BRDF effects show strong spatial variations and depend on cloud fraction and cloud pressure.

A natural progression of this work would be to substitute the current surface LER climatologies by a better surface reflectance characterization which is particularly urgent for new and future satellite instruments measuring at high resolution with a wider swath. 
An alternative to the traditional surface LER climatology is the 'directional surface LER climatology'. This climatology is constructed in the same way as traditional climatologies (i.e. the surface reflectance is still considered isotropic) but discretizes the orbit swath according to the different relative viewing geometries, which results in a viewing geometry dependent surface LER climatology. Another alternative is the so-called geometry-dependent LER (GLER)(Vassilkov et al. (2017)). In this approach spatially averaged MODIS BRDF information over each OMI pixel is used to construct a LER database that is viewing geometry dependent. The advantage of using the directional LER and GLER is that current algorithms only need minor adjustments to adapt to the new datasets, mainly because these are derived for the same pixel sizes, geometries and wavelengths and using the same retrieval framework as the one used in cloud and $\mathrm{NO}_{2}$ retrievals. The alternative, the use of a full surface BRDF implementation, is preferred but needs major changes to the algorithm framework. For instance, it would be necessary to include two more dimensions in the LUT to account for the different scattering types. A full BRDF implementation also implies the use of external BRDF parameters from different satellite instruments (e.g. MODIS), which gives less consistency to the retrievals as the spectral ranges, radiative transfer and atmospheric corrections differ for the retrievals of the different satellite products. The cloud fraction dependency with the viewing geometry for cloudy scenes also suggests that the use of a more realistic cloud model should be explored in further research for the improvement of cloud retrievals. A first step forward from the currently used Lambertian model would be to consider in the radiative transfer a vertical structure of different scatterers within the vertical extension of the clouds and to account for directionality in the scattering of light by clouds.

Chapter 4 raised the need of a thorough analysis of how cloud parameters retrieved with current cloud retrievals depend on geometry to estimate the effects at larger time and spatial scales. Retrieved cloud fractions and cloud pressures suffer from a geometry dependent bias that might not be visible on a day to day basis, but does appear when analysing the cloud products at longer periods over specific locations as we do in Chapter 4. A preliminary analysis with 5 days of global GOME-2B data over land showed that using the directional LER in the FRESCO retrieval reduces the cloud pressure by $40 \mathrm{hPa}$ on average. This analysis also showed a decrease in cloud fractions by up to 0.1 on the part of the orbit that measures under backward-scatter geometries, where currently FRESCO retrieves hardly any cloud fractions below 0.2 . This threshold of 0.2 is highly relevant for $\mathrm{NO}_{2}$ and other trace gas retrievals (i.e. $\mathrm{HCHO}, \mathrm{SO}_{2}$, fluorescence) as it is used to decide on whether a pixel is considered cloud-free or not. This implies that there might be $\mathrm{NO}_{2}$ retrievals considered cloud contaminated when in reality it could be a clear-sky scene, which brings up the need to also investigate the number of pixels that might be (and might have been) compromised by this. 
Although in Chapter 4 we have shown that it is necessary to coherently account for surface reflectance anisotropy effects, the surface BRDF effects have not yet been applied to a complete global cloud and $\mathrm{NO}_{2}$ retrieval. This should be a priority for current retrievals that still consider the surface as a Lambertian reflector. This would result in improved cloud and trace gas retrievals, especially for highly polluted scenes with residual clouds, for measurements under geometries more affected by surface anisotropy (i.e. backward scattering) and over areas covered by vegetation. Besides the surface reflectance characterization, ocean reflectance anisotropy also has to be taken into account, as cloud and trace gases are also retrieved over ocean scenes. The ocean reflectance can be parametrized, similar to surface BRDF, using the Cox-Munk model that simulates the reflection as a function of geometry and wind speed (Cox and Munk, 1954). Additionally, two terms to account for foam reflectance (Koepke, 1984) (as a function of wind speed) and ocean column reflectance (Morel, 1988) (as a function of chlorophyll concentrations) can also be added.

\subsection{TROPOMI $\mathrm{NO}_{2}$ measurements for daily $\mathrm{NO}_{x}$ emission estimates}

The research described in Chapter 5 started just a month after TROPOMI was launched in October 2017. Data from the first month of TROPOMI measurements showed pollution from sources with unprecedented spatial resolution. Its predecessors (e.g. OMI) could only come close by temporally averaging, and not exclusively with only one satellite overpass. The $22^{\text {nd }}$ of November 2017, a day with mostly clear skies over Europe, TROPOMI $\mathrm{NO}_{2}$ measurements showed a plume from Paris that was transported towards the north-east more than $100 \mathrm{~km}$ away. With this picture as a motivation for our study, and with a constant flow of new TROPOMI data coming in, we focused on answering the following questions:

- How is the quality of the TROPOMI $\mathrm{NO}_{2}$ retrieval algorithm and of the high resolution $\mathrm{NO}_{2}$ measurements?

- Can we estimate daily $\mathrm{NO}_{x}$ emissions from TROPOMI $\mathrm{NO}_{2}$ measurements focusing on the build up of pollution within the city?

- How robust is the method applied to a megacity like Paris?

In Chapter 5 we show that is it possible to estimate daily $\mathrm{NO}_{x}$ emissions of the Paris metropolitan area from the $\mathrm{NO}_{2}$ measurements of TROPOMI. The high resolution and improved signal-to-noise ratio of TROPOMI allows to apply a simple column model focusing on the build up of pollution within the city. Our methodology is inspired by the 
one in Beirle et al. (2011) but it differs from it as it overcomes some limitations from the assumptions in the downwind decay of the plume and the use of coarser resolution $\mathrm{NO}_{2}$ measurements from OMI. TROPOMI allows the use of daily measurements regardless of the wind direction and without the need to average $\mathrm{NO}_{2}$ tropospheric columns. Averaging introduces errors as it implies the interpretation of different plumes transported in different days with changing atmospheric conditions (e.g. wind speed and direction). On the other hand, we focus on the build up of $\mathrm{NO}_{2}$ over the source (i.e. the city) without analysing the downwind decay to estimate the lifetime of $\mathrm{NO}_{2}$. In the high resolution daily measurements of TROPOMI, the determination of the lifetime from the downwind decay is influenced by the diurnal cycle of emissions, the downwind emission sources and the variability of the lifetime within the plume (de Zeeuw, 2018). When these effects are neglected in the column model, the lifetime inferred from the downwind decay is underestimated, thus the downwind decay of the plume is not a good constraint on the $\mathrm{NO}_{x}$ lifetime as in (Beirle et al., 2011). Because an accurate determination of the lifetime is not possible without accounting for these effects, we focus on the accumulation of $\mathrm{NO}_{2}$ within the metropolitan area of Paris where most of the $\mathrm{NO}_{x}$ emissions take place. The accumulation of $\mathrm{NO}_{2}$ first upwind and then within the city reflects the spatial distribution of emissions within the $60 \times 60 \mathrm{~km}^{2}$ metropolitan area of Paris. With an overpass time of 13:30 LT, the TROPOMI emission estimates are representative for the noon-time emissions for the entire metropolitan area. The method relies on the knowledge of wind speed and direction to good accuracy, and it is only weakly sensitive to assumptions on $\mathrm{NO}_{x}$ chemical lifetime for days with modest to strong wind speeds, when $\mathrm{NO}_{x}$ loss over the city is dominated by outflow.

The TROPOMI $\mathrm{NO}_{2}$ retrieval is based on the principles used in the DOMINO v2 retrieval for OMI satellite and includes improvements developed for the QA4ECV retrieval algorithm (discussed in Chapter 2). However, the unprecedented high resolution of TROPOMI measurements brings new challenges for the retrieval community. Apart from analysing the tropospheric $\mathrm{NO}_{2}$ columns from TROPOMI, we also analysed the slant column densities divided by the geometrical AMF to identify artificial patterns in the AMF (caused by the ancillary datasets) that could be affecting the retrieved $\mathrm{NO}_{2}$ vertical columns. During some of the days the cloud parameters were causing spurious jumps in the AMF (visible in the $\mathrm{NO}_{2}$ fields), and we traced these jumps back to the interaction of the cloud retrieval with the surface albedo dataset, which was not properly being interpolated in space ${ }^{1}$. The surface albedo climatologies used in the cloud and $\mathrm{NO}_{2}$ retrieval are based on measurements from GOME-2 and OMI respectively, and they are given in a rather coarse $\left(1^{\circ} \times 1^{\circ}, 0.5^{\circ} \times 0.5^{\circ}\right)$ spatial resolution (Kleipool et al. $(2008)$, Tilstra et al. (2017)). Retrieval groups need to investigate the representation of the sur-

\footnotetext{
${ }^{1}$ This issue has been solved by the retrieval team and corrected in the updated version of the cloud algorithm.
} 
face reflectance in order to perform accurate retrievals of clouds and $\mathrm{NO}_{2}$ from TROPOMI and future high resolution sensors (as discussed in previous section).

The a priori profiles used in the retrievals are from the TM5-MP model at $1^{\circ} \times 1^{\circ}$ resolution, an improvement compared to the $3^{\circ} \times 2^{\circ}$ resolution used in DOMINO. However, this resolution is still too coarse for the high resolution of TROPOMI $\mathrm{NO}_{2}$ measurements at $3.5 \times 7 \mathrm{~km}^{2}$, and the contrast between polluted and background areas is insufficiently represented in the AMF patterns. When using a coarse resolution model for the a priori profiles, background $\mathrm{NO}_{2}$ values are typically overestimated and in high pollution spots underestimated. We performed an experiment and re-calculated the AMFs using higher resolution a priori profiles from CAMS for 4 days (Henk Eskes and John Ntouros, KNMI, 2018). The results showed an underestimation of the $\mathrm{NO}_{2}$ columns within the plumes from Paris of around $10 \%-15 \%$, and an similar overestimation in the background $\mathrm{NO}_{2}$ columns outside the plumes. TROPOMI $\mathrm{NO}_{2}$ being biased low within the polluted Paris is in line with the conclusions from the first validation results. This could be attributed not only to the resolution of the a priori profile, but also to the different emissions or vertical mixing in the models. This directly points to the need of thorough validation campaigns with independent measurements (e.g. MAX-DOAS, surface and aircraft measurements) to validate the different sources of a priori profiles for the retrievals. In Chapter 5 we have scaled the emissions based on the validation of TROPOMI $\mathrm{NO}_{2}$ with ground based measurements from the Airparif network, but the use of high-resolution profile shapes should be considered in future applications of TROPOMI $\mathrm{NO}_{2}$ measurements.

Chapter 5 shows that it is necessary to monitor emissions in real time and presents TROPOMI $\mathrm{NO}_{2}$ measurements as an additional tool to bottom-up emission inventories, as they provide $\mathrm{NO}_{x}$ emission estimates at sub-urban scales and on a day-to-day basis. It is at these temporal and spatial scales where bottom-up emission inventories are less accurate as they are based in rather general assumptions. The method presented in Chapter 5 can be easily applied to other megacities: within the Paris area TROPOMI makes enough measurements to calculate the line density with 12-15 points along the city every day, so we think that the application of the method to smaller sources is also feasible. The challenge now is to automatize the process so we can derive emissions as soon as TROPOMI $\mathrm{NO}_{2}$ measurements and accurate wind speed and wind direction information are available. 


\section{Summary}

Nitrogen oxides $\left(\mathrm{NO}_{x}=\mathrm{NO}+\mathrm{NO}_{2}\right)$ play an important role in atmospheric chemistry, therefore affecting air quality and Earth's radiative forcing, which impact public health, ecosystems and climate. Remote sensing from satellites in the ultraviolet and visible (UV-Vis) spectral range results in measurements of tropospheric $\mathrm{NO}_{2}$ column densities with high spatial and temporal resolution that allow, among many applications, to monitor $\mathrm{NO}_{2}$ concentrations and to estimate $\mathrm{NO}_{x}$ emissions. $\mathrm{NO}_{2}$ satellite retrievals have improved extensively in the last decade, together with the increased need of having traceable characterization of the uncertainties associated with the $\mathrm{NO}_{2}$ satellite measurements. The spatial resolution of the satellite instruments is improving such that the observed $\mathrm{NO}_{2}$ pollution can now be traced back to emissions from individual cities, power plants, and transportation sectors. However, the uncertainty of satellite $\mathrm{NO}_{2}$ retrievals is still considerable and mainly related to the adequacy of the assumptions made on the state of the atmosphere. In this thesis we have improved the critical assumptions and our understanding in the radiative transfer modelling for $\mathrm{NO}_{2}$ satellite measurements, and we use the new TROPOMI $\mathrm{NO}_{2}$ measurements to quantify daily $\mathrm{NO}_{x}$ emissions from a single urban hot spot.

In the Quality Assurance for Essential Variables (QA4ECV) project, we developed a community $\mathrm{NO}_{2}$ and formaldehyde (HCHO) retrieval algorithm based on best practices of different state-of-the-art retrieval algorithms (Chapter 2). To develop the QA4ECV algorithm, a systematic analysis of the three retrieval steps was performed, comparing different approaches by leading retrieval groups to create an improved retrieval algorithm. By applying it to four different sensors we have retrieved more than 20 years of $\mathrm{NO}_{2}$ and $\mathrm{HCHO}$ measurements in a consistent way, both at algorithm level using the same methodology and assumptions and across timescales.

The QA4ECV $\mathrm{NO}_{2}$ retrieval incorporates relevant improvements and it also provides a better quantification and understanding of the uncertainties in all the retrieval steps. The spectral fitting method now accounts for liquid water absorption and includes an intensity offset correction, which improves the quality of the fits. A major step in the data assimilation and tropospheric air mass factor calculation has been the use of a priori profile 
shapes from the chemistry transport model TM5 at a spatial resolution of $1^{\circ} \mathrm{x} 1^{\circ}$, which together with the improved spectral fitting results in pollution hot spots being better resolved. The stratospheric estimation has also benefited from the sphericity correction that we applied in the air mass factor (AMF) calculation, which was an outcome of the radiative transfer model comparison presented in Chapter 3. We compared the QA4ECV $\mathrm{NO}_{2}$ data record to the $\mathrm{NO}_{2}$ retrieved with the DOMINO v2 algorithm and we validated it with MAX-DOAS ground based measurements. The smaller QA4ECV OMI NO 2 VCDs (5-20\% compared to DOMINO v2) show a better agreement (w.r.t. DOMINO v2) with the independent MAX-DOAS measurements over De Bilt in The Netherlands and Tai'an in China, with a mean difference of $-7 \%$ (-18\% for DOMINO v2).

In the comparison of the air mass factor calculation approaches, we identified the characteristics of the radiative transfer modelling part that could be improved and implemented those in the QA4ECV algorithm (Chapter 3). The AMF is the biggest source of uncertainty in the $\mathrm{NO}_{2}$ and $\mathrm{HCHO}$ retrieval algorithm, mainly because to represent the state of the atmosphere, several assumptions and choices have to be made. We compared the AMF calculation (step by step) of seven different retrieval groups, and we focus on characterizing the sources of structural uncertainty, which is the uncertainty that arises when different retrieval methodologies are applied to the same data.

The methodological differences in the AMF calculation start with the choice of the radiative transfer model for the TOA reflectance calculation, which only introduces an uncertainty of $2-3 \%$. The agreement in the tropospheric AMFs calculated by the different groups when using identical ancillary data (surface albedo, surface height, cloud parameters and trace gas profile shape) is of $6 \%$, uncertainty that is intrinsic to the AMF calculation and cannot be avoided. The uncertainty increases up to $42 \%$ when each group uses their preferred settings, which indicates that the assumptions and choices made to represent the state of the atmosphere introduce most of the structural uncertainty, with cloud and aerosol corrections contributing the most to it. The comparison showed that McArtim model simulates systematically lower altitude dependent air mass factors in the stratosphere as compared to DAK and we attributed these differences to McArtim's geometrically more realistic description of photon scattering in a spherical atmosphere. Based on this result, we applied a correction for sphericity when calculating the air mass factor look-up table in the QA4ECV retrieval.

The angular distribution of the light reflected by the Earth's surface influences top-ofatmosphere (TOA) reflectance values, and as a consequence satellite retrievals of albedo, clouds and $\mathrm{NO}_{2}$ are also affected. In Chapter 4 we focus on the characterization of the surface reflectance in the radiative transfer modelling for these retrievals, which usually assume that surface reflectance is isotropic. This assumption originates in the construction of the surface Lambertian Equivalent Reflector (LER) climatologies that are used to 
represent the surface albedo in cloud and trace gas retrievals. Surface LER climatologies over (under) estimate surface reflectance for forward (backward) scattering geometries, a direct consequence of the directional signature of surface reflectance that is ignored when constructing the climatologies. The under (over) estimation results in higher (lower) retrieved cloud fractions for measurements done with backward (forward) scattering geometries, and we show that this bias is removed when accounting for surface reflectance anisotropy.

To interpret the influence of surface reflectance anisotropy in cloud and $\mathrm{NO}_{2}$ retrievals, we implemented in the radiative transfer model DAK (used in the DOMINO $\mathrm{NO}_{2}$ and FRESCO and OMCDLO2 cloud retrievals) the bidirectional reflectance distribution function (BRDF) from the Ross-Li semi empirical model that fully characterizes the surface reflectance geometrical properties. This model represents the surface reflectance as a sum of three kernels that represent different types of scattering, weighted by parameters obtained from observations (e.g. MODIS). Surface BRDF effects on cloud fraction are highly relevant for scenes with low cloud fractions, where trace gas retrievals are still sensitive to pollution close to the ground. Surface BRDF effects are most relevant for cloud retrievals in the near infrared spectral range, as in the visible part of the spectrum stronger Rayleigh scattering reduces surface BRDF effects in the top of atmosphere reflectance. Surface reflectance anisotropy affects both clear-sky AMF and cloud radiance fractions, which in combination significantly affect total AMFs used in the $\mathrm{NO}_{2}$ retrievals. The combined effect, stronger than the effect of either contribution alone, is that $\mathrm{NO}_{2}$ AMFs in polluted situations increase by 25\%-30 for backward-scattering geometries and decrease by $25 \%-35 \%$ for forward-scattering geometries.

The unprecedented high resolution of TROPOMI $\mathrm{NO}_{2}$ measurements is a major improvement for atmospheric composition monitoring and brings new challenges for the retrieval community. TROPOMI $\mathrm{NO}_{2}$ retrieval algorithm inherited most of the improved aspects of the QA4ECV retrieval algorithm, and the research in Chapter 5 served as a first assessment and evaluation of the TROPOMI $\mathrm{NO}_{2}$ dataset and to test the quality and performance of the retrieval algorithm. TROPOMI measurements present an improvement over OMI, as TROPOMI clearly captures the details of pollution plumes from various sources, including cities. In Chapter 5 we explore the capabilities of TROPOMI's $\mathrm{NO}_{2}$ measurements to infer daily $\mathrm{NO}_{x}$ emissions from the megacity of Paris. We use a simple column model that focuses on the build-up of pollution over the city metropolitan area where most of the emissions occur, and together with prior knowledge of wind speed and wind direction we estimate $\mathrm{NO}_{x}$ emissions on clear-sky days. The column model method works best for days with strong wind speeds, when the loss of $\mathrm{NO}_{x}$ is dominated by advection and the prior knowledge on the chemical lifetime does not strongly influence our results. 
We applied the column model to TROPOMI $\mathrm{NO}_{2}$ data in the first half of 2018 and we compared the estimated $\mathrm{NO}_{x}$ emissions with the TNO-MACC III bottom-up emission inventory. In the winter days of February, TROPOMI estimates higher $\mathrm{NO}_{x}$ emissions than TNO-MACC inventory. In April, May and June, we find that the inventory overestimates the emission estimates, which might point to an overestimation of the residential heating by the inventories. Inventories typically assume a $18^{\circ} \mathrm{C}$ threshold for turning on the heating, and in April-June 2018 temperatures were higher for most of the clear-sky days that we analysed. In the weekends, the build up of $\mathrm{NO}_{2}$ is not as strong as on the weekdays, an indicator of weaker weekend emissions. Indeed, we estimated emissions to be up to $35 \%$ lower in weekends compared to the values during the week. Our results indicate a stronger weekend reduction compared to that predicted by the bottom-up emission inventories. The day-to-day variation captured by TROPOMI resembles the additional knowledge that we can gain by estimating emissions with TROPOMI. Bottom-up inventories use monthly, weekly and daily factors that might not reflect daily particular conditions of specific locations. For example, TROPOMI emission estimates for Fridays are generally lower than for the rest of the week, in contrast to the inventories that predict the highest emissions of the week on Fridays.

The work presented in this thesis contributes to the satellite remote sensing community (1) because of the improvement of the satellite retrieval and the knowledge of its main uncertainty sources (Chapter 2, 3 and 4), and (2) because of the application of TROPOMI $\mathrm{NO}_{2}$ measurements for the first time to infer daily $\mathrm{NO}_{x}$ emissions at urban scales (Chapter $5)$. Chapter 2 presents a community best practice $\mathrm{NO}_{2}$ retrieval algorithm, how it is has been developed in the QA4ECV project and which are the main improvements with respect to state-of-the-art retrievals. The QA4ECV retrieval algorithm has been applied to four different satellite sensors to create for the first time a coherent and harmonized data record of 20 years of $\mathrm{NO}_{2}$ (and $\mathrm{HCHO}$ ) satellite measurements. Chapter 3 presents in more detail the improvements developed in the radiative transfer part for the air mass factor calculation. Chapter 4 shows the need to coherently account for surface reflectance anisotropy in clouds and $\mathrm{NO}_{2}$ retrievals. In Chapter 5, we explore the capabilities of TROPOMI's unprecedented high resolution $\mathrm{NO}_{2}$ measurements to infer $\mathrm{NO}_{x}$ emissions from megacities. TROPOMI and future satellite sensors present new challenges for the retrieval community. The outcome of this thesis points out aspects that still need to be improved for more accurate $\mathrm{NO}_{2}$ retrievals from current and future satellite measurements. The end of this work leaves a list of to-do things for the satellite retrieval community, which will hopefully result in new scientific findings and further improvements of the satellite remote sensing capabilities. 


\section{Supplementary material}

\section{Supplementary material for Chapter 3}

Equation S3.1. Effective temperature at which the cross sections should be fitted.

$$
T_{\mathrm{eff}}=\frac{\int_{z}^{\infty} T(z) \cdot m(z) \cdot n(z) d z}{\int_{z}^{\infty} m(z) \cdot n(z) d z}
$$

Where $\mathrm{T}(\mathrm{z})$ is the temperature profile, $\mathrm{m}(\mathrm{z})$ is the altitude-dependent air mass factor, and $\mathrm{n}(\mathrm{z})$ is the $\mathrm{NO}_{2}$ number-density profile.

Equation S3.2. Temperature correction factor from Boersma et al. (2002).

$$
c_{l}=\frac{T_{0}-11.4}{T_{l}-11.4}
$$

$\mathrm{T}_{0}$ : Cross section temperature used in the DOAS fit (220 K in this study)

$\mathrm{T}_{l}$ : Temperature in layer $l$

Equation S3.3. Temperature correction factor from Bucsela et al. (2013).

$$
c_{l}=1-0.003 \cdot\left(T_{l}-T_{0}\right)
$$

$\mathrm{T}_{0}$ : Cross section temperature used in the DOAS fit (220 K in this study).

$\mathrm{T}_{l}:$ Temperature in layer $l$

Equation S3.4. Cloud radiance fraction (Boersma et al., 2004).

$$
w=\frac{f_{\mathrm{cl}} I_{\mathrm{cl}}}{f_{\mathrm{cl}} I_{\mathrm{cl}}+\left(1-f_{\mathrm{cl}}\right) I_{\mathrm{cr}}}
$$

$\mathrm{f}_{\mathrm{cl}}$ is the effective (i.e. radiometrically equivalent) cloud fraction, and $\mathrm{I}_{\mathrm{cr}}$ and $\mathrm{I}_{\mathrm{cl}}$ the fitwindow averaged radiances for $100 \%$ clear and cloudy scenes, respectively. 
Table S3.1. Model settings for top-of-the-atmosphere reflectance calculation with different RTMs, as described in Section 3.3.1.

\begin{tabular}{lll}
\hline Input parameter & $\begin{array}{l}\text { Number of } \\
\text { reference points }\end{array}$ & Values of reference points \\
\hline Wavelength & 7 & $340,360,380,400,420,440,460 \mathrm{~nm}$ \\
\hline Atmospheric profile & N.A & $\begin{array}{l}\text { Mid-latitude summer atmosphere } \\
\text { including } \mathrm{O}_{3}(335 \mathrm{DU})\end{array}$ \\
\hline$\mu_{0}($ cosine solar zenith angle) & 10 & $\begin{array}{l}1.00,0.80,0.60,0.50,0.30,0.25, \\
0.15,0.05,0.03,0.00\end{array}$ \\
\hline Solar zenith angle & 10 & $\begin{array}{l}0^{\circ}, 36.9^{\circ}, 53.1^{\circ}, 60^{\circ}, 72.5^{\circ}, \\
75.5^{\circ}, 81.4^{\circ}, 87.1^{\circ}, 88.3^{\circ}, 90^{\circ}\end{array}$ \\
\hline$\mu($ cosine viewing zenith angle $)$ & 2 & $1.00,0.30$ \\
\hline Viewing zenith angles & 2 & $0^{\circ}, 72.5^{\circ}$ \\
\hline 180 -abs $\left(\phi-\phi_{0}\right)($ relative azimuth angle $)$ & 5 & $0^{\circ}, 60^{\circ}, 90^{\circ}, 120^{\circ}, 180^{\circ}$ \\
\hline Surface albedo & 1 & 0.0 \\
\hline Surface pressure & 1 & $1013 \mathrm{hPa}$ \\
\hline
\end{tabular}

Table S3.2. Model settings for altitude dependent (box-) AMFs calculation in Section 3.3.2.

\begin{tabular}{|c|c|c|}
\hline Input parameter & $\begin{array}{l}\text { Number of } \\
\text { reference points }\end{array}$ & Values of reference points \\
\hline Atmospheric profile & N.A & $\begin{array}{l}\text { Mid-latitude summer atmosphere } \\
\text { including } \mathrm{O}_{3}(335 \mathrm{DU})\end{array}$ \\
\hline Layering & 170 & $\begin{array}{l}0,0.1,0.2, \ldots 10 \mathrm{~km} \\
10,11,12, \ldots 60 \mathrm{~km} \\
60,62,64, \ldots 100 \mathrm{~km}\end{array}$ \\
\hline$\mu_{0}($ cosine solar zenith angle $)$ & 12 & $\begin{array}{l}1.00,0.90,0.80,0.70,0.60,0.50, \\
0.30,0.250 .15,0.05,0.03,0.00\end{array}$ \\
\hline Solar zenith angle & 10 & $\begin{array}{l}0^{\circ}, 25.8^{\circ}, 36.9^{\circ}, 45.6^{\circ}, 53.1^{\circ}, 60^{\circ}, 72.5^{\circ} \\
75.5^{\circ}, 81.4^{\circ}, 87.1^{\circ}, 88.3^{\circ}, 90^{\circ}\end{array}$ \\
\hline$\mu$ (cosine viewing zenith angle) & 6 & $1.00,0.90,0.80,0.70,0.50,0.30$ \\
\hline Viewing zenith angles & 6 & $0^{\circ}, 25.8^{\circ}, 36.9^{\circ}, 45.6^{\circ}, 60^{\circ}, 72.5^{\circ}$ \\
\hline $180-\operatorname{abs}\left(\phi-\phi_{0}\right)($ relative azimuth angle) & 13 & $\begin{array}{l}0^{\circ}, 15^{\circ}, 30^{\circ}, 45^{\circ}, 60^{\circ}, 75^{\circ}, 90^{\circ}, \\
135^{\circ}, 150^{\circ}, 165^{\circ}, 180^{\circ}\end{array}$ \\
\hline Surface albedo & 7 & $0.00,0.05,0.1,0.2,0.5,0.8,1.0$ \\
\hline Surface pressure $(\mathrm{hPa})$ & 5 & $1013,902,802,554,281$ \\
\hline
\end{tabular}

\section{S 3.1 Preferred settings for $\mathrm{NO}_{2}$ tropospheric AMF calculation}

S 3.1.1 BIRA-IASB. For the radiative transfer modelling and box-AMF calculation, BIRA uses the VLIDORT radiative transfer model (see Sect. 3.2.2). The surface reflectivity is a combination of the MODIS black sky albedo (BSA) gap filled product (MCD43GF) and the OMI minimum LER from Kleipool et al. (2008) at $440 \mathrm{~nm}$. The MODIS BSA values are averaged over 10 years of measurements and the OMI min LER dataset is used to fill the gaps and for scenes over water. Surface pressure is from the Global Multi-resolution Terrain Elevation Data 2010 with $30 \times 30 \mathrm{~km}$ resolution, corrected following the approach by Zhou et al. (2009). The cloud parameters (cloud fraction and cloud pressure) are taken from the OMI 02-02 cloud retrieval (OMCLDO2, Acarreta et al. (2004)). For the cloud correction they apply IPA for cloud fractions higher than 0.2 and cloud masking for cloud fractions lower than 0.2. They apply an 

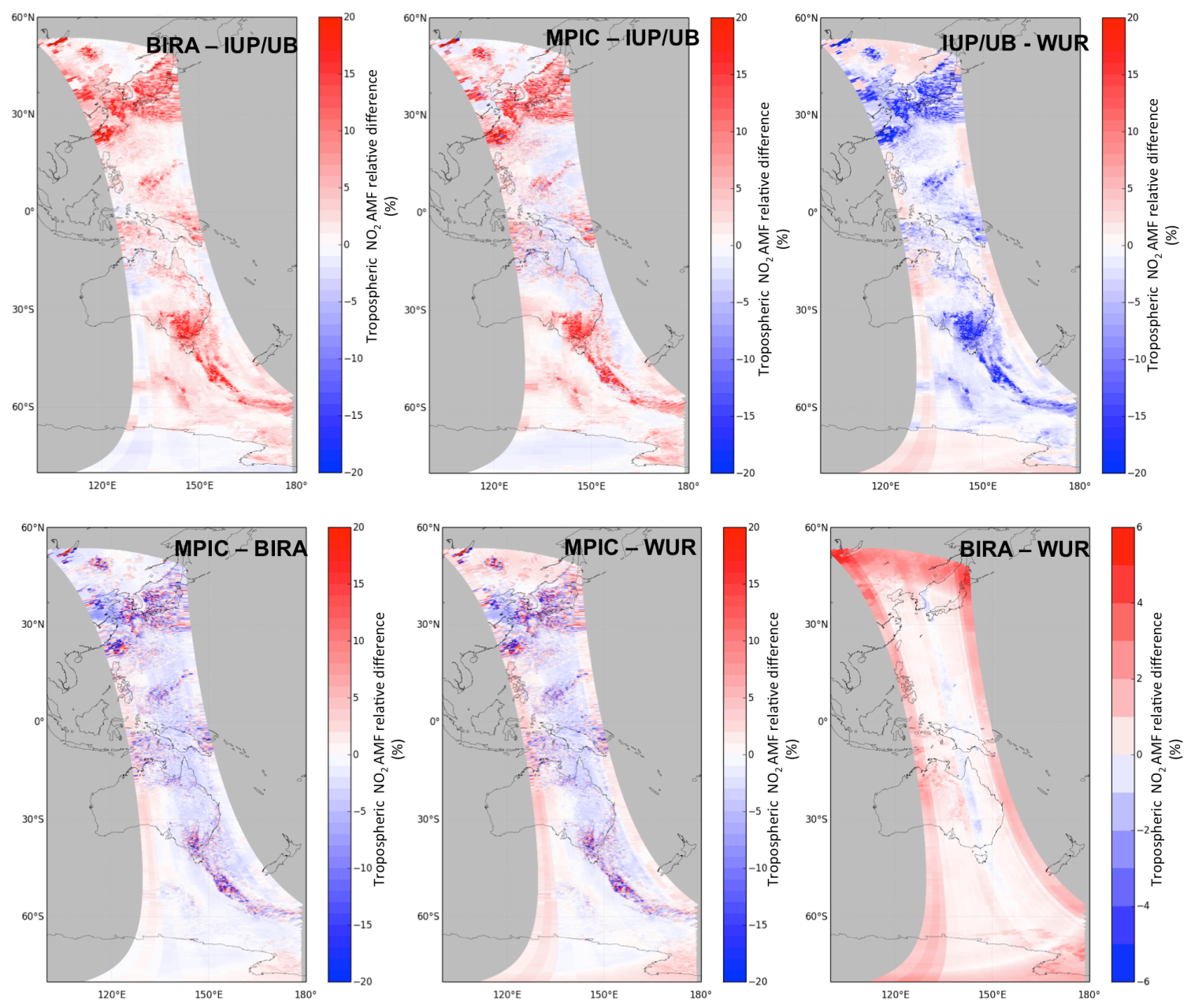

Figure S3.1: Relative differences of tropospheric $\mathrm{NO}_{2}$ AMFs between each research group using harmonized settings. Only pixels with SZA $<70^{\circ}$ are shown. The selected OMI orbit is from 02 February 2005 (2005m0202-o02940 v003). Different scale was used for the differences between BIRA and WUR (lower right panel).

implicit aerosol correction. The $\mathrm{NO}_{2}$ a priori profiles are daily profiles from the TM5 chemistry transport model at a resolution of $1 \mathrm{x} 1$ degrees.

S 3.1.2 IUP-UB. For the radiative transfer modelling and box-AMF calculation, IUP-UB uses the SCIATRAN radiative transfer model (see Sect. 3.2.2). Surface albedo is from Kleipool et al. (2008) version 3, which uses 5 years of OMI measurements. The monthly minimum LER at $442 \mathrm{~nm}$ is used. Surface pressure is from the Global Multi-resolution Terrain Elevation Data 2010. They are gridded to $0.25 \times 0.25 \mathrm{deg}$. and corrected following the approach by Acarreta et al. (2004). For the cloud correction they apply independent pixel approximation (IPA) for cloud fractions higher than 0.1 and cloud masking for cloud fractions lower than 0.1. They use modelled reflectances for the current albedo and a cloud albedo of 0.8 to convert O2-O2 cloud fraction to radiance fraction. The cloud fraction threshold is cloud radiance fraction of $50 \%$. 


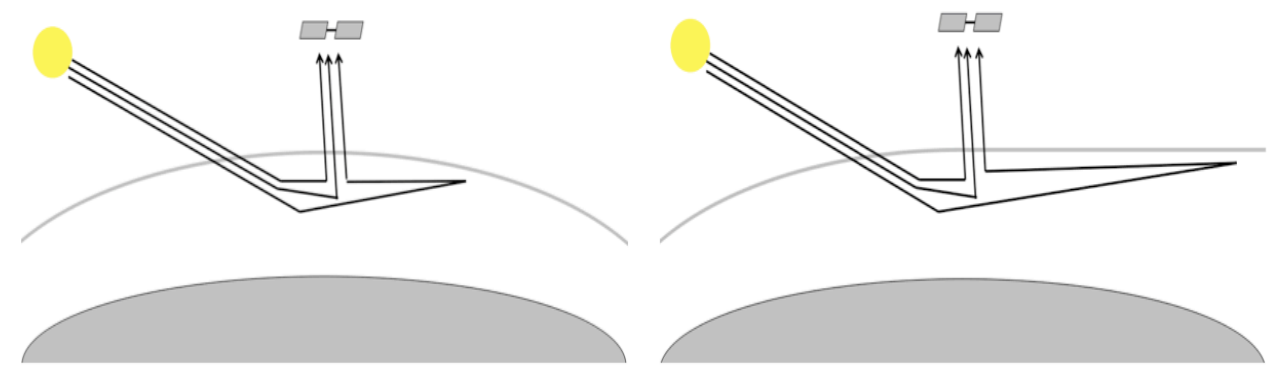

Figure S3.2: Schematic representation of differences in model design between McArtim (left) and DAK, VLIDORT and SCIATRAN (right) for the direct solar beam (left side of the individual figures) and the multiple scattered photons (left side of the individual figures). The grey line indicates the atmosphere's confinement (either spherical or plane parallel).

They apply an implicit aerosol correction. The $\mathrm{NO}_{2}$ and temperature profiles come are daily MACC-II reanalysis profiles with a resolution of $1.25^{\circ} \times 1.25^{\circ}$.

S 3.1.3 MPI-C . For the radiative transfer modelling and box-AMF calculation, MPI-C uses the McArtim radiative transfer model (see Sect. 3.2.2). Surface albedo is from Kleipool et al. (2008), version 002, which uses 3.5 years of OMI measurements. The monthly minimum LER at $440 \mathrm{~nm}$ is used. Surface pressure is from TM4 chemistry transport model and corrected following Zhou et al. (2009) approach using the high resolution DEM 3km Earth Science Data type database. The $\mathrm{NO}_{2}$ and temperature profiles are daily TM4 model at a resolution of $3 \mathrm{x}$ 2 deg. In the preferred settings, MPI-C accounts for possible cloud aerosol mixtures or layer of other different types of aerosol. They differentiate three cases:

(A) Clouds higher than $3 \mathrm{~km}$. The IPA is applied to calculate the AMF.

(B) Low clouds and aerosols. For clouds below $2 \mathrm{~km}$, a parametrized aerosol-cloud layer is included between 0 and $1 \mathrm{~km}$ above the surface. This parametrization represents a coarse cloud/aerosol model that assumes small cloud fractions to be pure aerosols and high cloud fractions to be pure clouds both with a fixed layer thickness of $1 \mathrm{~km}$. They determine the relation between optical depth of an aerosol/cloud layer and the cloud radiance fraction using McArtim. For this purpose they expand the LUT by the optical depth (OD), single scattering albedo and the Henyey Greenstein asymmetry parameter. Depending on the optical depth, they assume typical optical parameters of aerosols for OD $\leq 1$, aerosols/cloud particle mixture for $1<$ OD $<3$ and cloud particles for OD $>3$.

(C) Low cloud fraction. For clouds between 2 and $3 \mathrm{~km}$ and cloud radiance fraction below $10 \%$, they use the clear sky AMF.

(D) High cloud fraction. For clouds between 2 and $3 \mathrm{~km}$ and cloud radiance fraction higher than $10 \%$, the pixel is invalid as it cannot be differentiated between white Lambertian clouds and mixtures of clouds and aerosols. 

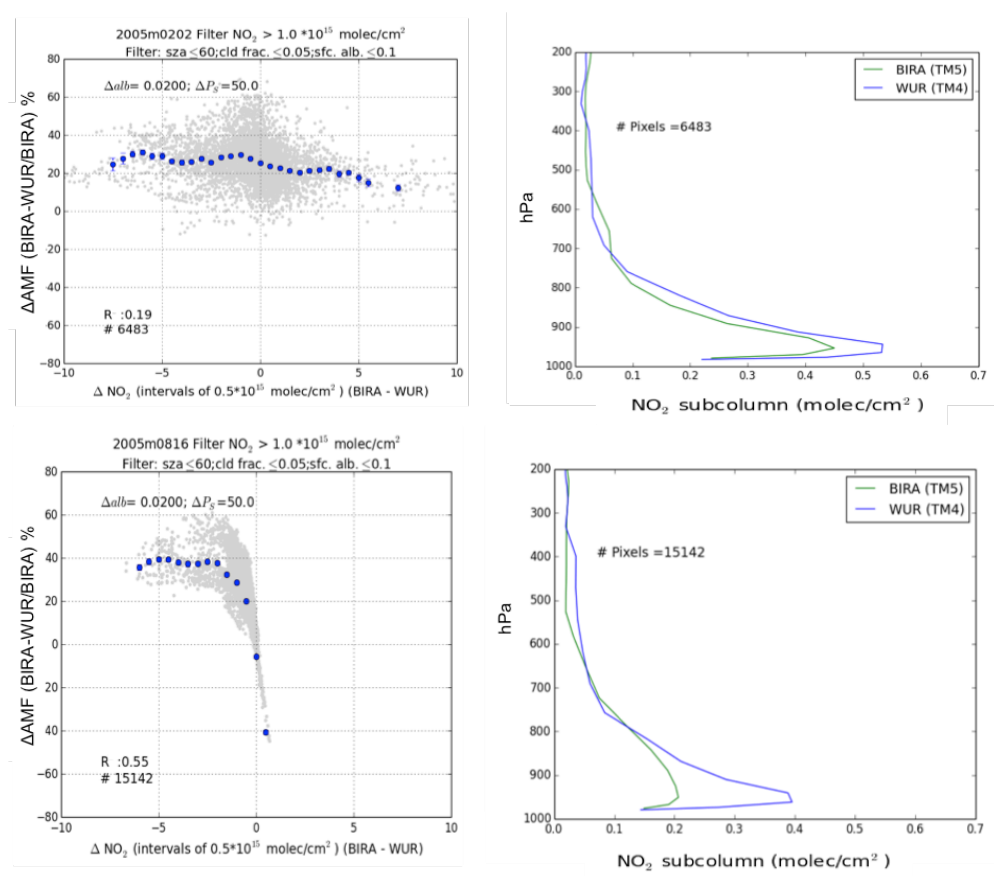

Figure S3.3: Example of correlation between AMF differences by BIRA and WUR ( $\triangle \mathrm{AMF}$ ) and differences in $\mathrm{NO}_{2}$ vertical columns $\left(\Delta \mathrm{NO}_{2}\right)$ for 02 February 2005 (upper panels) and 16 August 2005 (lower panels). The panels on the right show the average $\mathrm{NO}_{2}$ vertical profiles for the scenarios shown in the left panels (green, TM5 by BIRA and blue, TM4 by WUR).

Table S3.3. Number (\#) of pixels and correlation coefficient $(\mathrm{R})$ for the correlation between air mass factor differences between WUR and BIRA $(\triangle \mathrm{AMF})$ with differences in modelled $\mathrm{NO}_{2}$ vertical column $\left(\Delta \mathrm{NO}_{2}\right)$, surface albedo $\left(\Delta \mathrm{A}_{\mathrm{s}}\right)$ and surface pressure $\left(\Delta \mathrm{P}_{\mathrm{s}}\right)$ for 02 February 2005 (upper panels) and 16 August 2005 (lower panels). The first column corresponds to the correlation shown in left panels in Fig. S3.3.

\begin{tabular}{llll}
\hline 2005m0202 & $\Delta$ AMF vs. $\Delta \mathrm{NO}_{2}$ & $\Delta$ AMF vs. $\Delta \mathrm{A}_{\mathrm{s}}$ & $\Delta$ AMF vs. $\Delta \mathrm{P}_{\mathrm{s}}$ \\
\hline \# Pixels & 6843 & 1876 & 1303 \\
\hline $\mathbf{R}$ & -0.19 & 0.50 & -0.04 \\
\hline 2005m0816 & $\Delta$ AMF vs. $\Delta \mathrm{NO}_{2}$ & $\Delta$ AMF vs. $\Delta \mathrm{A}_{\mathrm{s}}$ & $\Delta$ AMF vs. $\Delta \mathrm{P}_{\mathrm{s}}$ \\
\hline \# Pixels & 15142 & 5382 & 2736 \\
\hline $\mathbf{R}$ & -0.55 & 0.21 & -0.01 \\
\hline
\end{tabular}


Table S3.4. Number (\#) of pixels and correlation coefficient (R) for the correlation between air mass factor differences between Peking Uni. and WUR ( $\triangle \mathrm{AMF})$ with differences in cloud pressure $\left(\Delta \mathrm{P}_{\mathrm{c}}\right)$ and modelled $\mathrm{NO}_{2}$ vertical column $\left(\Delta \mathrm{NO}_{2}\right)$ for 02 February 2005 and 16 August 2005.

\begin{tabular}{lll}
\hline 2005m0202 & $\Delta$ AMF vs. $\Delta \mathrm{P}_{c}$ & $\Delta$ AMF vs. $\Delta \mathrm{NO}_{2}$ \\
\hline \# Pixels & 397 & 981 \\
\hline $\mathbf{R}$ & 0.28 & 0.151 \\
\hline 2005m0816 & $\Delta$ AMF vs. $\Delta \mathrm{P}_{c}$ & $\Delta$ AMF vs. $\Delta \mathrm{NO}_{2}$ \\
\hline \# Pixels & 576 & 310 \\
\hline $\mathbf{R}$ & -0.12 & 0.17 \\
\hline
\end{tabular}

Table S3.5. Tropospheric $\mathrm{NO}_{2}$ AMFs calculated by Peking Uni. with and without an explicit aerosol correction over China on 02 February 2005. Pixels with AOT $>0.5$, albedo $<0.3$ and effective cloud fraction $<0.5$ were selected. The average AOT and single scattering albedo originate from the GEOS-Chem aerosol simulations for the location and time of the pixels. The average cloud fraction and cloud pressure are the result from Peking Uni. cloud retrieval.

\begin{tabular}{|c|c|c|c|c|}
\hline & \multicolumn{2}{|c|}{$\mathrm{AMF}_{\text {aer }}<\mathrm{AMF}$} & \multicolumn{2}{|c|}{$\mathrm{AMF}_{\text {aer }}>\mathrm{AMF}$} \\
\hline \# Pixels & \multicolumn{2}{|c|}{441} & \multicolumn{2}{|c|}{149} \\
\hline AOT & \multicolumn{2}{|c|}{1.1} & \multicolumn{2}{|c|}{0.7} \\
\hline SSA & \multicolumn{2}{|c|}{0.90} & \multicolumn{2}{|c|}{0.88} \\
\hline & $\begin{array}{l}\text { Without } \\
\text { correction }\end{array}$ & $\begin{array}{l}\text { With } \\
\text { correction }\end{array}$ & $\begin{array}{l}\text { Without } \\
\text { correction }\end{array}$ & $\begin{array}{l}\text { With } \\
\text { correction }\end{array}$ \\
\hline Cloud fraction & 0.18 & 0.15 & 0.31 & 0.19 \\
\hline Cloud Pressure & $791 \mathrm{hPa}$ & $432 \mathrm{hPa}$ & $689 \mathrm{hPa}$ & $666 \mathrm{hPa}$ \\
\hline AMF & 1.78 & 0.80 & 0.53 & 0.94 \\
\hline
\end{tabular}
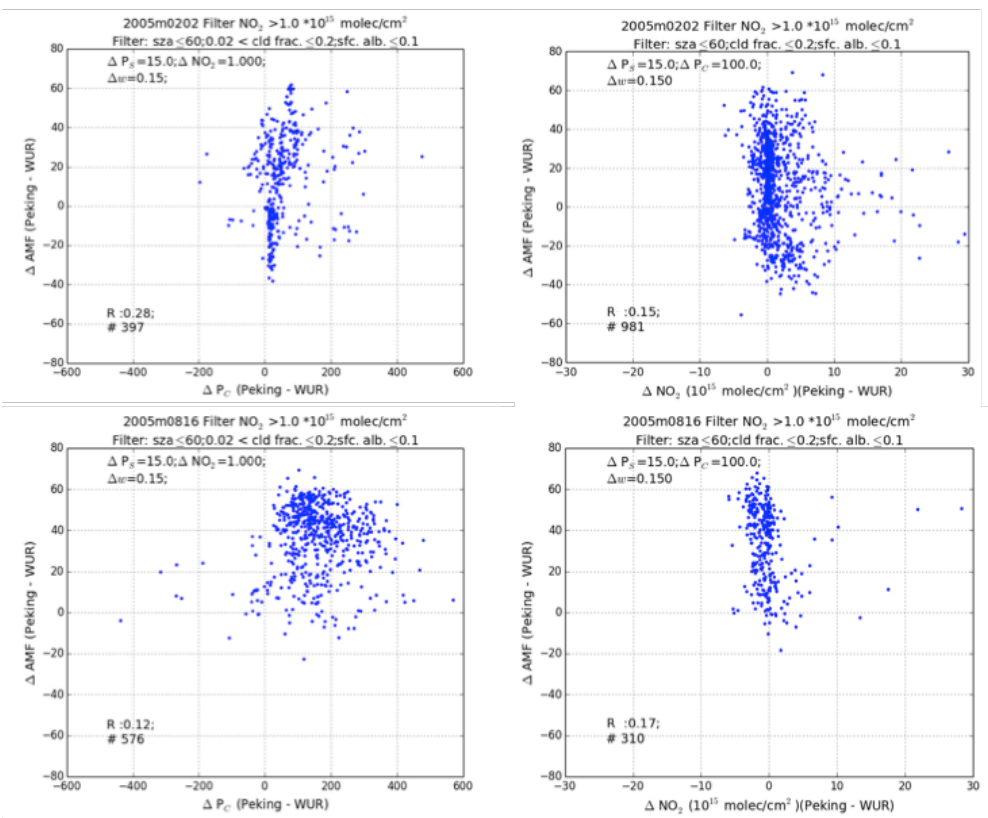

Figure S3.4: Correlation between AMF differences by Peking University and WUR $(\triangle \mathrm{AMF})$ and differences in cloud pressure $\left(\Delta \mathrm{P}_{\mathrm{c}}\right)$ and $\mathrm{NO}_{2}$ vertical columns $\left(\Delta \mathrm{NO}_{2}\right)$ for the 02 February 2005 (upper panels) and 16 August 2005 (lower panels). 

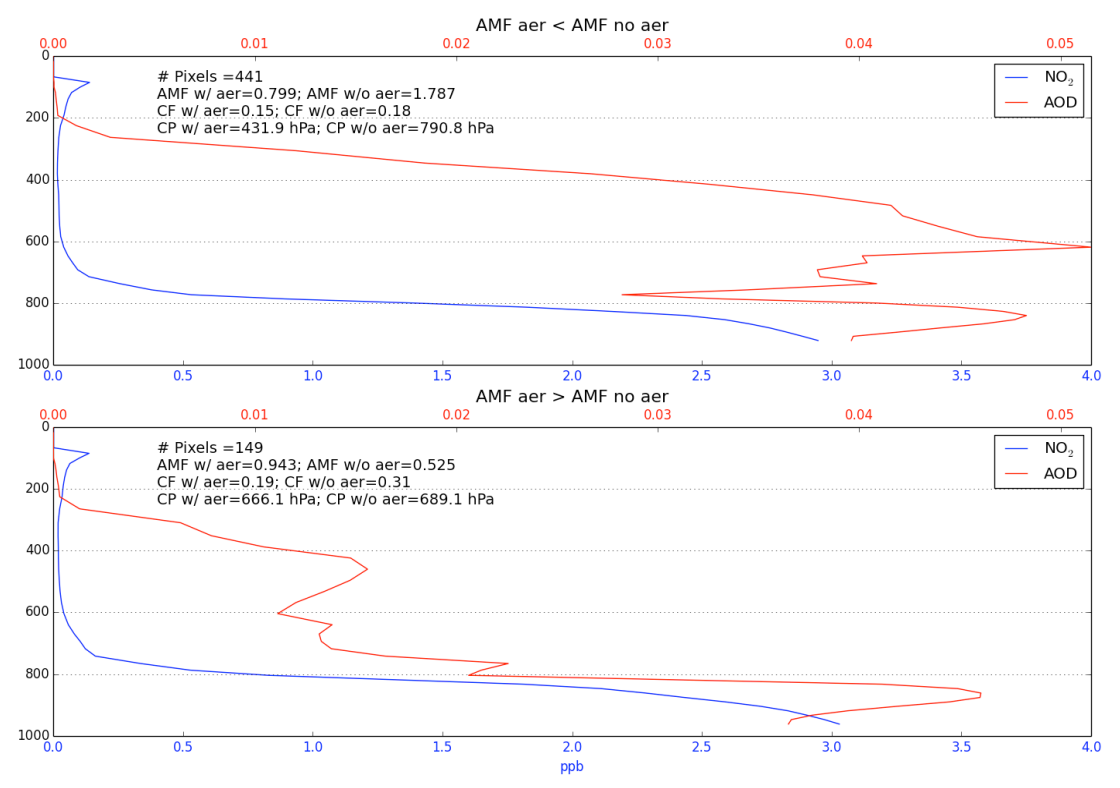

Figure S3.5: Aerosol optical depth (red line) and a priori $\mathrm{NO}_{2}$ (blue line) vertical profiles for 02 February 2005. Upper panel: pixels where $\mathrm{AMF}_{\text {aer }}$ (with explicit aerosol correction) are lower than AMF (without explicit aerosol correction), due to the screening effect of the aerosols layer above the $\mathrm{NO}_{2}$ layer. Lower panel: pixels where $\mathrm{AMF}_{\text {aer }}$ (with explicit aerosol correction) are higher than AMF (without explicit aerosol correction), due to the increased scattering probability within the $\mathrm{NO}_{2}+$ aerosol layer. Only pixels with AMF relative differences higher than $25 \%$ are shown, as well as surface reflectance $<0.3$, effective cloud fraction $<0.5$ and $\mathrm{AOD}>0.5$.
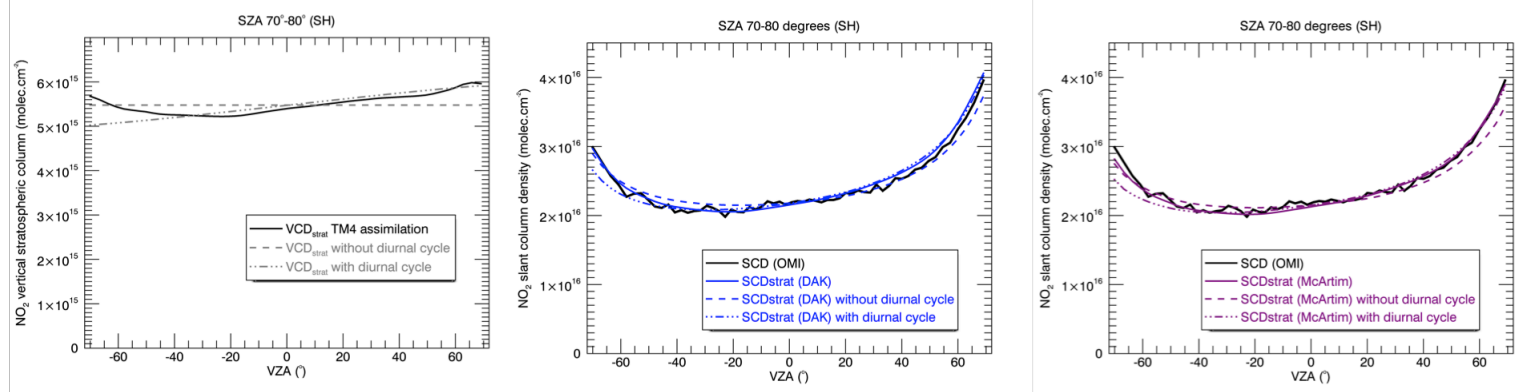

Figure S3.6: (Left panel) Stratospheric vertical $\mathrm{NO}_{2}$ columns as a function of VZA from assimilation of OMI $\mathrm{NO}_{2}$ SCDs in TM4 (DOMINO V2 product). Dashed line: scenario without any diurnal variation in stratospheric $\mathrm{NO}_{2}$ (local solar time differences are up to 6 hours at these latitudes), dashed-dotted line: scenario with a strong, consistent stratospheric $\mathrm{NO}_{2}$ increase rate (of $0.15 \cdot 10^{15} \mathrm{molec} / \mathrm{cm}^{2} / \mathrm{h}$ ). Central and right panel compare the three corresponding simulated stratospheric slant columns (from TM4 assimilated VCD, without diurnal cycle and with diurnal cycle) from DAK (left panel) and McArtim (right panel) to the observed OMI total SCD (black solid line) as a function of OMI VZA. 
Table S3.6. Statistical parameters of the comparison with the model mean (in \% (100(ab)/a)) of total tropospheric $\mathrm{NO}_{2}$ AMFs calculated by each group over the globe for polluted and unpolluted pixels (pixels with model $\mathrm{NO}_{2}$ vertical column higher or lower than $1 \cdot 10^{15}$ molec $/ \mathrm{cm}^{2}$ respectively). Upper panels correspond to OMI measurements for the 02 February 2005 and the lower panels for the 16 August 2005. Only pixels with cloud fraction $\leq 0.2$ and $\mathrm{SZA}<60^{\circ}$ are considered in the comparison.

\begin{tabular}{|c|c|c|c|c|c|c|}
\hline \multicolumn{7}{|c|}{ Polluted pixels } \\
\hline & Mean & Median & $\sigma$ & $\mathrm{R}^{2}$ & Slope & Offset \\
\hline BIRA & -14.0 & -15.8 & 15.6 & 0.840 & 1.40 & -0.33 \\
\hline IUP-UB & 2.0 & 2.0 & 20.2 & 0.606 & 1.10 & -0.17 \\
\hline Leicester Uni. & 3.7 & 3.8 & 14.6 & 0.788 & 1.07 & -0.14 \\
\hline MPIC & -8.0 & 7.1 & 42.1 & 0.699 & 2.60 & -1.85 \\
\hline NASA & -1.6 & -1.2 & 11.7 & 0.847 & 1.05 & -0.05 \\
\hline WUR & 18.0 & 18 & 12.5 & 0.814 & 1.06 & -0.30 \\
\hline \multicolumn{7}{|c|}{ Unpolluted pixels } \\
\hline BIRA & -5.9 & -6.1 & 10.4 & 0.897 & 1.08 & -0.05 \\
\hline IUP-UB & 1.7 & 1.2 & 11.6 & 0.861 & 1.05 & -0.12 \\
\hline Leicester Uni. & 7.4 & 7.7 & 8.4 & 0.926 & 1.04 & -0.2 \\
\hline MPIC & -2.6 & -2.4 & 17.9 & 0.827 & 1.45 & -0.75 \\
\hline NASA & -2.5 & -1.5 & 9.5 & 0.940 & 1.16 & -0.31 \\
\hline WUR & 1.8 & 1.5 & 8.8 & 0.938 & 1.13 & -0.26 \\
\hline \multicolumn{7}{|c|}{ Polluted pixels } \\
\hline & Mean & Median & $\sigma$ & $\mathrm{R}^{2}$ & Slope & Offset \\
\hline BIRA & -9.7 & -13.2 & 15.5 & 0.916 & 1.39 & -0.36 \\
\hline IUP-UB & -7.3 & -6.2 & 15.3 & 0.859 & 1.04 & 0.02 \\
\hline Leicester Uni. & 1.3 & 1.9 & 10.6 & 0.921 & 0.97 & 0.01 \\
\hline MPIC & 1.8 & 10.2 & 31.1 & 0.643 & 1.54 & -0.71 \\
\hline NASA & -1.7 & -1.5 & 11.5 & 0.918 & 1.08 & -0.08 \\
\hline WUR & 15.7 & 13.9 & 10.3 & 0.926 & 1.03 & -0.23 \\
\hline \multicolumn{7}{|c|}{ Unpolluted pixels } \\
\hline BIRA & -5.2 & -5.9 & 9.3 & 0.929 & 1.1 & -0.09 \\
\hline IUP-UB & $-0.8-$ & 0.7 & 11.3 & 0.875 & 1.01 & -0.01 \\
\hline Leicester Uni. & 6.0 & 6.7 & 8.5 & 0.923 & 0.99 & -0.1 \\
\hline MPIC & -2.0 & -1.4 & 15.9 & 0.871 & 1.36 & -0.61 \\
\hline NASA & -2.5 & -1.5 & 9.5 & 0.940 & 1.16 & -0.31 \\
\hline WUR & 3.7 & 3.6 & 9.3 & 0.932 & 1.13 & -0.29 \\
\hline
\end{tabular}


Table S3.7. Statistical parameters of the comparison with the model mean (in \% (100(ab)/a)) of total tropospheric $\mathrm{NO}_{2}$ AMFs calculated by each group over China $\left(20^{\circ}-53^{\circ} \mathrm{N} /\right.$ $80^{\circ}-130^{\circ} \mathrm{W}$ ) for polluted and unpolluted pixels (pixels with model $\mathrm{NO}_{2}$ vertical column higher or lower than $1 \cdot 10^{15} \mathrm{molec} / \mathrm{cm}^{2}$ respectively). Upper panels correspond to OMI measurements for the 02 February 2005 and the lower panels for the 16 August 2005. Only pixels with cloud fraction $\leq 0.2$ and $\mathrm{SZA}<60^{\circ}$ are considered in the comparison.

\begin{tabular}{|c|c|c|c|c|c|c|}
\hline \multicolumn{7}{|c|}{ Polluted pixels } \\
\hline & Mean & Median & $\sigma$ & $\mathrm{R}^{2}$ & Slope & Offset \\
\hline BIRA & -10 & -7.5 & 18.2 & 0.769 & 1.42 & -0.37 \\
\hline IUP-UB & 5.1 & 9.0 & 14.9 & 0.728 & 0.70 & 0.27 \\
\hline Leicester Uni. & -8.8 & -3.3 & 20.4 & 0.649 & 0.96 & 0.13 \\
\hline MPIC & 7.1 & 8.2 & 37.1 & 0.781 & 2.46 & -1.72 \\
\hline NASA & -0.2 & 1.5 & 11.9 & 0.843 & 0.94 & 0.06 \\
\hline Peking Uni. & -3.3 & -4.8 & 18.0 & 0.774 & 1.27 & -0.28 \\
\hline WUR & 10.79 .9 & 13.0 & 0.880 & 1.22 & -0.37 & \\
\hline \multicolumn{7}{|c|}{ Unpolluted pixels } \\
\hline BIRA & -15 & -15.1 & 15.2 & 0.860 & 1.27 & -0.18 \\
\hline IUP-UB & 8.3 & 12.6 & 16.2 & 0.745 & 1.08 & -0.24 \\
\hline Leicester Uni. & 1.2 & 2.1 & 10.2 & 0.905 & 0.98 & 0.01 \\
\hline MPIC & $-5.1-3.2$ & 27.5 & 0.728 & 1.64 & -0.88 & \\
\hline NASA & $-2.5-2.9$ & 11.4 & 0.910 & 1.13 & -0.15 & \\
\hline Peking Uni. & 2.9 & 3.9 & 20.7 & 0.762 & 1.33 & -0.54 \\
\hline WUR & 10.1 & 10.2 & 12.3 & 0.882 & 1.11 & -0.31 \\
\hline \multicolumn{7}{|c|}{ Polluted pixels } \\
\hline & Mean & Median & $\sigma$ & $\mathrm{R}^{2}$ & Slope & Offset \\
\hline BIRA & -10.5 & -10.9 & 13.4 & 0.855 & 1.41 & -0.33 \\
\hline IUP-UB & -20.0 & -20.8 & 14.0 & 0.767 & 1.13 & 0.06 \\
\hline Leicester Uni. & 7.2 & 8.1 & 9.7 & 0.871 & 1.06 & -0.14 \\
\hline MPIC & 26.0 & 27.0 & 12.7 & 0.708 & 1.06 & -0.34 \\
\hline NASA & -0.9 & 0.7 & 17 & 0.778 & 1.43 & -0.46 \\
\hline Peking Uni. & -24.6 & -25.3 & 20.0 & 0.775 & 1.81 & -0.60 \\
\hline WUR & 22.8 & 23.3 & 12.5 & 0.701 & 1.04 & -0.29 \\
\hline \multicolumn{7}{|c|}{ Unpolluted pixels } \\
\hline BIRA & -10.3 & -10.2 & 8.6 & 0.960 & 1.27 & -0.26 \\
\hline IUP-UB & -0.9 & 0.1 & 9.6 & 0.899 & 0.87 & 0.20 \\
\hline Leicester Uni. & 8.0 & 7.6 & 7.8 & 0.931 & 0.97 & -0.09 \\
\hline MPIC & -1.0 & -0.9 & 10.6 & 0.929 & 1.27 & -0.40 \\
\hline NASA & 4.7 & 4.3 & 11.2 & 0.874 & 1.05 & -0.16 \\
\hline Peking Uni. & -3.2 & -0.9 & 15.3 & 0.822 & 1.18 & -0.24 \\
\hline WUR & 2.8 & 3.3 & 8.6 & 0.934 & 1.10 & -0.19 \\
\hline
\end{tabular}




\section{Supplementary material for Chapter 4}

\section{S4.1 Kernels for the Ross-Li BRDF model}

Expressions of the kernels implemented in DAK to model the surface reflectance anisotropy. We refer to the original literature where these kernels were derived for more details.

Ross-Thick kernel. The expression of the Ross-Thick volumetric scattering kernel is (Roujean et al., 1992):

$$
K_{\mathrm{vol}}=\left[\frac{(\pi / 2-\xi) \cos \xi+\sin \xi}{\cos \theta^{\prime}+\cos \theta}\right] \cdot\left[1+\left(1+\xi / \xi_{0}\right)^{-1}\right]-\frac{\pi}{4} .
$$

Here $\theta^{\prime}$ and $\theta$ are the incident and reflected zenith angles, respectively. $\xi$ is the scattering angle defined as:

$$
\cos \xi=\cos \theta \cos \theta^{\prime}+\sin \theta \sin \theta^{\prime} \cos \left(\varphi-\varphi^{\prime}\right)
$$

where $\varphi-\varphi^{\prime}$ is the relative azimuth angle (reflected and incident azimuth difference).

The term in the second pair of squared bracket in Eq. A1 is the modified part for the hotspot modelling, where $\xi_{0}$ is the hot spot characteristic angle (typically $1.5^{\circ}$ ). This characteristic angle can be related to the ratio of the size of the scattering element and the canopy vertical density (Maignan et al., 2004).

Li-Sparse kernel. The expression of the Li-Sparse geometric scattering kernel is ( $\mathrm{Li}$ and Strahler (1986), Wanner et al. (1995)):

$$
\begin{gathered}
K_{\mathrm{geo}}=O\left(\theta, \theta^{\prime}, \varphi-\varphi^{\prime}\right)-\sec \theta^{*}-\sec \theta^{\prime *}+\frac{1}{2}\left(1+\cos \xi^{*}\right) \sec \theta^{*} \sec \theta^{\prime *} \\
O=\frac{1}{\pi}(t-\sin t \cos t)\left(\sec \theta^{*}+\sec \theta^{\prime *}\right), \\
\cos t=\frac{h}{b} \frac{\sqrt{D^{2}+\left(\tan \theta^{\prime *} \tan \theta^{*} \sin \left(\varphi-\varphi^{\prime}\right)\right)^{2}}}{\sec \theta^{*} \sec \theta^{\prime *}}, D=\sqrt{\tan ^{2} \theta^{*}+\tan ^{2} \theta^{\prime *}+2 \tan \theta^{*} \tan \theta^{\prime *} \cos \left(\varphi-\varphi^{\prime}\right) .}
\end{gathered}
$$

The angles with a star are equivalent angles to convert spheroid-like object to spheres:

$$
\theta^{*}=\tan ^{-1}\left(\frac{b}{r} \tan \theta\right), \quad \theta^{* *}=\tan ^{-1}\left(\frac{b}{r} \tan \theta^{\prime}\right)
$$

$O$ (Eq. S4.4) is the overlap area between the shadow of illumination and the shadow of viewing projections on the ground. $D$ is the distance between the centers of the scattering objects. The parameter $t$ is used to parametrize the scattering objects spherically. The kernel 
it is not linear as it has two parameters $\frac{b}{r}$ and $\frac{h}{b}$ describing the shape and the relative height of the scattering objects, in this study are set to 1 and 2 respectively. 


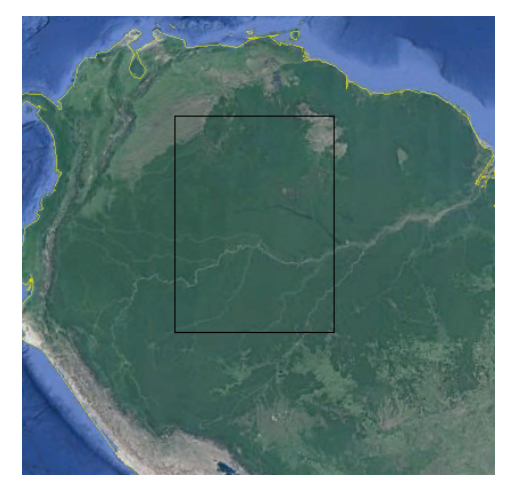

Figure S4.1: Google Earth image of the Amazon region (Lat.: 5N-10S and Lon.: 70W-60W) analysed in the manuscript.
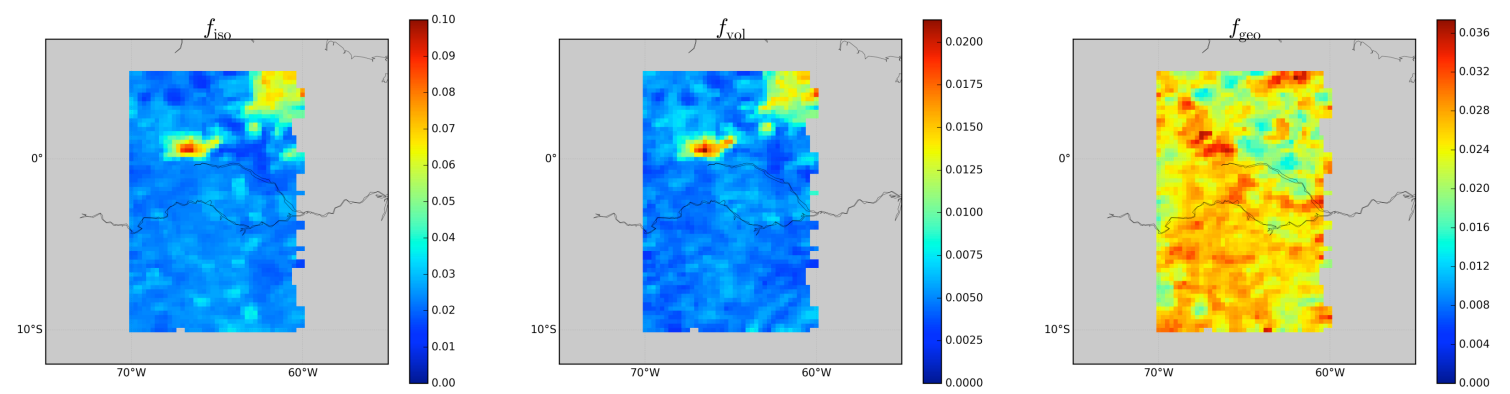

Figure S4.2: Monthly averaged BRDF parameters $\left(f_{\text {iso }}, f_{\text {vol }}, f_{\text {geo }}\right)$ from MODIS Band 3 (459$479 \mathrm{~nm}$ ) averaged over the Amazon in a $0.25^{\circ} \mathrm{x} 0.25^{\circ}$ grid for March. BRDF parameters are from a 16-year climatology (2000-2016) created by the QA4ECV land group.
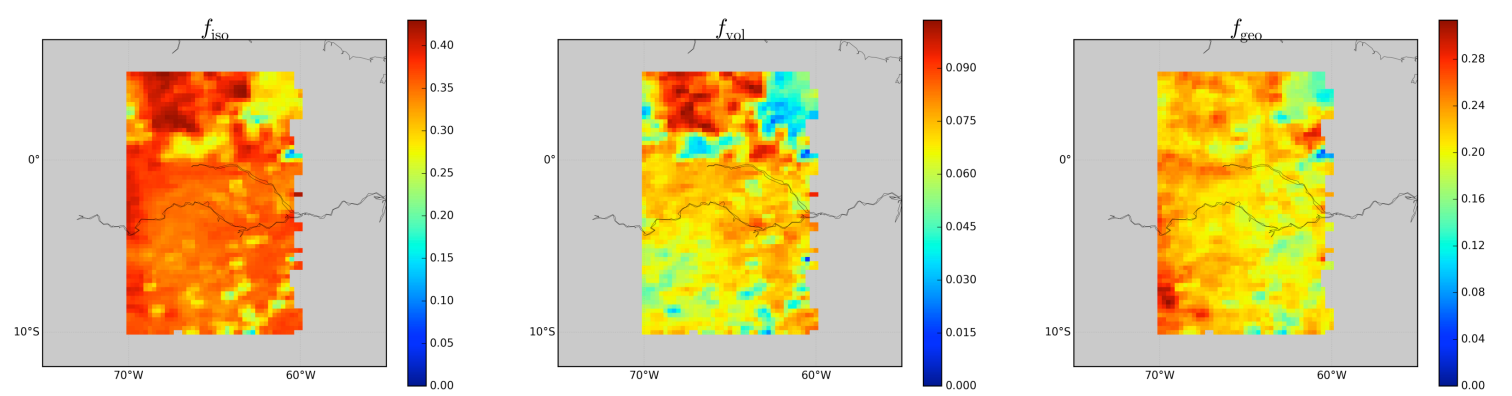

Figure S4.3: Same as Fig. S4.2 but for Band2 (841 - $876 \mathrm{~nm})$. 


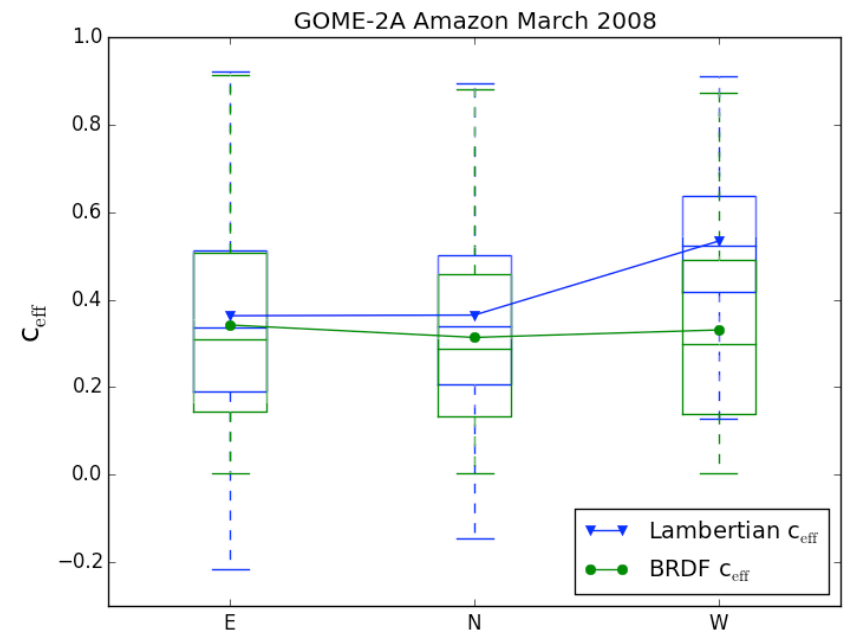

Figure S4.4: Box-plot of simulated cloud fractions with a Lambertian surface (blue) and with surface BRDF (green) for GOME-2A East, Nadir and West measurements over Amazonia for March 2008. Triangles and circles show the mean values and the box represents 25 th and 75 th percentiles and the dashed lines the minimum and maximum values.
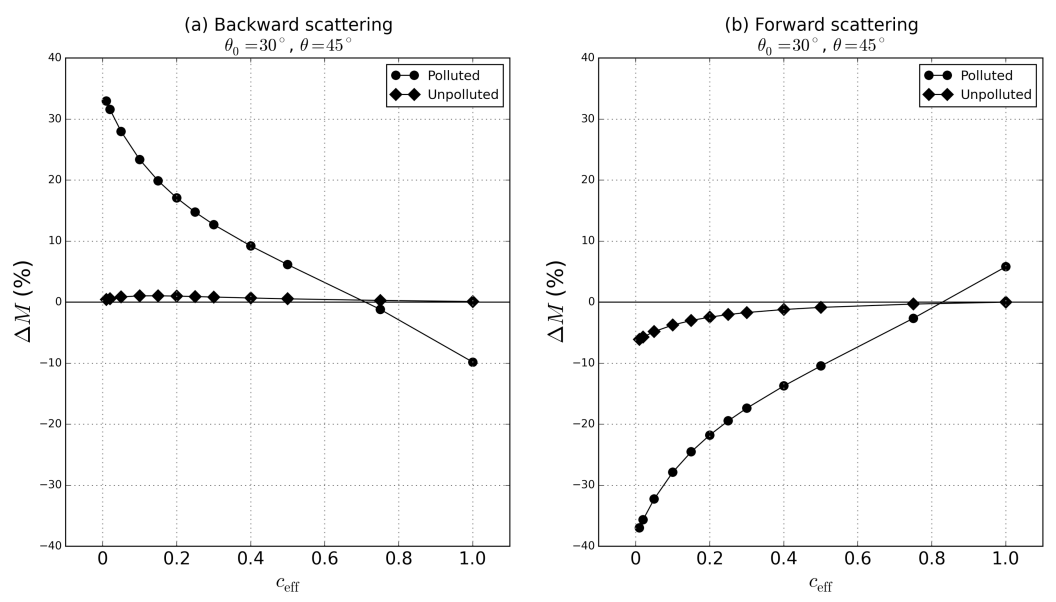

Figure S4.5: Relative differences between total tropospheric $\mathrm{NO}_{2}$ AMF computed with surface BRDF and with a Lambertian surface as a function of cloud fraction for (a) backward scattering direction and (b) forward scattering direction for $\left(\theta, \theta_{0}\right)=\left(30^{\circ}, 45^{\circ}\right)$ for a moderately polluted (circles) and unpolluted (squares) troposphere. Surface BRDF parameters are $\left(f_{\text {iso }}, f_{\text {vol }}, f_{\text {geo }}\right)=(0.04,0.03,0.008)$ and $A_{\mathrm{ws}}=0.036$ for the Lambertian surface. 


\section{Supplementary material for Chapter 5}

\section{S5.1 Validation of TROPOMI $\mathrm{NO}_{2}$ over Paris}

To evaluate the quality of TROPOMI $\mathrm{NO}_{2}$ data over Paris, we generated a set of independent $\mathrm{NO}_{2}$ columns based on coincident measurements of $\mathrm{NO}_{2}$ concentrations measured at the Eiffel Tower at $300 \mathrm{~m}$ altitude, and knowledge of the boundary layer height. The hourly $\mathrm{NO}_{2}$ concentration values closest in time to the TROPOMI overpass were converted (multiplied by 1.4, motivated by Figure 1b in Dieudonné et al. (2013) into surface concentrations $\mathrm{C}_{g}$ representative for the Eiffel Tower pixel. We then applied the empirical relationship between surface and column $\mathrm{NO}_{2}$ values established from 2 years of coincident column and in situ measurements over Paris by Dieudonné et al. (2013). This empirical relationship relates $\mathrm{NO}_{2}$ surface concentrations to the $\mathrm{NO}_{2}$ column $\left(\mathrm{N}_{\mathrm{AP}}\right)$ via the boundary layer height:

$$
N_{\mathrm{AP}}=K\left(0.244 h\left(C_{g}-1.38\right)+0.184\left(C_{g}-2.83\right)\right)
$$

with $\mathrm{K}$ a constant factor that converts $1 \mu \mathrm{g} / \mathrm{m}^{3}$ in a $1 \mathrm{~km}$ deep boundary layer into a column of $1.31 \cdot 10^{15}$ molec $\mathrm{cm}^{-2}, \mathrm{C}_{g}$ the surface $\mathrm{NO}_{2}$ concentration (in $\mu \mathrm{g} / \mathrm{m}^{3}$ ), and $\mathrm{h}$ the boundary layer height in $\mathrm{km}$ (from ECMWF). The scaling factor in Eq. S5.1 have been determined by fitting the tropospheric $\mathrm{NO}_{2}$ columns against $\mathrm{NO}_{2}$ surface concentration for different boundary layer height classes, and show that the $\mathrm{NO}_{2}$ columns scale progressively with increasing boundary layer height (Dieudonné et al., 2013).

By applying the above procedure, we obtained 28 'AirParif' $\mathrm{NO}_{2}$ columns measured within 30 minutes of the TROPOMI observations over the Eiffel Tower. On one day, 24 April 2018, there were no $\mathrm{NO}_{2}$ measurements available from AirParif. Figure S5.1 shows very good agreement between the AirParif and TROPOMI $\mathrm{NO}_{2}$ columns $\left(\mathrm{R}^{2}=0.88\right)$. On average TROPOMI $\mathrm{NO}_{2}$ columns are lower than those from AirParif by 10-15\%. To characterize the differences, we did a reduced major axis regression. This suggests that TROPOMI has a small, systematic offset of $+0.8 \cdot 10^{15}$ molec. $\mathrm{cm}^{-2}$, and a multiplicative low-bias component of $25 \%$. The multiplicative component of the low bias suggests that the increases in $\mathrm{NO}_{2}$ columns over Paris are underestimated by the same amount (the offset is discarded in the line density analysis as explained in the Methods). This relative underestimate of $25 \%$ is corrected for in the inversion method.

\section{S5.2 Line densities over Paris}

For each mostly clear-sky day, we calculated maps of $\mathrm{NO}_{2}$ tropospheric columns from S5PTROPOMI observations. We then rotated the maps such that they align with the prevailing ECMWF boundary layer wind direction over Paris. Line densities $\mathrm{L}(\mathrm{x})$ (as function of distance $\mathrm{x}$ from the point source in wind direction) are determined by spatial integration over a $60 \mathrm{~km}$ 

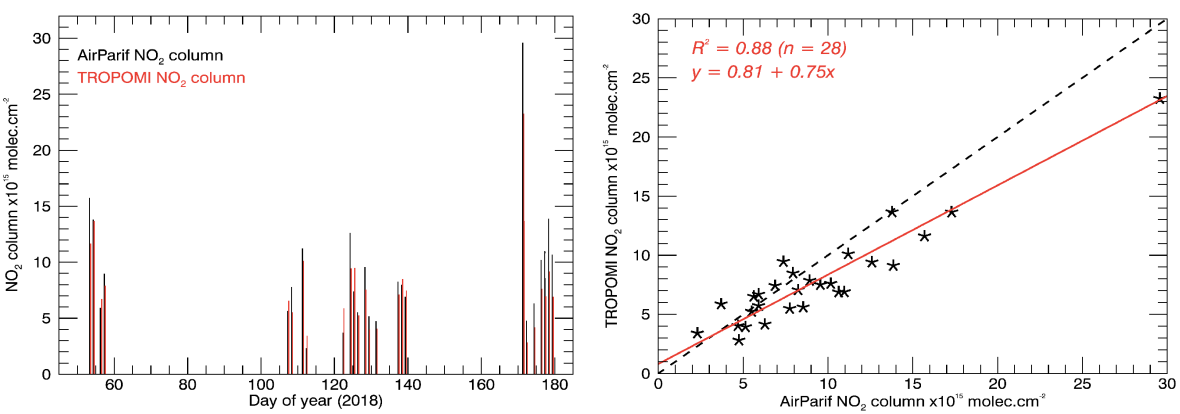

Figure S5.1. Comparison of tropospheric $\mathrm{NO}_{2}$ columns from AirParif (calculated with Eq. S5.1) and TROPOMI over the Eiffel Tower. The left panel shows a time series of the clear-sky columns between February and July 2018. The right panel shows a scatter plot and the results of a reduced major axis regression analysis of TROPOMI vs. AirParif. The average distance of the TROPOMI pixel centre to the Eiffel Tower was $2.6 \mathrm{~km}$.

interval in across-wind direction. The $60 \mathrm{~km}$ interval is motivated by the spatial contours of Paris and the horizontal distribution of bottom-up emissions that both suggest that the entire Paris metropolitan area is enveloped in all directions within a radius of $30 \mathrm{~km}$ (Figure S5.2).

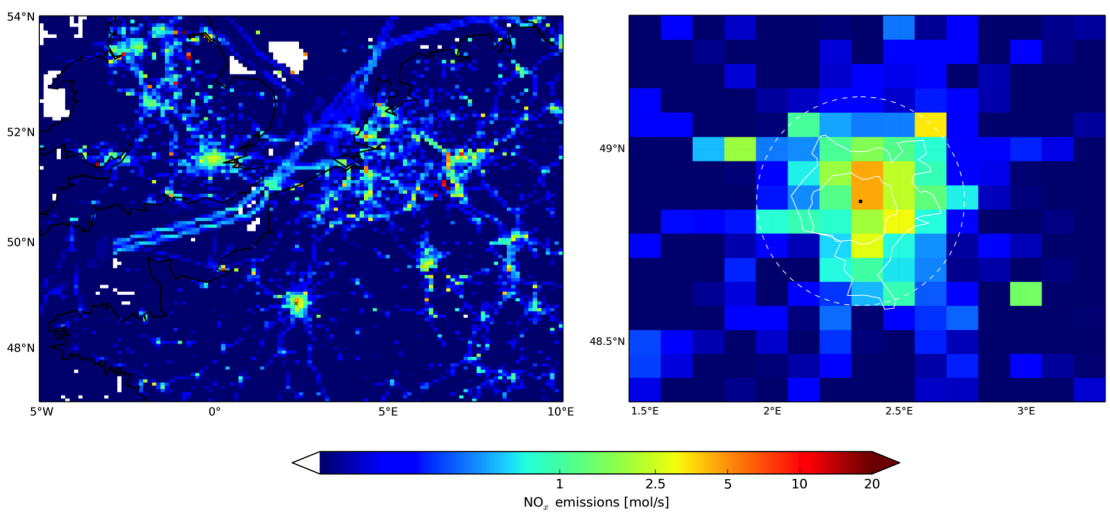

Figure S5.2. Upper panels: $\mathrm{NO}_{x}$ emission strength $\left(\mathrm{mol} \mathrm{s}^{-1}\right)$ over the Paris Metropolitan area from the TNOMACC- III emission inventory for 2011 ; original resolution $1 / 8^{\circ} \times 1 / 16^{\circ}$ (lon $\times$ lat). In the upper right panel, the location of the A86 Super-périphérique Parisien highway and geographical contours of the entire metropolitan area (including Sarcelles, Taverny, Montesson, Versailles, Palaiseau, Bretigny, Villiers sur-Marne, Torcy, Mitry- Mory, and Paris Charles-de-Gaulle airport) are indicated as white solid lines. The $30 \mathrm{~km}$ radius envelope is indicated as a dashed white line.

We focus on the pollution build-up of $\mathrm{NO}_{2}$ in a column of air that is advected over the city. Our line densities thus start $30 \mathrm{~km}$ upwind of the Paris city centre, and end $30 \mathrm{~km}$ downwind. This ensures that line densities capture the full extent of the accumulation of $\mathrm{NO}_{2}$ over the metropolitan area. Figure S5.3 illustrates our method to calculate the line densities, for Friday 4 May 2018. 


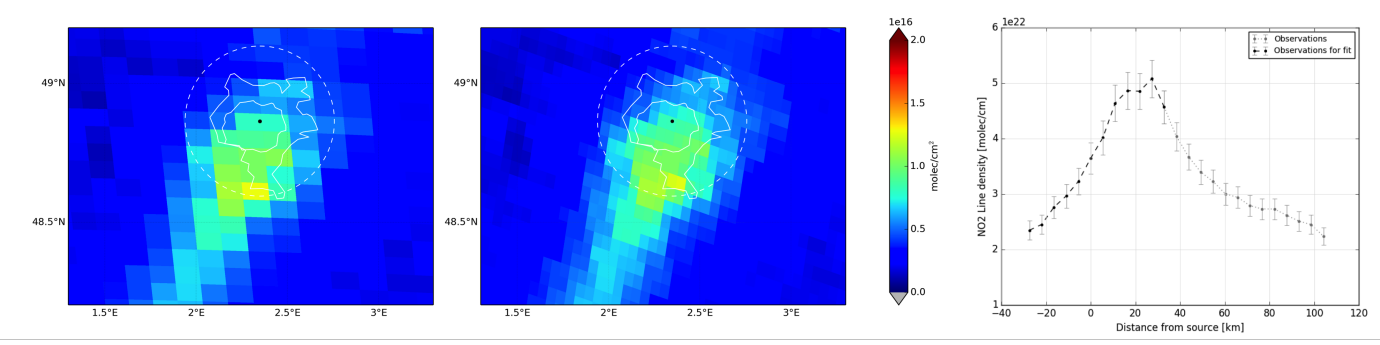

Figure S5.3. Left and middle panel: tropospheric $\mathrm{NO}_{2}$ vertical column on 4th May 2018 from S5P- TROPOMI, S5P- TROPOMI rotated towards North-North-east wind direction. Right panel: corresponding $\mathrm{NO}_{2}$ line density from $0 \mathrm{~km}$ (upwind) to $60 \mathrm{~km}$ (downwind) over Paris (black dashed line) with further downwind line density decay up to $140 \mathrm{~km}$ in light grey.

\section{S5.3 Temporal variation in $\mathrm{NO}_{x}$ emissions}

To account for temporal variations and allow a fair comparison between the inferred S5PTROPOMI $\mathrm{NO}_{x}$ emissions (valid for approximately 12:00 hrs local time) and the $\mathrm{NO}_{x}$ emissions predicted by the TNO-MACC III inventory, we used the emission scaling factors over Paris provided in the TNO-MACC III inventory (Kuenen et al., 2014). Figure S5.4 illustrates the scaling factors that relate the 24-hour mean emissions in the inventory to the emission strength at 12:00 hrs for all the days of the week, and for different months. According to the inventory, weekend reductions are $\pm 30 \%$ in winter, and $\pm 20 \%$ in summer, and emissions are some $10 \%$ lower in the summer months than in winter as a consequence of a smaller contribution from residential heating in the warm season.

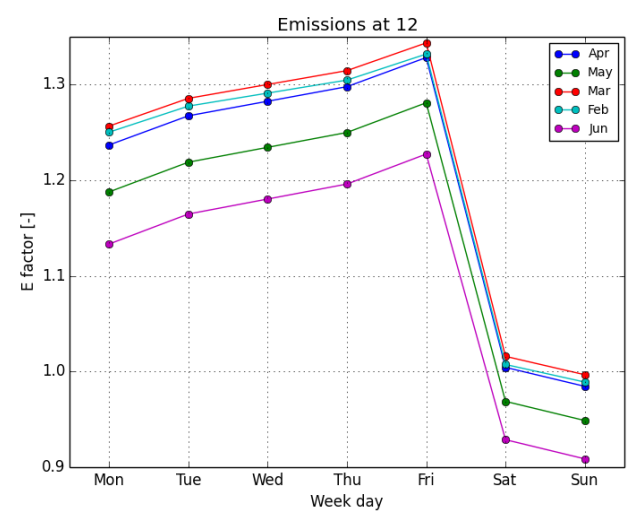

Figure S5.4. Emission scaling factors for 12:00 hrs local time over Paris, France, as provided by the TNO-MACC III inventory. A value of 1.0 corresponds to the 24 -hr mean (for the year 2011) $\mathrm{NO}_{x}$ emissions provided in the inventory.

\section{S5.4 Modeled boundary layer mean $\mathrm{OH}$ concentrations}

Because it is difficult for coarse-resolution chemistry transport models to reproduce $\mathrm{OH}$ concentrations ((Ren et al., 2003), (Valin et al., 2013)), especially over strong source regions such 
as Paris, we allow for a range of $\pm 50 \%$ on the boundary layer mean $\mathrm{OH}$ concentrations around the values predicted by CAMS over the city. This range was motivated by a comparison of $\mathrm{OH}$ simulations by the 3-D CTM CAMS and 0-D CLASS model (Vilà-Guerau de Arellano et al., 2005) showing that $\mathrm{OH}$ levels from the latter are some $50 \%$ lower (Figure S5.5) on most days in June. The CLASS box model was forced with (1) initial conditions; (2) emissions; and (3) a chemical scheme, to reproduce the $\mathrm{O}_{3}$ and $\mathrm{NO}_{x}$ values observed at the $3 \mathrm{rd}$ floor of the Eiffel Tower.

Wednesday 20 June 2018 was a day with extremely low wind speed and a boundary layer reaching an altitude of only $950 \mathrm{~m}$ over Paris. This is associated with a high pressure system and large-scale subsidence, capping the boundary layer. Indeed, the $\mathrm{NO}_{2}$ column at the city centre exceeded $2.5 \cdot 10^{16}$ molec. $\mathrm{cm}^{-2}$ on this day, and $\mathrm{NO}_{2}$ concentrations measured at the top of the Eiffel Tower (3rd floor, $300 \mathrm{~m}$ altitude) just before the TROPOMI overpass were very high (40 $\mu \mathrm{g} / \mathrm{m}^{3}$ compared to $5-15 \mu \mathrm{g} / \mathrm{m}^{3}$ on other June days (Airparif, 2018)), and the boundary layer was not as deep as on other June days. We evaluated this particular situation with the CLASS mixed-layer model of atmospheric chemistry, constrained by measured concentrations of O3, $\mathrm{NO}, \mathrm{NO}_{2}$ and boundary layer height. The CLASS model simulations suggest that daytime $\mathrm{OH}$ was indeed lower by a factor 3-5 on 20 June than in the period between 21 and 28 June, when wind speeds and boundary layer heights were much higher. Figure S5.5 shows that the CAMS $\mathrm{OH}$ simulations correspond to those from CLASS within a factor 2 between 21 and 28 June, but that CAMS does not capture the reduced $\mathrm{OH}$ levels simulated by CLASS for the stagnant situation on 20 June. This can be attributed to fast dispersion of $\mathrm{NO}_{x}$ in that model over the $40 \mathrm{~km} \times 40 \mathrm{~km}$ grid boxes, promoting the formation of $\mathrm{O}_{3}$ and $\mathrm{OH}$, compared to the high- $\mathrm{NO}_{x}$ regime indicated by observations. In contrast, CLASS, constrained by observed $\mathrm{NO}, \mathrm{NO}_{2}$, and $\mathrm{O}_{3}$ concentrations from the Eiffel Tower and boundary layer height from ECMWF, suggests that $\mathrm{OH}$ levels are actually suppressed by the high levels of pollutants $\left(\mathrm{NO}_{x}\right.$, VOCs $)$ that accumulate over Paris on this day without any ventilation and a relatively shallow boundary layer.

We therefore reduced the boundary layer mean OH from CAMS $\left(15 \cdot 10^{6}\right.$ molec. $\left.\mathrm{cm}^{-3}\right)$ by a factor of 4 to obtain a more plausible first guess of the $\mathrm{NO}_{x}$ loss rate constant on this day. The inferred emissions from two successive orbits are on the same order of magnitude as on other June days, but the inferred $\mathrm{NO}_{x}$ lifetime is somewhat longer. Both estimates come with considerable uncertainties because of the low wind speed (Table S5.1).

\section{S5.5 Uncertainties}

The S5P-TROPOMI $\mathrm{NO}_{x}$ emissions derived from S5P-TROPOMI are inherently uncertain. Here we discuss and quantify the main contributions. Our estimated emissions are directly affected by the accuracy of the S5P-TROPOMI columns, which is driven by structural uncertainty in the tropospheric air mass factor calculation (Boersma et al. (2018), Lorente et al. (2017) and amounts to $\pm 30 \%$.

The ratio between $\mathrm{NO}_{2}$ and $\mathrm{NO}_{x}$ concentrations from CAMS over Paris has an uncertainty of 


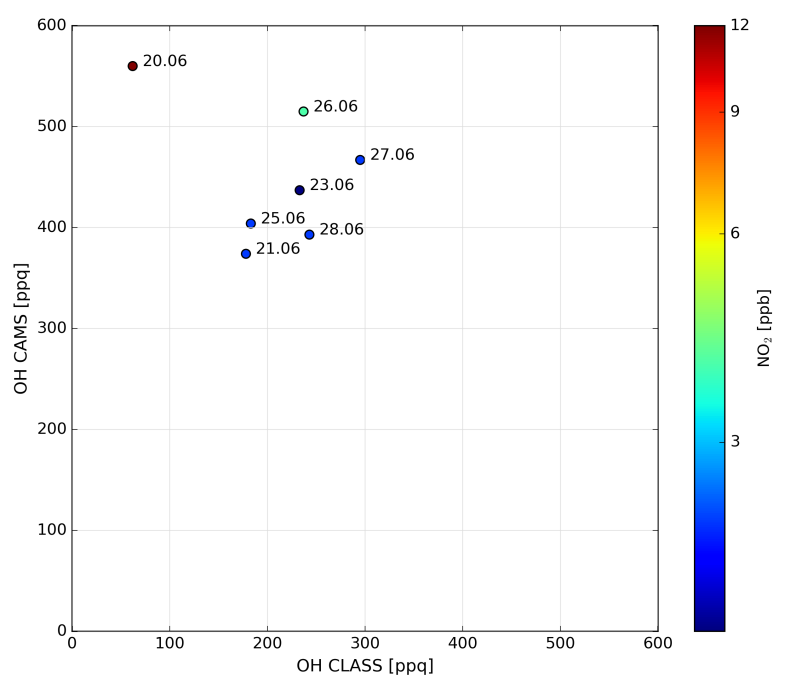

Figure S5.5. Scatter-plot of OH simulated by the $40 \times 40 \mathrm{~km}^{2}$ CAMS CTM ensemble mean and boundary layer CLASS model at the TROPOMI overpass time (12:00-14:00 hrs; constrained by measurements from AirParif, Eiffel Tower station, 3rd etage). The corresponding $\mathrm{NO}_{2}$ concentrations observed at the Eiffel Tower are colour-coded.

$\pm 20 \%$, based on a comparison of CAMS simulations and observations at the Eiffel Tower (Table 1). If we replace CAMS $\mathrm{NO}_{x}$-to- $\mathrm{NO}_{2}$ ratio's (geometric mean: 1.36) by those from the Eiffel Tower (geometric mean: 1.43), our emission estimates are reduced by less than $3 \%$ on average. We adopt an error of $\pm 20 \%$ in the ECMWF boundary layer mean wind speed, consistent with (Beirle et al., 2011), and (Petetin et al., 2015). With this error, we found that emissions changed by $+20 \%$, when modifying the wind speed by $+20 \%$.

Our results are only weakly sensitive to assumptions on the $\mathrm{NO}_{x}$ lifetime. Firstly, our method allows for an uncertainty in the boundary layer mean $\mathrm{OH}$ concentrations from CAMS of $50 \%$, reflecting the difficulty that models have in capturing $\mathrm{OH}$ over a large city. Because the inferred emissions have some dependency on the selected range of possible $\mathrm{OH}$ concentrations, we did a test where we used CLASS OH concentrations (with generally lower values, e.g. Figure S5.5 as our starting point instead of the CAMS values. Results change by less than $15 \%$, thus our method proves to be quite robust for such changes. If CAMS systematically overestimates the boundary layer $\mathrm{OH}$, then emissions will be overestimated.

The a priori emission pattern is important to minimize the differences between the observed and modelled line densities, but its exact shape is of little influence to the overall emissions. We did an experiment where we used one fixed a priori emission pattern as predicted by the TNOMACC-III inventory, and compared the resulting emissions to the results obtained by optimizing the pattern as described in the manuscript. The emissions are similar to within $10 \%$.

Other uncertainties include wind direction and wind speed changing with height. Our method assumes that the $\mathrm{NO}_{2}$ pollution over Paris is advected in a well-defined direction and that the 
boundary layer average wind speed captures the advection well. In reality there will be differences in wind direction and wind speed with altitude. The effects of wind direction uncertainties on $\mathrm{NO}_{x}$ emissions are likely limited, because the $60 \mathrm{~km}$ intervals for calculating line densities are large enough to absorb small errors in wind direction. Wind speed variations with height do not lead to large uncertainties in the estimated emissions. As described in the Methods, the $\mathrm{NO}_{2}{ }^{-}$ weighted mean boundary layer wind speed did not differ significantly from the mean wind speed between the surface and the boundary layer depth, so that the mean boundary layer wind speed can be considered to be representative for the bulk of the $\mathrm{NO}_{2}$ in the boundary layer.

Table S5.1. Evaluation of uncertainties in the individual components and how these affect the $\mathrm{NO}_{x}$ emission estimates from S5P-TROPOMI.

\begin{tabular}{lll}
\hline & Uncertainty & Effect on $\mathrm{NO}_{x}$ emissions \\
\hline $\mathrm{S} 5 \mathrm{P}-\mathrm{TROPOMI} \mathrm{NO}_{2}$ column & $30 \%$ & $30 \%$ \\
\hline $\mathrm{NO}_{2}: \mathrm{NO}_{x}$ ratio & $20 \%$ & $3 \%$ \\
\hline Wind speed & $20 \%$ & $20 \%$ \\
\hline A priori $\mathrm{NO}_{x}$ loss rate & $50 \%$ & $15 \%$ \\
\hline A prioir emission pattern & $20 \%$ & $10 \%$ \\
\hline \hline $\begin{array}{l}\text { Total uncertainties assuming } \\
\text { uncorrelated error contributions }\end{array}$ & $50 \%$ \\
\hline
\end{tabular}

Systematic wind variations, for instance a relatively fast change in the prevailing wind direction, or recirculation, may affect the spatial build-up patterns over the city, and thus the fit results. Often this effect is small, and the observed patterns are dominated by the wind conditions close to the time of the satellite measurement. Days with rapid changes in wind direction or wind speed such as 19 April 2018 have been excluded from further analysis (see Methods).

The total uncertainty in the $\mathrm{NO}_{x}$ emissions is mostly driven by the uncertainties in the S5P-TROPOMI $\mathrm{NO}_{2}$ columns, and contains non-negligible contributions from uncertainty in wind speed and a priori assumptions on $\mathrm{NO}_{x}$ loss rate and emission patterns. We add these contributions in quadrature and estimate an overall emission uncertainty of 36-65\%. These estimates are higher for days with low wind speeds, when wind speed and $\mathrm{OH}$ are particularly uncertain, such as on 20 June 2018 when we estimate uncertainties of $\pm 100 \%$. The fit results are robust with respect to changes in the selection of the across-city integration interval, as long as this interval is chosen wide enough $(60 \mathrm{~km}$, all of Paris is captured within a $30 \mathrm{~km}$ radius). If this interval is chosen more narrowly, then the line density will not cover the full across-wind extent of Paris, so that the emissions would be representative for a smaller part of Paris.

\section{S5.6 Spatial distribution of the estimated $\mathrm{NO}_{x}$ emissions}

Figure S5.6 compares the (1-D) emission distribution from TROPOMI and TNO-MACC III over Paris for all week and weekend days. The patterns agree reasonably well, and indicate that the bulk of the Parisian $\mathrm{NO}_{x}$ is indeed emitted within a radius of $15 \mathrm{~km}$ of the city centre, with weaker contributions from the suburbs. 

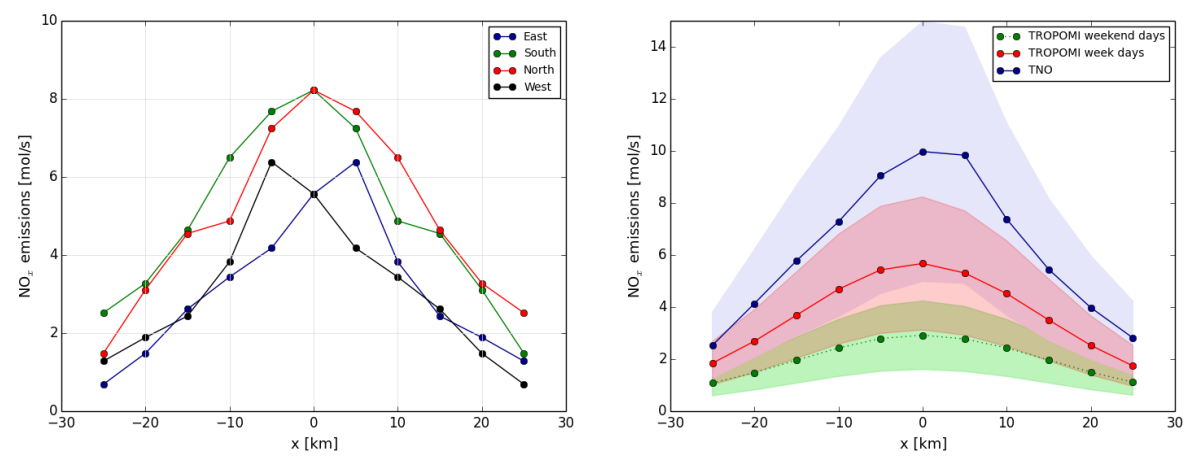

Figure S5.6 Left panel: emission 'line density', obtained by accumulating TNO-MACCIII $\mathrm{NO}_{x}$ emissions across Paris over a $60 \mathrm{~km}$ perpendicular to the wind in steps of $5 \mathrm{~km}$. Right panel: average $\mathrm{NO}_{x}$ emission line density (mol/s) from TROPOMI weekdays (red) and weekend days (green) and TNO-MACC-III (dark blue) over Paris. The TNO-MACC-III emission line densities were calculated by sampling along the main wind direction, and hold for the samples days in the year 2011. The TROPOMI patterns have not yet been corrected for the low bias discussed at the beginning of this section.

\section{S5.7 S5P-TROPOMI $\mathrm{NO}_{2}$ maps, line densities, and model fits for all days}

Figure S5.7 below shows the S5P-TROPOMI tropospheric $\mathrm{NO}_{2}$ columns (left columns) and the observed and fitted $\mathrm{NO}_{2}$ line densities (right columns) for all days that were mostly cloud-free and had valid data over Paris in the period February-June 2018. 

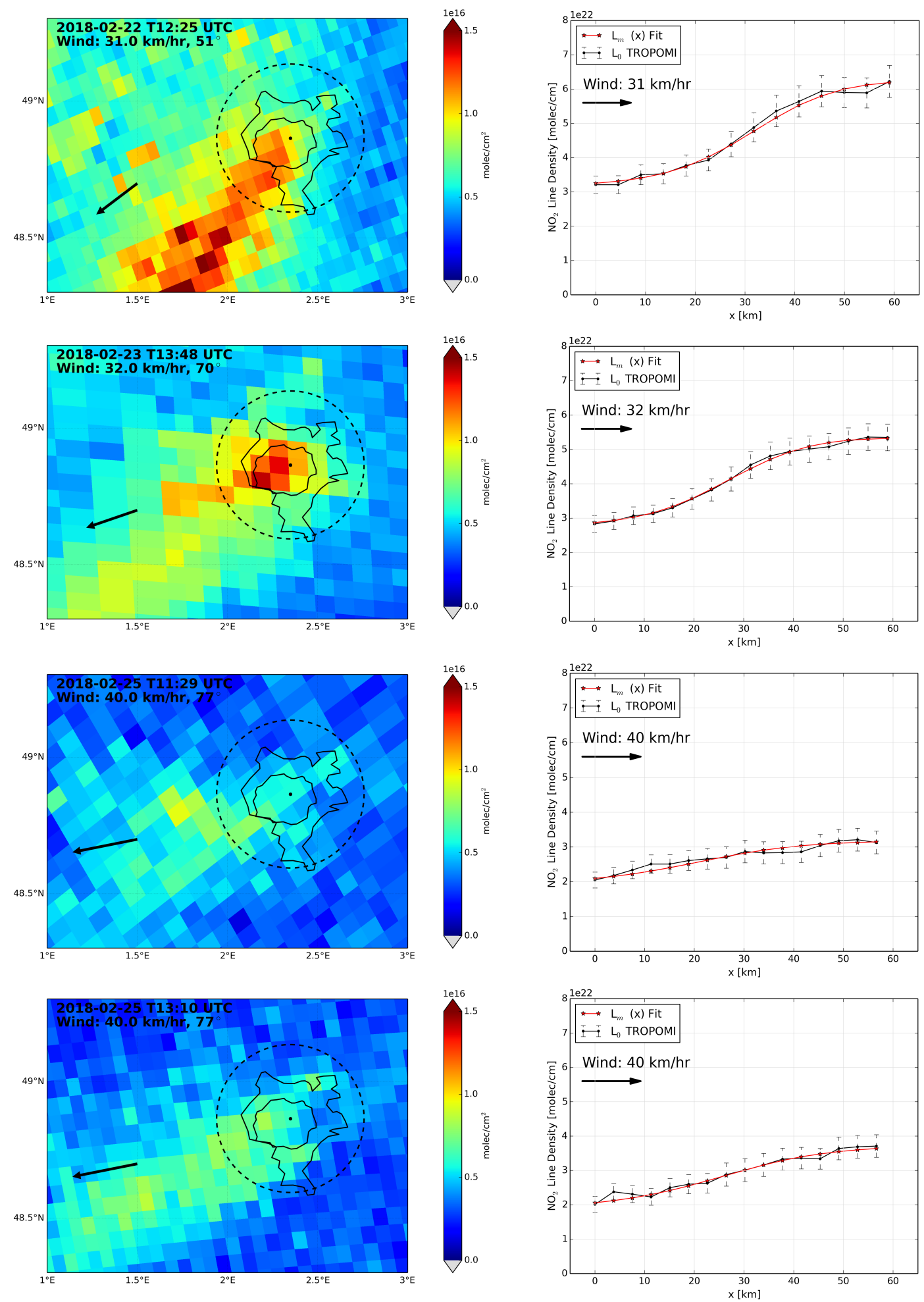

Figure S5.7. Tropospheric $\mathrm{NO}_{2}$ columns for all clear-sky days as observed by TROPOMI in the period February-June 2018 (left panels) and the corresponding observed and modelled $\mathrm{NO}_{2}$ line densities. The black arrow in the left panels indicates the wind direction, and the length of the arrow is proportional to the wind speed. 

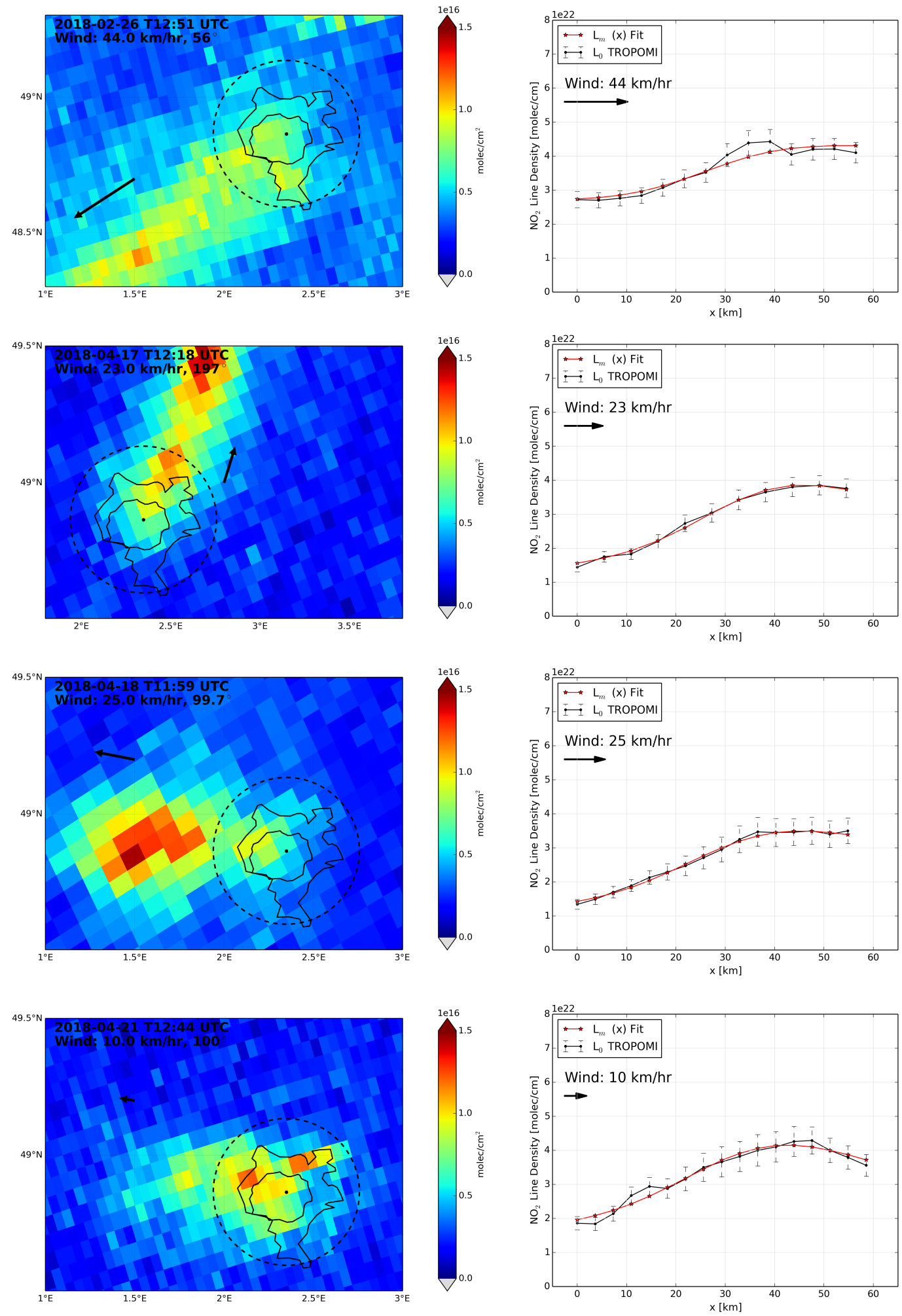

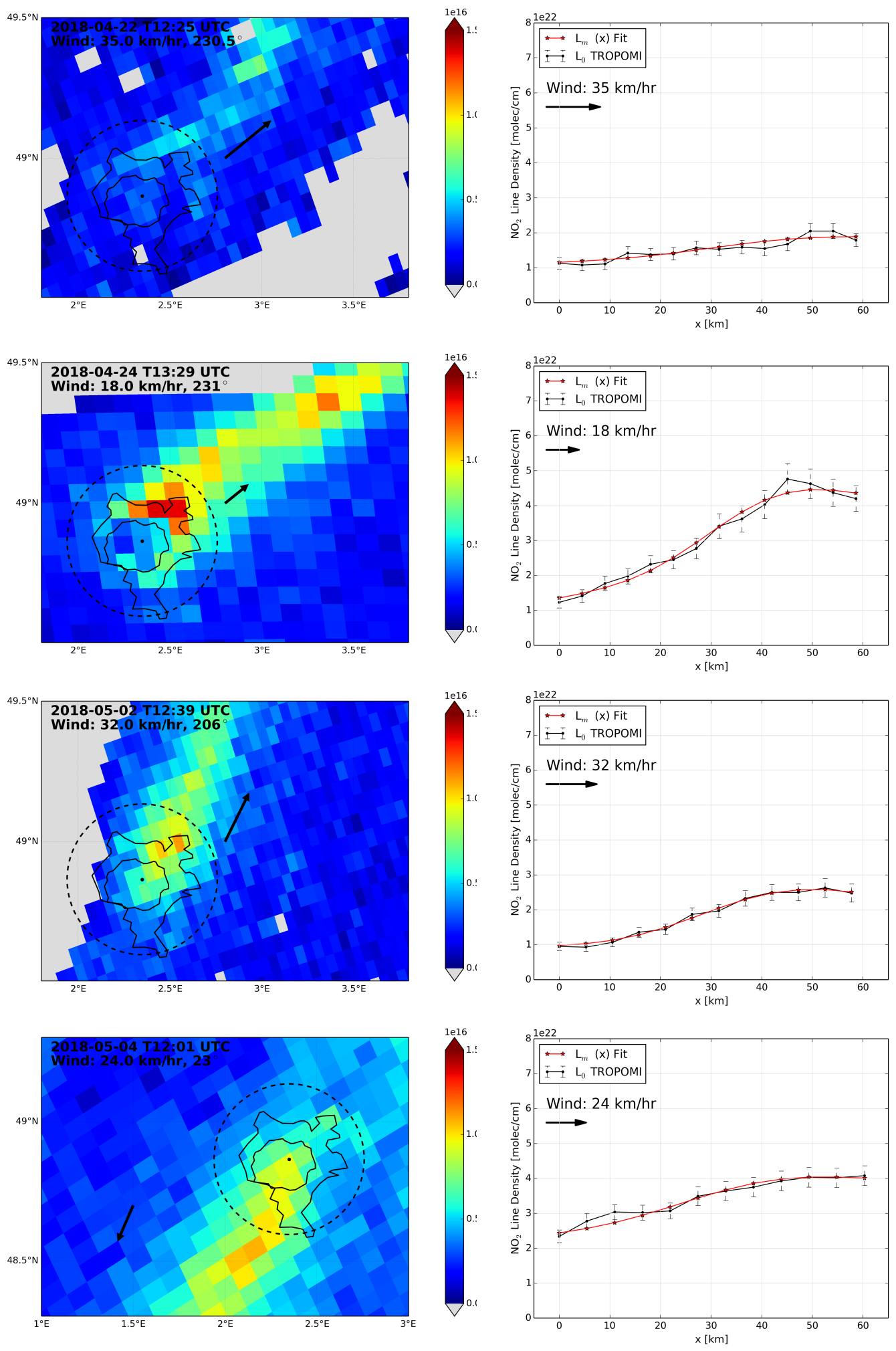

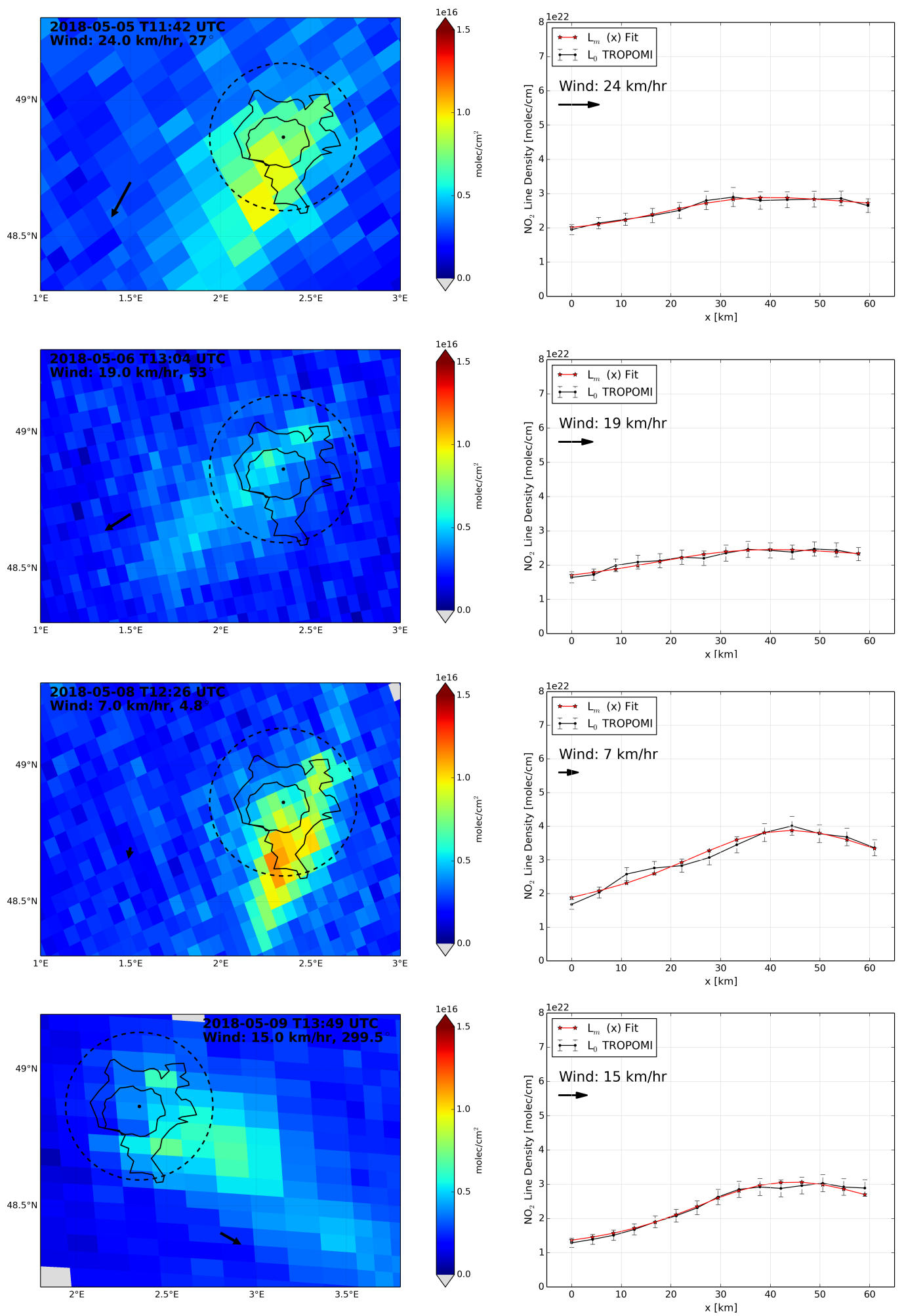

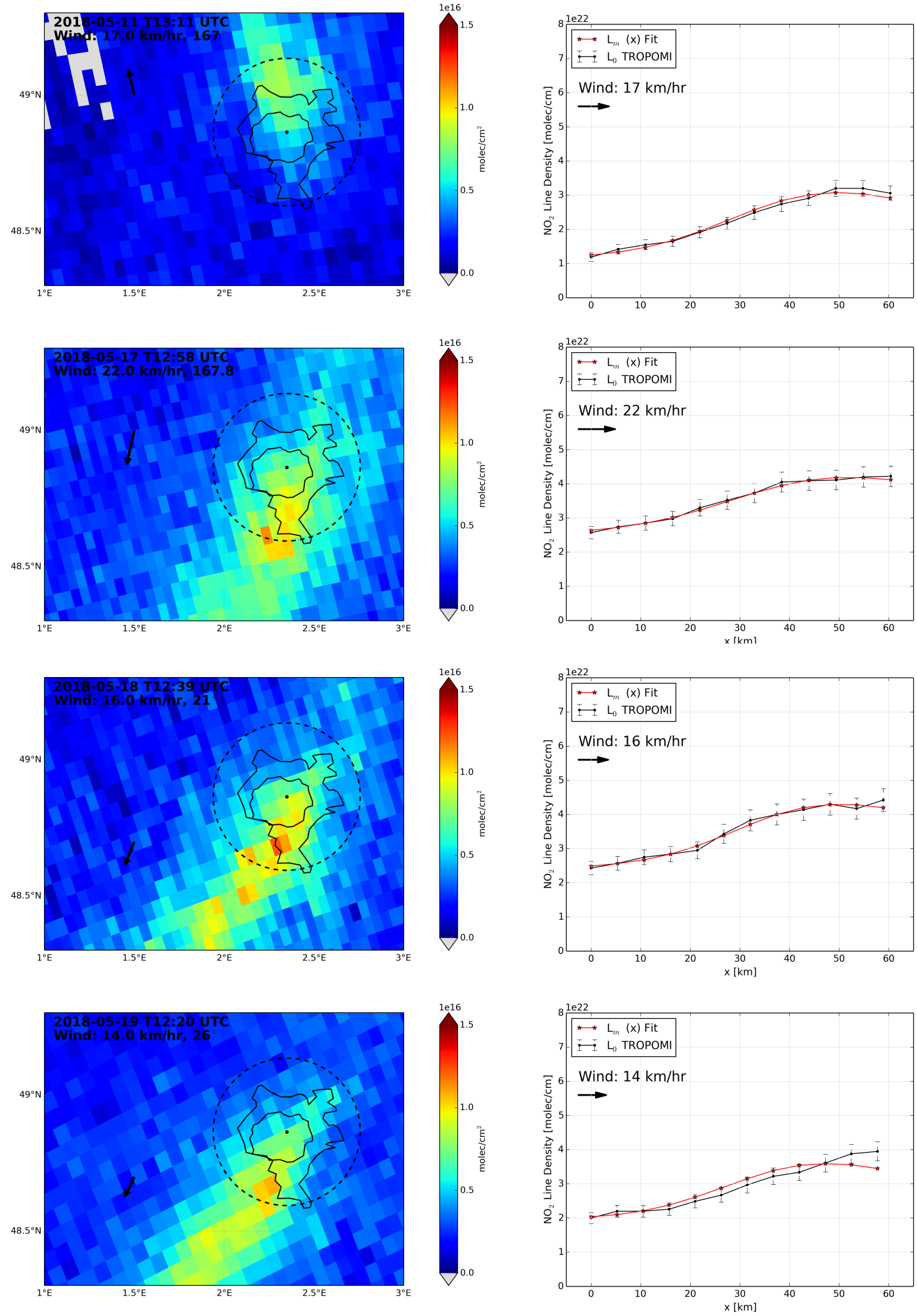

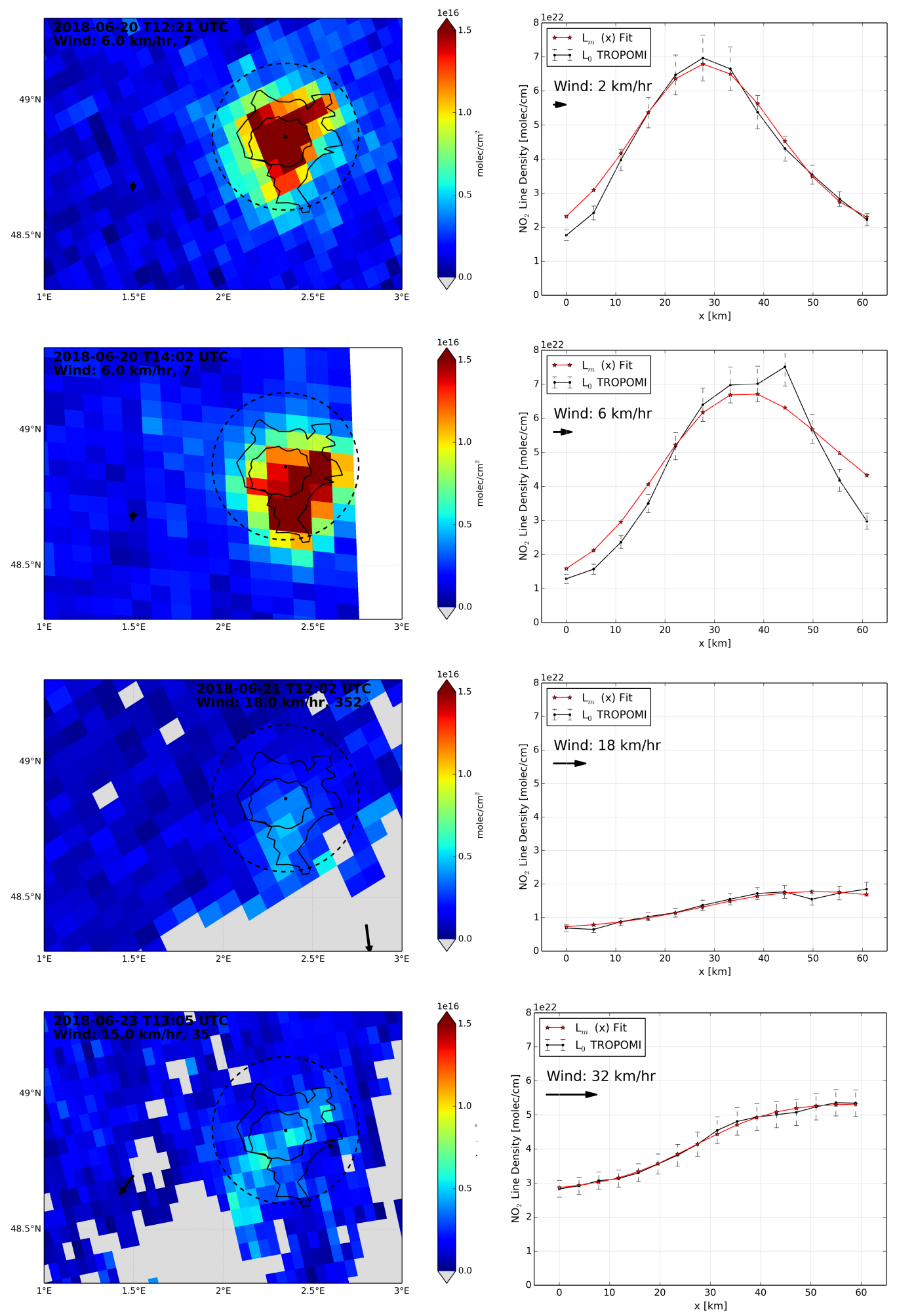

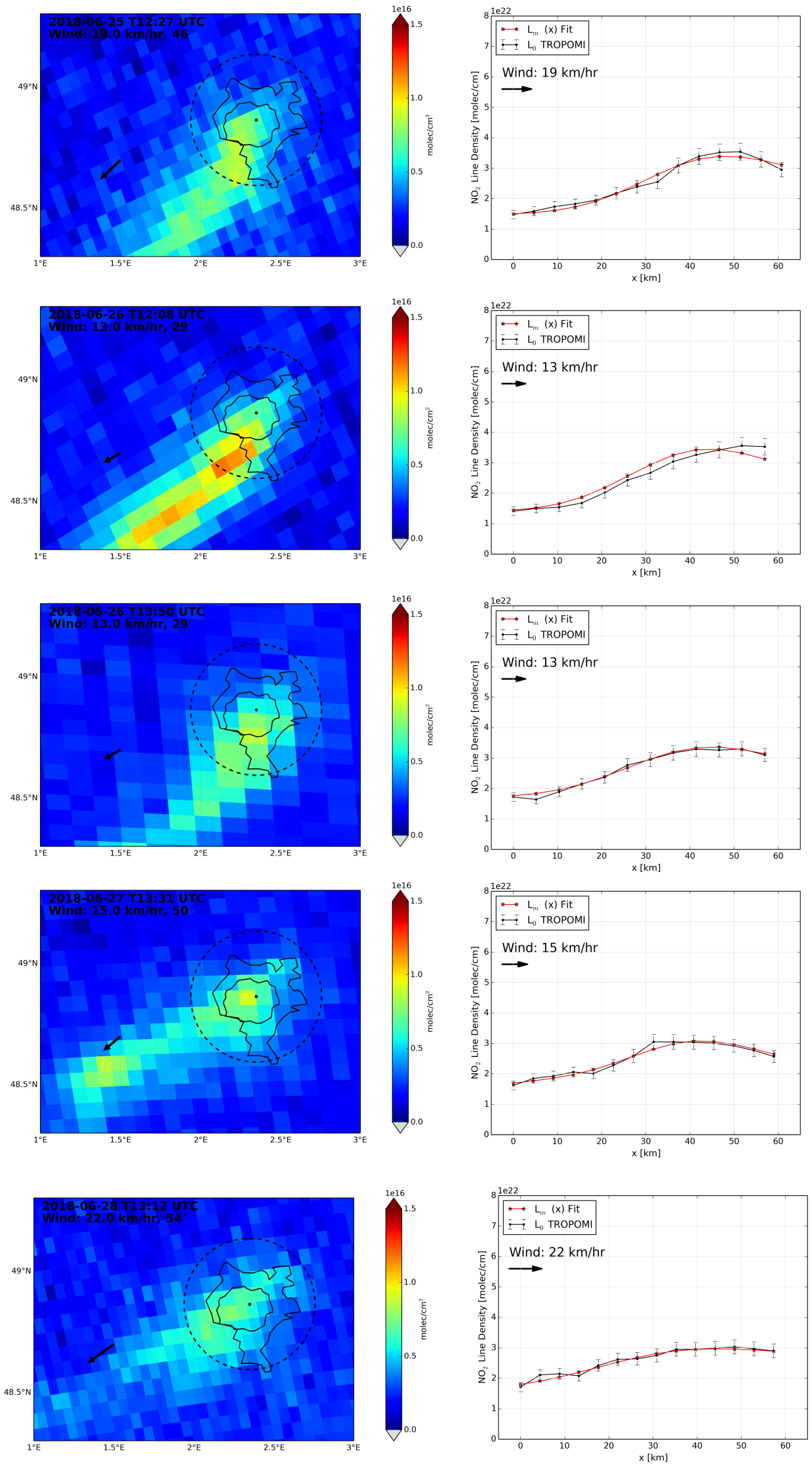



\section{References}

Acarreta, J. R., De Haan, J. F., and Stammes, P.: Cloud pressure retrieval using the O2-O2 absorption band at $477 \mathrm{~nm}$, J. Geophys. Res.: Atmospheres, 109, n/a-n/a, doi: 10.1029/2003JD003915, d05204, 2004.

Airparif: Air quality in the Paris region - Summary 2017, Tech. rep., Air quality observatory in the Paris region (AIRPARIF), complete report in French: http://www.airparif.asso.fr/_pdf/publications/bilan2016.pdf, 2017.

Airparif: Airparf station data, Online, http://www.airparif.asso.fr/, 2018.

Anderson, G. P., Clough, S. A., Kneizys, F. X., Chetwynd, J. H., and Shettle, E. P.: AFGL Atmospheric Constituent Profiles (0-120km), Environ. Res. Papers, 1986.

Barkley, M. P., Palmer, P. I., Ganzeveld, L., Arneth, A., Hagberg, D., Karl, T., Guenther, A., Paulot, F., Wennberg, P. O., Mao, J., Kurosu, T. P., Chance, K., Müller, J.-F., De Smedt, I., Van Roozendael, M., Chen, D., Wang, Y., and Yantosca, R. M.: Can a "state of the art" chemistry transport model simulate Amazonian tropospheric chemistry?, J. Geophys. Res., 116, doi: 10.1029/2011JD015893, d16302, 2011.

Barkley, M. P., Kurosu, T. P., Chance, K., De Smedt, I., Van Roozendael, M., Arneth, A., Hagberg, D., and Guenther, A.: Assessing sources of uncertainty in formaldehyde air mass factors over tropical South America: Implications for top-down isoprene emission estimates, J. Geophys. Res., 117, doi: 10.1029/2011JD016827, 2012.

Barkley, M. P., De Smedt, I., Van Roozendael, M., Kurosu, T. P., Chance, K., Arneth, A., Hagberg, D., Guenther, A., Paulot, F., Marais, E., and Mao, J.: Top-down isoprene emissions over tropical South America inferred from SCIAMACHY and OMI formaldehyde columns, J. Geophys. Res., 118, 6849-6868, doi: 10.1002/jgrd.50552, 2013.

Bauwens, M., Stavrakou, T., Müller, J.-F., De Smedt, I., Van Roozendael, M., van der Werf, G. R., Wiedinmyer, C., Kaiser, J. W., Sindelarova, K., and Guenther, A.: Nine years of global hydrocarbon emissions based on source inversion of OMI formaldehyde observations, Atmospheric Chemistry and Physics, 16, 10 133-10 158, doi: 10.5194/acp-16-10133-2016, 2016.

Behrens, L. K., Hilboll, A., Richter, A., Peters, E., Eskes, H., and Burrows, J. P.: GOME-2A retrievals of tropospheric $\mathrm{NO}_{2}$ in different spectral ranges - influence of penetration depth, Atmospheric Measurement Techniques, 11, 2769-2795, doi: 10.5194/amt-11-2769-2018, 2018.

Beirle, S.: Background correction Best Practices and Recommendations, Sect. 2.4 of QA4ECV Deliverable 4.2 version 1.0, URL http://www.qa4ecv.eu/sites/default/files/D4.2.pdf, last access : June 2018, 2016.

Beirle, S., Huntrieser, H., and Wagner, T.: Direct satellite observation of lightning-produced $\mathrm{NO}_{x}$, Atmos. Chem. Phys., 10, 10 965-10 986, doi: 10.5194/acp-10-10965-2010, 2010. 
Beirle, S., Boersma, K. F., Platt, U., Lawrence, M. G., and Wagner, T.: Megacity Emissions and Lifetimes of Nitrogen Oxides Probed from Space, Science, 333, 1737-1739, doi: 10.1126/science.1207824, 2011.

Beirle, S., Hörmann, C., Jöckel, P., Penning de Vries, M., Pozzer, A., Sihler, H., Valks, P., and Wagner, T.: The STRatospheric Estimation Algorithm from Mainz (STREAM): Estimating stratospheric $\mathrm{NO}_{2}$ from nadir-viewing satellites by weighted convolution, Atmos. Meas. Tech. Discuss., 2016, 1-46, doi: 10.5194/amt-2015-405, 2016.

Berrisford, P., Kållberg, P., Kobayashi, S., Dee, D., Uppala, S., Simmons, A. J., Poli, P., and Sato, H.: Atmospheric conservation properties in ERA-Interim, Quarterly Journal of the Royal Meteorological Society, 137, 1381-1399, doi: 10.1002/qj.864, 2011.

Boersma, K. F., Bucsela, E. J., Brinksma, E. J., and Gleason, J. F.: $\mathrm{NO}_{2}$, in: OMI Algorithm Theoretical Basis Document, Tech. rep., NASA Distrib. Active Archive Cent., 2002.

Boersma, K. F., Eskes, H. J., and Brinksma, E. J.: Error analysis for tropospheric $\mathrm{NO}_{2}$ retrieval from space, J. Geophys. Res., 109, D04 311, doi: 10.1029/2003JD003962, 2004.

Boersma, K. F., Eskes, H. J., Meijer, E. W., and Kelder, H. M.: Estimates of lightning $\mathrm{NO}_{x}$ production from GOME satellite observations, Atmospheric Chemistry and Physics, 5, 2311-2331, doi: 10.5194/acp-5-2311-2005, 2005.

Boersma, K. F., Jacob, D. J., Eskes, H. J., Pinder, R. W., Wang, J., and van der A, R. J.: Intercomparison of SCIAMACHY and OMI tropospheric $\mathrm{NO}_{2}$ columns: Observing the diurnal evolution of chemistry and emissions from space, Journal of Geophysical Research: Atmospheres, 113, doi: 10.1029/2007JD008816, 2008.

Boersma, K. F., Eskes, H. J., Dirksen, R. J., van der A, R. J., Veefkind, J. P., Stammes, P., Huijnen, V., Kleipool, Q. L., Sneep, M., Claas, J., Leitao, J., Richter, A., Zhou, Y., and Brunner, D.: An improved tropospheric $\mathrm{NO}_{2}$ column retrieval algorithm for the Ozone Monitoring Instrument, Atmos. Meas. Tech., 4, 1905-1928, doi: 10.5194/amt-4-1905-2011, 2011.

Boersma, K. F., Vinken, G. C. M., and Tournadre, J.: Ships going slow in reducing their $\mathrm{NO}_{x}$ emissions: changes in 2005-2012 ship exhaust inferred from satellite measurements over Europe, Environ. Res. Lett., 10, 074 007, doi: doi:10.1088/1748-9326/10/7/074007, 2015.

Boersma, K. F., Eskes, H., Ding, J., van der A, R., Miyazaki, K., Visser, A., Ganzeveld, L., Georgoulias, A., Bauwens, M., Trissevgeni Stavrakou, T., Compernolle, S., Verhoelst, T., Hubert, D., van Roozendael, M., Hendrick, F., Lambert, J., Muller, J. F., George, M., Coheur, P., and Clerbaux, C.: Report on the impact of Atmospheric ECV records on model evaluation, data assimilation, emission and trend estimates (QA4ECV Deliverable 6.3 version 1.0), URL http://www.qa4ecv.eu/sites/default/files/D4.2.pdf, last access : Dec 2018, 2016a.

Boersma, K. F., Vinken, G. C. M., and Eskes, H. J.: Representativeness errors in comparing chemistry transport and chemistry climate models with satellite UV-Vis tropospheric column retrievals, Geosci. Mod. Dev., 9, 875-898, doi: 10.5194/gmd-9-875-2016, 2016b.

Boersma, K. F., Eskes, H., Richter, A., De Smedt, I., Lorente, A., Beirle, S., Zara, M., Peters, E., Van Roozendael, M., Wagner, T., Maasakkers, J., van der A, R., Nightingale, J., De Rudder, A., Irie, H., Pinardi, G., Lambert, J. C., and Compernolle, S.: Improving algorithms and uncertainty estimates for satellite $\mathrm{NO}_{2}$ retrievals: Results from the Quality Assurance for Essential Climate Variables (QA4ECV) project, Atmos. Meas. Tech. Discuss, 2, 10-30, 2018.

Bojinski, S., Verstraete, M., Peterson, T. C., Richter, C., Simmons, A., and Zemp, M.: The Concept of Essential Climate Variables in Support of Climate Research, Applications, and Policy, Bull. Amer. Meteor. Soc., 95, 1431-1443, doi: 10.1175/BAMS-D-13-00047.1, 2014. 
Borsdorff, T., Aan de Brugh, J., Hu, H., Aben, I., Hasekamp, O., and Landgraf, J.: Measuring Carbon Monoxide With TROPOMI: First Results and a Comparison With ECMWF-IFS Analysis Data, Geophysical Research Letters, 45, 2826-2832, doi: 10.1002/2018GL077045, 2018.

Bovensmann, H., Burrows, J. P., Buchwitz, M., Frerick, J., Noël, S., Rozanov, V. V., Chance, K. V., and Goede, A. P. H.: SCIAMACHY: Mission Objectives and Measurement Modes, Journal of the Atmospheric Sciences, 56, 127-150, 1999.

Bucsela, E. J., Perring, A. E., Cohen, R. C., Boersma, K. F., Celarier, E. A., Gleason, J. F., Wenig, M. O., Bertram, T. H., Wooldridge, P. J., Dirksen, R., and Veefkind, J. P.: Comparison of tropospheric NO2 from in situ aircraft measurements with near-real-time and standard product data from OMI, Journal of Geophysical Research: Atmospheres, 113, doi: 10.1029/2007JD008838, 2008.

Bucsela, E. J., Krotkov, N. A., Celarier, E. A., Lamsal, L. N., Swartz, W. H., Bhartia, P. K., Boersma, K. F., Veefkind, J. P., Gleason, J. F., and Pickering, K. E.: A new stratospheric and tropospheric $\mathrm{NO}_{2}$ retrieval algorithm for nadir-viewing satellite instruments: applications to OMI, Atmos. Meas. Tech., 6, 2607-2626, doi: 10.5194/amt-6-2607-2013, 2013.

Burrows, J. P., Weber, M., Buchwitz, M., Rozanov, V., Ladstätter-Weißenmayer, A., Richter, A., DeBeek, R., Hoogen, R., Bramstedt, K., Eichmann, K.-U., Eisinger, M., and Perner, D.: The Global Ozone Monitoring Experiment (GOME): Mission Concept and First Scientific Results, Journal of the Atmospheric Sciences, 56, 151-175, doi: 10.1175/1520-0469(1999)056<0151:TGOMEG>2.0.CO;2, 1999 .

Camacho-de Coca, F., Bréon, F. M., Leroy, M., and Garcia-Haro, F. J.: Airborne measurement of hot spot reflectance signatures, Remote Sensing of Environment, 90, 63 - 75, doi: https://doi.org/10.1016/j.rse.2003.11.019, 2004.

Castellanos, P., Boersma, K. F., and van der Werf, G. R.: Satellite observations indicate substantial spatiotemporal variability in biomass burning $\mathrm{NO}_{x}$ emission factors for South America, Atmospheric Chemistry and Physics, 14, 3929-3943, doi: 10.5194/acp-14-3929-2014, 2014.

Castellanos, P., Boersma, K. F., Torres, O., and de Haan, J. F.: OMI tropospheric $\mathrm{NO}_{2}$ air mass factors over South America: effects of biomass burning aerosols, Atmos. Meas. Tech., 8, 3831-3849, doi: 10.5194/amt-8-3831-2015, 2015.

Celarier, E. A., Brinksma, E. J., Gleason, J. F., Veefkind, J. P., Cede, A., Herman, J. R., Ionov, D., Goutail, F., Pommereau, J.-P., Lambert, J.-C., van Roozendael, M., Pinardi, G., Wittrock, F., Schönhardt, A., Richter, A., Ibrahim, O. W., Wagner, T., Bojkov, B., Mount, G., Spinei, E., Chen, C. M., Pongetti, T. J., Sander, S. P., Bucsela, E. J., Wenig, M. O., Swart, D. P. J., Volten, H., Kroon, M., and Levelt, P. F.: Validation of Ozone Monitoring Instrument nitrogen dioxide columns, J. Geophys. Res.: Atmospheres, 113, n/a-n/a, doi: 10.1029/2007JD008908, d15S15, 2008.

Chimot, J., Vlemmix, T., Veefkind, J. P., de Haan, J. F., and Levelt, P. F.: Impact of aerosols on the OMI tropospheric $\mathrm{NO}_{2}$ retrievals over industrialized regions: how accurate is the aerosol correction of cloudfree scenes via a simple cloud model?, Atmos. Meas. Tech., 9, 359-382, doi: 10.5194/amt-9-359-2016, 2016.

Chimot, J., Veefkind, J. P., de Haan, J. F., Stammes, P., and Levelt, P. F.: Minimizing aerosol effects on the OMI tropospheric $\mathrm{NO}_{2}$ retrieval - An improved use of the $477 \mathrm{~nm} \mathrm{O}_{2}-\mathrm{O}_{2}$ band and an estimation of the aerosol correction uncertainty, Atmospheric Measurement Techniques Discussions, 2018, 1-41, doi: 10.5194/amt-2018-247, URL https://www. atmos-meas-tech-discuss .net/amt-2018-247/, 2018.

Cohen, R. C. and Murphy, J. G.: Photochemistry of $\mathrm{NO}_{2}$ in Earth's Stratosphere : Constraints from Observations, Chemical Reviews, 103, 4985-4998, doi: 10.1021/cr020647x, 2003. 
Compernolle, S., Verhoelst, T., Pinardi, G., Granville, J., Lambert, J.-C., and Eichmann, K.-U.: S5P MPC VDAF Validation Web Article: Nitrogen Dioxide, Tech. Rep. S5P-MPC-VDAF-WVAL2_NO2_20180904, 2018.

Cox, C. and Munk, W.: Measurement of the Roughness of the Sea Surface from Photographs of the Sun's Glitter, J. Opt. Soc. Am., 44, 838-850, doi: 10.1364/JOSA.44.000838, URL http://www. osapublishing. org/abstract.cfm?URI=josa-44-11-838, 1954.

Crippa, M., Guizzardi, D., Muntean, M., Schaaf, E., Dentener, F., van Aardenne, J. A., Monni, S., Doering, U., Olivier, J. G. J., Pagliari, V., and Janssens-Maenhout, G.: Gridded emissions of air pollutants for the period 1970-2012 within EDGAR v4.3.2, Earth System Science Data, 10, 19872013, doi: 10.5194/essd-10-1987-2018, 2018.

Danckaert, T., Fayt, C., Van Roozendael, M., De Smedt, I., Letocart, V., Merlaud, A., , and Pinardi, G.: QDOAS Software user manual, Belgian Institute for Space Aeronomy (BIRA-IASB), version 3.2, URL http://uv-vis . aeronomie.be/software/QDOAS/, 2017.

de Haan, J. F., Bosma, P. B., and Hovenier, J. W.: The adding method for multiple scattering calculations of polarized light, Astron. Astrophys, 183, 371-391, 1987.

De Smedt, I.: Long-Term Global Observations of Tropospheric Formaldehyde Retrieved from Spaceborne Nadir UV Sensors, Ph.D. thesis, Universite Libre de Bruxelles, 2011.

De Smedt, I., Muller, J. F., Stavrakou, T., van der A, R., Eskes, H., and Van Rooezendael, M.: Twelve years of global observation of formaldehyde in the troposphere using GOME and SCIAMACHY sensors, Atmos. Meas. Tech., 8, 4947-4963, doi: 10.5194/acp-8-4947-2008, 2008.

De Smedt, I., Stavrakou, T., Müller, J.-F., van der A, R. J., and Van Roozendael, M.: Trend detection in satellite observations of formaldehyde tropospheric columns, Geophys. Res. Lett., 37, doi: 10.1029/2010GL044245, 2010.

De Smedt, I., Van Roozendael, M., Stavrakou, T., Müller, J.-F., Lerot, C., Theys, N., Valks, P., Hao, N., and van der A, R.: Improved retrieval of global tropospheric formaldehyde columns from GOME2/MetOp-A addressing noise reduction and instrumental degradation issues, Atmos. Meas. Tech., 5, 2933-2949, doi: 10.5194/amt-5-2933-2012, 2012.

De Smedt, I., Theys, N., Yu, H., Danckaert, T., Lerot, C., Compernolle, S., Van Roozendael, M., Richter, A., Hilboll, A., Peters, E., Pedergnana, M., Loyola, D., Beirle, S., Wagner, T., Eskes, H., van Geffen, J., Boersma, K. F., and Veefkind, P.: Algorithm Theoretical Baseline for formaldehyde retrievals from S5P TROPOMI and from the QA4ECV project, Atmos. Meas. Tech. Discuss., 2017, 1-53, doi: 10.5194/amt-2017-393, 2017.

De Smedt, I., Theys, N., Yu, H., Danckaert, T., Lerot, C., Compernolle, S., Van Roozendael, M., Richter, A., Hilboll, A., Peters, E., Pedergnana, M., Loyola, D., Beirle, S., Wagner, T., Eskes, H., van Geffen, J., Boersma, K. F., and Veefkind, P.: Algorithm theoretical baseline for formaldehyde retrievals from S5P TROPOMI and from the QA4ECV project, Atmospheric Measurement Techniques, 11, 2395-2426, doi: 10.5194/amt-11-2395-2018, 2018.

de Zeeuw, M.: Quantifying $\mathrm{NO}_{x}$ emissions from Paris with high resolution satellite measurements M.Sc thesis, Wageningen University and Research, 2018.

Dee, D. P., Uppala, S. M., Simmons, A. J., Berrisford, P., Poli, P., Kobayashi, S., Andrae, U., Balmaseda, M. A., Balsamo, G., Bauer, P., Bechtold, P., Beljaars, A. C. M., van de Berg, L., Bidlot, J., Bormann, N., Delsol, C., Dragani, R., Fuentes, M., Geer, A. J., Haimberger, L., Healy, S. B., Hersbach, H., Hólm, E. V., Isaksen, L., Kållberg, P., Köhler, M., Matricardi, M., McNally, A. P., Monge-Sanz, B. M., Morcrette, J.-J., Park, B.-K., Peubey, C., de Rosnay, P., Tavolato, C., Thépaut, J.-N., and Vitart, F.: 
The ERA-Interim reanalysis: configuration and performance of the data assimilation system, Quarterly Journal of the Royal Meteorological Society, 137, 553-597, doi: 10.1002/qj.828, 2011.

Denier van der Gon, H. A. C., Visschedijk, A., Van der Brugh, H., and Dröge, R.: A high resolution European emission database for the year 2005, a contribution to the UBA-project PAREST: Particle Reduction Strategies, Tech. rep., tNO report TNO-034-UT-2010-01895-RPT-ML, 2010.

Deutschmann, T., Beirle, S., Frieß, U., Grzegorski, M., Kern, C., Kritten, L., Platt, U., Prados-Román, C., Pu,k ite, J., Wagner, T., Werner, B., and Pfeilsticker, K.: The Monte Carlo atmospheric radiative transfer model McArtim: Introduction and validation of Jacobians and 3D features, J. Quant. Spectrosc. Radiat. Transfer, 112, 1119 - 1137, doi: 10.1016/j.jqsrt.2010.12.009, 2011.

Dieudonné, E., Ravetta, F., Pelon, J., Goutail, F., and Pommereau, J.-P.: Linking $\mathrm{NO}_{2}$ surface concentration and integrated content in the urban developed atmospheric boundary layer, Geophysical Research Letters, 40, 1247-1251, doi: 10.1002/grl.50242, 2013.

Dirksen, R. J., Boersma, K. F., Eskes, H. J., Ionov, D. V., Bucsela, E. J., Levelt, P. F., and Kelder, H. M.: Evaluation of stratospheric $\mathrm{NO}_{2}$ retrieved from the Ozone Monitoring Instrument: Intercomparison, diurnal cycle, and trending, J. Geophys. Res.: Atmospheres, 116, n/a-n/a, doi: 10.1029/2010JD014943, d08305, 2011.

Duncan, B. N., Lamsal, L. N., Thompson, A. M., Yoshida, Y., Lu, Z., Streets, D. G., Hurwitz, M. M., and Pickering, K. E.: A space-based, high-resolution view of notable changes in urban $\mathrm{NO}_{x}$ pollution around the world (2005-2014), Journal of Geophysical Research: Atmospheres, 121, 976-996, doi: 10.1002/2015JD024121, 2016.

EEA: Air quality in Europe - 2016 Report, Tech. Rep. 28/2016, TH-AL-16-127-EN-N, European Environmental Agency (EEA), doi: 10.2800/80982, 2016.

EEA: France Air pollution fact sheet 2017, Tech. rep., European Environmental Agency (EEA), https://www.eea.europa.eu/themes/air/country-fact-sheets/france, 2017.

EEA: Air quality in Europe - 2017 Report, Tech. Rep. 13/2017, TH-AL-17-016-EN-N, European Environmental Agency (EEA), doi: doi:10.2800/850018, 2018.

Eskes, H. J. and Boersma, K. F.: Averaging kernels for DOAS total-column satellite retrievals, Atmospheric Chemistry and Physics, 3, 1285-1291, doi: 10.5194/acp-3-1285-2003, 2003.

Fangohr, S. and Kent, E. C.: An Estimate of Structural Uncertainty in QuikSCAT Wind Vector Retrievals, J. App. Meteo. and Clim., 51, 954-961, doi: 10.1175/JAMC-D-11-0183.1, 2012.

Fioletov, V. E., McLinden, C. A., Krotkov, N., Yang, K., Loyola, D. G., Valks, P., Theys, N., Van Roozendael, M., Nowlan, C. R., Chance, K., Liu, X., Lee, C., and Martin, R. V.: Application of OMI, SCIAMACHY, and GOME-2 satellite SO2 retrievals for detection of large emission sources, J. Geophys. Res.: Atmospheres, 118, 11,399-11,418, doi: 10.1002/jgrd.50826, 2013.

Fischer, P. H., Marra, M., Ameling, C. B., Hoek, G., Beelen, R., de Hoogh, K., Breugelmans, O., Kruize, H., Janssen, N. A., and Houthuijs, D.: Air Pollution and Mortality in Seven Million Adults: The Dutch Environmental Longitudinal Study (DUELS), Environmental health perspectives, 123(7), 697-704, doi: 10.1289/ehp.1408254, 2015.

Flemming, J., Huijnen, V., Arteta, J., Bechtold, P., Beljaars, A., Blechschmidt, A.-M., Diamantakis, M., Engelen, R. J., Gaudel, A., Inness, A., Jones, L., Josse, B., Katragkou, E., Marecal, V., Peuch, V.-H., Richter, A., Schultz, M. G., Stein, O., and Tsikerdekis, A.: Tropospheric chemistry in the Integrated Forecasting System of ECMWF, Geoscientific Model Development, 8, 975-1003, doi: 10.5194/gmd-8975-2015, 2015. 
Fu, D., Kulawik, S. S., Miyazaki, K., Bowman, K. W., Worden, J. R., Eldering, A., Livesey, N. J., Teixeira, J., Irion, F. W., Herman, R. L., Osterman, G. B., Liu, X., Levelt, P. F., Thompson, A. M., and Luo, M.: Retrievals of tropospheric ozone profiles from the synergism of AIRS and OMI: methodology and validation, Atmospheric Measurement Techniques, 11, 5587-5605, doi: 10.5194/amt-11-5587-2018, 2018.

Gao, F., Schaaf, C., Strahler, A., Jin, Y., and Li, X.: Detecting vegetation structure using a kernel-based BRDF model, Remote Sensing of Environment, 86, 198 - 205, doi: https://doi.org/10.1016/S00344257(03)00100-7, 2003.

Hales, S., Blakely, T., and Woodward, A.: Air pollution and mortality in New Zealand: cohort study, J. Epidemiol. Community Health, 66(5), 468-473, doi: 10.1136/jech.2010.112490, 2012.

Heckel, A., Kim, S.-W., Frost, G. J., Richter, A., Trainer, M., and Burrows, J. P.: Influence of low spatial resolution a priori data on tropospheric $\mathrm{NO}_{2}$ satellite retrievals, Atmos. Meas. Tech., 4, 1805-1820, doi: 10.5194/amt-4-1805-2011, 2011.

Hewson, W., Barkley, M. P., Gonzalez Abad, G., Bösch, H., Kurosu, T., Spurr, R., and Tilstra, L. G.: Development and characterisation of a state-of-the-art GOME-2 formaldehyde air-mass factor algorithm, Atmos. Meas. Tech., 8, 4055-4074, doi: 10.5194/amt-8-4055-2015, 2015.

Hilboll, A., Richter, A., Rozanov, A., Hodnebrog, Ø., Heckel, A., Solberg, S., Stordal, F., and Burrows, J. P.: Improvements to the retrieval of tropospheric $\mathrm{NO}_{2}$ from satellite - stratospheric correction using SCIAMACHY limb/nadir matching and comparison to Oslo CTM2 simulations, Atmos. Meas. Tech., 6, 565-584, doi: 10.5194/amt-6-565-2013, 2013.

Hu, H., Landgraf, J., Detmers, R., Borsdorff, T., Aan de Brugh, J., Aben, I., Butz, A., and Hasekamp, O.: Toward Global Mapping of Methane With TROPOMI: First Results and Intersatellite Comparison to GOSAT, Geophysical Research Letters, 45, 3682-3689, doi: 10.1002/2018GL077259, 2018.

Huang, M., Bowman, K. W., Carmichael, G. R., Lee, M., Chai, T., Spak, S. N., Henze, D. K., Darmenov, A. S., and da Silva, A. M.: Improved western U.S. background ozone estimates via constraining nonlocal and local source contributions using Aura TES and OMI observations, Journal of Geophysical Research: Atmospheres, 120, 3572-3592, doi: 10.1002/2014JD022993, 2015.

Inness, A., Baier, F., Benedetti, A., Bouarar, I., Chabrillat, S., Clark, H., Clerbaux, C., Coheur, P., Engelen, R. J., Errera, Q., Flemming, J., George, M., Granier, C., Hadji-Lazaro, J., Huijnen, V., Hurtmans, D., Jones, L., Kaiser, J. W., Kapsomenakis, J., Lefever, K., Leitão, J., Razinger, M., Richter, A., Schultz, M. G., Simmons, A. J., Suttie, M., Stein, O., Thépaut, J.-N., Thouret, V., Vrekoussis, M., Zerefos, C., and the MACC team: The MACC reanalysis: an 8 yr data set of atmospheric composition, Atmos. Chem. Phys., 13, 4073-4109, doi: 10.5194/acp-13-4073-2013, 2013.

Inness, A., Blechschmidt, A.-M., Bouarar, I., Chabrillat, S., Crepulja, M., Engelen, R. J., Eskes, H., Flemming, J., Gaudel, A., Hendrick, F., Huijnen, V., Jones, L., Kapsomenakis, J., Katragkou, E., Keppens, A., Langerock, B., de Mazière, M., Melas, D., Parrington, M., Peuch, V. H., Razinger, M., Richter, A., Schultz, M. G., Suttie, M., Thouret, V., Vrekoussis, M., Wagner, A., and Zerefos, C.: Data assimilation of satellite-retrieved ozone, carbon monoxide and nitrogen dioxide with ECMWF's Composition-IFS, Atmospheric Chemistry and Physics, 15, 5275-5303, doi: 10.5194/acp-15-5275-2015, 2015.

Irie, H., Kanaya, Y., Akimoto, H., Tanimoto, H., Wang, Z., Gleason, J. F., and Bucsela, E. J.: Validation of OMI tropospheric $\mathrm{NO}_{2}$ column data using MAX-DOAS measurements deep inside the North China Plain in June 2006: Mount Tai Experiment 2006, Atmos. Chem. Phys., 8, 6577-6586, doi: 10.5194/acp8-6577-2008, 2008. 
Jacob, D. J.: Introduction to Atmospheric Chemistry, Princeton Uni. Press, Pricneton. N. J., 1999.

Jacob, D. J., Horowitz, L. W., Munger, J. W., Heikes, B. G., Dickerson, R. R., Artz, R. S., and Keene, W. C.: Seasonal transition from NOx- to hydrocarbon-limited conditions for ozone production over the eastern United States in September, Journal of Geophysical Research: Atmospheres, 100, 9315-9324, doi: 10.1029/94JD03125, 1995.

Jiang, Z., McDonald, B. C., Worden, H., Worden, J. R., Miyazaki, K., Qu, Z., Henze, D. K., Jones, D. B. A., Arellano, A. F., Fischer, E. V., Zhu, L., and Boersma, K. F.: Unexpected slowdown of US pollutant emission reduction in the past decade, Proceedings of the National Academy of Sciences, 115, 5099-5104, doi: 10.1073/pnas.1801191115, 2018.

Jin, X., Fiore, A. M., Murray, L. T., Valin, L. C., Lamsal, L. N., Duncan, B., Boersma, K. F., De Smedt, I., Gonzalez-Abad, G., Chance, K., and Tonnesen, G. S.: Evaluating a Space-Based Indicator of Surface Ozone-NOx-VOC Sensitivity Over Midlatitude Source Regions and Application to Decadal Trends, Journal of Geophysical Research: Atmospheres, 122, 10,439-10,461, doi: 10.1002/2017JD026720, 2017.

Kleipool, Q. L., Dobber, M. R., de Haan, J. F., and Levelt, P. F.: Earth surface reflectance climatology from 3 years of OMI data, J. Geophys. Res.: Atmospheres, 113, doi: 10.1029/2008JD010290, d18308, 2008.

Koelemeijer, R. B. A., Stammes, P., Hovenier, J. W., and de Haan, J. F.: Global distributions of effective cloud fraction and cloud top pressure derived from oxygen A band spectra measured by the Global Ozone Monitoring Experiment: Comparison to ISCCP data, J. Geophys. Res.: Atmospheres, 107, AAC 5-1-AAC 5-9, doi: 10.1029/2001JD000840, 2002.

Koelemeijer, R. B. A., de Haan, J. F., and Stammes, P.: A database of spectral surface reflectivity in the range 335-772 $\mathrm{nm}$ derived from 5.5 years of GOME observations, J. Geophys. Res.: Atmospheres, 108, n/a-n/a, doi: 10.1029/2002JD002429, 4070, 2003.

Koepke, P.: Effective reflectance of oceanic whitecaps, Appl. Opt., 23, 1816-1824, doi: 10.1364/AO.23.001816, 1984.

Krotkov, N. A., McLinden, C. A., Li, C., Lamsal, L. N., Celarier, E. A., Marchenko, S. V., Swartz, W. H., Bucsela, E. J., Joiner, J., Duncan, B. N., Boersma, K. F., Veefkind, J. P., Levelt, P. F., Fioletov, V. E., Dickerson, R. R., He, H., Lu, Z., and Streets, D. G.: Aura OMI observations of regional $\mathrm{SO}_{2}$ and $\mathrm{NO}_{2}$ pollution changes from 2005 to 2015, Atmos. Chem. Phys., 16, 4605-4629, doi: 10.5194/acp-16-4605-2016, 2016.

Kuenen, J. J. P., Visschedijk, A. J. H., Jozwicka, M., and Denier van der Gon, H. A. C.: TNO-MACC/II emission inventory; a multi-year (2003-2009) consistent high-resolution European emission inventory for air quality modelling, Atmospheric Chemistry and Physics, 14, 10 963-10 976, doi: 10.5194/acp14-10963-2014, 2014.

Kuhlmann, G., Lam, Y. F., Cheung, H. M., Hartl, A., Fung, J. C. H., Chan, P. W., and Wenig, M. O.: Development of a custom $\mathrm{OMI} \mathrm{NO}_{2}$ data product for evaluating biases in a regional chemistry transport model, Atmos. Chem. Phys., 15, 5627-5644, doi: 10.5194/acp-15-5627-2015, 2015.

Lacis, A. A., Chowdhary, J., Mishchenko, M. I., and Cairns, B.: Modeling errors in diffuse sky radiation: Vector vs scalar treatment, Geophysical Research Letters, 25, 135-138, doi: 10.1029/97GL03613, 1998.

Lamsal, L. N., Martin, R. V., van Donkelaar, A., Steinbacher, M., Celarier, E. A., Bucsela, E., Dunlea, E. J., and Pinto, J. P.: Ground-level nitrogen dioxide concentrations inferred from the satellite-borne Ozone Monitoring Instrument, J. Geophys. Res.: Atmospheres, 113, doi: 10.1029/2007JD009235, d16308, 2008. 
Lamsal, L. N., Martin, R. V., Padmanabhan, A., van Donkelaar, A., Zhang, Q., Sioris, C. E., Chance, K., Kurosu, T. P., and Newchurch, M. J.: Application of satellite observations for timely updates to global anthropogenic NOx emission inventories, Geophysical Research Letters, 38, doi: 10.1029/2010GL046476, 2011.

Lamsal, L. N., Krotkov, N. A., Celarier, E. A., Swartz, W. H., Pickering, K. E., Bucsela, E. J., Gleason, J. F., Martin, R. V., Philip, S., Irie, H., Cede, A., Herman, J., Weinheimer, A., Szykman, J. J., and Knepp, T. N.: Evaluation of OMI operational standard $\mathrm{NO}_{2}$ column retrievals using in situ and surface-based $\mathrm{NO}_{2}$ observations, Atmos. Chem. Phys., 14, 11 587-11 609, doi: 10.5194/acp-14-11587$2014,2014$.

Laughner, J. L., Zare, A., and Cohen, R. C.: Effects of daily meteorology on the interpretation of spacebased remote sensing of $\mathrm{NO}_{2}$, Atmos. Chem. Phys. Discuss., 2016, 1-27, doi: 10.5194/acp-2016-536, 2016.

Leitao, J., Richter, A., Vrekoussis, M., Kokhanovsky, A., Zhang, Q. J., Beekmann, M., and Burrows, J. P.: On the improvement of $\mathrm{NO}_{2}$ satellite retrievals - aerosol impact on the airmass factors, Atmos. Meas. Tech., 3, 475-493, doi: 10.5194/amt-3-475-2010, 2010.

Lenoble, J.: Atmospheric Radiative Transfer, A. Deepak, Hampton, Va., 1993.

Levelt, P. F., Hilsenrath, E., Leppelmeier, G. W., van den Oord, G. H. J., Bhartia, P. K., Tamminen, J., de Haan, J. F., and Veefkind, J. P.: Science objectives of the ozone monitoring instrument, IEEE Transactions on Geoscience and Remote Sensing, 44, 1199-1208, doi: 10.1109/TGRS.2006.872336, 2006.

Levelt, P. F., Joiner, J., Tamminen, J., Veefkind, J. P., Bhartia, P. K., Stein Zweers, D. C., Duncan, B. N., Streets, D. G., Eskes, H., van der A, R., McLinden, C., Fioletov, V., Carn, S., de Laat, J., DeLand, M., Marchenko, S., McPeters, R., Ziemke, J., Fu, D., Liu, X., Pickering, K., Apituley, A., González Abad, G., Arola, A., Boersma, F., Chan Miller, C., Chance, K., de Graaf, M., Hakkarainen, J., Hassinen, S., Ialongo, I., Kleipool, Q., Krotkov, N., Li, C., Lamsal, L., Newman, P., Nowlan, C., Suleiman, R., Tilstra, L. G., Torres, O., Wang, H., and Wargan, K.: The Ozone Monitoring Instrument: overview of 14 years in space, Atmospheric Chemistry and Physics, 18, 5699-5745, doi: 10.5194/acp-18-5699-2018, 2018.

Li, C., Joiner, J., Krotkov, N. A., and Dunlap, L.: A new method for global retrievals of HCHO total columns from the Suomi National Polar-orbiting Partnership Ozone Mapping and Profiler Suite, Geophys. Res. Lett., 42, 2515-2522, doi: 10.1002/2015GL063204, 2015GL063204, 2015.

Li, X. and Strahler, A. H.: Geometric-Optical Bidirectional Reflectance Modeling of a Conifer Forest Canopy, IEEE Trans. Geo. Rem. Sens., GE-24, 906-919, doi: 10.1109/TGRS.1986.289706, 1986.

Lin, J.-T.: Satellite constraint for emissions of nitrogen oxides from anthropogenic, lightning and soil sources over East China on a high-resolution grid, Atmos. Chem. Phys., 12, 2881-2898, doi: 10.5194/acp-12-2881-2012, 2012.

Lin, J.-T., Martin, R. V., Boersma, K. F., Sneep, M., Stammes, P., Spurr, R., Wang, P., Van Roozendael, M., Clémer, K., and Irie, H.: Retrieving tropospheric nitrogen dioxide from the Ozone Monitoring Instrument: effects of aerosols, surface reflectance anisotropy, and vertical profile of nitrogen dioxide, Atmos. Chem. Phys., 14, 1441-1461, doi: 10.5194/acp-14-1441-2014, 2014.

Lin, J.-T., Liu, M.-Y., Xin, J.-Y., Boersma, K. F., Spurr, R., Martin, R., and Zhang, Q.: Influence of aerosols and surface reflectance on satellite $\mathrm{NO}_{2}$ retrieval: seasonal and spatial characteristics and implications for $\mathrm{NO}_{x}$ emission constraints, Atmos. Chem. Phys., 15, 11 217-11 241, doi: 10.5194/acp15-11217-2015, 2015. 
Litvinov, P., Hasekamp, O., Cairns, B., and Mishchenko, M. I.: Semi-empirical BRDF and BPDF models applied to the problem of aerosol retrievals over land: testing on airborne data and implications for modeling of top-of-atmosphere measurements, pp. 313-340, Springer Netherlands, doi: 10.1007/97894-007-1636-0_13, 2011.

Liu, M., Lin, J., Boersma, K. F., Pinardi, G., Wang, Y., Chimot, J., Wagner, T., Xie, P., Eskes, H., Van Roozendael, M., Hendrick, F., Wang, P., Wang, T., Yan, Y., Chen, L., and Ni, R.: Improved aerosol correction for OMI tropospheric $\mathrm{NO}_{2}$ retrieval over East Asia: constraint from CALIOP aerosol vertical profile, Atmospheric Measurement Techniques, 12, 1-21, doi: 10.5194/amt-12-1-2019, 2019.

Liu, W., Huang, B., Thorne, P. W., Banzon, V. F., Zhang, H.-M., Freeman, E., Lawrimore, J., Peterson, T. C., Smith, T. M., and Woodruff, S. D.: Extended Reconstructed Sea Surface Temperature Version 4 (ERSST.v4): Part II. Parametric and Structural Uncertainty Estimations, J. Climate, 28, 931-951, doi: 10.1175/JCLI-D-14-00007.1, 2015.

Lorente, A., Hilboll, A., Wang, Y., Dörner, S., Yu, H., and Boersma, K. F.: Air Mass Factor Calculation Best Practices and Recommendations, Sect. 2.5 of QA4ECV Deliverable 4.2 version 1.0, URL http://www.qa4ecv.eu/sites/default/files/D4.2.pdf, last access : June 2018, 2016.

Lorente, A., Boersma, K. F., Yu, H., Dörner, S., Hilboll, A., Richter, A., Liu, M., Lamsal, L. N., Barkley, M., De Smedt, I., Van Roozendael, M., Wang, Y., Wagner, T., Beirle, S., Lin, J.-T., Krotkov, N., Stammes, P., Wang, P., Eskes, H. J., and Krol, M.: Structural uncertainty in air mass factor calculation for $\mathrm{NO}_{2}$ and HCHO satellite retrievals, Atmos. Meas. Tech., 10, 759-782, doi: 10.5194/amt-10-759$2017,2017$.

Lorente, A., Boersma, K. F., Stammes, P., Tilstra, L. G., Richter, A., Yu, H., Kharbouche, S., and Muller, J. P.: The importance of surface reflectance anisotropy for cloud and $\mathrm{NO}_{2}$ retrievals from GOME-2 and OMI, Atmos. Meas. Tech., 11, 4509-4529, doi: 10.5194/amt-11-4509-2018, 2018.

Maasakkers, J.: Vital improvements to the retrieval of tropospheric $\mathrm{NO}_{2}$ columns from the Ozone Monitoring instrument, M.Sc thesis, Eindhoven University of Technology, 2013.

Maignan, F., Bréon, F.-M., and Lacaze, R.: Bidirectional reflectance of Earth targets: evaluation of analytical models using a large set of spaceborne measurements with emphasis on the Hot Spot, Remote Sensing of Environment, 90, 210 - 220, doi: http://doi.org/10.1016/j.rse.2003.12.006, 2004.

Marais, E. A., Jacob, D. J., Guenther, A., Chance, K., Kurosu, T. P., Murphy, J. G., Reeves, C. E., and Pye, H. O. T.: Improved model of isoprene emissions in Africa using Ozone Monitoring Instrument (OMI) satellite observations of formaldehyde: implications for oxidants and particulate matter, Atmos. Chem. Phys., 14, 7693-7703, doi: 10.5194/acp-14-7693-2014, 2014.

Marbach, T., Beirle, S., Platt, U., Hoor, P., Wittrock, F., Richter, A., Vrekoussis, M., Grzegorski, M., Burrows, J. P., and Wagner, T.: Satellite measurements of formaldehyde linked to shipping emissions, Atmos. Chem. Phys., 9, 8223-8234, doi: 10.5194/acp-9-8223-2009, 2009.

Martin, R. V., Jacob, D. J., Chance, K., Kurosu, T. P., Palmer, P. I., and Evans, M. J.: An improved retrieval of tropospheric nitrogen dioxide from GOME, J. Geophys. Res., 107, 4437, doi: 10.1029/2001JD001027, 2002.

Martin, R. V., Jacob, D. J., Chance, K.and Kurosu, T. P., Palmer, P. I., and Evans, M. J.: Global inventory of nitrogen oxide emissions constrained by space-based observations of NO2 columns, J. Geophys. Res.: Atmospheres, 108, doi: 10.1029/2003JD003453, URL http://dx.doi.org/10.1029/2003JD003453, 4537, 2003.

MCD43A1: MODIS/Terra and Aqua BRDF/Albedo Model Parameters Daily L3 Global $500 \mathrm{~m}$ SIN Grid V006, doi: DOI: 10.5067/MODIS/MCD43A1.006, URL 
https://ladsweb.nascom.nasa.gov/api/v1/productPage/product=MCD43A1.

McDonald, B. C., de Gouw, J. A., Gilman, J. B., Jathar, S. H., Akherati, A., Cappa, C. D., Jimenez, J. L., Lee-Taylor, J., Hayes, P. L., McKeen, S. A., Cui, Y. Y., Kim, S.-W., Gentner, D. R., IsaacmanVanWertz, G., Goldstein, A. H., Harley, R. A., Frost, G. J., Roberts, J. M., Ryerson, T. B., and Trainer, M.: Volatile chemical products emerging as largest petrochemical source of urban organic emissions, Science, 359, 760-764, doi: 10.1126/science.aaq0524, 2018.

McLinden, C. A., Fioletov, V., Boersma, K. F., Kharol, S. K., Krotkov, N., Lamsal, L., Makar, P. A., Martin, R. V., Veefkind, J. P., and Yang, K.: Improved satellite retrievals of $\mathrm{NO}_{2}$ and $\mathrm{SO}_{2}$ over the Canadian oil sands and comparisons with surface measurements, Atmos. Chem. Phys., 14, 3637-3656, doi: 10.5194/acp-14-3637-2014, 2014.

Mebust, A. K., Russell, A. R., Hudman, R. C., Valin, L. C., and Cohen, R. C.: Characterization of wildfire $\mathrm{NO}_{x}$ emissions using MODIS fire radiative power and OMI tropospheric $\mathrm{NO}_{2}$ columns, Atmospheric Chemistry and Physics, 11, 5839-5851, doi: 10.5194/acp-11-5839-2011, 2011.

Miyazaki, K., Eskes, H. J., Sudo, K., Takigawa, M., van Weele, M., and Boersma, K. F.: Simultaneous assimilation of satellite $\mathrm{NO}_{2}, \mathrm{O}_{3}, \mathrm{CO}$, and $\mathrm{HNO}_{3}$ data for the analysis of tropospheric chemical composition and emissions, Atmos. Chem. Phys., 12, 9545-9579, doi: 10.5194/acp-12-9545-2012, 2012.

Miyazaki, K., Eskes, H., Sudo, K., Boersma, K. F., Bowman, K., and Kanaya, Y.: Decadal changes in global surface $\mathrm{NO}_{x}$ emissions from multi-constituent satellite data assimilation, Atmospheric Chemistry and Physics, 17, 807-837, doi: 10.5194/acp-17-807-2017, 2017.

Miyazaki, K., Sekiya, T., Fu, D., Bowman, K. W., Kulawik, S. S., Sudo, K., Walker, T., Kanaya, Y., Takigawa, M., Ogochi, K., Eskes, H., Boersma, K. F., Thompson, A. M., Gaubert, B., Barre, J., and Emmons, L. K.: Balance of Emission and Dynamical Controls on Ozone During the KoreaUnited States Air Quality Campaign From Multiconstituent Satellite Data Assimilation, Journal of Geophysical Research: Atmospheres, 124, doi: 10.1029/2018JD028912, 2018.

Morel, A.: Optical modeling of the upper ocean in relation to its biogenous matter content (case I waters), Journal of Geophysical Research: Oceans, 93, 10 749-10 768, doi: 10.1029/JC093iC09p10749, 1988.

Müller, J.-P., Kharbouche, S., Gobron, N., Scanlon, T., Govaerts, Y., Danne, O., Schultz, J., Lattanzio, A., Peters, E., De Smedt, I., Beirle, S., Lorente, A., Coheur, P. F., George, M., Wagner, T., Hilboll, A., Richter, A., Van Roozendael, M., and Boersma, K. F.: Recommendations (scientific) on best practices for retrievals for Land and Atmosphere ECVs (QA4ECV Deliverable 4.2 version 1.0), URL http://www.qa4ecv.eu/sites/default/files/D4.2.pdf, last access : June 2018, 2016.

Munro, R., Lang, R., Klaes, D., Poli, G., Retscher, C., Lindstrot, R., Huckle, R., Lacan, A., Grzegorski, M., Holdak, A., Kokhanovsky, A., Livschitz, J., and Eisinger, M.: The GOME-2 instrument on the Metop series of satellites: instrument design, calibration, and level 1 data processing - an overview, Atmos. Meas. Tech., 9, 1279-1301, doi: 10.5194/amt-9-1279-2016, 2016.

Myhre, G., Kvalevåg, M. M., and Schaaf, C. B.: Radiative forcing due to anthropogenic vegetation change based on MODIS surface albedo data, Geophysical Research Letters, 32, n/a-n/a, doi: 10.1029/2005GL024004, 121410, 2005.

Myhre, G., Shindell, D., Bréon, F.-M., Collins, W., Fuglestvedt, J., Huang, J., Koch, D., Lamarque, J.-F., Lee, D., Mendoza, B., Nakajima, T., Robock, A., Stephens, G., Takemura, T., and Zhang, H.: Climate Change 2013: The Physical Science Basis. Contribution of Working Group I to the Fifth Assessment Report of the Intergovernmental Panel on Climate Change, Cambridge University Press, Cambridge, United Kingdom and New York, NY, USA, doi: 10.1017/CBO9781107415324, URL www. climatechange2013.org, 2013. 
Naik, V., Mauzerall, D., Horowitz, L., Schwarzkopf, M. D., Ramaswamy, V., and Oppenheimer, M.: Net radiative forcing due to changes in regional emissions of tropospheric ozone precursors, Journal of Geophysical Research: Atmospheres, 110, doi: 10.1029/2005JD005908, 2005.

Nicodemus, F. E., Richmond, J. C., Hsia, J. J., Ginsberg, I. W., and Limperis, T.: in: Radiometry, edited by Wolff, L. B., Shafer, S. A., and Healey, G., chap. Geometrical Considerations and Nomenclature for Reflectance, pp. 94-145, Jones and Bartlett Publishers, Inc., USA, 1992.

Noguchi, K., Richter, A., Rozanov, V., Rozanov, A., Burrows, J. P., Irie, H., and Kita, K.: Effect of surface BRDF of various land cover types on geostationary observations of tropospheric $\mathrm{NO}_{2}$, Atmos. Meas. Tech., 7, 3497-3508, doi: 10.5194/amt-7-3497-2014, 2014.

NRC: Climate Data Records from Environmental Satellites, Tech. rep., National Research Council, Washington D.C., 2004.

Oldeman, A.: Effect of including an intensity offset in the DOAS $\mathrm{NO}_{2}$ retrieval of TROPOMI, Internship report, Eindhoven University of Technology/R-D Satellite Observations, KNMI, 2018.

Oleson, K. W., Bonan, G. B., Schaaf, C., Gao, F., Jin, Y., and Strahler, A.: Assessment of global climate model land surface albedo using MODIS data, Geophysical Research Letters, 30, n/a-n/a, doi: 10.1029/2002GL016749, 1443, 2003.

O'Hirok, W. and Gautier, C.: A Three-Dimensional Radiative Transfer Model to Investigate the Solar Radiation within a Cloudy Atmosphere. Part I: Spatial Effects, J. Atmos. Sci., 55, 2162-2179, doi: 10.1175/1520-0469(1998)055<2162:ATDRTM>2.0.CO;2, 1998.

Palmer, P. I., Jacob, D. J., Chance, K., Martin, R. V., Spurr, R. J. D., Kurosu, T. P., Bey, I., Yantosca, R., Fiore, A., and Li, Q.: Air mass factor formulation for spectroscopic measurements from satellites: Application to formaldehyde retrievals from the Global Ozone Monitoring Experiment, J. Geophys. Res., 106, 14539-14 550, 2001.

Peters, E., Wittrock, F., Richter, A., Alvarado, L. M. A., Rozanov, V. V., and Burrows, J. P.: Liquid water absorption and scattering effects in DOAS retrievals over oceans, Atmos. Meas. Tech., 7, 42034221, doi: 10.5194/amt-7-4203-2014, 2014.

Peters, E., De Smedt, I., , and Van Geffen, J.: Spectral Fitting Best Practices and Recommendations, Sect. 2.3 of QA4ECV Deliverable 4.2 version 1.0, URL http://www.qa4ecv.eu/sites/default/files/D4.2.pdf, last access : June 2018, 2016.

Petetin, H., Beekmann, M., Colomb, A., Denier van der Gon, H. A. C., Dupont, J.-C., Honoré, C., Michoud, V., Morille, Y., Perrussel, O., Schwarzenboeck, A., Sciare, J., Wiedensohler, A., and Zhang, Q. J.: Evaluating $\mathrm{BC}$ and $\mathrm{NO}_{x}$ emission inventories for the Paris region from MEGAPOLI aircraft measurements, Atmospheric Chemistry and Physics, 15, 9799-9818, doi: 10.5194/acp-15-9799-2015, 2015 .

Platt, U.: Air Monitoring by Differential Optical Absorption Spectroscopy, pp. 1-28, American Cancer Society, doi: 10.1002/9780470027318.a0706.pub2, 2017.

Povey, A. C. and Grainger, R. G.: Known and unknown unknowns: uncertainty estimation in satellite remote sensing, Atmos. Meas. Tech., 8, 4699-4718, doi: 10.5194/amt-8-4699-2015, 2015.

QA4ECV-WP4: Product User Guide for Land ECVs and Product Specification Document for Atmosphere ECV precursors. Part 1: Product User Guide for QA4ECV-albedo, Deliverable 4.6, 2016.

Querol, X., Alastuey, A., Gangoiti, G., Perez, N., Lee, H. K., Eun, H. R., Park, Y., Mantilla, E., Escudero, M., Titos, G., Alonso, L., Temime-Roussel, B., Marchand, N., Moreta, J. R., Revuelta, M. A., Salvador, P., Artíñano, B., García dos Santos, S., Anguas, M., Notario, A., Saiz-Lopez, A., 
Harrison, R. M., Millán, M., and Ahn, K.-H.: Phenomenology of summer ozone episodes over the Madrid Metropolitan Area, central Spain, Atmospheric Chemistry and Physics, 18, 6511-6533, doi: 10.5194/acp-18-6511-2018, 2018.

Ren, X., Harder, H., Martinez, M., Lesher, R. L., Oliger, A., Simpas, J. B., Brune, W. H., Schwab, J. J., Demerjian, K. L., He, Y., Zhou, X., and Gao, H.: OH and HO2 Chemistry in the urban atmosphere of New York City, Atmospheric Environment, 37, 3639 - 3651, doi: https://doi.org/10.1016/S13522310(03)00459-X, 2003.

Richter, A.: Absorptionsspektroskopische Messungen stratosphärischer Spurengase über Bremen, 53 N,, Ph.D. thesis, University of Bremen, 1997.

Richter, A. and Burrows, J.: Tropospheric $\mathrm{NO}_{2}$ from GOME measurements, Adv. Space Res., 29", 1673 - 1683, doi: http://dx.doi.org/10.1016/S0273-1177(02)00100-X, 2002.

Richter, A., Burrows, J. P., Nusz, H., Granier, C., and Niemeier, U.: Increase in tropospheric nitrogen dioxide over China observed from space, Nature, 437, 129-132, doi: 10.1038/nature04092, 2005.

Richter, A., Begoin, M., Hilboll, A., and Burrows, J. P.: An improved $\mathrm{NO}_{2}$ retrieval for the GOME-2 satellite instrument, Atmos. Meas. Tech., 4, 1147-1159, doi: 10.5194/amt-4-1147-2011, 2011.

Roujean, J.-L., Leroy, M., and Deschamps, P.-Y.: A bidirectional reflectance model of the Earth's surface for the correction of remote sensing data, J. Geophys. Res., 97, 20 455-20 468, doi: 10.1029/92JD01411, 1992.

Rozanov, V. V., Rozanov, A. V., Kokhanovsky, A. A., and Burrows, J. P.: Radiative tranfer through terrestrial atmosphere and ocean: Software package SCIATRAN, J. Quant. Spectrosc. Radiat. Transfer, 133, 13-71, doi: 10.1016/j.jqsrt.2013.07.004, 2014.

Russell, A. R., Perring, A. E., Valin, L. C., Bucsela, E. J., Browne, E. C., Wooldridge, P. J., and Cohen, R. C.: A high spatial resolution retrieval of $\mathrm{NO}_{2}$ column densities from OMI: method and evaluation, Atmos. Chem. Phys., 11, 8543-8554, doi: 10.5194/acp-11-8543-2011, 2011.

S5P Verification Team: Sentinel-5P TROPOMI Science Verification Report, Issue 2.1, S5P-IUP-L2-ScVRRP, Technical Document, 314 pp, https://earth.esa.int/web/sentinel/user-guides/sentinel-5p-tropomi, last access on 5 September 2018, 2015.

Saiz-Lopez, A., Borge, R., Notario, A., Adame, J. A., De la Paz, D., Querol, X., Artíñano, B., GomezMoreno, F. J., and Cuevas, C. A.: Unexpected increase in the oxidation capacity of the urban atmosphere of Madrid, Spain, Scientific Reports, doi: https://doi.org/10.1038/srep45956, 2017.

Schaepman-Strub, G., Schaepman, M., Painter, T., Dangel, S., and Martonchik, J.: Reflectance quantities in optical remote sensing - definitions and case studies, Remote Sensing of Environment, 103, 27 - 42 , doi: https://doi.org/10.1016/j.rse.2006.03.002, 2006.

Schaub, D., Boersma, K. F., Kaiser, J. W., Weiss, A. K., Folini, D., Eskes, H. J., and Buchmann, B.: Comparison of GOME tropospheric $\mathrm{NO}_{2}$ columns with $\mathrm{NO}_{2}$ profiles deduced from ground-based in situ measurements, Atmospheric Chemistry and Physics, 6, 3211-3229, doi: 10.5194/acp-6-3211-2006, 2006.

Shaiganfar, R., Beirle, S., Denier van der Gon, H., Jonkers, S., Kuenen, J., Petetin, H., Zhang, Q., Beekmann, M., and Wagner, T.: Estimation of the Paris $\mathrm{NO}_{x}$ emissions from mobile MAX-DOAS observations and CHIMERE model simulations during the MEGAPOLI campaign using the closed integral method, Atmos. Chem. and Phys., 17, 7853-7890, doi: 10.5194/acp-17-7853-2017, 2017.

Shindell, D. T., Faluvegi, G., Koch, D. M., Schmidt, G. A., Unger, N., and Bauer, S. E.: Improved Attribution of Climate Forcing to Emissions, Science, 326, 716-718, doi: 10.1126/science.1174760, 
2009.

Sindelarova, K., Granier, C., Bouarar, I., Guenther, A., Tilmes, S., Stavrakou, T., Müller, J.-F., Kuhn, U., Stefani, P., and Knorr, W.: Global data set of biogenic VOC emissions calculated by the MEGAN model over the last 30 years, Atmospheric Chemistry and Physics, 14, 9317-9341, doi: 10.5194/acp14-9317-2014, 2014.

Solomon, S., Portmann, R. W., Sanders, R. W., Daniel, J. S., Madsen, W., Bartram, B., and Dutton, E. G.: On the role of nitrogen dioxide in the absorption of solar radiation, Journal of Geophysical Research: Atmospheres, 104, 12 047-12 058, doi: 10.1029/1999JD900035, 1999.

Spurr, R. J. D.: A new approach to the retrieval of surface properties from earthshine measurements, J. Quant. Spectrosc. Radiat. Transfer, 83, 15 - 46, doi: http://doi.org/10.1016/S0022-4073(02)00283-2, 2004.

Spurr, R. J. D., Kurosu, T. P., and Chance, K. V.: A linearized discrete ordinate radiative transfer model for atmospheric remote-sensing retrieval, J. Quant. Spectrosc. Radiat. Transfer, 68, 689-735, 2001.

Stammes, P.: Spectral radiance modeling in the UV-Visible range, IRS2000: in: Current problems in atmospheric radiation, edited by: Smith, W. L. and Timofeyev, Y. J.,, pp. 385-388, 2001.

Stammes, P., de Haan, J. F., and Hovenier, J. W.: The polarized internal radiation field of a planetary atmosphere, Astron. Astrophys., 225, 239-259, 1989.

Stammes, P., Sneep, M., de Haan, J. F., Veefkind, J. P., Wang, P., and Levelt, P. F.: Effective cloud fractions from the Ozone Monitoring Instrument: Theoretical framework and validation, J. Geophys. Res.: Atmospheres, 113, n/a-n/a, doi: 10.1029/2007JD008820, d16S38, 2008.

Stavrakou, T., Müller, J.-F., Boersma, K. F., van der A, R. J., Kurokawa, J., Ohara, T., and Zhang, Q.: Key chemical $\mathrm{NO}_{x}$ sink uncertainties and how they influence top-down emissions of nitrogen oxides, Atmos. Chem. Phys., 13, 9057-9082, doi: 10.5194/acp-13-9057-2013, 2013.

Steiner, A. K., Hunt, D., Ho, S.-P., Kirchengast, G., Mannucci, A. J., Scherllin-Pirscher, B., Gleisner, H., von Engeln, A., Schmidt, T., Ao, C., Leroy, S. S., Kursinski, E. R., Foelsche, U., Gorbunov, M., Heise, S., Kuo, Y.-H., Lauritsen, K. B., Marquardt, C., Rocken, C., Schreiner, W., Sokolovskiy, S., Syndergaard, S., and Wickert, J.: Quantification of structural uncertainty in climate data records from GPS radio occultation, Atm. Chem. Phys., 13, 1469-1484, doi: 10.5194/acp-13-1469-2013, 2013.

Stevenson, D. S., Dentener, F. J., Schultz, M. G., Ellingsen, K., van Noije, T. P. C., Wild, O., Zeng, G., Amann, M., Atherton, C. S., Bell, N., Bergmann, D. J., Bey, I., Butler, T., Cofala, J., Collins, W. J., Derwent, R. G., Doherty, R. M., Drevet, J., Eskes, H. J., Fiore, A. M., Gauss, M., Hauglustaine, D. A., Horowitz, L. W., Isaksen, I. S. A., Krol, M. C., Lamarque, J.-F., Lawrence, M. G., Montanaro, V., Müller, J.-F., Pitari, G., Prather, M. J., Pyle, J. A., Rast, S., Rodriguez, J. M., Sanderson, M. G., Savage, N. H., Shindell, D. T., Strahan, S. E., Sudo, K., and Szopa, S.: Multimodel ensemble simulations of present-day and near-future tropospheric ozone, J. Geophys. Res.: Atmospheres, 111, doi: 10.1029/2005JD006338, 2006.

Strahler, A. H., Lucht, W., Schaaf, C. B., Tsang, T., Gao, F., Li X.and Muller, J. P., Lewis, P., and Barnsley, M. J.: MODIS BRDF/Albedo Product: Algorithm Theoretical Basis Document Version 5.0. MODIS Product ID: MOD43, 1999.

Tebaldi, C. and Knutti, R.: The use of the multi-model ensemble in probabilistic climate projections, Philosophical Transactions of the Royal Society of London A: Mathematical, Physical and Engineering Sciences, 365, 2053-2075, doi: 10.1098/rsta.2007.2076, 2007.

Thorne, P. W., Parker, D. E., Christy, J. R., and Mears, C. A.: Uncertainties in climate trends: Lessons 
from Upper-Air Temperature Records, Bull. Am. Meteorol. Soc., 86, 1437-1442, doi: 10.1175/BAMS86-10-1437, 2005.

Tilstra, L. G., Tuinder, O. N. E., Wang, P., and Stammes, P.: Surface reflectivity climatologies from UV to NIR determined from Earth observations by GOME-2 and SCIAMACHY, J. Geophys. Res.: Atmospheres, 122, 4084-4111, doi: 10.1002/2016JD025940, 2016JD025940, 2017.

Torres, O., Tanskanen, A., Veihelmann, B., Ahn, C., Braak, R., Bhartia, P. K., Veefkind, P., and Levelt, P.: Aerosols and surface UV products from Ozone Monitoring Instrument observations: An overview, J. Geophys. Res.: Atmospheres, 112, doi: 10.1029/2007JD008809, d24S47, 2007.

Valin, L. C., Russell, A. R., and Cohen, R. C.: Variations of OH radical in an urban plume inferred from NO2 column measurements, Geophysical Research Letters, 40, 1856-1860, doi: 10.1002/grl.50267, 2013.

Valks, P., Pinardi, G., Richter, A., Lambert, J.-C., Hao, N., Loyola, D., Van Roozendael, M., and Emmadi, S.: Operational total and tropospheric $\mathrm{NO}_{2}$ column retrieval for GOME2, Atmospheric Measurement Techniques, 4, 1491-1514, doi: 10.5194/amt-4-1491-2011, URL https://www . atmos-meas-tech.net/4/1491/2011/, 2011.

van der A, R. J., Eskes, H. J., Boersma, K. F., van Noije, T. P. C., Van Roozendael, M., De Smedt, I., Peters, D. H. M. U., and Meijer, E. W.: Trends, seasonal variability and dominant $\mathrm{NO}_{x}$ source derived from a ten year record of $\mathrm{NO}_{2}$ measured from space, Journal of Geophysical Research: Atmospheres, 113, doi: 10.1029/2007JD009021, 2008.

van Geffen, J. H. G. M., Boersma, K. F., Van Roozendael, M., Hendrick, F., Mahieu, E., De Smedt, I., Sneep, M., and Veefkind, J. P.: Improved spectral fitting of nitrogen dioxide from OMI in the 405-465 nm window, Atmos. Meas. Tech., 8, 1685-1699, doi: 10.5194/amt-8-1685-2015, 2015.

van Geffen, J. H. G. M., Eskes, H. J., Boersma, K. F., Maasakkers, J. D., and Veefkind, J. P.: TROPOMI ATBD of the total and tropospheric $\mathrm{NO}_{2}$ data products, S5P-KNMI-L2-0005-RP, issue 1.2.0, 2018.

Van Noije, T. P. C., Eskes, H. J., Dentener, F. J., Stevenson, D. S., Ellingsen, K., Schultz, M. G., Wild, O., Amann, M., Atherton, C. S., Bergmann, D. J., Bey, I., Boersma, K. F., Butler, T., Cofala, J., Drevet, J., Fiore, A. M., Gauss, M., Hauglustaine, D. A., Horowitz, L. W., Isaksen, I. S. A., Krol, M. C., Lamarque, J.-F., Lawrence, M. G., Martin, R. V., Montanaro, V., Müller, J.-F., Pitari, G., Prather, M. J., Pyle, J. A., Richter, A., Rodriguez, J. M., Savage, N. H., Strahan, S. E., Sudo, K., Szopa, S., and van Roozendael, M.: Multi-model ensemble simulations of tropospheric $\mathrm{NO}_{2}$ compared with GOME retrievals for the year 2000, Atmos. Chem. Phys., 6, 2943-2979, doi: 10.5194/acp-6-2943-2006, 2006.

Vandaele, A. C., Hermans, C., Fally, S., Carleer, M., Colin, R., Mérienne, M.-F., Jenouvrier, A., and Coquart, B.: High-resolution Fourier transform measurement of the $\mathrm{NO}_{2}$ visible and near-infrared absorption cross sections: Temperature and pressure effects, J. Geophys. Res.: Atmospheres, 107, ACH 3-1-ACH 3-12, doi: 10.1029/2001JD000971, 4348, 2002.

Vasilkov, A., Qin, W., Krotkov, N., Lamsal, L., Spurr, R., Haffner, D., Joiner, J., Yang, E.-S., and Marchenko, S.: Accounting for the effects of surface BRDF on satellite cloud and trace-gas retrievals: a new approach based on geometry-dependent Lambertian equivalent reflectivity applied to OMI algorithms, Atmos. Meas. Tech., 10, 333-349, doi: 10.5194/amt-10-333-2017, 2017.

Vaughan, G., Quinn, P. T., Green, A. C., Bean, J., Roscoe, H. K., van Roozendael, M., and Goutail, F.: SAOZ measurements of $\mathrm{NO}_{2}$ at Aberystwyth, J. Environ. Monit., 8, 353-361, doi: 10.1039/B511482A, 2006.

Veefkind, J., Aben, I., McMullan, K., Förster, H., de Vries, J., Otter, G., Claas, J., Eskes, H. J., 
de Haan, J. F., Kleipool, Q., van Weele, M., Hasekamp, O., Hoogeveen, R., Landgraf, J., Snel, R., Tol, P., Ingmann, P., Voors, R., Kruizinga, B., Vink, R., Visser, H., and Levelt, P.: TROPOMI on the ESA Sentinel-5 Precursor: A GMES mission for global observations of the atmospheric composition for climate, air quality and ozone layer applications, Remote Sensing of Environment, 120, 70 - 83, doi: https://doi.org/10.1016/j.rse.2011.09.027, the Sentinel Missions - New Opportunities for Science, 2012.

Veefkind, J. P., de Haan, J. F., Sneep, M., and Levelt, P. F.: Improvements to the $\mathrm{OMI} \mathrm{O}_{2}-\mathrm{O}_{2}$ operational cloud algorithm and comparisons with ground-based radar-lidar observations, Atmos. Meas. Tech., 9, 6035-6049, doi: 10.5194/amt-9-6035-2016, 2016.

Venstusky: Wind speef maps, Online, https://www.ventusky.com, 2018.

Vermote, E. F. and Kotchenova, S.: Atmospheric correction for the monitoring of land surfaces, J. Geophys. Res.: Atmospheres, 113, n/a-n/a, doi: 10.1029/2007JD009662, d23S90, 2008.

Vermote, E. F., El Saleous, N., Justice, C. O., Kaufman, Y. J., Privette, J. L., Remer, L., Roger, J. C., and Tanré, D.: Atmospheric correction of visible to middle-infrared EOS-MODIS data over land surfaces: Background, operational algorithm and validation, J. Geophys. Res.: Atmospheres, 102, 17 131-17141, doi: 10.1029/97JD00201, URL http://dx.doi.org/10.1029/97JD00201, 1997.

Verstraeten, W. W., Neu, J. L., Williams, J. E., Bowman, K. W., Worden, J. R., and Boersma, K. F.: Rapid increases in tropospheric ozone production and export from China, Nature Geoscience, pp. 690-695, 2015.

Vilà-Guerau de Arellano, J., van Heerwaarden, C. C., van Stratum, B. J., and an den Dries, K.: Atmospheric Boundary Layer: Integrating Air Chemistry and Land Interactions, Cambridge University Press, Cambridge, doi: doi:10.1017/CBO9781316117422, 2005.

Vinken, G. C. M., Boersma, K. F., van Donkelaar, A., and Zhang, L.: Constraints on ship $\mathrm{NO}_{x}$ emissions in Europe using GEOS-Chem and OMI satellite $\mathrm{NO}_{2}$ observations, Atmos. Chem. Phys., 14, 13531369, doi: 10.5194/acp-14-1353-2014, 2014.

Wagner, T., Burrows, J. P., Deutschmann, T., Dix, B., von Friedeburg, C., Frieß, U., Hendrick, F., Heue, K.-P., Irie, H., Iwabuchi, H., Kanaya, Y., Keller, J., McLinden, C. A., Oetjen, H., Palazzi, E., Petritoli, A., Platt, U., Postylyakov, O., Pukite, J., Richter, A., van Roozendael, M., Rozanov, A., Rozanov, V., Sinreich, R., Sanghavi, S., and Wittrock, F.: Comparison of box-air-mass-factors and radiances for Multiple-Axis Differential Optical Absorption Spectroscopy (MAX-DOAS) geometries calculated from different UV/visible radiative transfer models, Atmos. Chem. Phys., 7, 1809-1833, doi: 10.5194/acp-7-1809-2007, 2007.

Wang, P., Stammes, P., van der A, R., Pinardi, G., and van Roozendael, M.: FRESCO+: an improved $\mathrm{O}_{2}$ A-band cloud retrieval algorithm for tropospheric trace gas retrievals, Atmos. Chem. Phys., 8, 6565-6576, doi: 10.5194/acp-8-6565-2008, 2008.

Wanner, W., Li, X., and Strahler, A. H.: On the derivation of kernels for kernel-driven models of bidirectional reflectance, J. Geophys. Res., 100, 21 077-21 089, doi: 10.1029/95JD02371, 1995.

Williams, J. E., Boersma, K. F., Le Sager, P., and Verstraeten, W. W.: The high-resolution version of TM5-MP for optimized satellite retrievals: description and validation, Geoscientific Model Development, 10, 721-750, doi: 10.5194/gmd-10-721-2017, 2017.

Zara, M., Boersma, K. F., De Smedt, I., Richter, A., Peters, E., Van Geffen, J. H. G. M., Beirle, S., Wagner, T., Van Roozendael, M., Marchenko, S., Lamsal, L. N., and Eskes, H. J.: Improved slant column density retrieval of nitrogen dioxide and formaldehyde for OMI and GOME-2A from QA4ECV: intercomparison, uncertainty characterization, and trends, Atmospheric Measurement Techniques Dis- 
cussions, 2018, 1-47, doi: 10.5194/amt-2017-453, 2018.

Zhou, Y., Brunner, D., Boersma, K. F., Dirksen, R., and Wang, P.: An improved tropospheric $\mathrm{NO}_{2}$ retrieval for OMI observations in the vicinity of mountainous terrain, Atmospheric Measurement Techniques, 2, 401-416, doi: 10.5194/amt-2-401-2009, 2009.

Zhou, Y., Brunner, D., Spurr, R. J. D., Boersma, K. F., Sneep, M., Popp, C., and Buchmann, B.: Accounting for surface reflectance anisotropy in satellite retrievals of tropospheric $\mathrm{NO}_{2}$, Atmos. Meas. Tech., 3, 1185-1203, doi: 10.5194/amt-3-1185-2010, 2010.

Zhu, L., Mickley, L. J., Jacob, D. J., Marais, E. A., Sheng, J., Hu, L., Abad, G. G., and Chance, K.: Long-term (2005-2014) trends in formaldehyde (HCHO) columns across North America as seen by the OMI satellite instrument: Evidence of changing emissions of volatile organic compounds, Geophysical Research Letters, 44, 7079-7086, doi: 10.1002/2017GL073859, 2017. 


\section{Acknowledgements}

My first day as a PhD student, Folkert gave me a paper titled "The adding method for multiple scattering calculations of polarized light" (de Haan, 1987), which increased the steepness of my PhD learning curve quite a bit. Since then, every day I have learned something new both scientifically and socially, thanks to the people that have invested some time in me. I was definitely not born with any of the knowledge about atmospheric radiative transfer and satellites reflected in this thesis.

I would like to start thanking the two scientists that gave me the opportunity to start my scientific career and have guided me through this journey. Folkert, thank you for everything. For your guidance, the confidence and trust, for all the things that I have learnt from you both at professional and personal level, as these have shaped me to be the scientist that I am today. I have learnt from you how to write, how to present with impact, as well as how to be critical with my own work and with others. Thank you for all the things that you have taught me about satellites, radiative transfer and $\mathrm{NO}_{2}$. Your enthusiasm and love for science is an inspiration for me. Working with you has been a pleasure, and I hope that in the future our scientific paths will cross again.

Maarten, it has also been a pleasure to work with you, as you are always there to discuss science or to try to solve an impossible mathematical equation. I have learned a lot from you, not only about atmospheric chemistry but also about how to apply the scientific method, how to make science. Thanks also for the support in the last months of the $\mathrm{PhD}$, for your guidance and help in polishing the last pieces of this book.

Together with de Hann (1987), the first day at the office I also got the code of the radiative transfer model DAK. This has been a very important tool in my research, and what I have achieved with it would not have been possible without the support from Piet. Thanks Piet for guiding me through the infinite lines of code, and for teaching me how to translate those lines to physics. Thanks to you and Folkert, I can understand many of the things that Nature does with light and make science with it.

I am very grateful that I have been part of the MAQ group, and I feel satisfied when colleagues at MAQ smile when they hear the term "air mass factor" because they have learned what it is (or at least they have tried to). Thanks Wouter for organizing the air quality group and its meetings and nice uitjes, but most importantly thanks for increasing 
the women to men ratio of the department. This brings me to Ingrid, a reference for all the young women at MAQ, always there for a question and always there with an answer. Jordi, gracias por darle el toque sureño al departamento y por enseñar a los holandeses que comer es algo más que una ingesta de nutrientes. Tu sinceridad ante cualquier problema y tu manera de afrontar las cosas me han enseñado mucho. Tu implicación con los estudiantes y tu forma de hacer ciencia es una inspiración para todos y mantienen el espíritu de la universidad en su estado puro. To all the scientists at MAQ, thanks to you I have learned a lot about many scientific topics and about how to do science. The way science is done at MAQ is an inspiration, so keep doing outstanding science as you do and transmitting that to younger generations. Because without science, there is no future.

And this brings me to my beginnings when "one apple a day keeps the doctor away" by Eduardo and loud laughs by Marie were part of the routine in the office. Thanks to the "older PhD generation" for introducing me to the MAQ life, for all the nice moments together and for showing me that it is possible to finish a $\mathrm{PhD}$. But you guys left and some others joined the MAQ party committee. Thanks to the "new PhD generation" for introducing new traditions to MAQ that made our group to have enjoyable moments outside the office. I would like to give a special thank to Imme and Anna-Lena, for the big support (together with beers) in the last stretch of the $\mathrm{PhD}$.

During my PhD I have had a second office at KNMI, where OMI and TROPOMI are the centre of the universe and "air mass factor" is a simple term. Thanks to all the scientists that generously introduced me to the remote sensing world and have shared their knowledge with me. I would like to thank particularly Henk, Jos, and Ping, for always being willing to explain anything to me, for the help and support. Marina, thanks for always responding to any request from my side, and for bringing fun and laughs to KNMI. I have also been lucky to be part of a project (QA4ECV, read the thesis!) where I have learned a lot from a group of great scientists, to whom I am thankful for the support that I have received during my PhD.

I could have definitely had 10 paranimphs instead of 2. Living in Wageningen for four years have brought many many incredible people into my life, some of which are part of my family now, and for this I am very very grateful to life. People say that the Spanish tend to get together when they are abroad, and that's true. But I have learned that "Spanish" can be extended to many other countries when the people you meet have a big heart. Thanks to my Elisa's fan club family in Wageningen, for all the laughs and good moments, the food, the home made beer, the wine, the endless conversations, for the hugs, and for this lifetime "Iamezing" friendship that I will take with me wherever I go. To my beloved Brazilians, thanks for your warmth, kindness and for teaching me many things about life. To the extended Brazilians and all other friends of many nationalities, thanks for making me enjoy life in Wageningen. For many many more saideras more! Since I moved to Utrecht, I have been warmly welcomed by an amazing group of friends that are part of my life now; thanks Vecinos for many wonderful moments. I would also 
like to thank the Pluto team for the enjoyable basketball time.

However, I did choose two strong and lovely women as paranimphs, Sara and Maira, chuskiiii and chuliiii. I have no words to express how grateful I am for having you in my life and for the support in the last 4 years. Sara, desde el primer día que nos conocimos me ofreciste tu amistad incondicional. Eres una bellísima persona, nunca falta una sonrisa en tu boca y eso hace que estar a tu lado sea alegría asegurada. Maira, thanks for bringing sunny days to our Dutch life. Thanks for your kindness, support and friendship that have made me feel at home in Wageningen.

Pili, ¿te incluyo con la familia holandesa o la española? Muchas gracias por ser una amiga 100. Sin tu apoyo los primeros meses en Holanda, hoy no estaría aquí. Gracias por tu amabilidad y generosidad, por tus consejos, vuestras visitas con flores y merienda. ¡Eres mi luz malagueña en Holanda!

También me gustaría dar las gracias a mis amigos de España. A mis malagueñas salerosas, que aunque no nos veamos todo lo que me gustaría, sé que siempre estáis ahí para lo que haga falta. Y lo mismo se puede extender al otro extremo de la península, donde vaya tres veces al año o una parece que el tiempo no nos ha separado nunca. Bombis, gracias por el apoyo, por siempre siempre estar ahí, jos quiero! Niñas, lo hemos vuelto a conseguir! Gracias a vosotras y a los fizik@s porque aunque pasen los años seguimos encontrándonos en algún rincón del mundo como si el tiempo no hubiese pasado. Meteos, ánimo con el último empujón y gracias por estar ahí para escuchar cualquier problema, sea científico o no. Infinitas gracias a todas las visitas, vivir en el extranjero es más llevadero gracias a vosotros que me seguís allá donde vaya.

Alfonso, te estoy eternamente agradecida por tu apoyo, especialmente durante este último año. Si a alguien le tengo que agradecer estar escribiendo estas últimas líneas de la tesis, es a ti y a la gran persona que eres. Me has enseñado que todo es relativo, y que hay que darle importancia a las cosas que nos hacen felices y no dejar que las tonterías cotidianas de la vida nos influyan de forma negativa. Gracias por hacer que la distancia que nos separó durante 3 años pareciese más corta, y gracias por finalmente hacer realidad el sueño de vivir juntos bajo el mismo techo. Ahora Holanda es un sitio más agradable donde vivir solo porque tú estás aquí. Muchas gracias de corazón por quererme tanto.

Por último, si bien no menos importante: mami, papi, me faltan palabras para agradecer todo el sacrificio y apoyo incondicional que me habéis dado desde que un día decidí irme a estudiar física a Granada. Sin ese apoyo, hoy no estaría aquí. Gracias hermani por ser como eres, por reír siempre y por no faltar a la cita anual en Holanda. Al resto de la familia, muchas gracias por el apoyo y el cariño, a mis primitos por revisar el inglés y a Lourdes por darle el toque artístico a la tesis.

Yaya, ipor fin he salido en la tele! 


\section{SENSE}

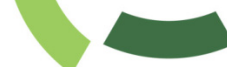

Netherlands Research School for the

Socio-Economic and Natural Sciences of the Environment

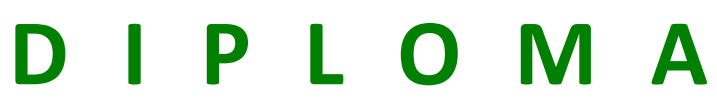

For specialised PhD training

The Netherlands Research School for the Socio-Economic and Natural Sciences of the Environment (SENSE) declares that

\section{Alba Lorente Delgado}

born on 29 March 1988 in Elorrio, Spain

has successfully fulfilled all requirements of the Educational Programme of SENSE.

Wageningen, 23 May 2019

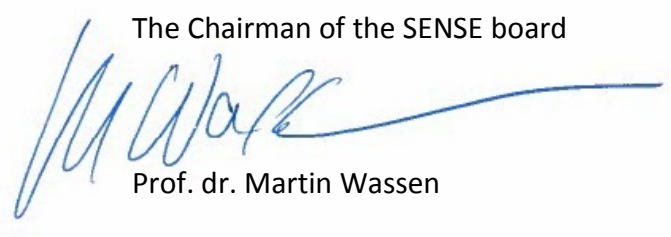

the SENSE Director of Education

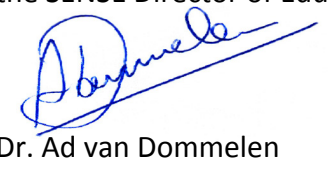

The SENSE Research School has been accredited by the Royal Netherlands Academy of Arts and Sciences (KNAW)

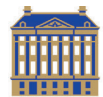

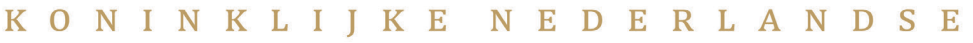

$\begin{array}{lllllllllllllllllllllllll}\text { A } & \text { K } & \text { A } & \text { D } & \text { E } & \text { M } & \text { I } & \text { E } & \text { V } & \text { A } & \text { N } & \text { W } & \text { E } & \text { T } & \text { E } & \text { N } & \text { S } & C & \text { H } & \text { A } & \text { P } & \text { P } & \text { E } & N\end{array}$ 


\section{(sase)}

The SENSE Research School declares that Alba Lorente Delgado has successfully fulfilled all requirements of the Educational PhD Programme of SENSE with a work load of $35.5 \mathrm{EC}$, including the following activities:

\section{SENSE PhD Courses}

- Environmental research in context (2015)

- Research in context activity: 'Initiating and co-organizing Wageningen edition of "Pint of Science: Research Context Activity" (www.pintofscience.nl) on diverse emerging subjects (Café Loburg, Wageningen -- 14 May 2018)'

\section{Other PhD and Advanced MSc Courses}

- Atmospheric composition and air quality, Wageningen University (2014)

- Scientific writing, Wageningen Graduate Schools (2015)

- Remote Sensing of the atmosphere, emissions and modelling, PANDA European Summer School, University of Bremen, Germany (2015)

- Presenting with impact, Wageningen Graduate Schools (2016)

- Advances in air quality analysis and prediction: the interaction of science and policy, NCAR Advanced Study Program, United States of America (2016)

\section{Management and Didactic Skills Training}

- Supervising MSc student with thesis entitled 'Quantifying NOx emissions from Paris with high-resolution satellite measurements' (2018)

- Supervising BSc student with thesis entitled 'First light for TROPOMI. A comparison between TROPOMI and OMI DOMINO v2.0 NO 2 columns' (2018)

- Teaching assistant in the MSc course 'Atmospheric composition and air quality' (20152017)

\section{Oral Presentations}

- Surface reflectance anisotropy, clouds and NO2: changing perspectives for GOME-2A and OMI. Lessons learned from QA4ECV project. 8th International Differential Optical Absorption Spectroscopy Workshop, 4-6 September 2017, Yokohama, Japan

- Monitoring nitrogen oxides emissions from build-up of pollution over Paris with S5PTROPOMI. OMI Science Team Meeting, 11-13 September 2018, De Bilt, The Netherlands - Structural uncertainty in air mass factor calculation process for $\mathrm{NO}_{2}$ and $\mathrm{HCHO}$ satellite retrievals. EOS Aura Science Team Meeting, 30 August- 1 September 2016, Rotterdam, The Netherlands

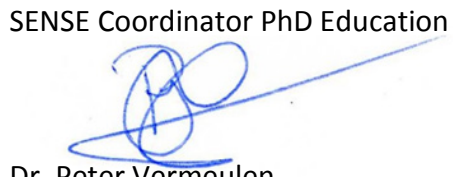

Dr. Peter Vermeulen 
This research has been supported by the FP7 Project Quality Assurance for Essential Climate Variables (QA4ECV), grant No. 607405.

Financial support from Wageningen University for printing this thesis is gratefully acknowledged.

Cover design by (C)Lourdes Garcia Soto, 2019.

Printed by proefschriften.nl 


\section{Propositions}

1. Characterization of the state of the atmosphere drives the structural uncertainty of the air mass factor.

(this thesis)

2. High spatial resolution TROPOMI NO $\mathrm{N}_{2}$ measurements can be used to estimate city scale NOx emissions on a daily basis, but only if an appropriate model guides the interpretation.

(this thesis)

3. Students of atmospheric sciences need to obtain a solid foundation in mathematical methods.

4. Carbon neutral energy consumption does not exist.

5. The fact that people do not stand up for their right to breathe clean air reflects the lack of communication between science and society.

6. Senior female mentors that encourage young women are essential to achieve gender parity in the STEM fields.

7. Migration is intrinsic to human nature, therefore it should not be managed nor treated as a problem.

8. The time zone of a region should be set based on scientific arguments and not on people's habits.

Propositions belonging to the thesis, entitled:

From photon paths to pollution plumes:

Better radiative transfer calculations to monitor $\mathrm{NO}_{x}$ emissions with $\mathrm{OMI}$ and TROPOMI

Alba Lorente Delgado

Wageningen, 23 May 2019. 


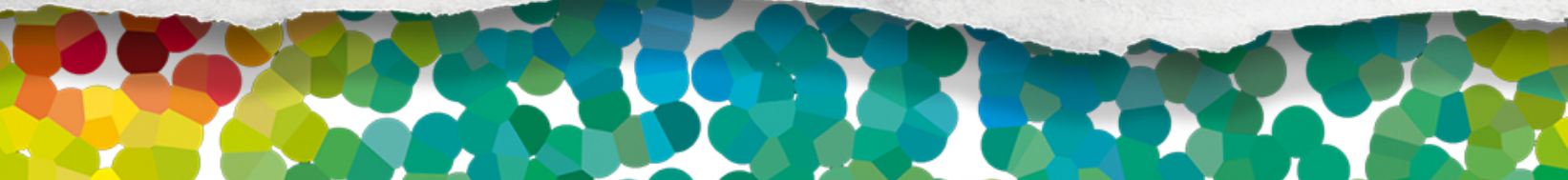
$\cot x+2+2+1$

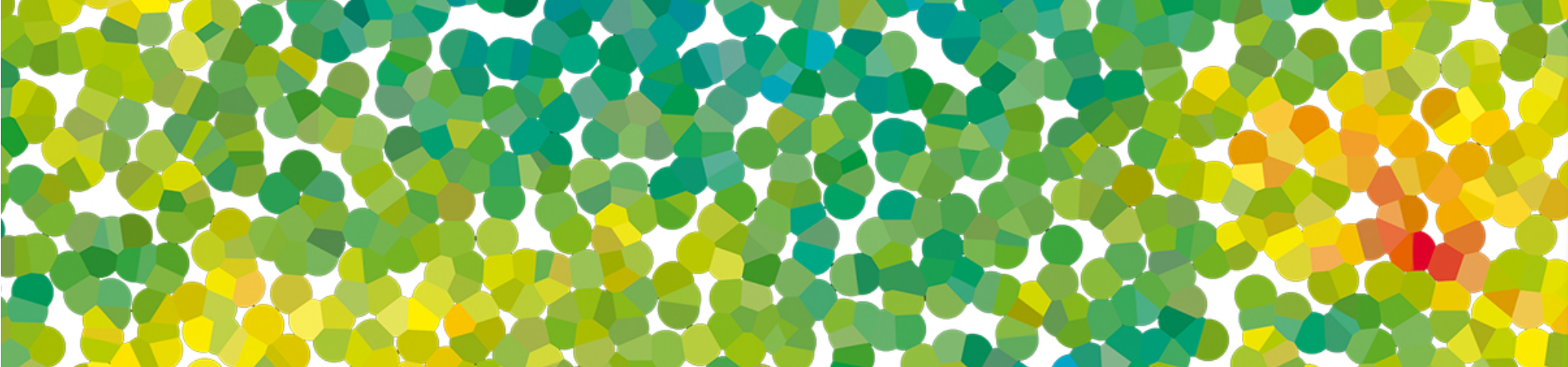
$3010+a,+1+2+1$

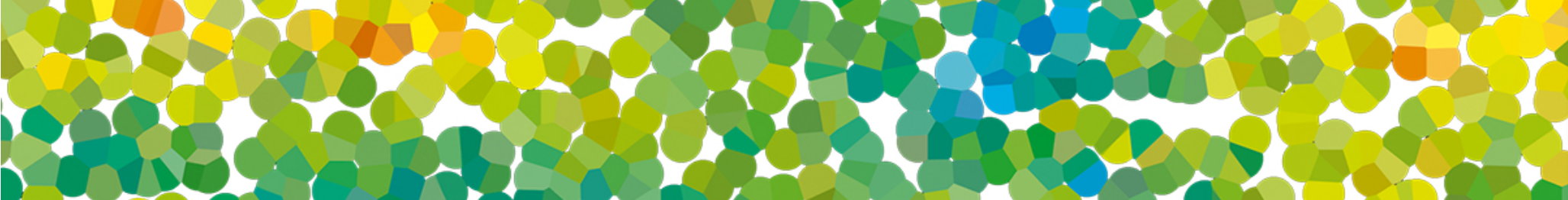

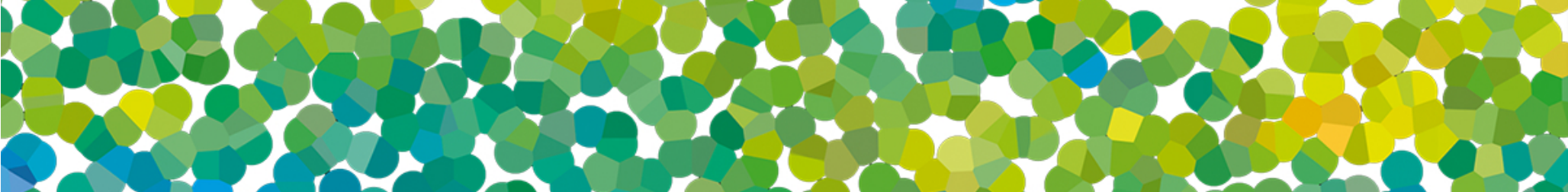

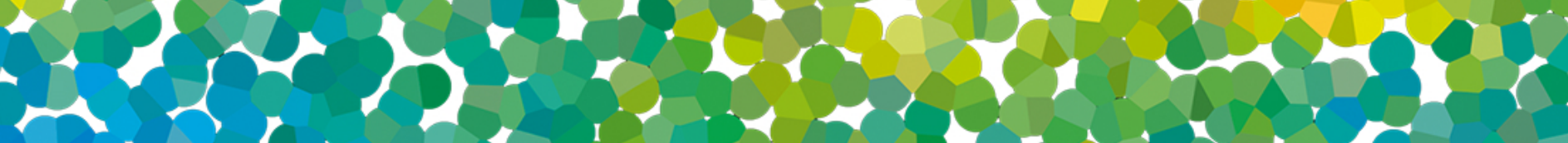

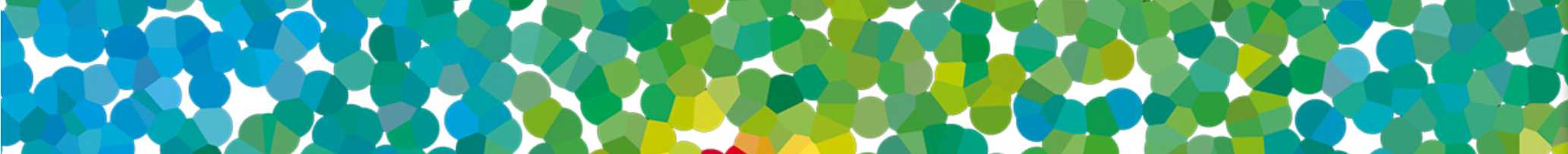

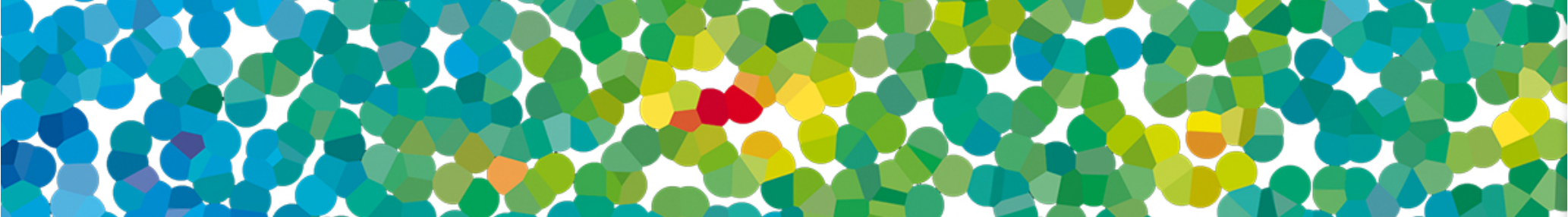

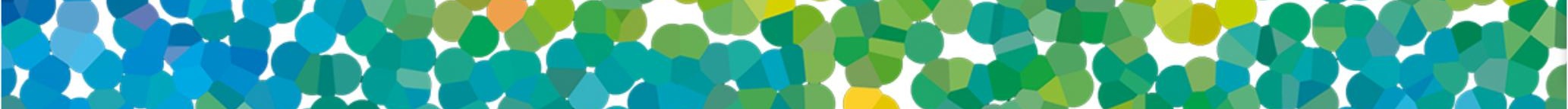

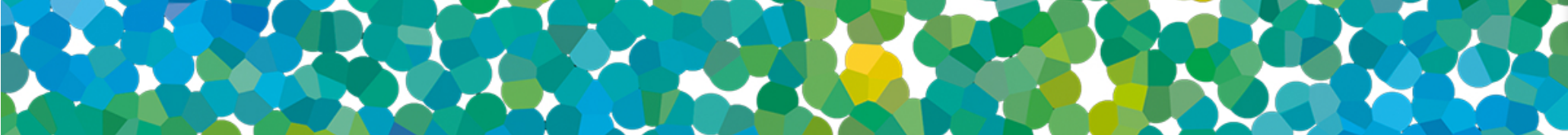

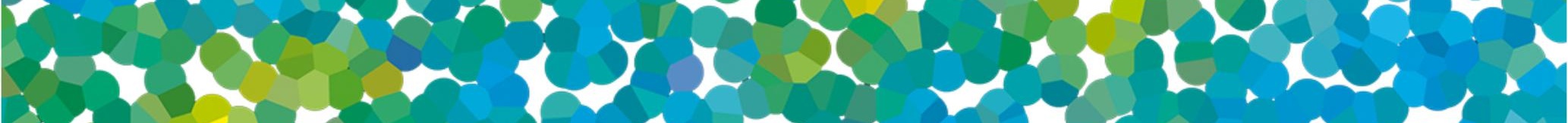

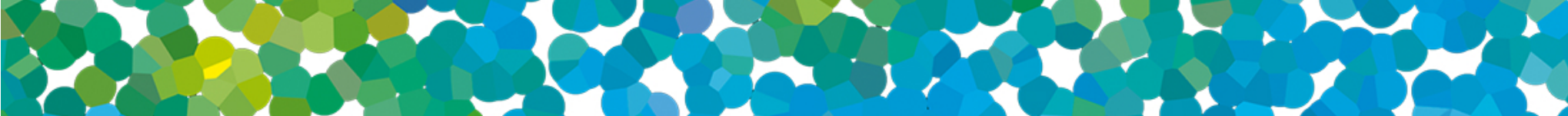

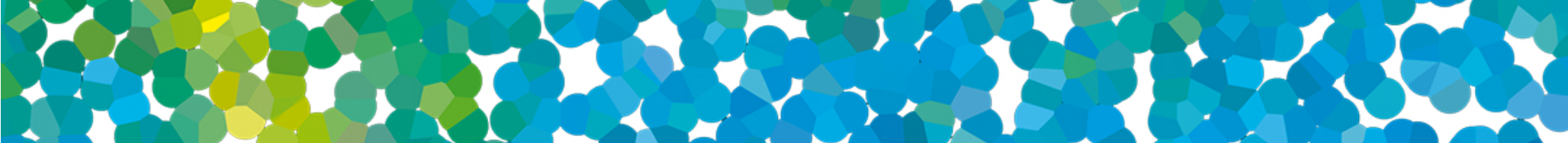
$12+2-1+2+1+1+2$

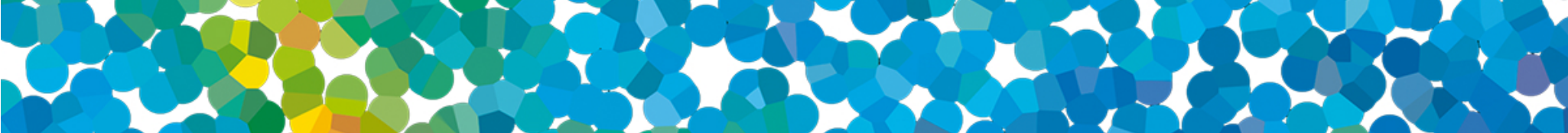

